\title{
Evaluating methane outputs from an area of submarine seeps along the northern Hikurangi Margin, New Zealand
}

\author{
A thesis \\ submitted to Victoria University of Wellington \\ in fulfilment of the requirements for the degree of \\ Master of Science in Geophysics.
}

School of Geography, Environmental and Earth Sciences Victoria University Wellington

2016 


\section{Contents}

Contents $\quad$ iii

$\begin{array}{lll}\text { Abstract } & \text { vii }\end{array}$

$\begin{array}{ll}\text { Acknowledgements } & \text { ix }\end{array}$

Glossary $\quad$ xi

Acronyms

List of Figures $\quad$ xvi

List of Tables $\quad$ xvii

1 Introduction $\quad 1$

1.1 Motivation . . . . . . . . . . . . . . . . . . 1

1.2 Greenhouse capacity of seep-emitted methane . . . . . . . . . . . . 2

1.3 Identifying evidence of seeps . . . . . . . . . . . . . . . . 3

1.3.1 Sonar profiling .................. 3

1.3.2 Environmental features . . . . . . . . . . . . . . 5

1.4 Mechanisms of gas passage to the atmosphere . . . . . . . . . . . . . 6

1.5 Seep potential of the Hikurangi Margin . . . . . . . . . . . . . . 7

1.6 Evidence of seeps along the Hikurangi Margin . . . . . . . . . . . . . 11

1.7 Recent discovery of densely populated seeps along the Hikurangi Margin 14

1.8 Objectives of this thesis f . . . . . . . . . . . . . . . 17

2 Methods $\quad 19$

2.1 Data acquisition methods . . . . . . . . . . . . . . . . 19

2.2 Water-column summed intensity maps . . . . . . . . . . . . . . . . . 21

2.3 Sonarscope . . . . . . . . . . . . . . . . . . . . . . . . 22

2.4 Matlab and Fledermaus . . . . . . . . . . . . . . . . . 25

2.5 Paridgm-GOCAD and OpendTect . . . . . . . . . . . . . 26

2.6 Bubble size and position measurements . . . . . . . . . . . . . . . . . . 29

2.7 Singlebeam acoustic sampling . . . . . . . . . . . . . . . . . . . 44 
2.8 Methane flux calculations . . . . . . . . . . . . . . . . . . 47

2.9 Error analysis . . . . . . . . . . . . . . . . . . . . . 48

2.9.1 Bubble distance from the camera . . . . . . . . . . . . . . . 48

2.9.2 Sinusoidal variations in bubble size measurements . . . . . . . . 50

2.9.3 Unquantified sources of error . . . . . . . . . . . . . . . 51

3 Results $\quad 53$

3.1 Multibeam data . . . . . . . . . . . . . . . . 53

3.1.1 Regional seep distribution and total seep count . . . . . . . . 53

3.2 Seep-bubble preservation at staggered depths . . . . . . . . . . . . 59

3.3 Bubble size and rise-rate measurements . . . . . . . . . . . . . . . 66

3.3.1 Bubble radius distribution . . . . . . . . . . . . . . 66

3.4 Singlebeam flare profiles . . . . . . . . . . . . . . . . . . 74

4 Discussion $\quad 76$

4.1 Bubble sizes . . . . . . . . . . . . . . . . . . . . 76

4.2 Characteristics of bubble ascent through the water column . . . . . . 77

4.2.1 Irregular rise paths . . . . . . . . . . . . . . 77

4.2.2 Adjustments to theoretical bubble-rise rates . . . . . . . . . . 78

4.3 Predictions of methane flux . . . . . . . . . . . . . . . . . . 82

4.4 Multiple bubble exits at the seafloor . . . . . . . . . . . . 83

4.5 Bubble survival during ascent . . . . . . . . . . . . . . . . 84

4.5.1 Relative survival rates of an assemblage of seeps . . . . . . . . 84

4.5.2 Bubble survival in a single seep plume . . . . . . . . . . . . 86

4.6 Seep distribution along the Hikurangi Margin . . . . . . . . . . . . . . 91

4.6.1 Interpreting determined seep locations . . . . . . . . . . . 91

4.6.2 Estimations of regional seafloor methane flux . . . . . . . . . . 97

4.7 Implication for global seep models . . . . . . . . . . . . . . . . . . . 97

4.8 Correlations between seep emission flux and summed-acoustic intensity 98

4.9 Future work . . . . . . . . . . . . . . . . . . . . . . . . 102

5 Conclusions $\quad 104$

6 Appendix 107

6.1 Summed_Intensity_Maps . . . . . . . . . . . . . . 107

6.2 ascii_columns ....................... 110

6.3 DTIS_Frame_Dimensions . . . . . . . . . . . . . . 110

6.4 Starting_Point . . . . . . . . . . . . . . 114

6.5 pick_image . . . . . . . . . . . . . . . . 115

6.6 Canadian_Grid . . . . . . . . . . . . . . 117

6.7 Laser_Detect . . . . . . . . . . . . . . . . . . . . 121 
6.8 Canadian_Grid_Measure . . . . . . . . . . . . . 122

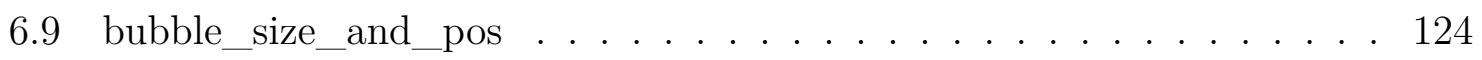

6.10 off_centre . . . . . . . . . . . . . . 127

6.11 uncertainty . . . . . . . . . . . . . . . . . . . 128

$\begin{array}{ll}\text { Bibliography } & 139\end{array}$ 


\section{Abstract}

Collated global marine surveys have documented large volumes of gaseous methane able to escape from deeply-buried deposits into global oceans as seeps. Seeps are evident where permeable faults and fracture networks allow for the upward transportation of methane from buried deposits into the water column as plumes of rising bubbles. Seep bubbles dissolve the majority of their constitutive methane into the surrounding water column as they rise; however there is evidence of more-prominent seeps transferring undissolved methane through the water column and into the atmosphere.

Due to the biologic origins of methane, the global distribution of buried methane deposits is highly varied and difficult to predict. High uncertainties in seep locations have resulted in all previous estimations of the global proportion of atmospheric methane attributed to seeps to have very large associated errors. These are mainly due to large extrapolations over global oceans based on findings from surveyed seep fields.

A 2014 NIWA research voyage saw the discovery of an abundant seep field situated at uncharacteristically shallow water depths (150-300 m below sea level) along the raised continental shelf of the Hikurangi Margin, New Zealand. In comparison to other globally documented seep fields, the Hikurangi Margin seeps are numerous (estimated between 585 and 660 surveyed seeps) and cover a large area $\left(\sim 840 \mathrm{~km}^{2}\right)$. Prior to the discovery of this seep field, there was only evidence of 36 seeps along the entire Hikurangi Margin. Acoustically surveyed bubble-rise paths of newly discovered seeps also show evidence of seeps extending the entire height of the water column. The large number of shallow flares present in the abundant seep field represent the potential for considerable amounts of gaseous methane outputs.

To further investigate these seeps, NIWA voyages TAN1505 and TAN1508 that took place in June and July of 2015 employed a range of scientific equipment to analyse features of the rising seep bubbles. Part of these investigations involved the video recordings of rising seep bubbles from the seafloor as well as acoustically surveying rising bubbles using a singlebeam and multibeam echsounder. We have used video and acoustic data sets to create multiple tools and computational techniques for better assessing features of seeps. 
We have developed photogrammetric tools that can be used in Matlab to compute bubble-size distributions and bubble-rise rates from still frames of underwater video footage. These bubble parameters have then been combined with singlebeam recorded flare profiles to calculate the flux of emitted methane at the seafloor. These calculations were carried out using the FlareFlow Matlab module, devised by Mario Veloso.

To assess the number of seeps in a multibeam surveyed region, we have created verticallysummed intensity maps of the obtained water column data. Summed-intensity maps display localised high-amplitude features, indicative of seeps. Seep indicators have been used to (1) map the distribution of seeps of the surveyed Hikurangi Margin, (2) assess the total surveyed seep count, and (3) identify regions where seep concentrations are particularly high.

We have combined methane fluxes from analysed seeps with regional seep-distribution maps to approximate the rate at which gaseous methane is escaping from the seafloor across the seep field. Extrapolating seep emissions over the surveyed area approximates $0.99 \times 10^{5} \pm 0.64 \times 10^{5} \mathrm{~m}^{3} / \mathrm{yr}$ of undissolved methane is being released across the seep field. Using models of methane preservation, combined with staggered depth models of flares, we have approximated that $\sim 0.2 \%$ of the methane emitted at the seafloor is able to reach the atmosphere. 


\section{Acknowledgements}

Firstly, a huge thank you to my supervisors Joshu Mountjoy, Gareth Crutchley and John Townend. You have all allowed me a lot of freedom throughout my project, which has given me opportunities to develop areas that I have thought interesting and important. It has been truly great to be involved in the innovative science that has gone into this thesis. Joshu, thank you for organising some great opportunities while I have been based at NIWA. From software tutoring courses to places in sea voyages, all has been much appreciated. Gareth, thank you for introducing me to the project when we were in the early stages of choosing between some possible MSc topics. I am also grateful for your input of ideas and advice on improving grammar. These are highly appreciated. John, thank you for your guiding input that has kept my work to the correct standard and time-schedule while I have been based away from the university.

I am also very grateful to the Westergaard family for the funding that I received from the Rachael Westergaard Memorial Scholarship. The financial assistance from this scholarship has allowed me to focus exclusively on my MSc research throughout the year. This project has benefited greatly from me being able to commit the time and attention that it deserves.

Other people that have contributed to the work involved in the project are: John Harper, Jean-Marie Augustin, Yoann Ladroit, Arne Pallentin and Geoffroy Lamarch. Thank you John for discussions on fluid and bubble dynamics, which were a good foundation for ideas in this thesis. Jean-Marie, thank you for teaching me more about the capabilities of SonarScope and helping improve the quality of my results that I made using the software. Yoann, thank you for adapting the EchoAnalyisis program so that flares can be modelled. Arne, thank you for answering my many questions on acoustic imaging. Geoffroy, thank you for keeping me involved in acoustic-backscatter research.

Thank you to all the people that have made me feel welcome at NIWA. I has been great being based at NIWA and I am grateful for all the access to various data, softwares, computers etc that I was able to use for my project. Special mentions go to Susi Woelz for reminding me when I needed to take a break, as well as Scott Nodder, Alan Orpin, Steve Wilcox, and Joshu for taking shifts in strictly monitoring the duration of these 
breaks. Peter Gerring and Tim Kane, it was great to share an office with both of you during the time I spent at NIWA, please keep up the high standard of work that I enforced when I was present.

Also thank you to all the people on board the TAN1505, TAN1508 and SAMSARA voyages that I was a part of. It was a great learning experience to spend that time at sea as part of so many different research objectives. Thank you also to the people on board the TAN1404 voyage, who collected the seep data that motivated this project and has been used extensively for my research.

For the time I spent at GNS, thank you to the staff for allowing my time there to be flexible and providing me with the workspace and programs that I could use for parts of my research.

To the rest of the Higgs family in Taranaki, Mum, Dad, Daisy and Rhiannon, it has been great to be able to visit and unwind in the weekends. I'll try not to make these visits a permanent fixture now that I've finally left school.

A special thank you goes to the rest of the collaborate-research group at NIWA that included: Zoe Buxton, Katie St John Glew, Rebecca McPherson, Bryn Taiapa, Sophie Wilkinson and myself. Meetings for collaborative research took place at 10.30am sharp every work day and attendance was strictly enforced (primarily by Bryn Taiapa). The extensive content of these meetings was such that follow-up sessions were sometimes required to take place at $12.30 \mathrm{pm}$ of the same day. Despite the demanding routine, it was great to spend so much time with all of you and I wish you the best of luck for all of your own research.

Also, to my flatmates over the time of the project, it's been great to hang out with such a good bunch of people between working on bubbles. Frederick "Rat" Brooks, Mathew "lad" Crooymans, Glenn "flat-jock" Eyers, Eleanor "runner-up hipster" Milne, Samuel "one-trip" Rine, Frances "Franstan" Stannard and Bryn "triple-mention" Taiapa, thanks for the great times throughout the year. I look forward to catching up with you all now that I've finished my thesis and am able to reintegrate myself into society.

Billy Mills, Tom Wilson and Andrew Johns, it has been great to spend time with you throughout the postgraduate study whether it was for work or a laugh. Tom, it was great to have you around in the crunch during pre-project part of postgraduate study. Billy, it was great to have your sound-recording wisdom when I was making a cinematic of my work. 


\section{Glossary}

Clean Bubble A bubble that is completely clean from surfactants. Clean bubble are allowed to freely oscillate as they rise.

Dirty Bubble A bubble that that is partially or completely covered in surfactant compounds. Surfactants suppress bubble oscillations, causing dirty bubbles to behave more rigidly as they rise.

Echogram An acoustically imaged 2-dimensional slice of the water column. Echograms display areas of density contrasts (e.g. gaseous bubbles in seawater) in the water column, derived from the proportion of emitted to received sonar energy.

Echosounder A hull mounted piece of equipment used for conducting acoustic surveys. By emitting successive pings and recording the time and signal strength of the returned sonar energy, echograms of the underlying water column can be constructed.

Flare Rising seep bubbles that have been acoustically imaged with an echosounder. As rising bubbles are multiply sampled by successive pings, an acoustic image is formed from the rise paths of all the bubbles that constitute a flare. Flares resultantly appear as flame-like structures in echograms.

Nadir The direction pointing directly below a particular location.

Ping Each time an echosounder emits a sound pulse and data is from the echoes.

Seep Rising fluids in the form of bubbles or dissolved gasses that are discharged from an opening in the seafloor. Seeps fluid are stored at depth below the seafloor sources and have risen through permeable fracture networks to the seafloor.

Specular Reflection Where energy (all acoustic in the case of this project) is directly reflected back off a surface. Specular Reflections result in a minimal loss of energy. 
Swath The across-track distance of the base of echograms triangles surveyed using a multibeam echosounder.

Zenith The direction pointing directly above a particular location. 


\section{Acronyms}

ASCII American Standard Code for Information Exchange. A file format that has used for storing point data.

bsl Below Sea Level. Normally referred to as a distance measured below at sea level (at 0$)$.

BSR A seismic-wave reflection from the boundary between overlying clathrate (gas hydrate) and underlying gas-saturated sediments.

CTD Conductivity, Temperature, Depth. An instrument that is lowered through the water column from a ship to take measurements of conductivity, temperature and depth.

DAS Data Acquisition System. Data collected by scientific instrumentation during sea voyages was transferred to the DAS.

DTIS Deep Towed Imaging System. An underwater camera that is lowered from a ship by a cable, equipped with cameras to record video footage and photographs.

\section{GNS GNS Science.}

NIWA The National Institute of Water and Atmospheric Research.

OFOP Ocean Floor Observation Protocol. A software on a computer connected to the camera and the ship's DAS. The software allows a user to note video times of interest.

RV Research Vessel. Niwa's main research vessel is named the RV Tangaroa.

Sv Surface Volume Strength. The mean signal strength of multiple acoustic echoes returned from a volume of contantained objects. Sv is recorded using singlebeam echosounders. 
SIS Seafloor Information System. the real time software application used on board the RV Tangaroa for multibeam data acquisition.

TAN RV Tangaroa Voyage. The prefix 'TAN' is used as a naming convention when referring to specific NIWA voyage aboard the RV Tangaroa. The suffix of all voyage names are numbered by the year and the voyage number. For example, TAN1505 is the 5th voyage of the year 2015 aboard the RV Tangaora.

TS Target Strength. A measure of the reflection coefficient of a sonar target. TS values are acquired when an object is acoustically sampled with an echosounder. 


\section{List of Figures}

1.1 Multibeam-imaged across-track curtain view of flares . . . . . . . . . 4

1.2 Hikurangi Margin Tectonic Setting . . . . . . . . . . . . . . . . . . 9

1.3 Hikurangi Margin fluid generation . . . . . . . . . . . . . . . . . . . . . 10

1.4 Seismic evidence of an uprising gas chimney . . . . . . . . . . . . . 11

1.5 Recognised seep fields along the Hikurangi Margin . . . . . . . . . . . . 12

1.6 Newly disovered seep field location . . . . . . . . . . . . . . . 16

2.1 Flare analysis workflow . . . . . . . . . . . . . . . 21

2.2 Flares imaged in echograms . . . . . . . . . . . . . . . . 22

2.3 Multibeam swath coverage . . . . . . . . . . . . . . . . . 24

2.4 Multibeam water column coverage . . . . . . . . . . . . . 24

2.5 Imaged flares and side-lobe echoes . . . . . . . . . . . . . . . 25

2.6 Bounding surface of acoustic vertical summations . . . . . . . . . . . 26

2.7 Seismic horizons as bounding surfaces for acoustic vertical summation . 27

2.8 Staggered depth model for flare analysis . . . . . . . . . . . . . . . 28

2.9 Operation sequence for computational bubble measurements . . . . . . 30

2.10 Canadian grid geometry . . . . . . . . . . . . . . . . . . . . 31

2.11 Camera field of view . . . . . . . . . . . . . . . . . . . . 32

2.12 Scaling laser detection . . . . . . . . . . . . . . . . . . 34

2.13 Constructing a Canadian Grid . . . . . . . . . . . . . . . . 35

2.14 Applied Canadian Grid example . . . . . . . . . . . . . . . . . . . 37

2.15 Geometry of bubble position relative to the camera . . . . . . . . . 38

2.16 Picking bubble axes . . . . . . . . . . . . . . . . . . . . . 40

2.17 Calculating bubble axes lengths . . . . . . . . . . . . . . . 40

2.18 Singlebeam-imaged across-track curtain view of flares . . . . . . . . . . 45

2.19 Singlebeam acoustic response of seep bubbles . . . . . . . . . . . . . . . 45

2.20 Singlebeam imaged flare profile . . . . . . . . . . . . . . . 46

2.21 Bubble deviation across the camera's view . . . . . . . . . . . . . 49

2.22 Bubble deviation towards and away from the camera . . . . . . . . . . 49

2.23 Changes in bubble-position errors with elevation differences . . . . . . . 51

2.24 Bubble orientation to the camera . . . . . . . . . . . . . . . 52

3.1 TAN1404 survey regions . . . . . . . . . . . . . . . . 55 
3.2 TAN1505 survey regions . . . . . . . . . . . . . . . 56

3.3 Summed intensity map . . . . . . . . . . . . . . . . . 57

3.4 Flare data as a seismic voxet . . . . . . . . . . . . . . . . . 60

3.5 Position and extent of summed-intensity surface calculations . . . . . . 61

3.6 Summed intensity surfaces . . . . . . . . . . . . . . . . 64

3.7 Histograms of summed intensity at staggered depths . . . . . . . . . . . 64

3.8 Cropped section summed intensity surfaces around a flare . . . . . . . . 65

3.9 Summed intensity histograms for the cropped flare . . . . . . . . . . 65

3.10 Bubble-size measurements . . . . . . . . . . . . . . . . . . . 67

3.11 Refined bubble-size measurements . . . . . . . . . . . . . . . . . . 68

3.12 Bubble-size distribution from collated measurements . . . . . . . . . . 69

3.13 Tracked-bubble size measurements over time . . . . . . . . . . . . . . . 71

3.14 Tracked-bubble elevation measurements over time . . . . . . . . . . . 72

3.15 Bubble rise rate measurements . . . . . . . . . . . . . . . . . . . 73

3.16 Singlebeam imaged flare profiles . . . . . . . . . . . . . 75

4.1 Comparisons to other published experimental bubble size measurements 77

4.2 Spline fit to tracked bubble size measurements . . . . . . . . . . . . 79

4.3 Comparisons to other published theoretical bubble rise rate predictions $\quad 80$

4.4 Photographed bubble oscillations . . . . . . . . . . . . . . . . . 80

4.5 Experimental bubble rise-rate models . . . . . . . . . . . . . . . . 81

4.6 Seep evidence from a carbonate mound . . . . . . . . . . . . . . 85

4.7 Fluid redistribution through a carbonate mound . . . . . . . . . . . 86

4.9 Bubble dissolution and pressure response models . . . . . . . . . . . . . 89

$4.104 .25 \mathrm{~mm}$ bubble dissolution model for $164 \mathrm{~m}$ water depth . . . . . . . 90

4.11 Seep distributions over the Northern Hikurangi Margin . . . . . . . . . 94

4.12 Density of seeps imaged on TAN1404 . . . . . . . . . . . . . . . 95

4.13 Density of seeps imaged on TAN1505 . . . . . . . . . . . . . . . 96

4.14 Multiple sonar lines over a single seep . . . . . . . . . . . . . . . . . 100

4.15 Flare intensity dependencies on ship speed . . . . . . . . . . . . . 101

4.16 Flare intensity association with seep-emitted methane-bubble flux . . . 101 


\section{List of Tables}

3.1 Total seep counts in survey regions . . . . . . . . . . . . . . 58

4.1 Methane fluxes emitted from seeps, based on a minimum bubble-size distribution . . . . . . . . . . . . . . . . . . . 83

4.2 Global seep field sizes and seep numbers . . . . . . . . . . . . . . 92 


\section{Introduction}

\subsection{Motivation}

Methane is a particularly potent greenhouse gas, contributing 3.7 times the global warming potential of carbon dioxide per mole in the atmosphere (Lashof and Ahuja, 1990). Methane is generated as a bi-product of many naturally occurring organic processes and is globally stored in large quantities below the seafloor. Models devised by Buffett and Archer (2004) predict that globally buried methane quantities amount to $3 \times 10^{6} \mathrm{Tg}$ of carbon stored as frozen methane clathrate and $1.8 \times 10^{6} \mathrm{Tg}$ of carbon stored as free gas. Due to its biological origin, the spatial distribution of buried methane reserves are highly varied across the Earth.

In some globally documented cases (e.g. Suess (2010); Judd and Hovland (2007); Kvenvolden et al. (2001)), buried methane has been allowed to rise to the seafloor and escape into the water column as seeps. These occur as either diffusive releases of dissolved methane or as plumes of ensuing bubbles originating from localised seafloor openings. In some locations, seeps have been observed to transport undissolved methane through the entire extent of the water column e.g. the Arctic Shelf (Shakhova et al., 2010), Coastal Southern California (Mau et al., 2007), UK Continental shelf (Judd et al., 1997) and Black Sea (Schmale et al., 2005). Studies have shown that seeps such as these are capable of emitting methane directly into the atmosphere (Shakhova et al., 2010; Mau et al., 2007).

There is potential for very large global fluxes of methane to be released into the atmosphere from submarine seeps (Clark et al., 2000). However, predictions of seepgenerated atmospheric methane are very poorly constrained as a result of the high spatial variability and poor data coverage of methane reserves that are able to escape through the seafloor. Previous approximations of the net production of global atmospheric methane attributed to seeps along continental shelves have been estimated using models of hydrocarbon reservoir depletion, migration rates and rate of removal by oxidation and measured outputs of prolific seep sites (Trotsyuk and Avilov, 1988; Lacroix, 1993; Hornafius et al., 1999; Ehhalt and Schmidt, 1978; Hovland et al., 1993). 
More recent calculations made by Judd et al. (2002) estimate a range of seep generated global atmospheric methane to be between 0.4 and $48 \mathrm{Tg} / \mathrm{yr}$. Large spatial extrapolations of localised seep occurrences have been the primary cause for uncertainty in these methods. Further difficulties arise from ephemeral time scales that flare activity varies over. For these reasons, the (most likely considerable) quantity of seep-generated atmospheric methane has not been included in previous United Nations Intergovernmental Panel on Climate Change Assessment Reports.

\subsection{Greenhouse capacity of seep-emitted methane}

Atmospheric methane has a comparatively short residence time (approximately 8-10 years (Steudler et al., 1989) in comparison to other greenhouse gasses. However, gaseous methane is able to effectively trap radiation directly by forming tropospheric ozone. These effects continue as methane is later oxidised to $\mathrm{H}_{2} \mathrm{O}$, which contributes to a stratospheric water vapour layer (Rosenlof et al., 2001). Due to these lasting radiation trapping capabilities, methane has been recorded to warm the Earth 23 times more than $\mathrm{CO}_{2}$ when averaged over 100 years (Ramaswamy et al., 2001).

In the case of seep-emitted atmospheric methane, the vast majority of gas release across the water-atmospheric boundary occurs as a result of direct transfer from rising bubbles rather than from dissolved methane (Mau et al., 2007; Clark et al., 2000). To investigate these differences, research has been carried out by Clark et al. (2000) on atmospheric emissions over the Coal Oil Points Seeps, which offers an exemplary case of ocean-atmospheric transfer of methane. Results concluded that atmospheric methane flux measurements above the seep field were approximately equal to the calculated direct emission rate expected from surfacing bursting bubbles. Conversely, transfer of dissolved methane from the ocean to the atmosphere only contributed $1.4 \%$ of the total emissions over the $280 \mathrm{~km}^{2}$ study area. Hence, most of this methane appears to be oxidised by microbes in the shallow water or mixed deeper in the water column where it is subsequently oxidised (Mau et al., 2007). Since undissolved methane bubbles comparatively transfer much larger proportions of methane to the atmosphere than dissolved methane, this thesis has focused mainly on analysing the bubbles that constitute submarine seeps.

It is possible that resultant global warming effects caused by increased atmospheric methane could then create a positive feedback loop to further increase the methaneoutput of seeps. As increasing sub-bottom temperature gradients acts to destabilise 
methane hydrates, a greater amount of free-gas will become readily available to escape through to the seafloor. The number of seeps able to contribute methane to the atmopshere then further increases as a result (Pecher et al., 2005). The prospect of a feedback loop implies that estimates of seep emissions are subject to change, and currently are likely to be increasing. Due to large uncertainties associated with current global seep-emission predictions, there is no indication of the rate at which emissions may be accelerating due to a potential feedback loop.

\subsection{Identifying evidence of seeps}

Recent advancement in water-column imaging technology have dramatically increased the rate of discovery of submarine seeps (Schneider von Deimling et al., 2007; Klaucke et al., 2010; Nakamura et al., 2015; Dupré et al., 2015). Seeps are evidence of originally buried subterranean fluids breaching the sea floor through localised seafloor openings and transmitting into the water column (Suess, 2010). Seeps are constituted by aggregations of rising bubbles and dissolved fluid. Observations of seeps (both terrestrial and submarine) have been globally noted (e.g. Suess (2010); Judd and Hovland (2007); Kvenvolden et al. (2001)); however very little is known about the timescales over which they are continually active or the fluid fluxes attributed to individual seeps.

Seeps can be identified hydroacoustically from the echoed bubble returns, geochemically by the raised concentration of ensuing gas (Faure et al., 2010) or from evidence of resultant environmental features that include chemosynthetic biological communities, development of carbonate hard grounds, pockmark depressions and mud volcanism (see Judd and Hovland, 2007, and references therein). Global observations exhibit seeps in a range of oceanographic environments spanning near-shore, continental shelf, and deep ocean (Judd et al., 2002).

\subsubsection{Sonar profiling}

Acoustic recordings from the water column, collected using sonar echosounders can be used to construct profiles of rising seep bubbles (viewed as flares, e.g. Figure 1.1). These data represent the backscattered energy of bodies in the water column, where the delay time and proportion of energy of the returned signal is indicative of the position and density contrast of each body to the surrounding sea water, respectively. In the case of areas, methane bubbles can be distinguished by their acoustic returns that 
range between approximately -70 and $25 \mathrm{~dB}$ (Figure 1.1). When surveying seeps with an echosounder, rising bubbles are multiply resampled with successive sonar pulses (termed 'pings') throughout their rise path. Recordings such as in Figure 1.1 show the rise history of the same bubbles over a time window that depends on the travel speed of the ship. Sonar echosounders used for such surveys are either multibeam or single beam echousounders.
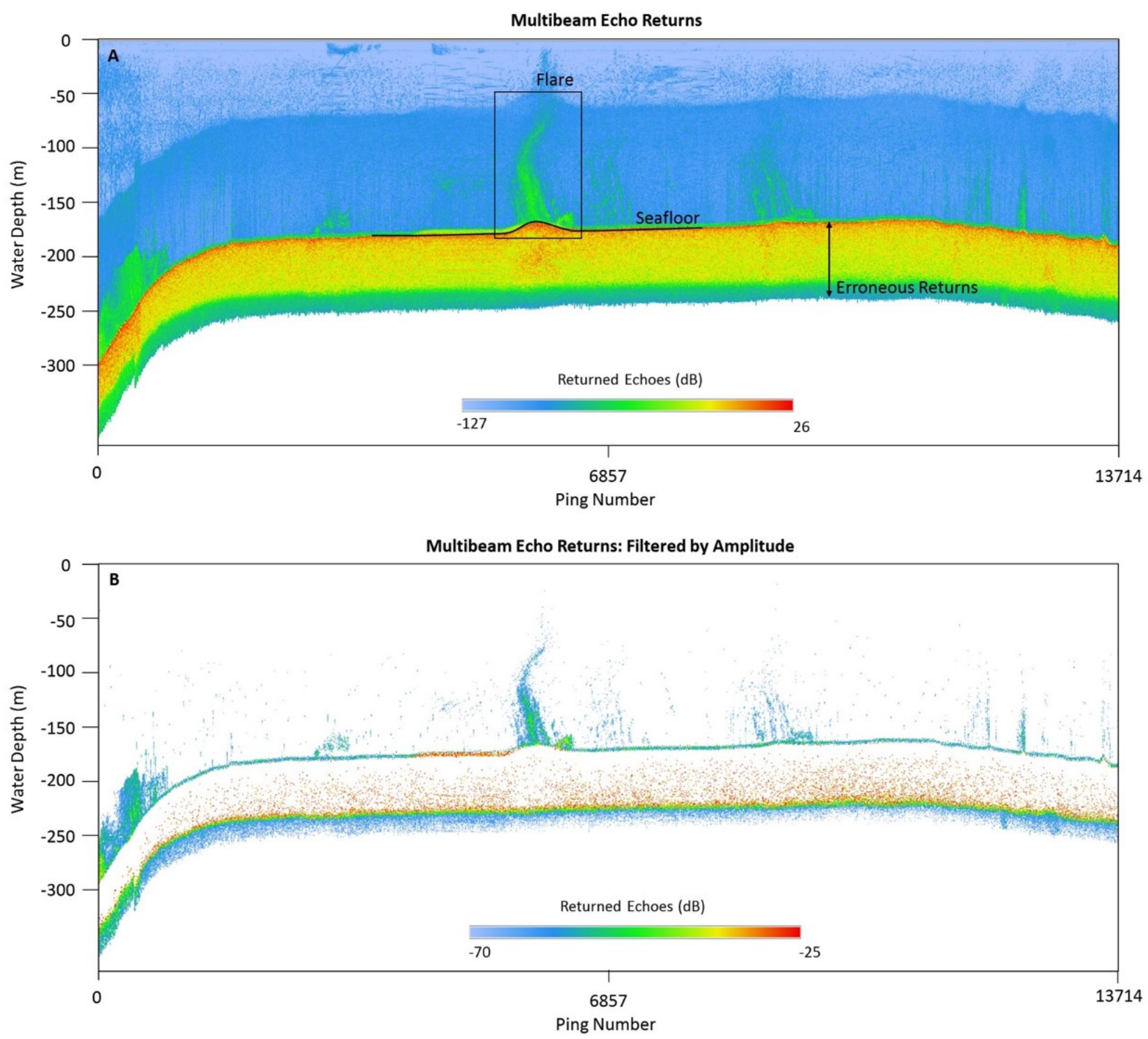

Figure 1.1: EM302 Multibeam data showing echoed returns of submarine seep bubbles located along the upper Hikurangi Margin. [A] shows data that are unfiltered by the strength acoustic returns. [B] shows the same acoustic data that have been filtered between -70 and $-25 \mathrm{~dB}$ to feature seep bubbles.

\section{Multibeam Echosounders}


Multibeam echosounders are equipped with an array of receivers angled across-track that record the echoed acoustic signal of each emitted ping. The delay times for echoes to return to each receiver is processed to produce a visual representation of an across-track triangular view of the water column (Figure 2.2). These 2-dimensional water-column representations are coloured by the amplitude of the echoed energy and termed 'echograms'. In Figure 1.1 these have been stacked horizontally to produce a curtain view of the water column. This instrumentation is very useful for surveying large areas of flares.

\section{Singlebeam Echosounders}

Singlebeam echsounders only recorded echoes using a single receiver, which produces a much smaller acoustic 'footprint' for recording water-column data. The advantage of restricting the system to a single receiver is that the returned backscattering crosssection can be used to calculate the radius of spherical objects in the water column (detailed in Section 2.7). In order to measure objects that are relatively small in comparison to the beam footprint (e.g. the radius of seep bubbles), the echosounder must be precisely calibrated. This is usually ground-truthed with a copper sphere of known radius positioned within the beam footprint of the echosounder. The returned backscatter cross-section is compensated until the returned signal strength equates to true size of the sphere. This is useful for measuring a range of bubble radii that are insonified by particular acoustic frequencies.

\subsubsection{Environmental features}

Potential seep sites can also be identified by the biological communities that survive on the fluid supply (Gibson et al., 2005). Anaerobic methane (as well as other seep fluid compositions) oxidation facilitates the formation of carbonates that sustains symbiotic bacteria, which are the nutritional foundation of these complex ecosystems. The observable megafauna of these ecosystems can consist of bivalves (mytilids, vesicomyids, lucinids and thyasirids according to Campbell et al. (2008)) and vestimentiferan tube worms, with pogonophorans, cladorhizid sponges, gastropods and sometimes shrimp (Gibson et al., 2005).

Characteristics of the faunal communities can be used to infer the productivity of fluid venting. The extent of these biological communities is proportional to the duration of past activity and whether they are living at the time of observation resolves if gas 
emissions are continuing or have since ceased. The species of fauna are also inherent of the chemical composition of the seep fluids (Bohrmann et al., 1998; Greinert et al., 2001).

Pockmarks form where seep fluids discharge through seafloor sediments rapidly enough to make them quick (i.e. liquefied), and are common where gas is present in nearseafloor sediments (Cathles et al., 2010). As new seeps form by creating subsurface chimneys, the initial upwards propagation of free gas acts like a piston to also displace water entrained in loose sediments. The flow of water causes sediments near the seafloor to become quick in the sense that grain-to-grain contact is lost and the grains are suspended dynamically by the upward flow. The quickened sediment is then removed by ocean-bottom currents, and a pockmark depression is left behind. Observed cases have varied in size and shape from 25 to $250 \mathrm{~m}$ in diameter and from 0.5 to $15 \mathrm{~m}$ in depth (Hasiotis et al., 1996; King and MacLean, 1970).

Mud volcanoes are similarly generated by the upward transfer of sediment through fluid migration. These features are structurally maintained by periodic expulsion of fluids (Guliyev et al., 1996), that build layered cones of mud breccia. These vary greatly in size and size with some up covering $100 \mathrm{~km}^{2}$ to others that span only a few tens of square metres (Dimitrov, 2002).

\subsection{Mechanisms of gas passage to the atmosphere}

For the majority of documented seeps, negligible amounts of gas are transferred via seeps to the atmosphere (McGinnis et al., 2006). Conversely, cases of surface waters with methane in excess of equilibrium with the atmosphere have been reported in shallow locations within close proximity of seeps (Judd et al., 2002). Atmospheric emissions from seeps have been documented over the Arctic Shelf (Shakhova et al., 2010), Coastal Southern California (Mau et al., 2007), UK Continental shelf (Judd et al., 1997) and Black Sea (Schmale et al., 2005).

In addition to seeps being situated at shallow depths, the probability that seep bubbles will survive the ascent though the water column is dependent upon bubble size, bubble rise velocity, the presence of surfactants on the bubble surface (Judd et al., 1997; Leifer and Patro, 2002) and the number of proximal bubbles and ambient methane levels. As bubbles rise, the contained gaseous methane remains in contact with seawater and rapidly outflows, resulting in bubble dissolution. This removal causes a decrease 
in bubble size far more rapidly than the expansion that results from a decrease in pressure at shallower water depths (Rehder et al., 2009). Bubble-size reduction then subsequently cubically decreases the rate of gas that is dissociated from the bubble (MacDonald, 2002).

As for the presence of surfactants, increasing the concentration will effectively reduce the permeability across the liquid-gas interface so that diffusion is slowed (Hanwright et al., 2005). The uneven distribution of surfactants around the bubbles surface will also drastically decrease the bubbles rise velocity, a phenomenon known as the Marangoni effect. Decreased rise rates occur as a result of tangential shear stresses due to an increasing variation in surface tensions that opposes the rising forces of bubbles (Takagi and Matsumoto, 2011). However, the net result is still for the bubble to have increased preservation through higher elevations with a greater concentration of surfactants (Fdhila and Duineveld, 1996). As the diffusion rate is proportional to the square-root of water-exposure time (Duda and Vrentas, 1969), increased bubble velocity (proportional to the physical water conditions, gas properties and number of rising bubbles) will increase the probability that seep bubbles will reach the surface. Bubbles rising en masse will create upwelling fluid flows that aid the advecting movement of all bubbles contained within close proximity. Finally, the ambient levels of $\mathrm{CH}_{4}$ in the water will influence the dissolution gradient across the liquid-gas interface. Rehder et al. (2009) show that higher concentrations of dissolved methane will prolong the life of proximal bubbles.

\subsection{Seep potential of the Hikurangi Margin}

It is to be expected from the structural composition of the Hikurangi Margin, that the margin accommodates a laterally continuous population of seeps. The $25 \mathrm{Myr}$ old convergent plate boundary (Barnes et al., 2010) is situtated at the southern end of the Tonga-Kermadec-Hikurangi subduction zone and extends south until it intersects the terrestrial trace of the Alpine Fault. The active margin has formed in response to the westward subduction of the bathymetrically elevated oceanic Hikurangi Plateau (Pacific Plate) beneath the overriding Australian Plate. The nearest 100km of Pacific Plate that approaches the boundary dips gently at an angle of $3^{\circ}$ to horizontal (Barnes et al., 2010) before steepening and subducting (Davey et al., 1986b; Henrys et al., 2006; Barker et al., 2009) beneath the North Island at rate of 40-50 mm/yr.

The margin exhibits evidence of spatially varied evolution and activity (Lewis and 
Pettinga, 1993; Collot et al., 1996; Field and Uruski, 1997; Barnes et al., 2002). For the central part of the margin where seeps have been previously documented (Figure 1.5), there exists an imbricated thrust wedge dominated by accretion (Davey et al., 1986a; Lewis and Pettinga, 1993; Collot et al., 1996). The wedge consists of eroded Plio-Pleistocene trench sediments from the Hikurangi Trough (Barnes et al., 2010). These sediments form margin-parallel ridges and basins on the lower and upper slope. The higher elevated subdivision of the margin (Figure 1.2) is alternatively formed from a deforming backstop of late Cretaceous and Palaeogenic passive margin sediments that predate subduction (Barnes et al., 2010).

The origin of vent fluid generation and transportation is attributed to the dewatering of the lower subducting plateau (Figure 1.3). Fluids contained in saturated trench sediments entering the accretionary wedge are expelled from the Pacifc Plate in the changing stress regime (e.g. Davis et al. (1983). A first order estimate of $24 \mathrm{~m}^{3}$ of fluid per meter of strike length per year is injected into the margin from the Pacific Plate (Townend, 1997). More than $80 \%$ of this has been attributed to compaction, with an additional $3 \mathrm{~m}^{3}$ per metre of strike length per annum released by smectite dehydration. Proximal to the dehydrating plate, hydrocarbon generation may occur from organic matter (Figure 1.3) subject to high compressional stress and rapid burial and heating (Bangs et al., 1990; Vrolijk, 1990).

Clear spatial relationships between seeps and major seaward-vergent thrust faults suggest that transportation of fluids from deeper sources to the near surface is likely via permeable thrust faults and fractures (Barnes et al., 2010). Buoyant free gas that exists below imaged bottom simulating reflections (BSRs) is able to migrate through conduits of these sort in cases where impermeable hydrate layers are structurally compromised in tectonic displacement events. Conduits are likely to accommodate the majority of free gas movement in the system as fracture networks offer a path of least resistance over less-permeable stratigraphic pathways (Tryon et al., 2002).

Seep observations in combination with structural knowledge of the margin suggest deep fluid is transported towards the outer (western) edge of the deforming Cretaceous and Paleogene inner foundation rocks. Here in the deforming backstop, methane clathrates are unstable due to the disruption caused by faults that progressively steepen further westwards. As faults reach higher gradients, they are more likely dilated (Lewis and Marshall, 1996), allowing larger fluxes of fluid to be more steadily transported upwards through them (Lewis and Marshall, 1996).

Faulting and fracturing similarly aid the redistribution of fluid reserves stored at near -surface depths, which have in some cases migrated to the surface and expelled as 


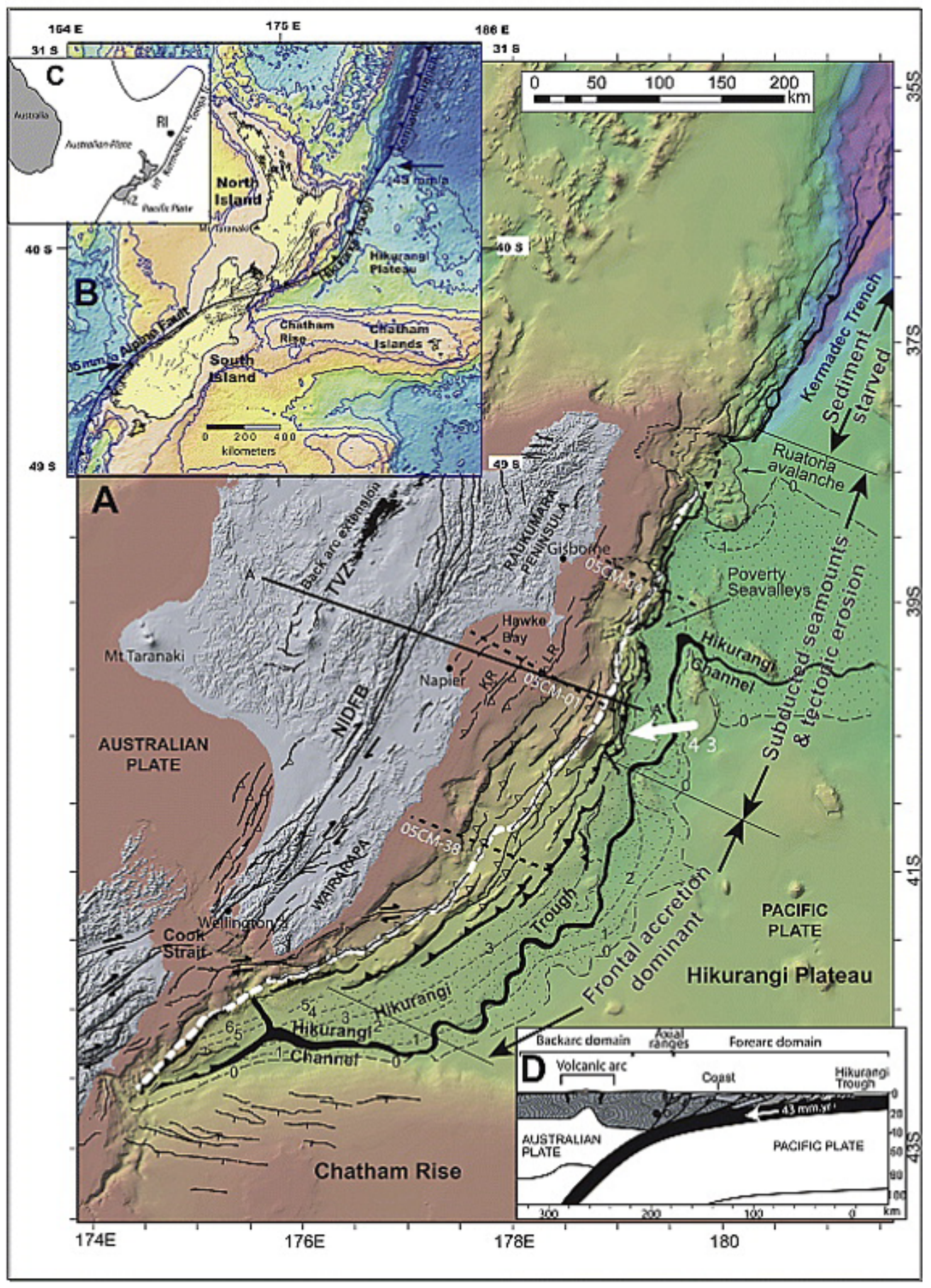

Figure 1.2: Tectonic setting of the Hikurangi Margin. Modified from Barnes et al. (2010) in Wallace et al. (2009). [A] Bathymetry (NIWA) and topography, where active faults are also displayed as black lines. The bold white dashed line illustrates the boundary between the accretionary wedge and the deforming buttress of Cretaceous and Paleogene rocks covered by Miocene to recent slope basins. The line A-A' denotes the cross-section location in $[\mathrm{D}]$. [D] illustrates the relative motions between the Australian plate and subducting Pacific Plate for the region. $[\mathrm{B}]$ and $[\mathrm{C}]$ display successively further extended views of the regional tectonic setting. 


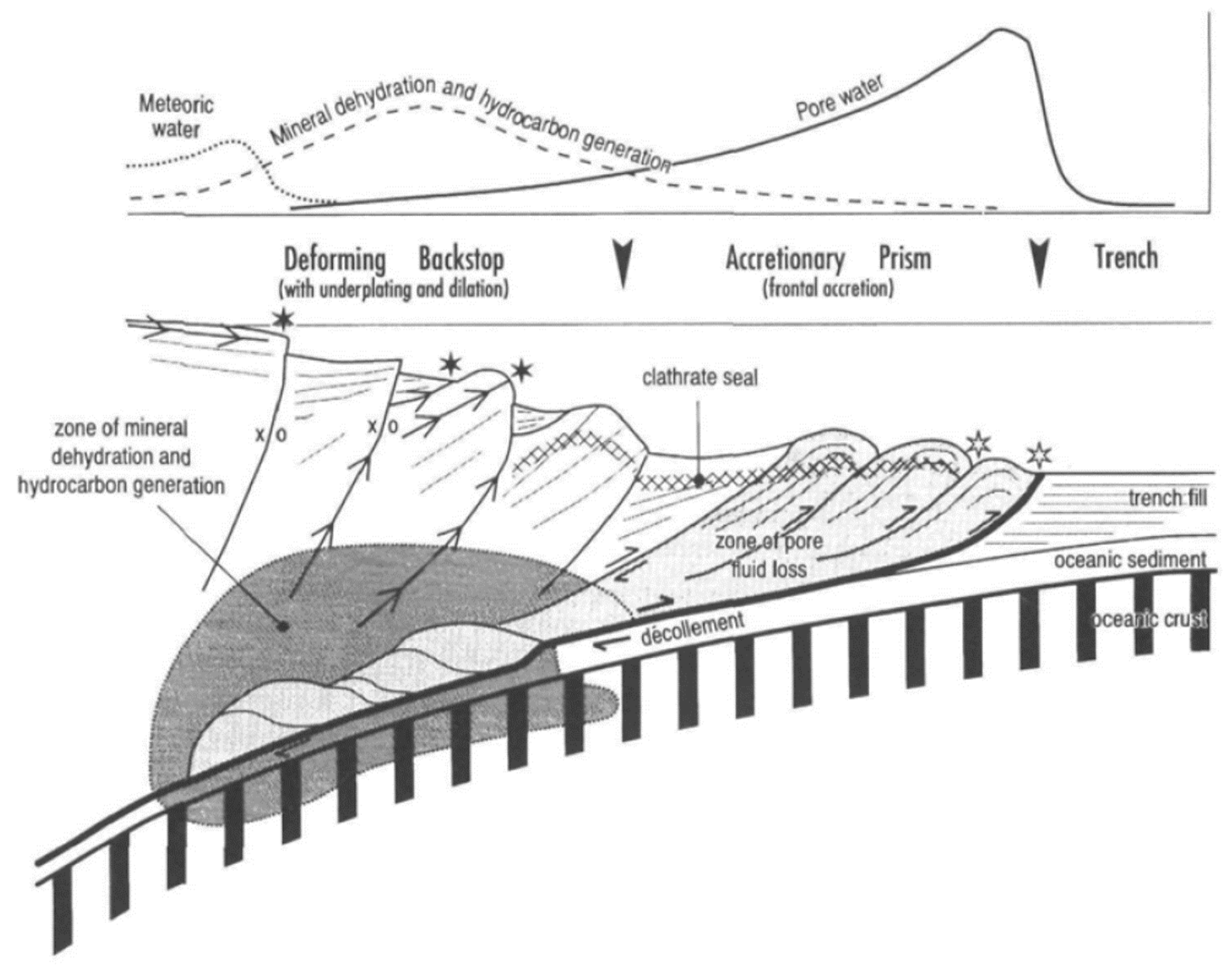

Figure 1.3: Diagrammatic cross section of the Hikurangi Margin from Lewis and Marshall (1996) illustrating the generation of fluids (based on Moore and Vrolijk (1992)). Pore water is lost from the subducting Pacific Plate, which may escape through permeable seafloor breaches (stars) or contained below an impermeable seal of methane of methane clathrates (marked by asterisks). Fluid escapes (arrows) occur at higher elevations when this seal is breached by faulting. 
seeps. Seismic profiles (conducted on the RV Tangaroa) recorded over active seep sites (Figure 1.4) display evidence that lighter hydrocarbon gasses are supplied to seafloor vents via near vertical pathways of advecting gas (Crutchley et al., 2010). These are identified as subterranean chimneys of high reflectivity where the soft sediment has been fractured by over-pressured gas (Lee and Collett, 2001).

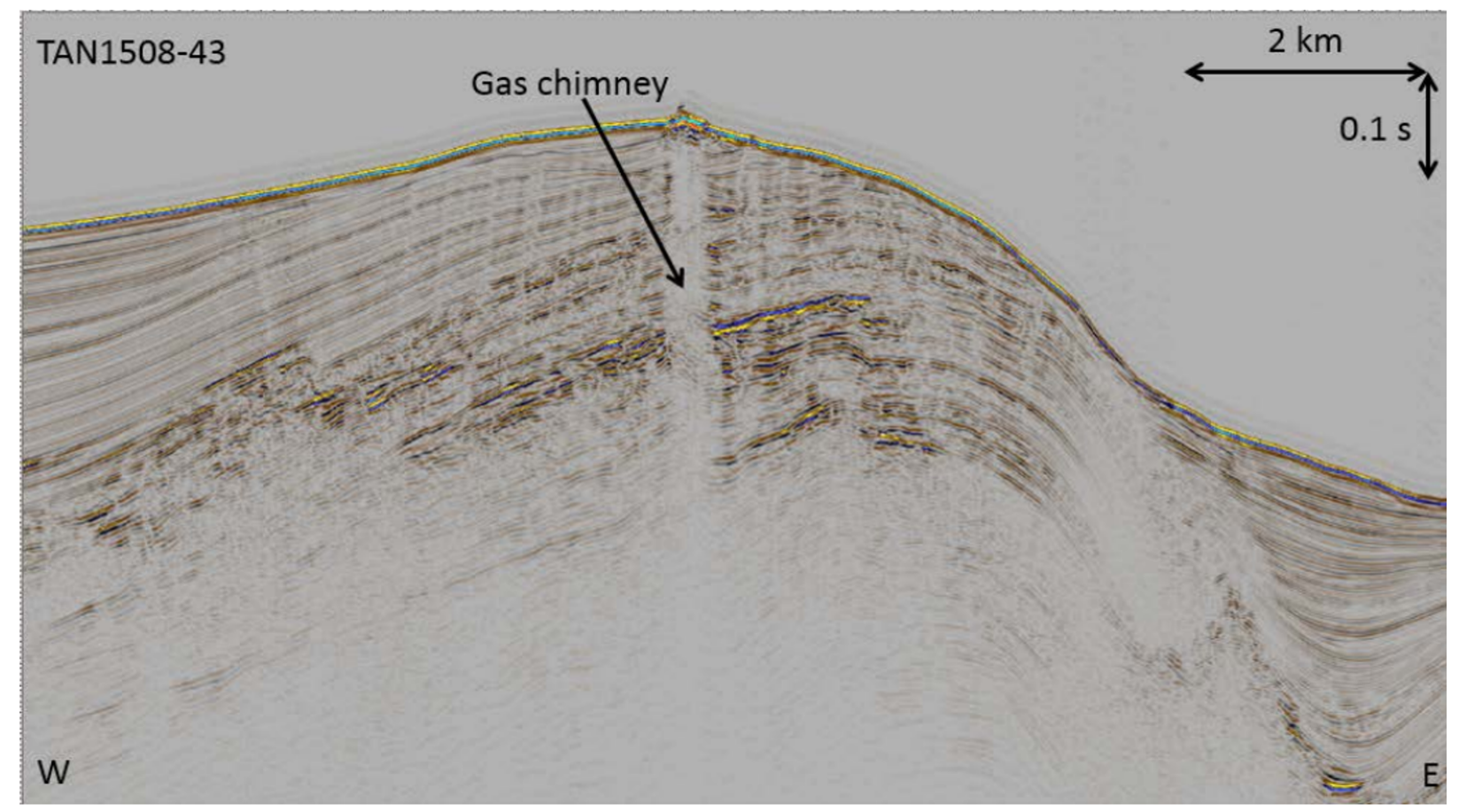

Figure 1.4: Seismic profile of a subterranean gas chimney in the centre of Uruti Ridge from Mountjoy (2015b). Gas-altered sediment can be seen as a blanked zone that extends upwards through stratigraphic layers.

The formation and maintenance of permeable fluid pathways are a primary control on the ephemeral time scales that seep intensity appears to vary over. As the permeability of rocks and fault zones is at least one-million times more variable than fluid pressures in convergent margins (Lewis and Marshall, 1996), these times scales are extremely difficult to predict.

\subsection{Evidence of seeps along the Hikurangi Margin}

The Hikurangi Subduction margin has been the focus of many research developments involving hydrocarbon seeps (Greinert et al., 2010; Lewis and Marshall, 1996) and have resolved distinct areas of concentrated seepage. These sites have been named: Rock Garden, Ritchie Ridge, Omakere Ridge, Uruti Ridde and Opouawe Bank (Figure 


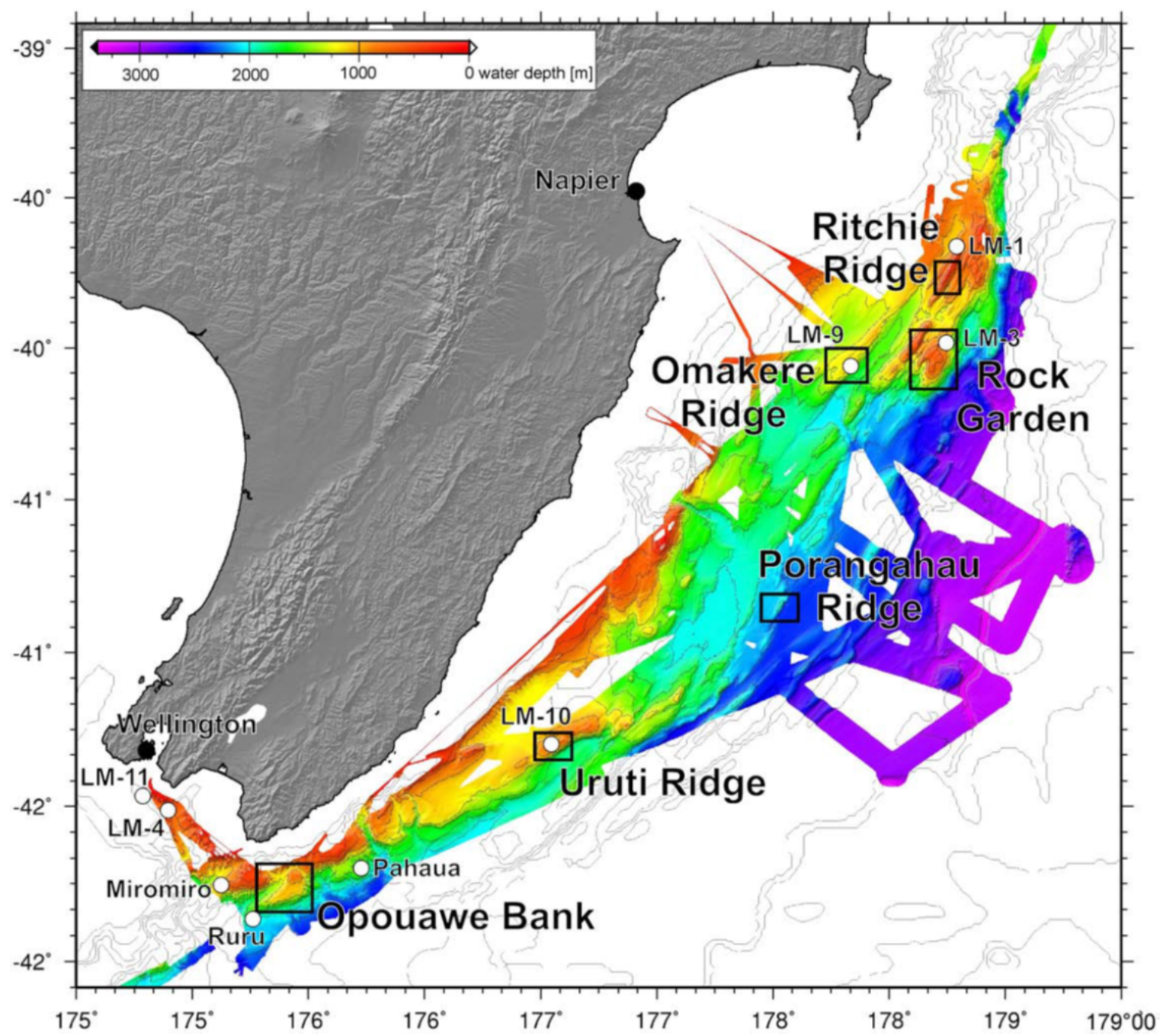

Figure 1.5: Multibeam bathymetry data from parts of the Hikurangi Margin, collected during TAN0607, TAN0616 and SO191. Distinct fields of documented seep activity have been outlined with black boxes. More localised seepage areas have additionally been labelled with white dots (Greinert et al., 2010; Lewis and Marshall, 1996). 
1.5). All of these seepage locations exist along the densely thrusted accretionary wedge (Barnes et al., 2010).

Seeps along the margin align with seaward vergent thrust faults surfacing within a depth window of 700-1200 m below sea level (bsl) (Greinert et al., 2010). The vertical extent of these seeps can be assessed from their acoustic profiles acquired from singlebeam and multibeam water-column data. Previous recordings have shown that the concentration of constituting bubbles gradually decreases upwards from the seafloor, with detectable traces still recorded up to approximately $300 \mathrm{~m}$ above sea floor (Greinert et al., 2010).

Widely varying sizes of chemotropic ecosystem indicate varied past seep activity. Some sites exhibited densely compacted carbonate cementation, where a few had formed 50 $\mathrm{m}$ high knoll structures (Greinert et al., 2010). The presence of carbonate mounds indicates seep activity over thousands of years (Berndt et al., 2014; Liebetrau et al., 2010). Other seep sites record observations of very large areas paved with clam shells and tube worms. Thriving areas such as these indicate strong and long lasting activity prior to these voyages (Greinert et al., 2010). Other sites displayed similar biological signatures without active seepage, suggesting a dormancy period over an ephemerally active timescale, analogous to other globally active seeps. In other cases, similarly large areas of fauna were found to be deceased, indicating that sites had become extinct completely (Greinert et al., 2010).

The observed species of fauna that inhabit these chemoherm complexes are recorded to grow in response to methane, with a select few sites showing evidence of fauna that grow in response to sulphide (Bohrmann et al., 1998; Greinert et al., 2001). The presence of methane is also confirmed by sightings of fist-sized chunks of solid gas hydrate in dredges from the TAN0616 voyage and (Greinert et al., 2010) and isotopic anaylsis of seep gas composition (providing a methane/ethane ratio of 48) and a proximally located core gas compostion (providing a methane/ethane ratio of 12000) (Kvenvolden and Pettinga, 1989).

Acoustic profiles display the backscattered energy of bodies in the water column (see Figure 1.1). The position and density contrasts to the surrounding water of these bodies are obtained from the delay time and proportion of energy of the returned acoustic signal, respectively. In the case of seeps, methane bubbles can be distinguished by an acoustic return that ranges between $\sim-70$ and $-26 \mathrm{~dB}$. Bubbles were repeatedly sampled by successive pings throughout their rise path. Recordings such as in Figure 1.1 show the rise history of the same bubbles over time, which produces a flare shaped image of the seeps. 


\subsection{Recent discovery of densely populated seeps along the Hikurangi Margin}

The TAN1404 research voyage undertaken by NIWA in July 2014 discovered a concentrated area of uncharacteristically shallow (150-300 m bsl) seeps situated on the raised continental slope of the Northern Hikurangi Margin (Figure 1.6). These are situated at elevations much higher than seeps that have been previously documented along the margin (Greinert et al. (2010), also see Figure 1.6). Acoustic profiles show evidence that these seeps are producing bubbles capable of rising the entire vertical extent of the water column (e.g. Figure 1.1).

Two subsequent voyages, TAN1505 and TAN1508 then took place in June and July of 2015 to gain further coverage of the seeps with a range of specialised water column analysis instrumentation. I was able to participate in these voyages and assist in collecting a wide coverage of data over the prospective seep sites. Surveys that took place recorded evidence of seeps, numbering in the order of a few hundred, situated within $\mathrm{a} \sim 50 \mathrm{~km}^{2}$ area. Acoustic profiles confirm that a large number of these are seeps that similarly extend the height of the water column.

Similar to the rest of the margin, fauna indicative of methane production were observed in underwater camera footage. These fauna are varied, with a few cases of small ( $\sim 50 \mathrm{~cm}$ radius) carbonate precipitate deposits.

The large number of seeps that appear to reach sea level suggest that a considerable quantity of methane is being released into the atmosphere. Since these acoustic observations are made over a short instance in comparison to the ephemeral timescales that seep intensity varies over. Presumably these seeps are intermittently releasing methane into the atmosphere. Atmospheric emissions are likely to occur during high flux intervals at the base of the seep, which prolongs bubble survival, increasing the chance of undissolved methane reaching the surface.

Until the finding of these shallow seeps it had been believed that there were no atmospheric gas emissions that could be attributed to seeps along the Hikurangi Margin. The rising bubbles of the deeper, previously documented seeps are completely lost due to either dissolution in the surrounding water (following parameters stated Section 1.4) or consumption by proximal chemotrophic organisms (Allison et al., 2008). 
New observations of the concentrated region of seeps indicate that the fluid output of Hikurangi Margin seep field may be comparable to other globally renowned seep fields (Suess, 2010; Judd and Hovland, 2007; Kvenvolden et al., 2001). As only a few seep field of this capacity have been discovered, new information gathered from this seep fields may be useful in improving global seep model predictions. 


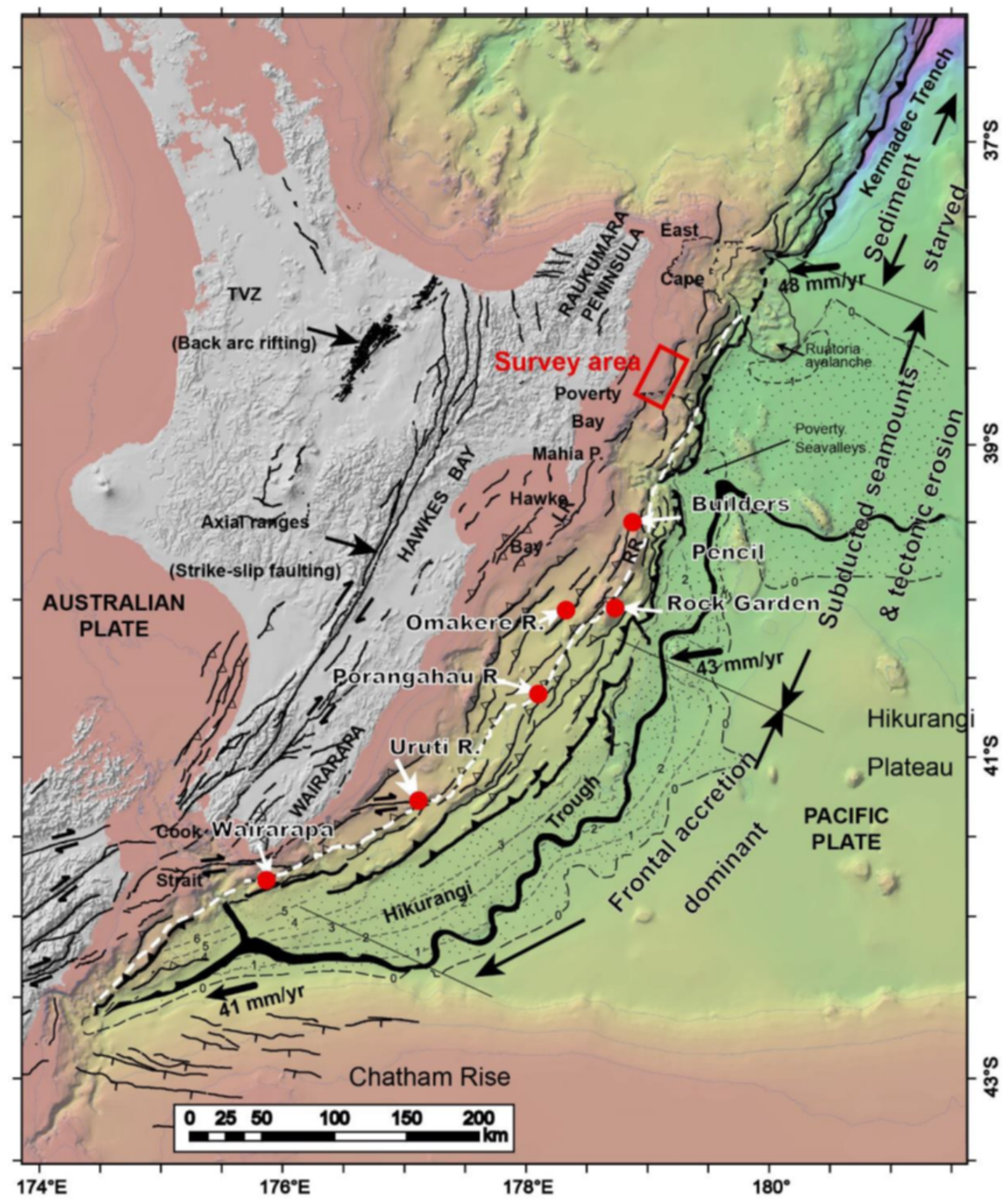

Figure 1.6: Map of previously discovered seep field sites (red dots) as well as a newly discovered area of shallow seeps from Mountjoy (2015a) 


\subsection{Objectives of this thesis}

In order to better constrain the large uncertainty associated with seep output measurements, this thesis focuses on developing improved computational techniques for evaluating seep features. We have focused these techniques towards mapping seep locations, assessing the preservation of undissolved seep fluids and quantifying the initial flux of ensuing seep bubbles. We have then applied these techniques to the recently discovered densely-populated seep field along the upper rise of the Northern Hikurangi Margin (see Section 1.7). This seep field was discovered in April 2014 and resurveyed in July 2015 to provide excellent data coverage of seeps from a range of instrumentation. By combining newly developed techniques with the data set, we will be able to make approximations as to the regional seep outputs along the Hikurangi Margin. These results will contribute to the understanding of global seep fields and their outputs. Whilst developing and applying these techniques (explained herein), we have aimed to make them generally applicable, so that they can be reapplied to other global seeps.

\section{Produce regional seep-field maps}

The spatial distribution and total seep count of a seep field will be evaluated using summed-echo intensity maps. We will map the locations of all flares within a survey region and assess the scale of fluid release across the seep field. We will also use this information to locate regions where flares are most concentrated, which can be used as an indicator for seeps that are likely transporting methane to the atmosphere (for reasons detailed in Section 1.4).

\section{Evaluate localised flare depletion}

We will then apply similar echo-summation techniques to a single seep in order to assess the preservation of seep bubbles at shallower water depths. The preservation of methane in rising seep bubbles is poorly understood as bubbles are produced ephemerally and rise chaotically. We will make first order approximations by combining acoustically imaged rising bubbles with models of methane dissolution. These approximations will be minimum estimates of the proportion of seafloor methane output that is potentially reaching the atmosphere.

\section{Quantify methane flux from submarine seeps}

A recently released fluid flow-rate calculator (Veloso et al., 2015) will be applied to acoustically image seeps in order to calculate the flux of undissolved methane that is emitted at the seafloor. The calculator resolves gaseous seep discharge in $\mathrm{mL} / \mathrm{min}$ based on singlebeam flare profiles, bubbles-size distributions and bubble-rise rates. 
To refine the flux estimates from the calculator we have developed photogrammetric tools for estimating the size and rise velocity of bubbles. These can be applied to underwater video footage at the base of the seep to measure bubble parameters in the camera's field of view. The photogrammetric techniques are accurate in comparison to previous methods of measuring bubble dimensions, which allow us to compare observations to predicted bubble behaviour in theoretical models. 


\section{Methods}

\subsection{Data acquisition methods}

Flares were acoustically imaged in the water column during three research voyages aboard RV Tangaroa using an EM302 Multibeam Echosounder System and an EK60 Singlebeam echosounder. The first of the three voyages (TAN1404) took place in July 2014 and saw the opportunistic discovery of a dense field of seeps in shallow $(<250$ $\mathrm{m}$ depth) water. Other, more sparsely distributed flares were also imaged using the EM302 system whilst conducting regional studies in the area during this voyage.

The TAN1505 voyage was then conducted in June 2015 with the specific purpose of collecting data on the physical and chemical features of a representative proportion of the seeps. EM302 Multibeam and EK60 Singlebeam acoustic datasets were collected concurrently over areas of seepage that had been identified during the TAN1404 voyage. Localized zones of prominent seepage that were discovered during acoustic data acquisition were then resurveyed using a conductivity, temperature, depth (CTD) profiler and a Deep Towed Imaging System (DTIS).

More EM302 multibeam data were collected over the region one month later during the latest of the three voyages, 'TAN1508'.

\section{EM302 Multibeam Data}

Multibeam data was collected over seeps using a Kongsberg Simrad EM302 multibeam echosounder, hull mounted on the RV Tangaroa. This operates at a frequency of $30 \mathrm{kHz}$ with an angular sector of $140^{\circ}$, across track coverage of 3-5 times water depth (depending on depth and mode) and a maximum ping rate of $10 \mathrm{~Hz}$. The system applies beam focusing to both transmited and received beams in order to obtain maximum resolution. Dynamic focusing was applied to all received beams. The transmitted beams are electronically stabilised for roll, pitch and yaw, while the received beams are stabilised for roll movements. Seafloor Information System (SIS) was the real time software application used on board the vessel for multibeam data acquisition. 


\section{EK60 Singlebeam Data}

Split-beam data were acquired using five Simrad EK60s echosounders operating at 18, 38, 70, 120 and $200 \mathrm{kHz}$, with a beam-width of 12 degrees for the $18 \mathrm{kHz}$ and 7 degrees for the others. Flare data are selected and saved using a Matlab programme 'Echo Analysis'. This is conventionally used for fisheries research at NIWA and has been modified by Yoann Ladroit to accommodate for viewing and saving acoustic flare profiles.

\section{CTD Profiles}

The presence and concentration of dissolved methane was analysed using vertical chemical profiles, sampled by a CTD on its rise through the water column. These profiles were collected at seven selected seep sites and three other stations within the survey area.

Samples were collected in $250 \mathrm{ml}$ serum bottles from the CTD Niskin bottles and analysed using the nitrogen headspace technique with flame ionization detection (FIDGC). At selected depths, samples were also collected for methane 13C-isotope analysis at NIWA's Greta Point office, and for alkalinity and dissolved inorganic carbon (DIC) analysis at NIWA's Otago office.

\section{DTIS Camera Footage}

The DTIS is towed approximately $2 \mathrm{~m}$ above the sea floor at 0.6 knots and is attached to the ship from a standard CTD cable. The system uses a Canon 10 megapixel digital SLR camera with a $24 \mathrm{~mm}$ lens to capture still images every $15 \mathrm{~s}$, and a Sony 1080P camcorder to capture high-definition colour video. Both the still camera and camcorder have a pair of lasers attached $200 \mathrm{~mm}$ apart to enable scaling. During each DTIS transect, observations of seafloor organisms and habitat were recorded using Ocean Floor Observation Protocol (OFOP) software on a computer connected to the camera vehicle and the ship's data acquisition system (DAS). After each transect, the camera was brought back on board and data were downloaded. 


\section{FLARE LOCATION/ANALYSIS WORKFLOW}

REGIONAL-SCALE MAPPING

- Produce regional maps of seep fields

- Locate trends and concentrated areas of flares

- Pick flares of interest based on: shallow sites, seafloor features, high relative flare intensities
LINE-SCALE MAPPING

- Compare flares over specified depth windows

- Analyse particular parts of flares to see how the intensity is preserved with increasing elevation
SONARSCOPE

MATLAB/FLEDERMAUS

\section{PARIDIGM-SKUA/OPENDTECT}

- Study detailed features of an individual flare

- Quantitatively analyse the changes in summed intensity across different parts of the flare

Figure 2.1: Workflow displaying the ordering of computational techniques for analysing flares using summed intensity maps.

\subsection{Water-column summed intensity maps}

We used multibeam backscatter data to locate and analyse the reflective characteristics of submarine gas seeps. To do this efficiently, we have designed a workflow that employs tools from multiple software packages (outlined in Figure 2.1) to generate summed intensity maps for the water column using different vertical integration methods. These effectively highlight regions of higher acoustic impedance (i.e. seep bubble plumes) in contrast with the surrounding water. This information is useful for comparing relative backscatter intensities between seeps, assessing bubble preservation in the water column and identifying the most prominent seeps.

To compute these maps, each program uses the amplitude values of reflective points in the water column that are present in multibeam echograms (Figure 2.2). These points are initially filtered by amplitude, so that only reflectors with a sea-water density contrast in the range of undissolved methane remain for calculations. The remaining points are likely indicative of methane bubbles, buoyancy bladders of fish (Barr and Coombs, 2005; Urick, 1967) (which will both be termed 'gaseous bodies' in this thesis) and echoes from the seafloor.

In order to isolate data that are indicative of gaseous bodies in the water column, the sea-floor echoes must be discarded prior to the integration calculations. Arcs of high-amplitude reflectivity are generated from sound fronts echoing off relief features 

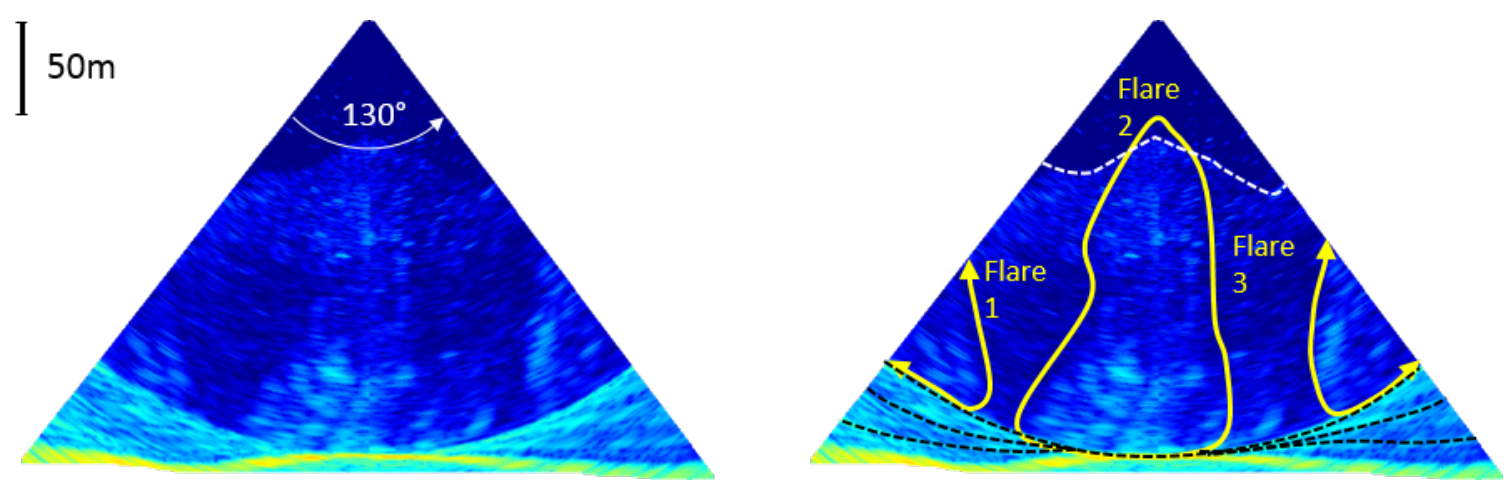

Figure 2.2: Unlabelled (left) and labelled (right) polar echograms for a single ping from the TAN1404 voyage. The echograms are coloured by the received signal strength that is returned from parts of the water column within a 2-dimensional across-track slice. High intensity shades of green to yellow at the bottom of the echogram represent seafloor sediments. Lighter coloured arc-shaped blue regions (highlighted by the broken black lines) directly above the seafloor are side lobe echoes from the adjacent seafloor. The highly-reflective side lobes conceal other reflections (e.g. from bubbles) in the spatial zones that they cover. Above the side lobes are three distinct bubble trails that have been labelled as flares. The two lateral flares appear to extend outside of the beam coverage. The lighter coloured background reflectivity that extends up to the white dotted line is known as a 'deep scattering layer' (Medwin and Clay, 1998).

on the adjacent seafloor. These echoes arrive subsequent to the first arriving seafloor reflection resulting in multiple 'side-lobes' (Chadwick et al., 2014) in both lower corners of echograms (Figure 2.2). Any water column reflections in the region occupied by these side lobes are therefore concealed. To account for these masking effects, we have suppressed the side lobes using different methods in each of the software packages we used.

After suppressing the side lobes, points are then spatially restricted to a desired volume using different methods in each software. Following this, the intensity values of all remaining points within the volume are then vertically summed to produce maps that display signatures of active seepage.

\subsection{Sonarscope}

Water column processing tools available in SonarScope were employed to identify seep locations over the entire survey area. This software has the largest capacity of the three that are used for handling multiple track datasets and integrating them into combined calculations. For this reason, SonarScope has been used to produce regional intensity 
maps, which have been used primarily for finding and counting seeps. Collaboration with Jean-Marie Augustin (developer of the SonarScope Software) also assisted in refining the methods and parametrizations in Sonarsope to produce maps of high precision and minimal noise.

For this project, the side lobe echoes are filtered using a 'side-lobe compensation' tool. This function calculates a mean amplitude for subdivisions of each echogram to construct a mean-intensity function across the echogram's entire extent. The mean function is then subtracted from the original intensity values so that only localised data anomalies (primarily gaseous bodies) remain above the sea floor. Once the data have been cleaned in this way, intensity data from echograms for selected lines are then summed between upper and lower vertical limits (specified as percentage values of the echogram heights). The resulting mosaic of vertically summed intensity can then be draped onto the bathymetry that was recorded for the lines to observe where seepage occurs.

Compensation curves were produced for post-processed data cleaning as a method of reducing the high-amplitude impact of specular reflections. Reflections received from the nadir to the ship have lost minimal energy due to scattering. When vertically summing reflective intensity values, specular reflections are obvious as a comparatively high-amplitude band along the centre of the ship's track. To reduce the amplitude of specular reflections, mathematical trends are produced for lines with no evidence of active venting to acquire mean across-track summed intensity curves of the ambient water column. These curves capture the large proportion of summed energy at the nadir. Subtracting these data curves from the amplitude data of all lines constituting a summed-intensity mosaic effectively filters the specular anomalies. All distinct high intensity zones that remain after successful filtering can then be assumed as seep indicators. This filtering allows for seeps situated nadir to a multibeam echosounder to be easily more identified.

It is important to note that the number of seep indicators displayed in the summed surfaces represent a minimum estimate of the total seep count in the surveyed region. When mapping seeps during TAN1505, the average spacing between lines was $50 \%$ of the base length of the echogram coverage. Figure 2.3 and 2.4 illustrate the geometry of the section of water column and bathymetry imaged by pings of $50 \%$ spacing. Whilst the entire swath of the bathymetry is covered, only $70 \%$ of the rectangular area in Figure 2.4 is imaged (due to the space outside of the ping range and the masking effect of sidelobe echoes). Of the proportion of the imaged water column, most of the coverage occurs in the deeper part of the total depth range. Partial coverage means that many active seeps present along the swath may have only been partially imaged, 
or were undetected. Partial segments of flares captured in echograms will be relatively lower in summed intensity in comparison to wholly image flares. This may result in partially imaged seeps being unnoticeable and easily removed if intensity surfaces are filtered by amplitude. In the final seep count, partially imaged and undetected flares in the un-imaged water column are not included, resulting in an underestimated total number.

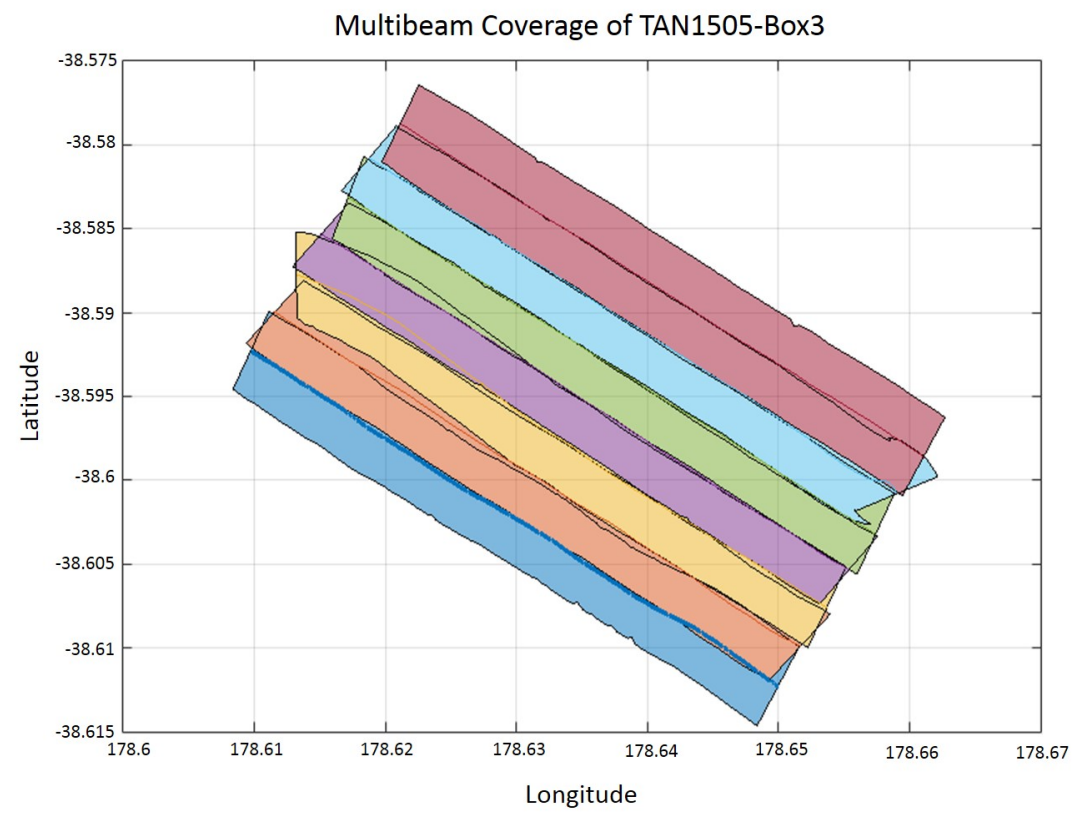

Figure 2.3: Map view of the multibeam coverage for lines of TAN1505 -Box 3 (see Figure 3.2). Here the coverage is approximately $50 \%$ across the width of adjacent swaths.

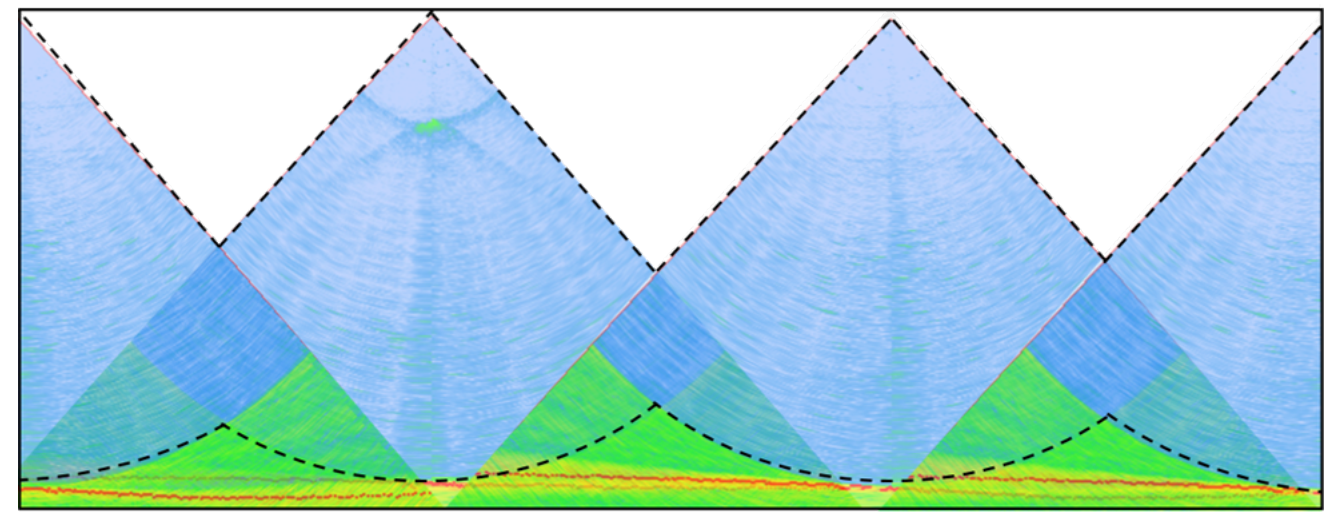

Figure 2.4: Echograms from adjacent lines surveyed over TAN1505 -Box 3 (see Figure 3.2). $50 \%$ coverage means that only water column data with the blue-coloured area (outlined with a dashed line) is collected. Any seep activity in the green and white areas is not imaged. 

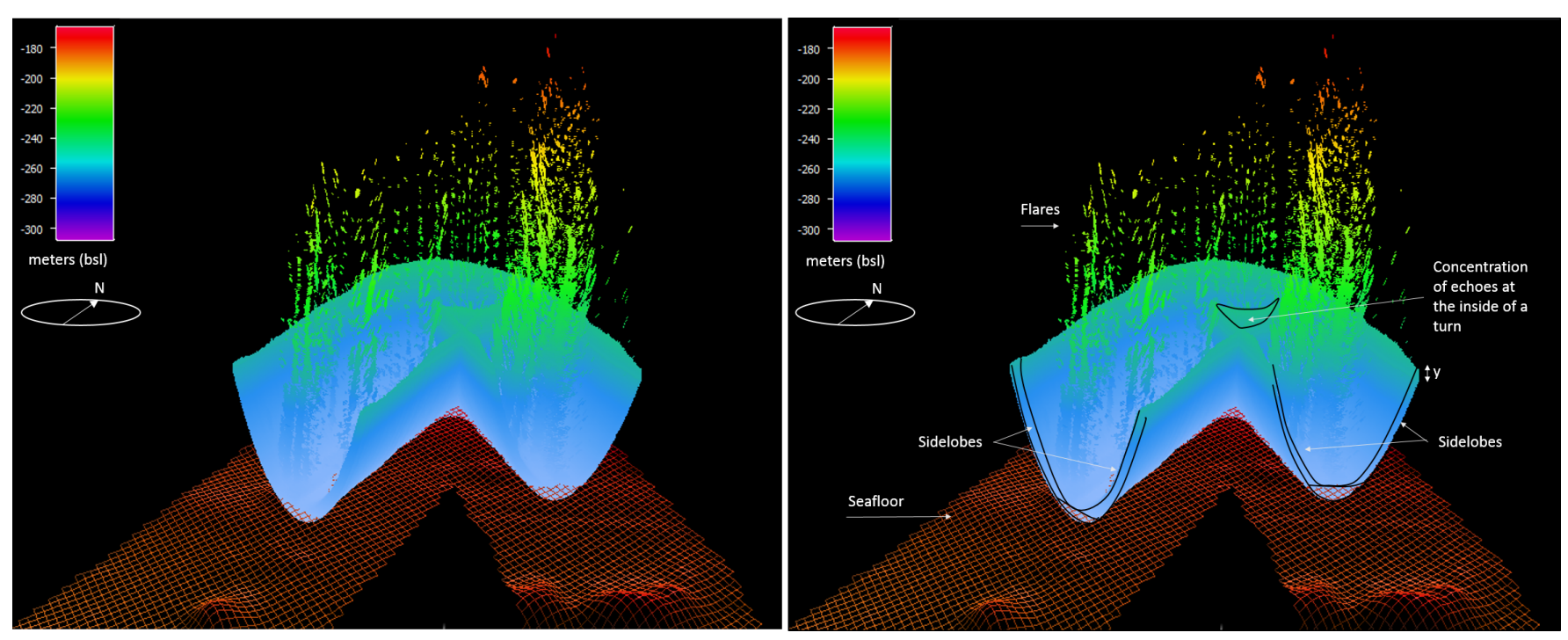

Figure 2.5: Plotted point data (coloured by elevation) for flares over the bathymetry (red grids) of a corner section of the multibeam data displayed in Figure 1.1. Around the flare data are the side-lobe returns. Side-lobe data vertically sums to create erroneous high-amplitude bands along the outside of the ship's track. Side-lobe summing effects are particularly apparent on the inside bends of a turning path of the ship, where the slower movement on the inside of the turn results in a relatively high accumulation of data points.

\subsection{Matlab and Fledermaus}

In order to further refine the parameters of the summation technique, we have used Matlab to write a code (Summed_Intensity_Maps.m, found in Section 6.1) that computes a mosaic of summed values on a single-line scale, similar to the SonarScope method. This is used to investigate high-amplitude zones on regional maps created in SonarScope more closely. Different parts of flares may be separately analysed to infer how much undissolved gas has been imaged at different parts of the water column.

The input files for Summed_Intensity_Maps.m consist of point data with latitude, longitude, depth bsl, and reflection amplitude. For this study, we organised these data in this manner from the raw multibeam .all and .wcd files using Fledermaus MidWater software(FMMidwater), which is an extension of Fledermaus designed for visualising and processing water-column data. These data are selected from the stacked watercolumn view (as in Figure 1.1[A]), for which the vertical profile boundaries, beam numbers and amplitude range can all be specified when exporting the point data. A condition for the Matlab code's sea-floor identification is that the deepest points are those that constitute the side-lobe reflections. This condition can be satisfied by eliminating all high amplitude points below the side-lobes using an adjusted amplitude threshold filter prior to exporting from FMMidwater (as has been done in Figure 


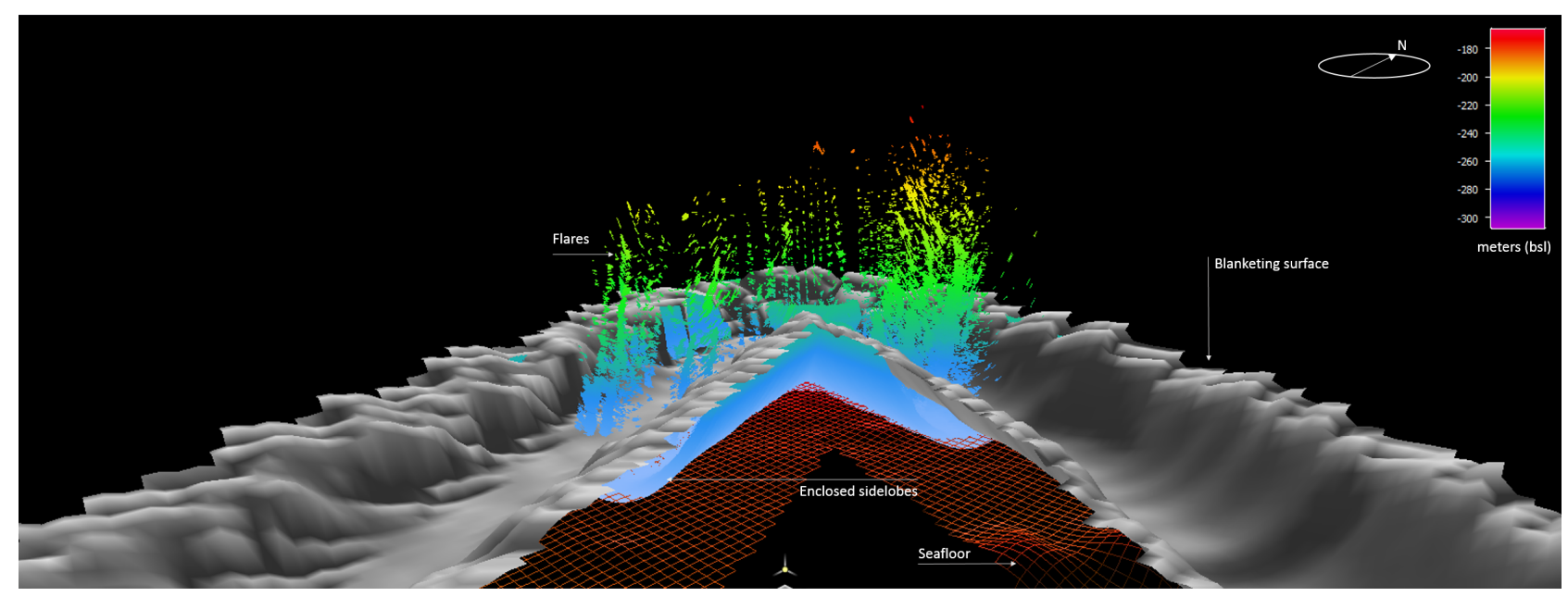

Figure 2.6: Lower bounding surface for vertical summation operations in the Matlab script. Elevations of upper and lower surfaces of depth windows are measured from this basal surface.

$$
1.1[\mathrm{~B}])
$$

The code works by gridding the data-point cloud into square based columns of a specified depth range and grid dimension (where smaller spacing will produce a higherresolution intensity map, but is computationally slower). The lower extent of the data cloud is bound by the side-lobe arcing surface (Figure 2.5). When these data are gridded, it is recognised that the lowest point in each column constitutes the underlying face of the side-lobes (due to the condition stated in the previous paragraph). The thickness of the layer that constitutes the sound front (y on Figure 2.5) is assumed to be constant across the data set. The distance $\mathrm{y}$ is specified in the code by the user after observing the point data side-lobe thickness. A surface (Figure 2.6) is created at a distance y above the lowest points of the data cloud, which acts as a lower bound for all vertical summations. By calculating intensities above this blanketing surface, side lobes are rejected from vertical summations.

High-amplitude data that remain above the surface are likely from gaseous bodies. The amplitudes of these points within each of the grid columns are then summed so that each grid space has a single summed value. The data are then exported to DMagic (an extension of Fledermaus) where the mosaic is interpolated and can then be viewed in Fledermaus.

\subsection{Paridgm-GOCAD and OpendTect}




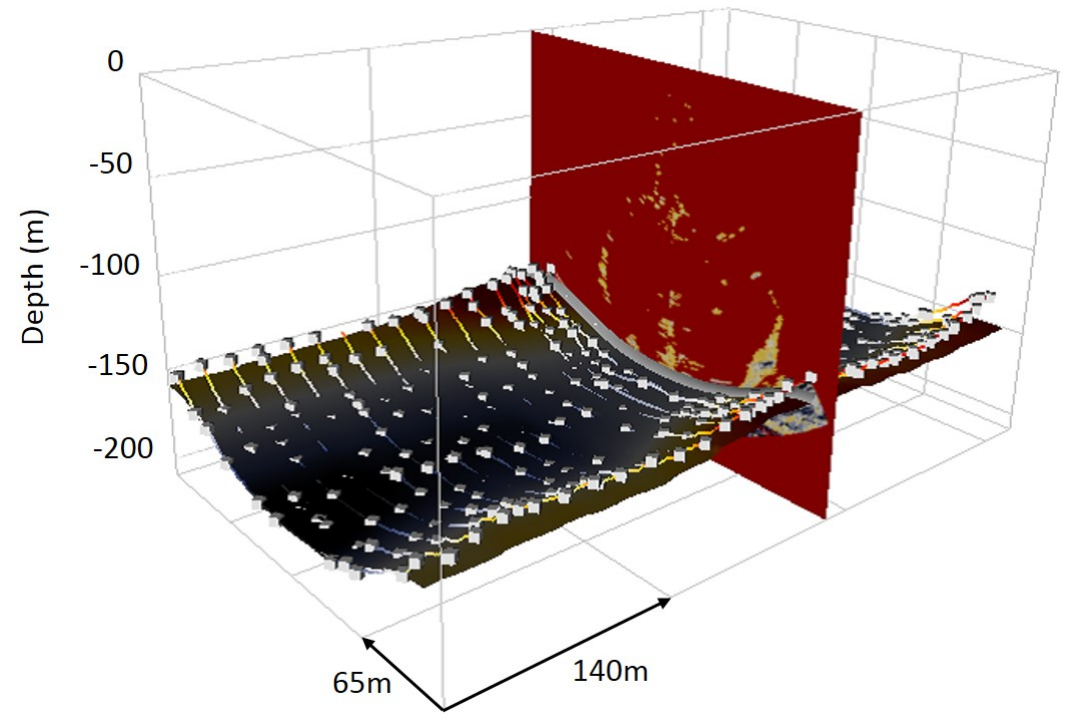

Figure 2.7: A horizon has been generated from multiple picks made on echograms along a section of a line. These picks (white boxes) are at the top of the uppermost side lobe energy. In areas where the bathymetry changes rapidly, closer picking increments are required to adequately sample the surface. A single echogram is displayed part-way across the line.

The Paridigm SKUA-GOCAD software is conventionally used for the modelling and interpretation of seismic data sets. The software has the ability to convert ASCII point data into SEG-Y format, which allows water-column data to be loaded into other seismic processing and seismic interpretation software. We used OpendTect V5.0.4 to perform similar vertical summation calculations and display the summed intensities as seismic attributes. Horizon surfaces were traced and then used as spatial bounds for the summations.

In order to separate the seafloor reflections and side lobes from the overlying water column data, a horizon was created along the upper surface of the highest side lobe (analogous to the blanketing surface in the Matlab method). The horizon is generated by tracing the side-lobe surface on multiple echograms along a desired section of a line (as in Figure 2.7).

Once the picked horizon is saved, it is duplicated at staggered elevations (Figure 2.8) above the original horizon. These staggered horizons are then used as discrete spatial limits for vertical summation of the data contained between the surfaces. The calculated summed intensity values for a discrete volume of data can then readily be viewed as a colour map on the upper or lower horizon to show how the intensity of a flare changes at different levels in the water column. 


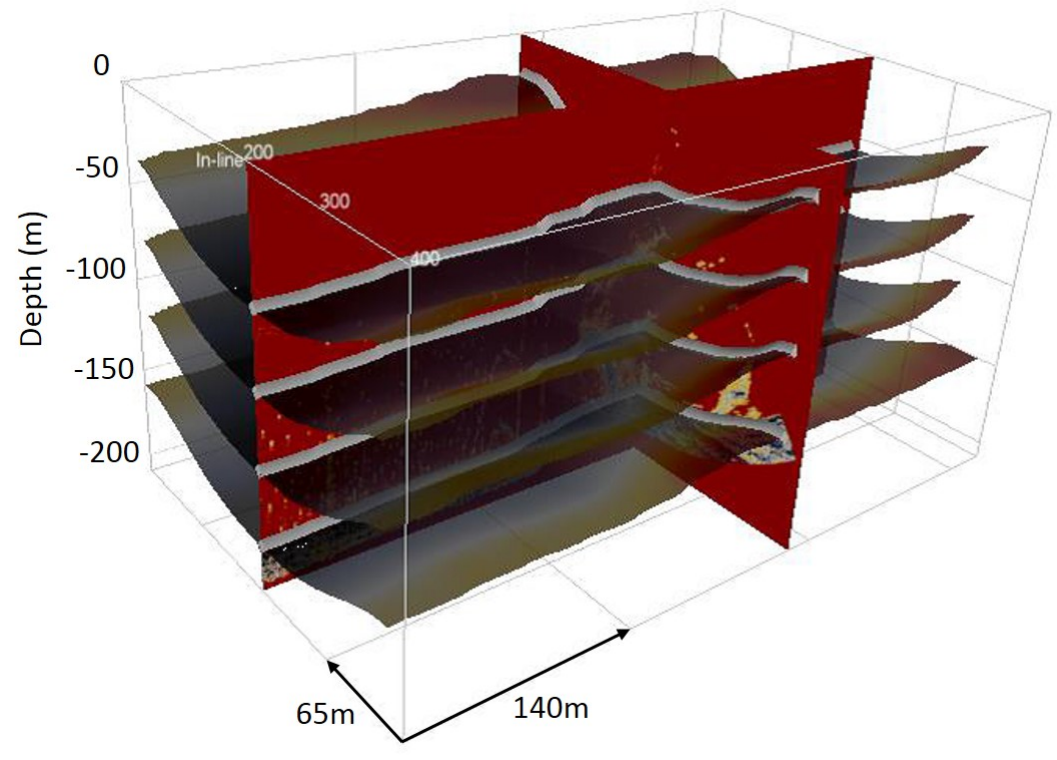

Figure 2.8: The previously generated horizon has been duplicated above the original surface at a $20 \mathrm{~m}$ elevation step sizes. 


\subsection{Bubble size and position measurements}

Determining bubble size and rise rate distributions is integral to calculating the flux at which undissolved methane is being released by seeps. To ensure that these are calculated to a high degree of accuracy, measurement techniques involving Canadian Grids (Wakefield and Genin, 1987) combined with additional geometry (McGovern, 2012) have been applied to still frames of the DTIS camera footage. All bubble-measurement calculations (detailed in the following paragraphs) have been fully automated in Matlab scripts (Section 6.3 - 6.11). These methods relate dimension measured directly on an image (denoted with lower-case variables and termed 'image-plane' measurements) to those measured in the real space in the camera's field of view (denoted with upper-case variable and termed 'scene' measurements). Computationally ordered calculations and user input processes of the Matlab scripts are illustrated in the in the Figure 2.9 flow diagram.

\section{Canadian Grid Geometry}

A Canadian Grid (Figure 2.10) is used as a measuring lattice that extends over a flat plane across the camera's entire field of view (Wakefield and Genin, 1987). For the purpose of measuring bubble dimensions, this plane correlates to the sea floor, which is assumed to be flat within the field of view of the camera.

The grid operates as a scaling tool for image measurements (referred with lower-case letters) and scene measurements (referred with upper-case letters) situated on the seafloor and also functions as a foundation for further geometric calculations in the water column. A Matlab program has been written so that a Canadian grid is constructed for any chosen frame of the DTIS footage (see Section 6.6). The steps for constructing a Canadian grid for a chosen still frame have been followed from Wakefield and Genin (1987) (Figure 2.10). In our method for constructing Canadian Grids, distances between two points are specified with a line above the end-point variables. Canadian Grid calculations are based on the initial known values of (i) the height of the camera above the planar surface $(\overline{\mathrm{ON}})$, (ii) the inclination of the camera's optical axis below the horizontal $(\theta)$, (iii) the camera's horizontal and vertical angles of view ( $\beta$ and $\alpha$ respectively).

For the set-up of DTIS during the TAN1505 voyage, the inclination of the camera $(\alpha)$ 


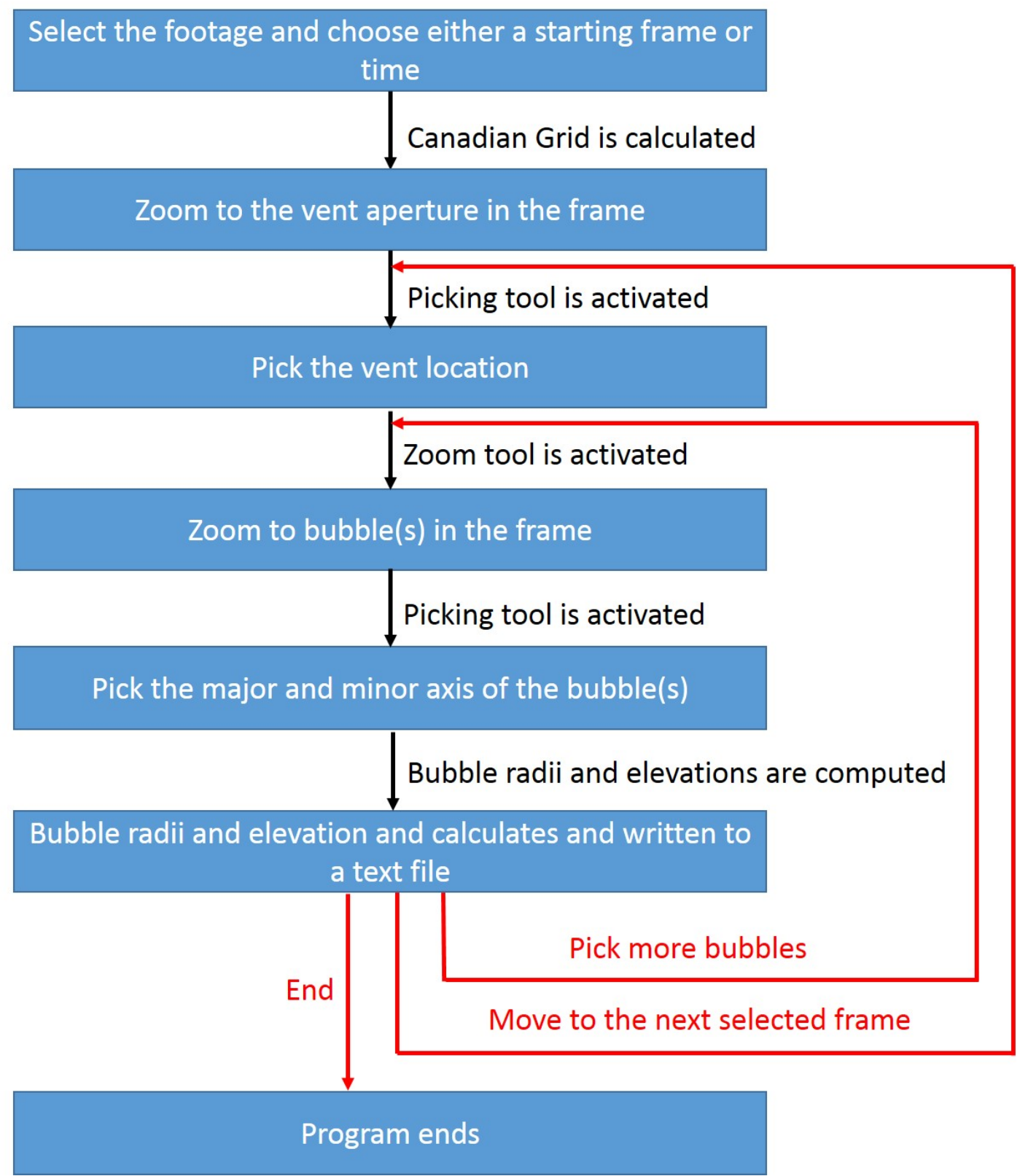

Figure 2.9: Flow diagram showing the ordering of Matlab scripts used for the calculation of bubble size and elevation on still video frames. Blue boxes indicate that user input is required. Black labelled arrows specify automated calculations carried out in Matlab. Red labelled arrows indicate a choice made by the user. 

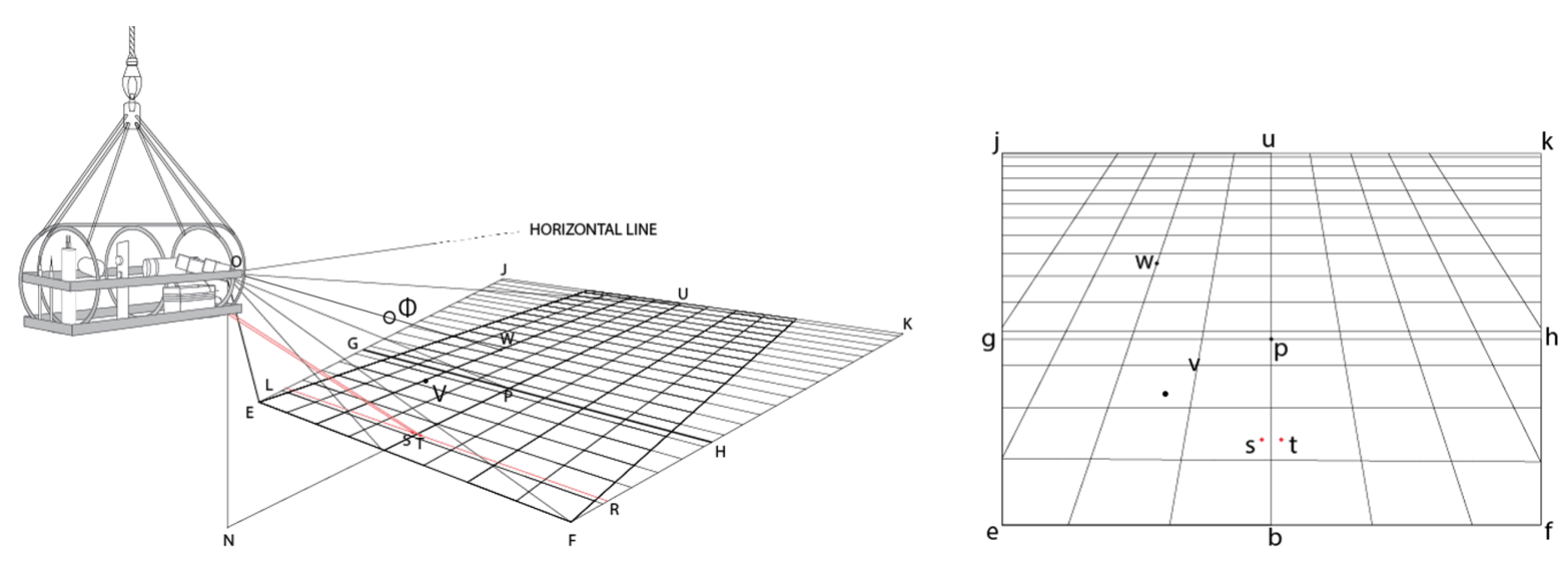

Figure 2.10: Diagrams of Canadian Grid geometry. Left: DTIS camera with a Canadian Grid overlain on the field of view. All points in the 3-dimensional scene are referred to with upper-case letters. Here $\mathrm{V}$ denotes the aperture that emits bubbles, $\mathrm{S}$ and $\mathrm{T}$ are the respective left and right incident laser points and the bubble at $\Phi$ is situated somewhere along the line $\overline{\mathrm{OW}}$. Right: The 3-dimensional scene captured in a 2-dimensional image as in a camera still. The position of the observed points as located on the image are referenced with lower-case letters. Here the line from the origin though the bubble is observed as a point $\mathrm{w}$ on the image plane.

to the suspending cord was set at $45^{\circ}$. Considerable effort was focused on maintaining this as a constant throughout all of the surveys. The ship was kept to a maximum speed of four knots so that minimal acceleration/deceleration to prevent the camera from swinging in a lagging motion.

The continuous rolling motion of the boat induced by swell, primarily translated to a vertical displacement of the camera and did not cause it to tilt on its axis. Any smaller jolting ship movements were also rarely translated to the camera as it was suspended from between 200-300 m of wire that absorbed most of the energy before it was able to disrupt the DTIS. However, to avoid any unnecessary disturbance, the DTIS was not operated in bad weather conditions.

The horizontal and vertical angles of the camera's view are calculated from the camera lens dimensions of the Sony HDRPJ760V. The DTIS has a I/2.88 sensor type wide angle carl zeiss lens (set to maximum angle). The focal length is $3.8-38 \mathrm{~mm}$, which when cropped to a $35 \mathrm{~mm}$ frame equates to $26-260 \mathrm{~mm}$ ( $16 \times 9$ aspect ratio). Trigonometric calculations from the geometry illustrated in Figure 2.11 was used to calculate angles $\alpha$ and $\beta$ of $55^{\circ}$ and $68^{\circ}$ respectively.

Both of these angles were then corrected for the difference between the refractive index of air $\left(\mathrm{n}_{\text {air }}=1.00028\right)$ and water $\left(\mathrm{n}_{\text {water }}=1.334\right)$ (Bashkatov and Genina, 2003)). Eq. 2.1 was used to acquire revised horizontal and vertical angles of $62.8^{\circ}$ and $46.0^{\circ}$ respectively. 


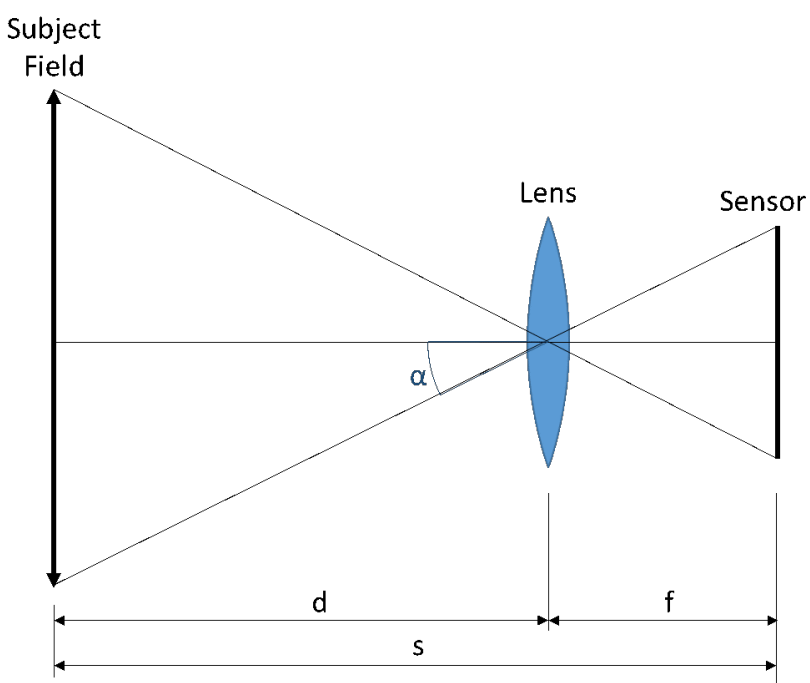

Figure 2.11: Side-view of the geometry that light travels from a subject field to a camera sensor to produce an image. $d$ is measured from the camera lens to the subject field. The focal length $\mathrm{f}$ is measured from the sensor to the lens. $\mathrm{s}$ is the sum of $\mathrm{d}$ and f. $\alpha$ (and perpendicular angle $\beta$, which is not shown in the figure) is the maximum angle that light is able to pass through the lens to be captured in the image.

$$
\alpha, \beta_{\text {water }}=\sin ^{-1}\left(\frac{\sin \left(\alpha, \beta_{\text {air }}\right) \cdot n_{\text {air }}}{n_{\text {water }}}\right)
$$

\section{Pre-Computational Image Corrections for Camera Height}

The elevation of the camera above the seafloor could not be accurately determined from the instrumentation for all individual frames of the video footage. Instead of constraining the results to specific frames where elevation data were recorded, the image spacing of the parallel lasers has been used to derive the camera height. Using laser-spacing also makes the programme applicable to a wider range of camera instrumentation, as all measurement techniques will be derived purely from the video footage, without the need for additional positioning systems. To locate the position of the lasers on the seafloor for each still frame of a video, the incident beams are identified by colour.

For each chosen still frame of the video, a corresponding red, green, blue (RGB) image is loaded into the code sequence as an m-by-n-by-3 matrix (where $\mathrm{m}$ and $\mathrm{n}$ are the respective horizontal and vertical number of pixels for each image). The colour of each pixel is determined by the combination of the RGB intensity values (between 0 and 1) stored in each matrix 'colour' array at each pixel's location. Graphics file formats 
store RGB images as 24-bit images, where the red, green, and blue components are 8 bits each.

The position of each incident laser on the unadjusted RGB image frame is located using the 'laser detector' code (Figure 2.12). This code marks pixels in the red-colour array that have a value over 0.95 . Red pixels that are separate from the laser dots are then rejected by removing any marked pixel that has fewer than 12 other marked pixels within 6 neighbouring columns. The remaining clusters of pixels are then grouped with the left or right laser based on their assorted horizontal coordinate positions. Points in both assemblages are then spatially averaged to acquire central coordinates, locating each laser.

The observed laser spacing $\overline{s t}$ is used to calculate the distance, $\overline{\mathrm{LR}}$ (Figure 2.10). The vertical distance from the base $(\overline{\mathrm{ef}})$ of the horizontal line $\overline{\mathrm{LR}}$ is taken as the mean vertical position of the lasers $(\overline{\mathrm{el}} \simeq \overline{\mathrm{fr}})$. An average of $\overline{\mathrm{el}} \& \overline{\mathrm{fr}}$ is used as the verticallaser position as slight alignment disparities sometimes arise between the two beams due to either (i) slight bathymetry gradients (ii) twisting of the camera around the horizontal axis as a result of the ship making a turn.

The primary steps in creating Canadian grids involve evaluating true distances across the camera's field of view (Figure 2.13). The length of $\overline{\mathrm{LR}}$ is first measured using the pixel distance $\overline{\mathrm{st}}$ as a $20 \mathrm{~cm}$ scale as in Eq.2.2

$$
\overline{\mathrm{LR}}=\overline{\mathrm{ef}} \overline{\mathrm{ST}} / \overline{\mathrm{st}}
$$

Here, (from Figure 2.10) $\overline{\text { ef }}$ is the horizontal distance across the image plane, $\overline{\mathrm{ST}}$ is the true laser spacing (20 cm in the case of NIWA's DTIS) and $\overline{\mathrm{st}}$ is the number of pixel in the horizontal distance between the two located laser points.

The height of the camera $(\overline{\mathrm{ON}})$ is then obtained using Eq.2.3

$$
\overline{\mathrm{ON}}=\overline{\mathrm{LR}} \sin \theta-\gamma /[2 \tan (\beta / 2))]
$$

Here, $\gamma$ is the angle of the laser to the centre of the camera's field of view (at an angle $\theta$ in Figure 2.15). This is calculated using Eq. 2.4 from the triangle $\angle$ Opj between the 

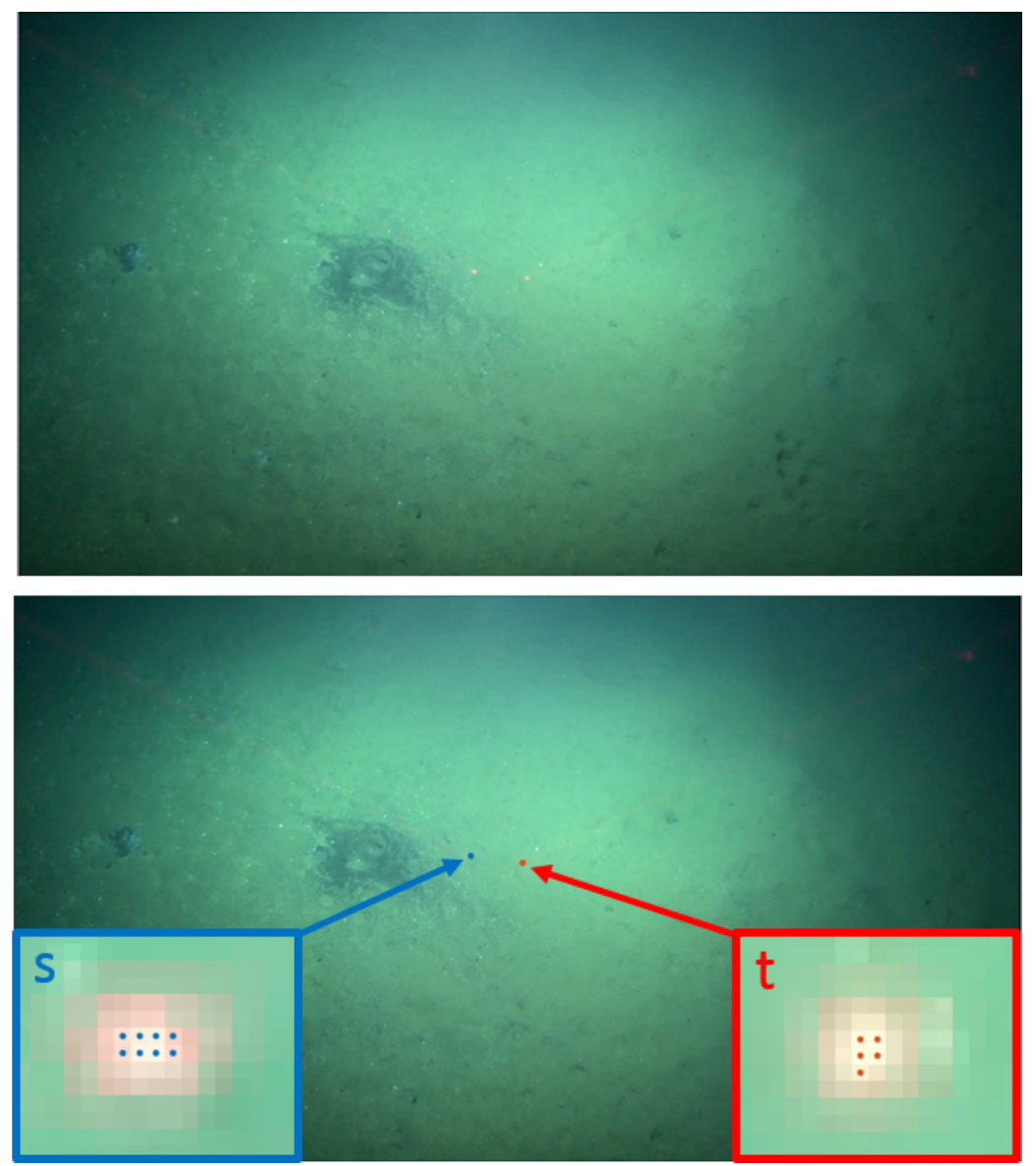

Figure 2.12: Laser detect module. Top: DTIS image. Bottom: Pixels with a red component of over 0.95 have been market. These have then been separated between the left and right laser points. The points $\mathrm{s}$ and $\mathrm{t}$ denote the left and right laser respectively in geometric calculations. 

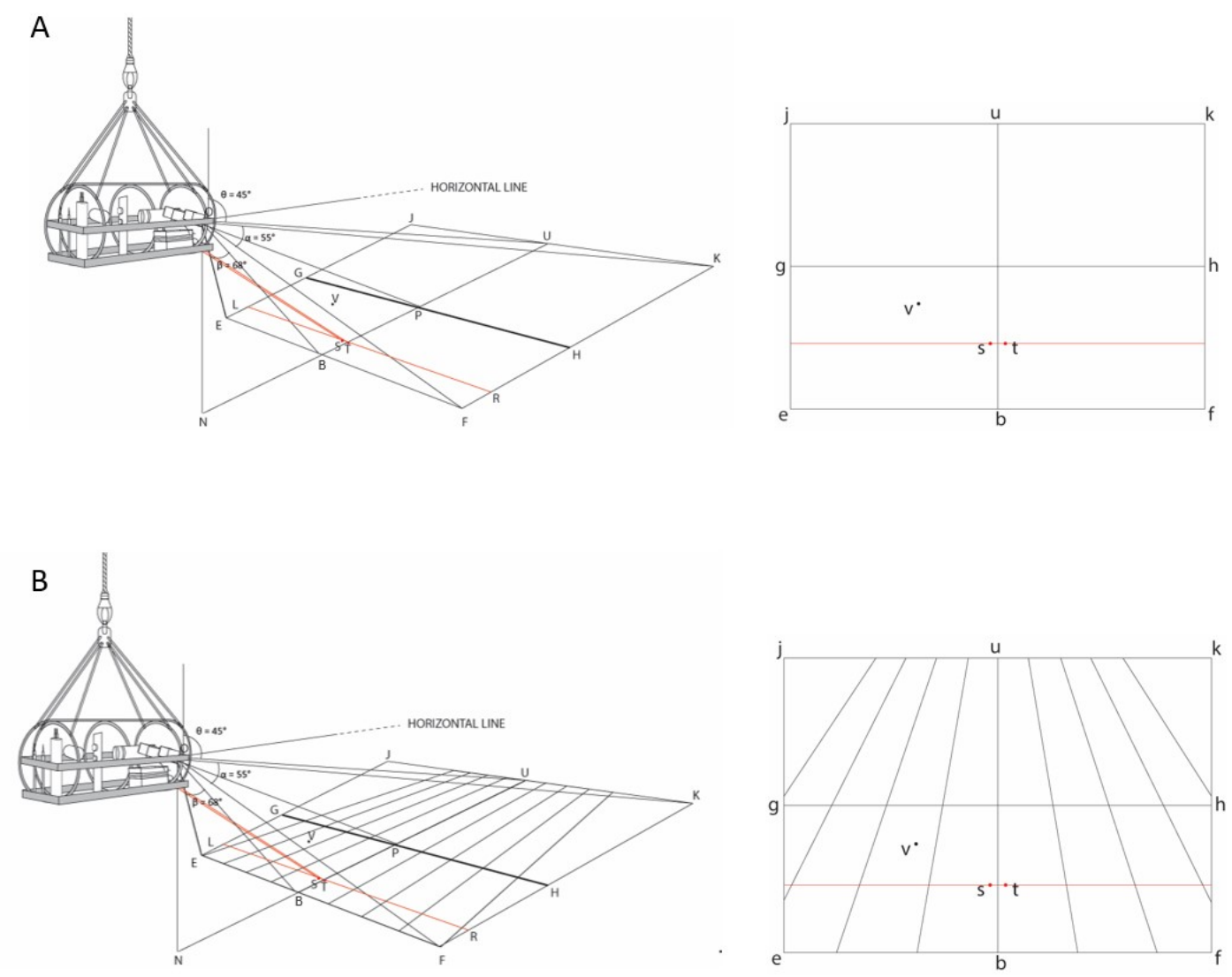

Figure 2.13: Intermediate steps in generating Canadian Grids. 
origin and image plane (Figure 2.15).

$$
\gamma=\tan ^{-1}\left(\frac{\overline{\mathrm{ef}}}{2-\overline{\mathrm{el}} / \overline{\mathrm{Op}}}\right)
$$

With the height of the camera acquired, it is then possible to calculate the true length of the base line $(\overline{\mathrm{EF}})$ from Eq.2.5

$$
\overline{\mathrm{EF}}=2 \overline{\mathrm{OB}} \tan (\beta / 2)
$$

Here, the distance from the camera to the base line $(\overline{\mathrm{OB}})$ is equated using Eq. 2.6.

$$
\overline{\mathrm{OB}}=\overline{\mathrm{ON}} / \sin \theta+\alpha / 2
$$

Once lines $\overline{\mathrm{EF}}$ and $\overline{\mathrm{OB}}$ have been evaluated, the 3-dimensional scene distances are related to the perceived image measurements along $\overline{\mathrm{lr}}$ and $\overline{\mathrm{ef}}$ using scaling factors termed $\mathrm{Sh}_{\mathrm{j}}$ (Eg. 2.7) and $\mathrm{Sh}_{\mathrm{b}}$ (Eq. 2.8) respectively.

$$
\begin{aligned}
\mathrm{Sh}_{\mathrm{j}} & =\overline{\mathrm{lr}} / \overline{\mathrm{LR}} \\
\mathrm{Sh}_{\mathrm{b}} & =\overline{\mathrm{ef}} / \overline{\mathrm{EF}}
\end{aligned}
$$

The meridian grid lines are then resolved by joining lines $\overline{\mathrm{EF}}$ and $\overline{\mathrm{OB}}$ at equidistant points (as has been done in Figure 2.13[B]). The chosen horizontal spacing between joining lines determines the grid-square size of the Canadain Grid.

The spacing between horizontal grid lines decreases with increasing image distance the baseline (Figure 2.10). The image distance from the base to a horizontal line, $(\overline{\mathrm{ba}})$ is evaluated for chosen scene grid intervals $(\overline{\mathrm{BA}})$ using Eq. 2.9.

$$
\overline{\mathrm{ba}}=\overline{\mathrm{bp}}\left[1+\frac{\tan (\theta-\arctan (\overline{\mathrm{ON}} /\{\overline{\mathrm{BA}}+\overline{\mathrm{ON}} \cot (\theta+\alpha / 2)\})}{\tan (\alpha / 2)}\right]
$$




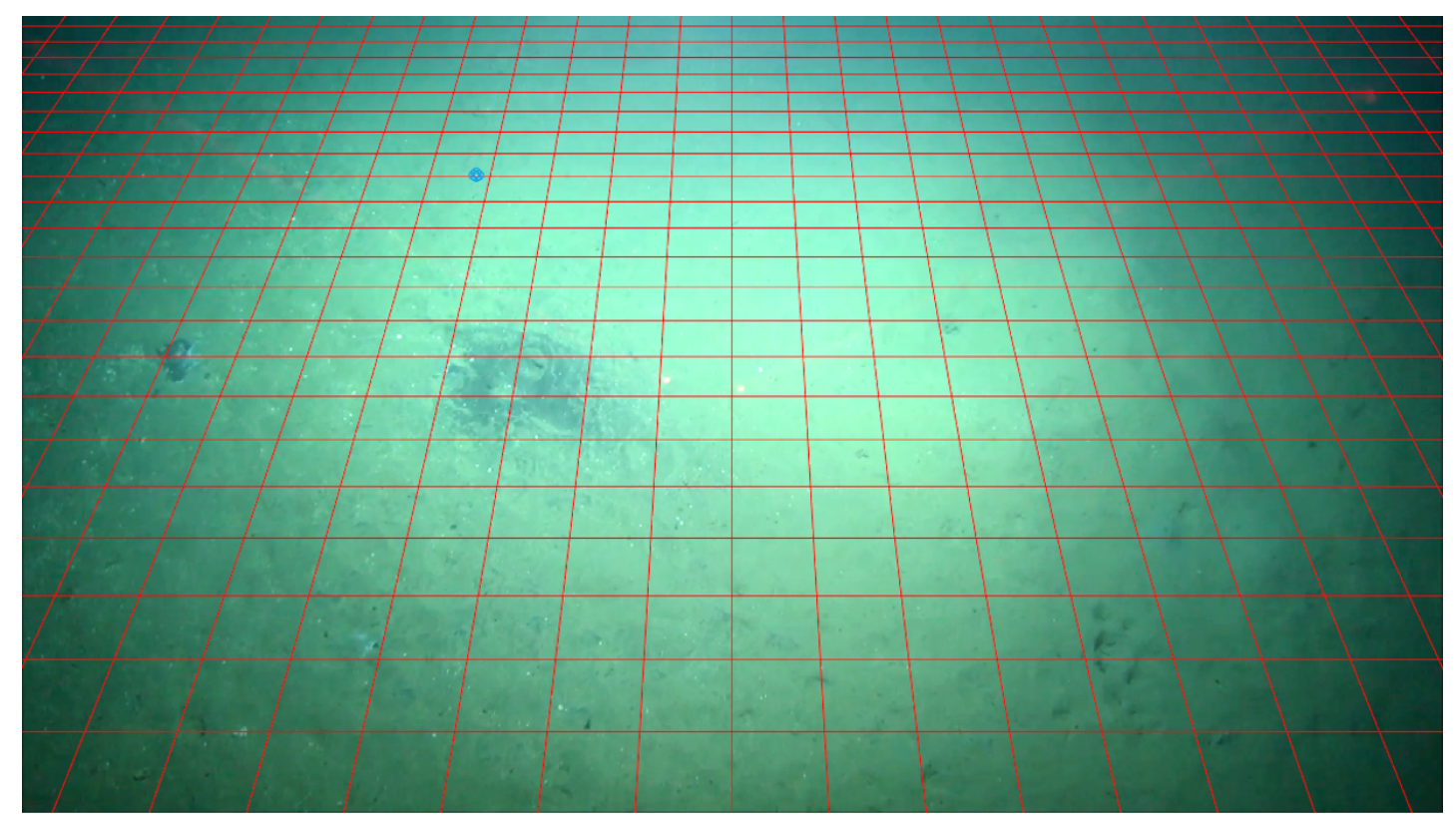

Figure 2.14: An example Canadian grid of $20 \mathrm{~cm}$ horizontal and vertical spacing has been constructed using the automated Matlab program for the Figure 2.12 image.

These steps are carried out in the automated program once the user has specified the time or frame number of a loaded video. An example outputted grid is displayed in Figure 2.14.

\section{Bubble rise paths}

The radius and elevation above the seafloor of each bubble is calculated using additional geometry that has been adapted from McGovern (2012) (Figure 2.15). For bubble size calculations, the critical assumption has been made that all bubbles rise in the plane that extends vertically up from $\mathrm{V}$ and stretches horizontally across the camera's field of view. This simplified assumption is made as any movement towards or away-from the camera as a bubble rises cannot be quantified. Only deviations from a straight advection pathway occurring across the field of view can be accurately measured. These distances are later used to predicting the uncertainty of this assumption (Section 2.9).

\section{Locating the point of gas escape}

The initial steps for constructing the above-seafloor framework require that the seafloor aperture $(\mathrm{v})$ be located on the image plane. To satisfy this, the seafloor aperture is 


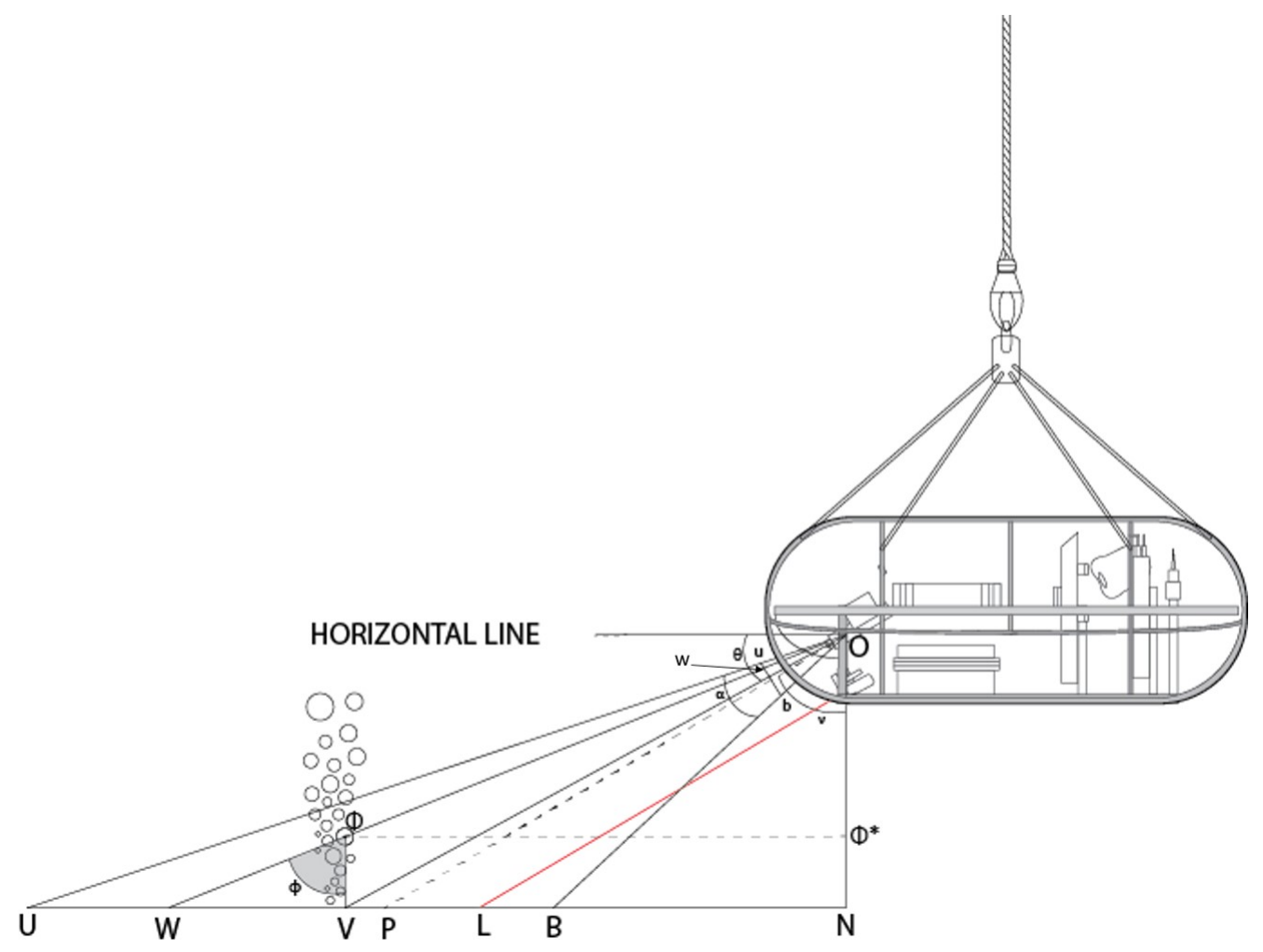

Figure 2.15: Lateral view of the camera and bubble geometry (altered after McGovern 2012). The horizontal axis shows the seafloor, labelled in the same convention as in Figure 2.10 (altered after Wakefield and Genin (1987)). The instantaneous position and angular size of the bubble are labelled $\Phi$ and $\phi$ respectively. 
manually 'picked' in a GUI interface of the selected frame. The pixel coordinates of the aperture are then stored as a Matlab variable.

\section{Locating bubbles}

The surface boundaries for all bubbles in a frame are 'picked' in a GUI interface (Figure 2.16) similar to the seafloor aperture. Preliminary measurements and other documentation (Greinert and Nützel, 2004; Leifer and Culling, 2010) of standard submarine seep bubble-size distributions have confirmed that the majority of bubbles are of a sufficient volume (between $1.5 \mathrm{~mm}$ and $20 \mathrm{~mm}$ equivalent-spherical diameter) to assume that seep bubbles are ellipsoidal (Ellingsen and Risso, 2001; Bhaga and Weber, 1981). Ellipsoidal bubbles of this size range oscillate as they ascend through the water column. Smaller bubbles of diameters ranging from $0.2 \mathrm{~mm}$ to $1.5 \mathrm{~mm}$ are stable in the spherical regime and will follow a straight vertical rise path. To best account for the diversity in bubble proportions, each end of the minor and major axis $\left(\mathrm{s}_{1}(\mathrm{x}, \mathrm{y})\right.$ to $\mathrm{s}_{2}(\mathrm{x}, \mathrm{y})$ and $\mathrm{s}_{3}(\mathrm{x}, \mathrm{y})$ to $\mathrm{s}_{4}(\mathrm{x}, \mathrm{y})$ respectively (Figure 2.17)) is picked for bubbles as observed in the image plane. The position of the bubble in the image plane $(\mathrm{w})$ is then determined from the spatial average of the four axis end points. 

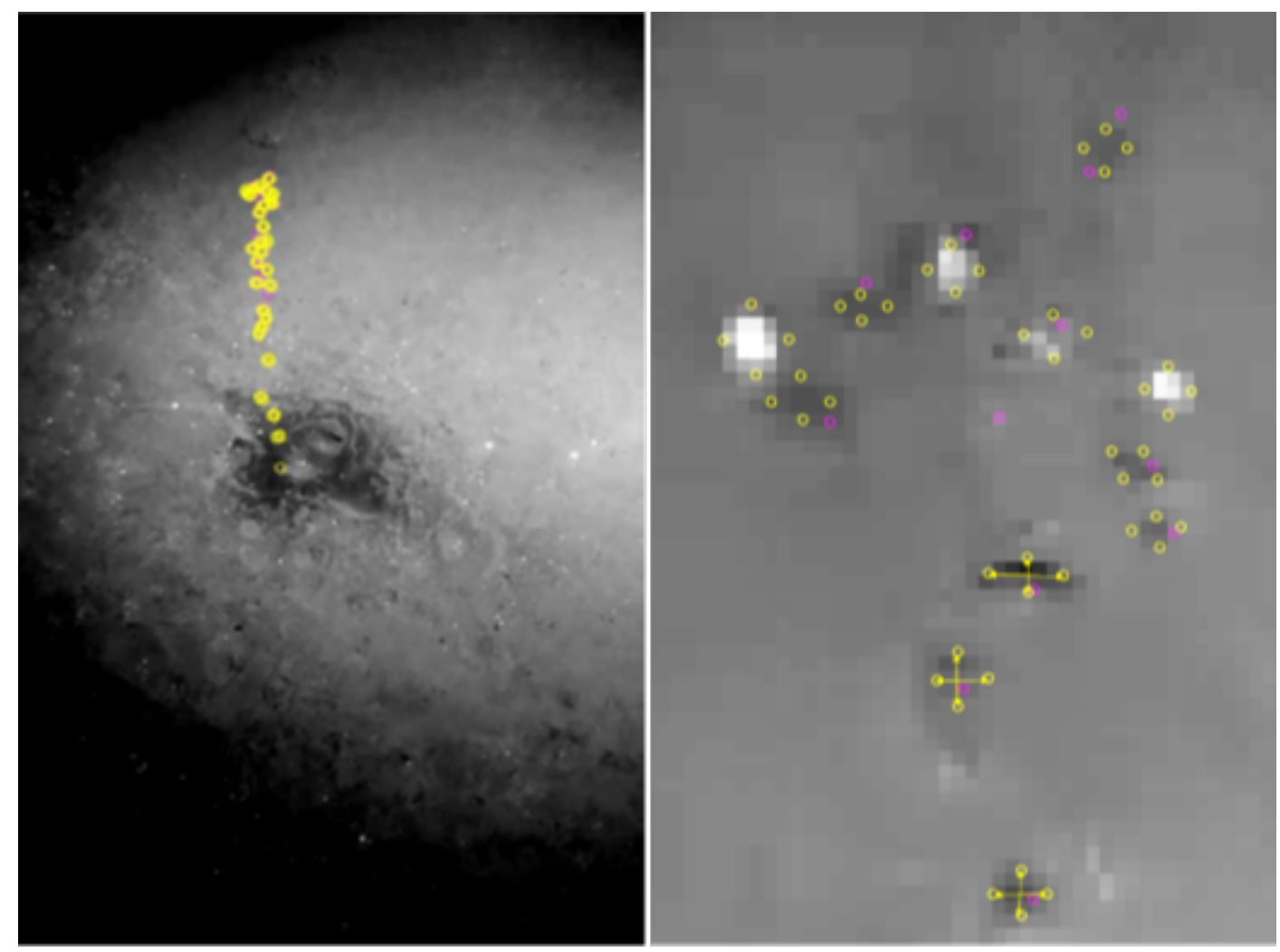

Figure 2.16: Left: Picked bubbles of a seep plume on a DTIS still frame. Right: Zoomed section of the image, where each of the four axes-picks can be seen. In some cases the trace of the major and minor axes have been drawn on the bubble.

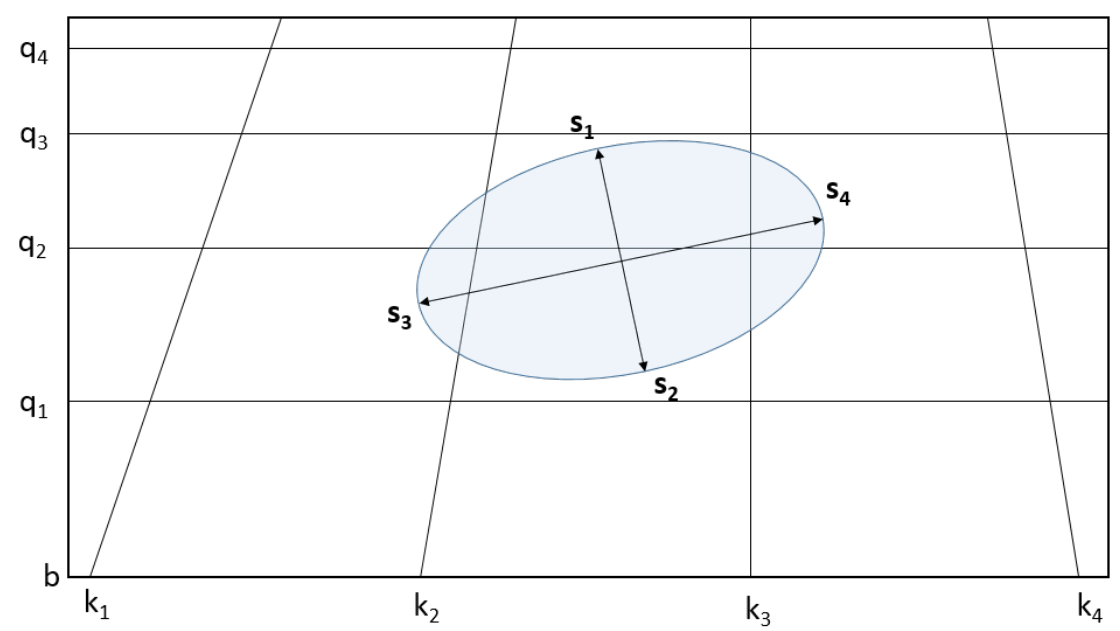

Figure 2.17: An example Canadian Grid of $20 \mathrm{~cm}$ horizontal and vertical spacing has been constructed using the automated Matlab program for the Figure 2.12 image. 


\section{Bubble height}

The elevation of a bubble above the seafloor $(V \Phi)$ is calculated using the image distances (i) $\overline{\mathrm{bv}}$, from the base line to the seafloor aperture (ii) $\overline{\mathrm{bu}}$, from the base line to the top of the image, and (iii) $\overline{\mathrm{bw}}$, from the base line to the bubble centre.

The angles $\nu$ and $\psi$ are calculated using Eq. 2.10 and Eq. 2.11 respectively.

$$
\begin{aligned}
& \nu=\frac{\pi}{2}-\left(\theta+\frac{\alpha}{2}\right)+\frac{\overline{\mathrm{bv}}}{\overline{\mathrm{bu}}} \cdot \alpha \\
& \phi=\frac{\pi}{2}-\left(\theta+\frac{\alpha}{2}\right)+\frac{\overline{\mathrm{bw}}}{\overline{\overline{\mathrm{bu}}}} \cdot \alpha
\end{aligned}
$$

The elevation of a bubble (Vథ) is then calculated from Eq. 2.12.

$$
V \Phi=\frac{\overline{\mathrm{ON}}(\tan \phi-\tan \nu)}{\tan \phi}
$$

\section{Bubble Size}

The true dimensions of each bubble are determined by resolving the position of the bubble along the line $\overline{\mathrm{wW}}$ (See Figure 2.15). The bubble position is then used as a reference to proportionately scale between the maximum and minimum apparent theoretical sizes for the cases of the bubble being positioned at $\mathrm{W}$ or $\mathrm{w}$ respectively.

The maximum theoretical length of the half axes $\left(\mathrm{A}_{1}\right.$ and $\left.\mathrm{A}_{2}\right)$ are calculated for a bubble hypothetically positioned at the seafloor. These dimensions are computed directly from the Canadian Grid lines. The minimum theoretical length of the half axes $\left(a_{1}\right.$ and $\left.a_{2}\right)$ are alternatively calculated for a bubble hypothetically positioned at the image plane and measured in pixels.

Figure 2.17 exemplifies a typical bubble that spans the boundaries of multiple grid spaces. The following procedure was developed to measured bubbles of any size and orientation.

The maximum axes lengths are first computed by finding the scene positions of each of the axis end-point $\left(\mathrm{S}_{1}, \mathrm{~S}_{2}, \mathrm{~S}_{3}, \mathrm{~S}_{4}\right)$. The $\mathrm{Y}$ positions of the axes for each of the theoretical 
bubbles $\left(\mathrm{S}_{\mathrm{n}}(\mathrm{Y})\right)$ positioned at the seafloor are acquired by rearranging Eq.2.9 to obtain Eq. 2.13

$$
\overline{\mathrm{BS}_{\mathrm{n}}(\mathrm{Y})}=\frac{\overline{O N}}{\tan \left[\theta-\tan ^{-1}\left(\frac{\mathrm{b} \mathrm{s}(\mathrm{y})}{\overline{\mathrm{bp}}}-1\right) \cdot \tan \left(\frac{\alpha}{2}\right)\right]}-\overline{O N} \cdot \cot \left(\theta+\frac{\alpha}{2}\right)
$$

In the case of Figure 2.17, $\mathrm{n}=\{1,2,3,4\}$. The total $\mathrm{Y}$ component across the length of each bubble axis axes can then be calculated by finding the differences in Eq. 2.14 and Eq. 2.15.

$$
\begin{aligned}
& \mathrm{S}_{1} \mathrm{~S}_{2}(\mathrm{Y})=\mathrm{S}_{1}(\mathrm{Y})-\mathrm{S}_{2}(\mathrm{Y}) \\
& \mathrm{S}_{4} \mathrm{~S}_{3}(\mathrm{Y})=\mathrm{S}_{4}(\mathrm{Y})-\mathrm{S}_{4}(\mathrm{Y})
\end{aligned}
$$

The length between the axis end-points in the $\mathrm{X}$ direction is determined from the spacing between the meridian grid lines adjacent to the bubble boundaries. The true distance between two neighbouring meridian grid lines is equivalent to the chosen grid spacing for horizontal measurements. Using this information the total length of each long and short axis is then computed using Pythagoras' Theorem in Eqs. 2.16 and 2.17 respectively.

$$
\begin{aligned}
& A_{1}=\frac{\sqrt{\left[S_{3}(X) S_{4}(X)\right]^{2}+\left[S_{3}(Y) S_{4}(Y)\right]^{2}}}{2} \\
& A_{2}=\frac{\sqrt{\left[\mathrm{S}_{1}(X) S_{2}(X)\right]^{2}+\left[S_{1}(Y) S_{2}(Y)\right]^{2}}}{2}
\end{aligned}
$$

To calculate the minimum bubble radii in the image plane, pixel distances are obtained directly from the frame. Similarly Pythagoras' Theorem is used to calculate the total long and short axis distances (Eqs.2.18 and 2.19).

$$
\mathrm{a}_{1}=\frac{\sqrt{\left[\mathrm{S}_{3}(\mathrm{x}) \mathrm{S}_{4}(\mathrm{x})\right]^{2}+\left[\mathrm{S}_{3}(\mathrm{y}) \mathrm{S}_{4}(\mathrm{y})\right]^{2}}}{2}
$$




$$
\mathrm{a}_{2}=\frac{\sqrt{\left[\mathrm{S}_{1}(\mathrm{x}) \mathrm{S}_{2}(\mathrm{x})\right]^{2}+\left[\mathrm{S}_{1}(\mathrm{y}) \mathrm{S}_{2}(\mathrm{y})\right]^{2}}}{2}
$$

The true major and minor bubble axes lengths $\left(r_{1}\right.$ and $\left.r_{2}\right)$ are then resolved between the maximum and minimum theoretical bubble radii using Eq.2.20 (McGovern, 2012).

$$
\left(r_{1}, r_{2}\right)=\left(a_{1}, a_{2}\right)+\left[\left\{\left(A_{1}, A_{2}\right)-\left(a_{1}, a_{2}\right)\right\} \cdot \frac{\overline{\mathrm{wW}}-\overline{\mathrm{W} \Phi}}{\overline{\mathrm{wW}}}\right]
$$

Here, the length of line $\overline{\mathrm{wW}}$, is evaluated using Eq. 2.21.

$$
\overline{\mathrm{wW}}=\frac{\overline{\frac{\mathrm{ON}}{\cos \phi}}-\frac{\overline{\mathrm{Op}}}{\cos \left(\phi-\left[\frac{\pi}{2}-\theta\right]\right)}}{\cos \lambda}
$$

Eq.2.22 is then used to calculate the equivalent spherical radius of ellipsoidal bubbles (McGovern, 2012).

$$
r_{e}=\sqrt[3]{\left(a_{1}^{2} \cdot a_{2}^{2}\right)}
$$

Once all the bubbles in a still-frame have been picked, the values for equivalent spherical radii are normalised to the entry of the highest frequency. The normalised bubble-size distribution (BSD) is then ordered from least to most commonly occurring and written to a text file.

\section{Bubble-Rise Rates}

There is much documentation of experimental bubble radius rise-rate distributions (Woolf and Thorpe, 1991; Woolf, 1993; Leifer and MacDonald, 2003; Leifer and Patro, 2002; Merlivat and Memery, 1983; Mendelson, 1967) for various physical bubble and water conditions. In order to determine which parameters are best suited to those at the shallow seep sites, velocity measurements were made for distinct bubbles that remained clearly visible throughout their ascent.

Velocity values were obtained for individual bubbles by obtaining elevation values every 5 frames from the time of emanation from the seafloor until they leave the video frame. 
It was ensured that the same bubble was tracked across the frame intervals by also recording and plotting the radius of the bubble. The rise rate of each bubble was then calculated by dividing the change in bubble elevation by the time step (frame rate multiplied by the number of frames).

\section{7 $\quad$ Singlebeam acoustic sampling}

Flux calculations in the FlareFlow module require a 3-dimensional model of the echo returns from bubbles that constitute seep bubble plumes. These are obtained from Singlebeam Echo Sounder data that has been read into the Matlab programme 'Echo Analysis'. The research software is conventionally used by NIWA for fisheries stock assessment, but has been modified by Yoann Ladroit (NIWA) for the purpose of extracting flare profiles.

EchoAnaysis graphically displays singlebeam raw data files in an echogram view. These are constructed for each line from the consecutive traces of each ping and colour coded to the amplitude of the signal in dB. Figure 2.18 shows acoustic data that have been filtered between -60 and $-35 \mathrm{~dB}$ to isolate bodies of a density range representative of flare bubbles. Collections of fish and plankton can also be seen in the echogram.

The frequency response of the filtered data is plotted for operating frequencies of 18000 , 38000, 70000, 120000 and $200000 \mathrm{~Hz}$ (Figure 2.19). The highest bubble-frequency response was chosen as a representative frequency that has best captured the size range of the constituent seep bubbles. The positive spike in the returned energy arises from the insonification of bubbles at the correct wavelength of acoustic energy (Barr and Coombs, 2005; Forsberg et al., 2000; Newhouse and Shankar, 1984; Shankar et al., 1986; Urick, 1967).

Flares are selected in Echo Analysis by drawing polygon boundaries that enclose the seep bubble returns. These are viewed in a 'Flare Editor' window (Figure 2.20) that shows the spatial distribution of scattering in 3-dimensional space. These data are corrected using the information of the electrical angles recorded with the EK60 system (by implementing the split-beam technique, see Medwin and Clay (1998)). Points that do not belong to the flare structure that are contained in the selected region are then discarded using Matlab-figure 'select data' tool. For the remaining group of points 


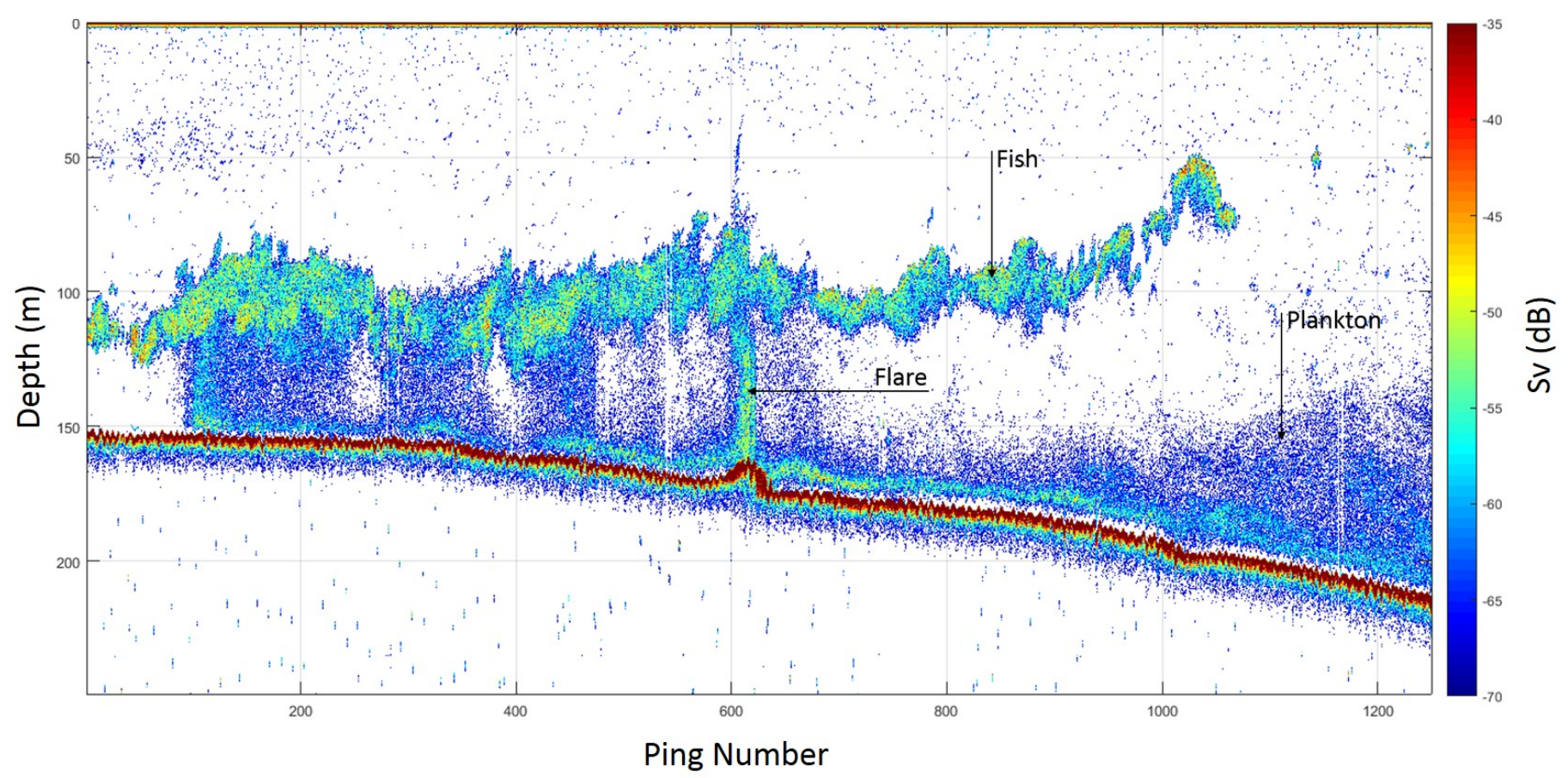

Figure 2.18: 2D cross-section of reflectivity across a single-beam echo sounder profile viewed in EchoAnalysis. The points are coloured by their surface-volume strength, which has been filtered between values of -70 and $-35 \mathrm{~dB}$.

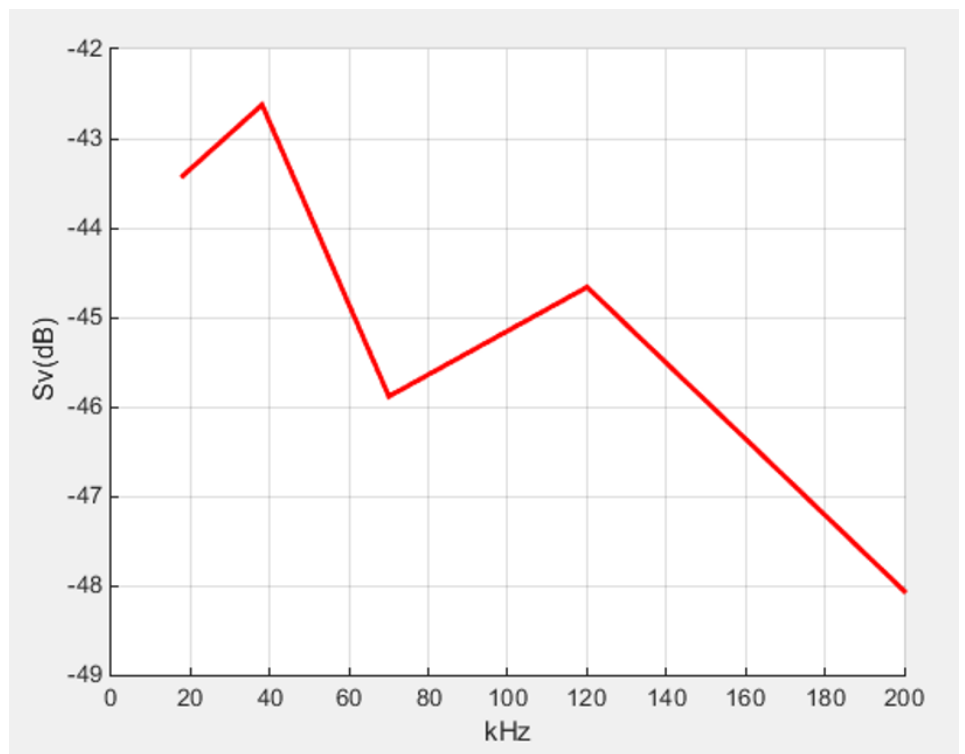

Figure 2.19: Plotted bubble-frequency response of singlebeam acoustic data, filtered to contain flare echoes. The response is highest at $38 \mathrm{kHz}$. 


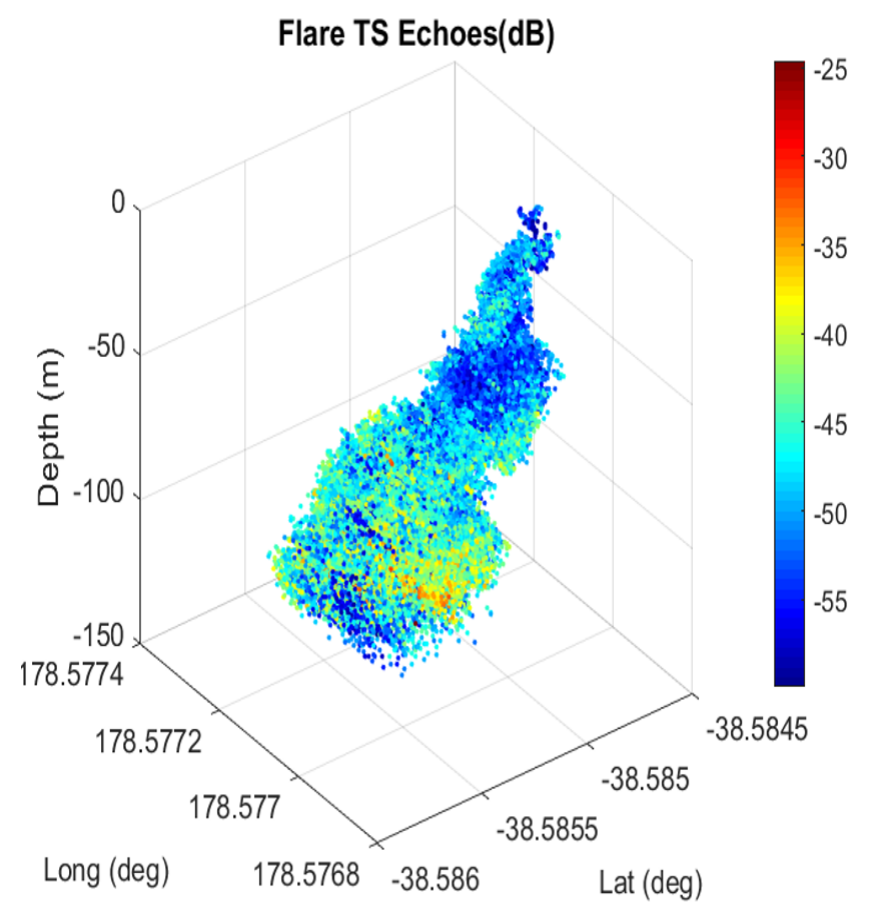

Figure 2.20: $3 \mathrm{D}$ view of selected reflective points of a flare picked up on the singlebeam echo sounder, viewed in Echo Analysis. The points are coloured by their target strength (TS).

in Figure 2.20, the returns have been coloured by their corresponding target strength $(T S)$. This is the logarithmic version of the total backscattering cross-section $\left(\Delta \sigma_{b s}\right)$ of the scatters in the insonified volume (Eq. 2.23) (Medwin and Clay, 1998).

$$
\mathrm{TS}=10 \log \Delta \sigma_{\mathrm{bs}}
$$

Since the data has been manually cleaned so that the remaining points are mostly from seep-bubble echoes, the total backscatter from a sample volume can be divided into contributions from the differently-sized bubbles (Eq. 2.24)

$$
\Delta \sigma_{\mathrm{bs}}=\mathrm{N}_{1} \sigma_{\mathrm{bs} 1}+\mathrm{N}_{2} \sigma_{\mathrm{bs} 2}+\ldots+\mathrm{N}_{\mathrm{n}} \sigma_{\mathrm{bsn}}=\sum_{\mathrm{i}}^{\mathrm{n}} \mathrm{N}_{\mathrm{i}} \sigma_{\mathrm{bsi}}
$$

where $\sigma_{b s i}$ represents the backscattering cross section $N_{i}$ bubbles, where each one has a size $i$ within the sample volume. 


\subsection{Methane flux calculations}

The flux of undissolved methane emitted from an active seep is calculated using the inverse hydroacoustic method, devised and automated in the Matlab programme 'FlareFlowModule' by Mario Veloso (Veloso et al., 2015). A value is obtained in $\mathrm{mL} / \mathrm{min}$ by correlating bubble-size and rise-rate distributions to calibrated single-beam acoustic profiles for the physical conditions at the vent and universal constants of the undissolved gas.

Singlebeam flare profiles are loaded directly into the flux calculator. The point data can then be vertically cropped to a specified depth window for estimating flux values of specific segments of the flare. The remaining data represent bubble echoes of a size range that was able to be insonified by the echosounder frequency. Eq.2.25 from Thuraisingham (1997) expresses $\sigma_{b s}$ in terms of bubble radius $(r)$ and wave number $(k)$

$$
\sigma_{b s}=\frac{r^{2}}{\left[\left\{\left(\frac{r_{0}}{r}\right)^{2}-1\right\}^{2}+\delta\left(r, f_{\text {echo }}\right)^{2}\right] \cdot \frac{(\sin k r / k r)^{2}}{1+(k r)^{2}}}
$$

Here, $r_{0}$ is the bubble resonant radius at an echosounder frequency and specific static pressure. $f_{\text {echo }}$ is the echosounder frequency and $\delta$ is the dimensionless damping (Veloso et al., 2015).

Once a range of bubble radii that have been detected for the chosen frequency have been quantified, the remaining bubbles outside of the insonification detection window are estimated using a probability density function. This is computed from a polynomial fit (of a chosen order) of the normalised BSD.

The bubble rise-rate function is selected from theoretical models from (Woolf and Thorpe, 1991; Woolf, 1993; Leifer and MacDonald, 2003; Leifer and Patro, 2002; Merlivat and Memery, 1983; Mendelson, 1967). These require parameters of physical water conditions and chemical constants of the gas (in our case methane).

Water temperature, salinity and density was acquired from local CTD profiles. Sound speed was acquired from the velocity profiler. Surface tension and viscosity were correlated with the rest of the values using relationship from Miyake and Koizumi (1948). Theoretical research was used to obtain gaseous methane constants for specific heat 
capacity (Din, 1961; Rueff et al., 1988), specific heat ratio (Din, 1961), thermal conductivity (Prasad et al., 1984) and gas density at the surface (from underway methane recordings).

The flux $\left(\psi_{M}\right)$ of undissolved gas released from a seafloor opening is then calculated using Eq. 2.26.

$$
\psi_{\mathrm{M}}=\rho_{\mathrm{G}} 10^{\frac{\mathrm{TS}}{10}} \Psi
$$

\subsection{Error analysis}

\subsubsection{Bubble distance from the camera}

The degree of uncertainty in the size and elevation of a camera-imaged bubble is most significantly dependant on the location of the bubble in relation to the camera. Bubblesize calculations thus far have operated on the assumption that bubbles rise in a plane (plane 1 from Figure 2.21) that laterally extends across the seafloor aperture (v). Any bubble deviation in this plane will have a negligible effect on the precision of size calculations, as they can be observed in the video footage. Conversely, movement in and out of the plane will directly influence the position of the bubble along the line wW and hence affect the precision of both size and elevation calculations. Bubble displacements towards and away from the camera cannot be observed in the image plane, and are specific to each bubble in a seep plume. In order to estimate the error values for all bubbles in a still frame, the scripts offcentre.m and uncertainty.m (provided in Section $6.10 \& 6.11$ ) were used.

As a first approximation of out-of-view bubble movement, the assumption was made that: for any oblique scene distance X (Figure 2.21) that a bubble is observed to have migrated away from a vertical rise path, it is equally likely (for water of homogenous conditions) for that bubble to have travelled up to the equivalent distance in either perpendicular direction in the horizontal plane. This assumption is viable for deeper water where surface currents have a negligible effect. Measurements made by Gargett (1989), Drennan et al. (1992) and Anis and Moum (1992) indicate that surface-wave generated energy decays inversely to a power of 3 - 4.6. This decay implies that under- 


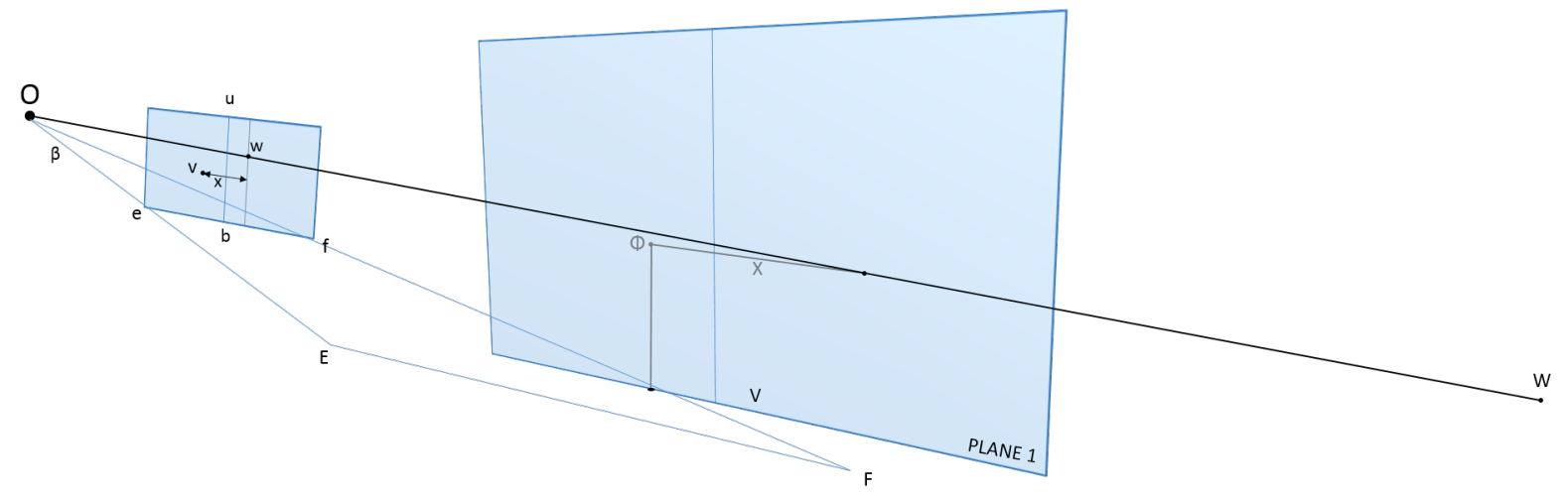

Figure 2.21: Geometry of the camera origin, image plane and plane 1, which extends vertically upwards from the seafloor aperture (V) and laterally parallel to EF. Bubblesize calculations are computed under the assumption that rising bubbles remain in plane 1. The line wW extends from the origin $(\mathrm{O})$, through the bubble $(\Phi)$ until it intercepts the seafloor. In this example the bubble has deviated a horizontal distance $\mathrm{X}$ from the zenith to the vent whilst rising.

currents and turbulent forcing generated by wind-driven waves and surface currents are depleted within the upper $100 \mathrm{~m}$ of the water column (Craig and Banner, 1994). Depth ranges concerning seeps along the Hikurangi Margin are therefore relatively unaffected by these influences.

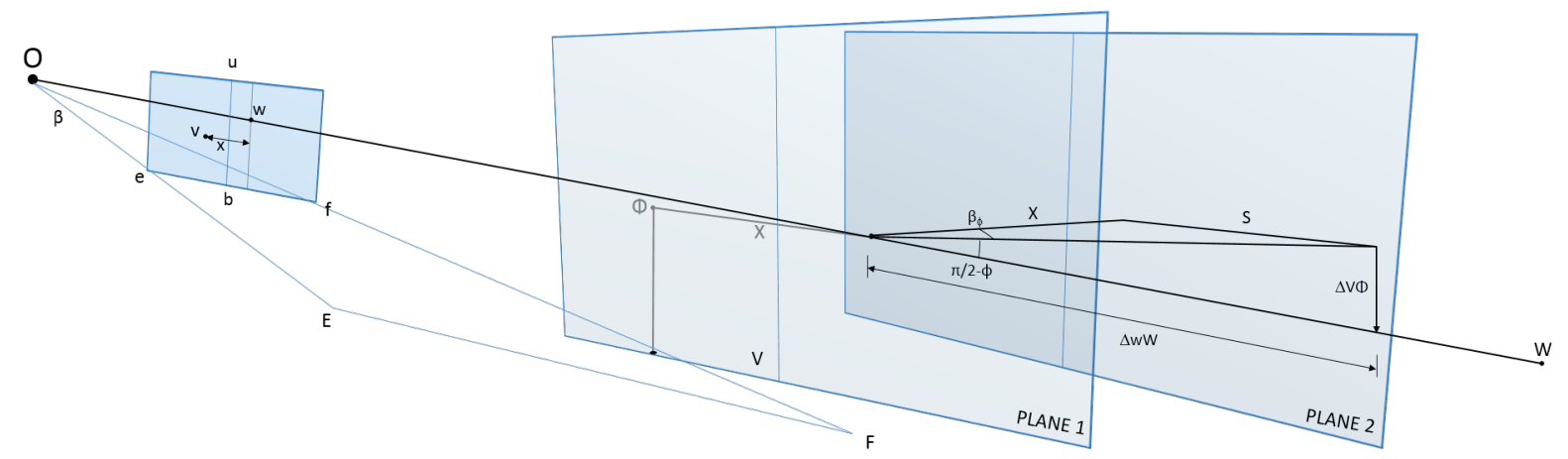

Figure 2.22: A second plane parallel to plane 1 (plane 2) has been added at a distance $\mathrm{X}$ away from plane 1 . This is the assumed possible distance that the bubble could have strayed away from the camera. Additional triangles display how this deviation effects size and height calculations.

The resultant range of ambiguity in the bubbles position is calculated from the geometry of Figure 2.22, using the following methods:

First, the true distance $X$ that the bubble has strayed horizontally in the plane is calculated. Distances are converted from image dimensions to scene dimensions using the same methods as for bubble radius. Once $\mathrm{X}$ has been evaluated for each bubble, this distance is assumed to be the maximum likely horizontal distance that the bubble 
has deviated either towards or away from the camera (Figure 2.22). The deviation along the line $\mathrm{wW}$ is then evaluated as $\pm \omega$ in Eq. 2.27.

$$
\pm \omega=\frac{S}{\cos \left(\frac{p i}{2}-\phi\right)}
$$

Here, $\mathrm{S}$ is calculated from Eq. 2.28.

$$
S=\frac{X}{\cos \left(\beta_{\phi}\right)}
$$

Maximum and minimum bubble radii uncertainties are then calculated by adapting Eq. 2.20 to give Eq. 2.29

$$
r_{n}\left( \pm \Delta r_{n}\right)=a_{n}+\left[\left(A_{n}-a_{n}\right) \cdot \frac{\overline{w W}-(\overline{W \Phi}+ \pm \omega)}{\overline{w W}}\right]
$$

The uncertainty in bubble height is equated using Eq. 2.30.

$$
\Delta V \Phi=S \tan \left(\frac{\pi}{2}-\phi\right)
$$

It can be seen from Figure 2.23 that the further the bubble is vertically from the camera, the larger the error will be associated with the bubble.

\subsubsection{Sinusoidal variations in bubble size measurements}

Preliminary measurement of bubble-sizes that we have conducted for this study have displayed sinusoidal variations in bubble radius as they rise. As it is unexpected for bubbles to noticeably change size over the first few metres of their ascent (McGinnis et al., 2006), we have assumed that these bubble-size variations are erroneous. To account for uncertainties created by sinusoidal variations, the peak amplitude of these bubble-radius deviations are added to the uncertainty of bubble-size calculations in the 'uncertainty' code (provided in Section 6.11). To better constrain this uncertainty, we have tracked and measured multiple bubbles over a succession of frames. We observed sinusoidal deviations in bubble radii of $\sim 1 \times 10^{-3} \mathrm{~m}$, which has been incorporated into bubble-size uncertainty for all of our calculations. These size-deviation errors can 


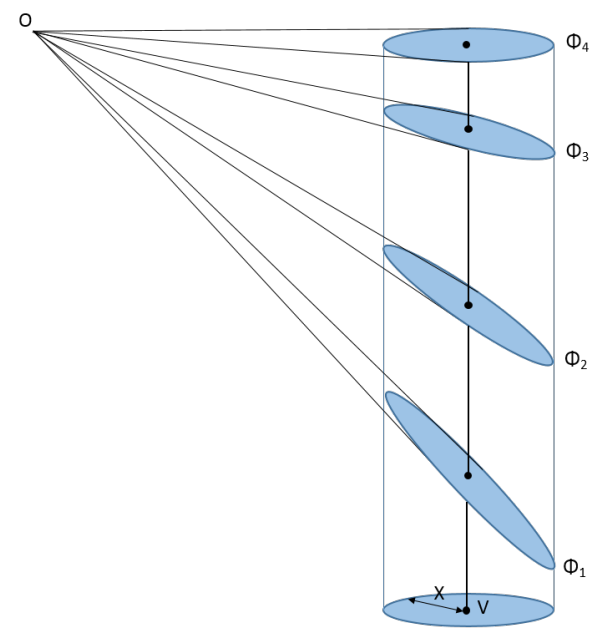

Figure 2.23: A cylinder of radius $\mathrm{X}$ has been drawn around the vent $\mathrm{V}$, illustrating the scope of the distance that a bubble is likely to deviate under the stated assumptions. Four ellipses have been drawn with the same deviation distance $\mathrm{X}$ at different vertical distances from the camera at $\mathrm{O}$. As the vertical difference increases so does the length of the major axis of the deviation ellipse. This results in larger errors associated with bubbles vertically further from the camera.

be adjusted by the user depending on the amount of variation that is observed in preliminary bubble-size measurements.

\subsubsection{Unquantified sources of error}

Other errors associated with the bubble-size calculation method that were deemed negligible in comparison to the considered error sources include: angled profiles of each bubble to the camera and the pixelated boundaries of bubbles apparent when picking major and minor axes.

Spheroidal bubbles ascend in a direction parallel to the axis of symmetry ( $c$ in Figure 2.24) (Ellingsen and Risso, 2001). Viewing bubbles at an inclined angle rather than directly in-line with the camera will result in a slightly larger perceived short axis $\left(c^{\prime}\right.$ in Figure 2.24), which also results in an larger observed equivalent-spherical bubble radius.

The resolution of the video-recorded still frames also begins to deteriorate when enlarged enough to closely analyse bubble axes. Low-resolution imagery creates difficulties in precisely defining the ends of the major and minor axes along the boundary edges. Uncertainties that arise from low resolution imagery can either increase or 
A

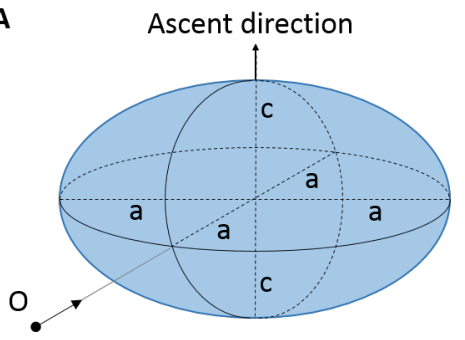

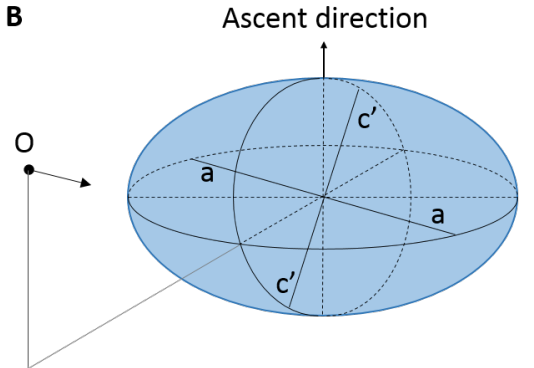

Figure 2.24: Observed dimensions of oblate-spheroidal bubbles by a camera positioned at $\mathrm{O}$ oriented in the arrow direction. The major and minor axes of the oblate-spheroidal are labelled a and c respectively. In the case of $[\mathrm{A}]$, the camera is observing the bubble in the plane of the major axis. From this angle, the true dimensions of a and $\mathrm{c}$ are measurable. Conversely, the camera in [B] is positioned above the bubble, facing downwards. The dimension of a is still true; however the observed short axis length c' is larger than $\mathrm{c}$.

decrease the outputted equivalent-spherical bubble radius.

Both errors in this section were not considered in calculations as they contribute negligible error to final methane-flux measurements in comparison to previously discussed bubble deviations and sinusoidal size variations. 


\section{Results}

We have applied the seep analysis techniques (detailed in the Chapter 2) to the flares situated in the $50 \mathrm{~km}^{2}$ section of the Hikurangi Margin (see Figure 3.1 \& 3.2). EM302 Multibeam data collected over TAN1404 and TAN1505 have been used to investigate flares over a regional (covering the $50 \mathrm{~km}^{2}$ area) and localised $\left(<0.1 \mathrm{~km}^{2}\right)$ scale. To assess the location and number of seeps in the survey region, multibeam data from the two voyages have been processed using SonarScope to produce regional intensity maps that highlight flare locations. The same multibeam data set has then been used to study the vertical changes in acoustic intensity for single seeps at staggered water depths.

To estimate the flux of undissolved methane emitted from submarine seeps, we have applied the photogrammetry codes (provided in Section 6.3 - 6.11) to measure the size and rise-rates of bubbles captured in underwater video footage collected on TAN1505. This footage has then been combined with singlebeam flare profiles to calculate the flux of undissolved methane at the base of seeps using the FlareFlow Module.

\subsection{Multibeam data}

Watercolumn imagery from the collected multibeam data identified multiple seeps based on evidence of sonar echoes from rising bubbles. We have further utilised these acoustic data to study the intensity of seeps by vertically summing the amplitudes of these echoes. This is useful over both a regional scale for assessing the spatial distribution of seeps, as well as a local scale for studying preservation of seep intensity at shallowing depths.

\subsubsection{Regional seep distribution and total seep count}

The combined capabilities of SonarScope and ArcMap have been used to acquire the location of seeps surveyed on TAN1404 and TAN1505. This information is useful for (1) counting the total number of surveyed seeps, (2) assessing areas where seeps are most concentrated, and (3) identifying relationships between seep location and bathymetric features such as carbonate mounds. 
We have used SonarScope to vertically sum the water-column echoes collected over both voyages using a EM302 Multibeam Echosounder. TAN1404 coverage has been divided into five areas (Figure 3.1), while the TAN1505 voyage has been divided into six smaller areas. The coverage of the TAN1505 voyage has been divided into six smaller areas where a dense population of seeps were expected to exist, based on findings from the TAN1404 voyage. Summed intensity maps produced using SonarScope (see Figure 3.3) display zones of relatively-high summed intensities, indicative of acoustically imaged seeps. These zones allow mapped seeps to be characterized by summed-reflective intensity values above a certain threshold. By adjusting the intensity-threshold range and comparing summed-intensity surfaces to horizontally stacked curtains (as in Figure 1.1), we have chosen threshold values that accurately represent the lower limit of identified flare features. Maximum and minimum threshold values have been chosen for each area in Figure $3.1 \&$ 3.2, that best limit the summed intensity surfaces to only include flare echoes.

We have used these threshold values as a means to estimate the number of seeps in each region using tools in ArcMap. By reclassifying each surface to values above the theshold values for each surface, we have filtered the data so that only seep indicators remain. The maximum and minimum number of these features in each region have been tabulated in Table 3.1. 


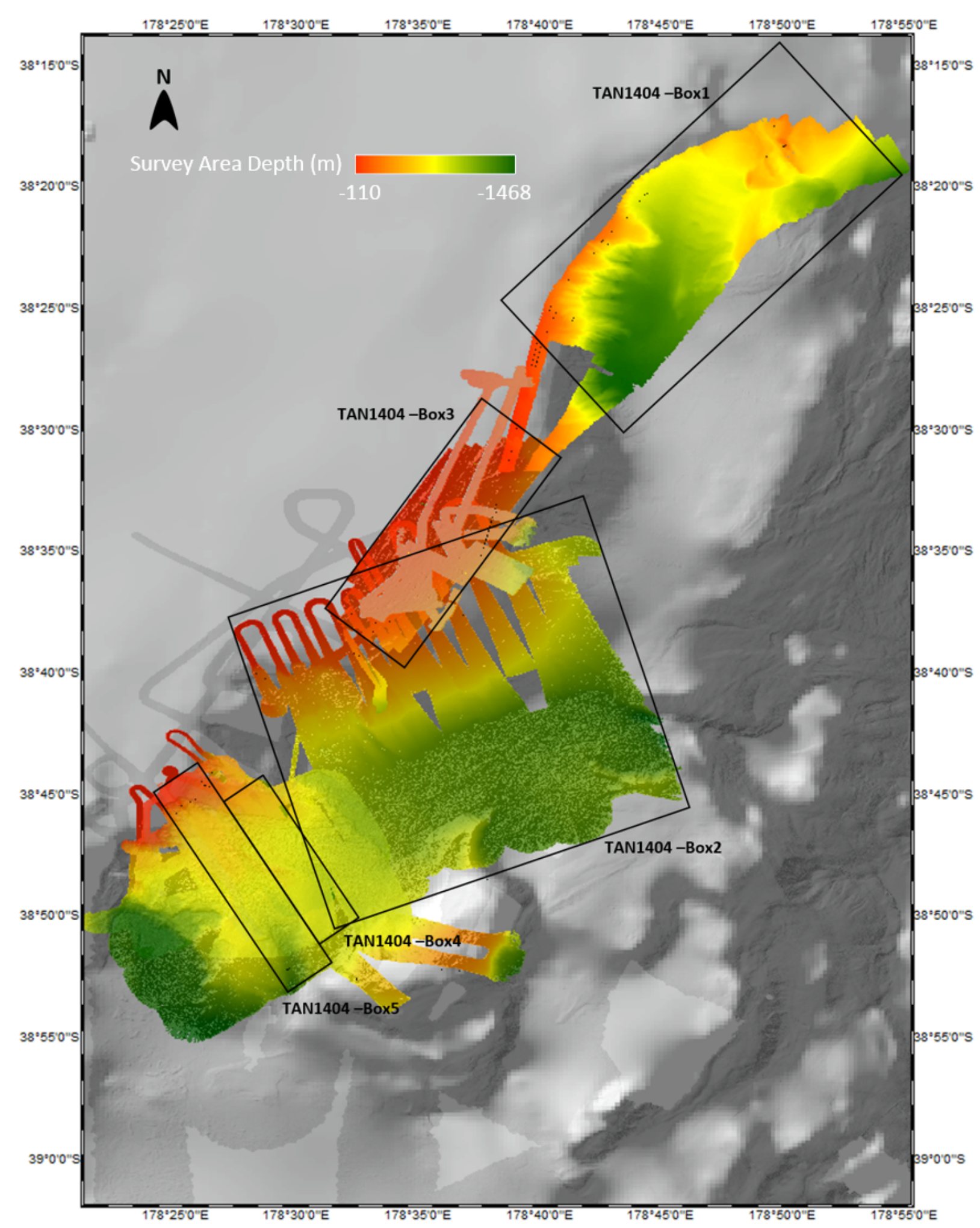

Figure 3.1: Multibeam coverage over the part of the upper-rise of the Hikurangi Margin from TAN1404 and TAN1505. For the purpose of producing summed intensity maps, the TAN1404 coverage has been divided into the 5 different areas shown here. 


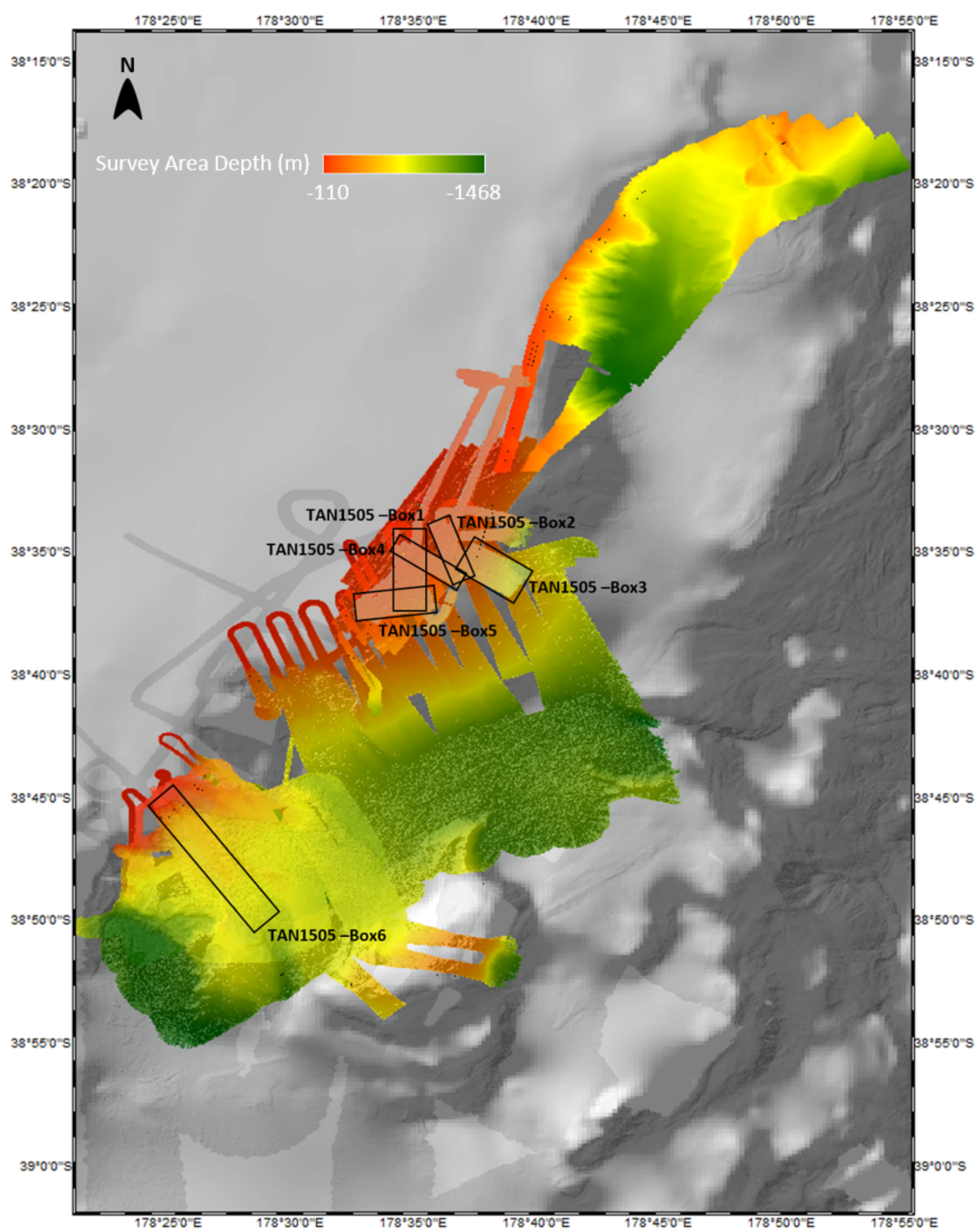

Figure 3.2: Multibeam coverage over part of the upper-rise of the Hikurangi Margin Bathymetry from TAN1404 and TAN1505. For the purpose of producing summed intensity maps, the TAN1505 coverage has been divided into the 6 different areas shown here. 

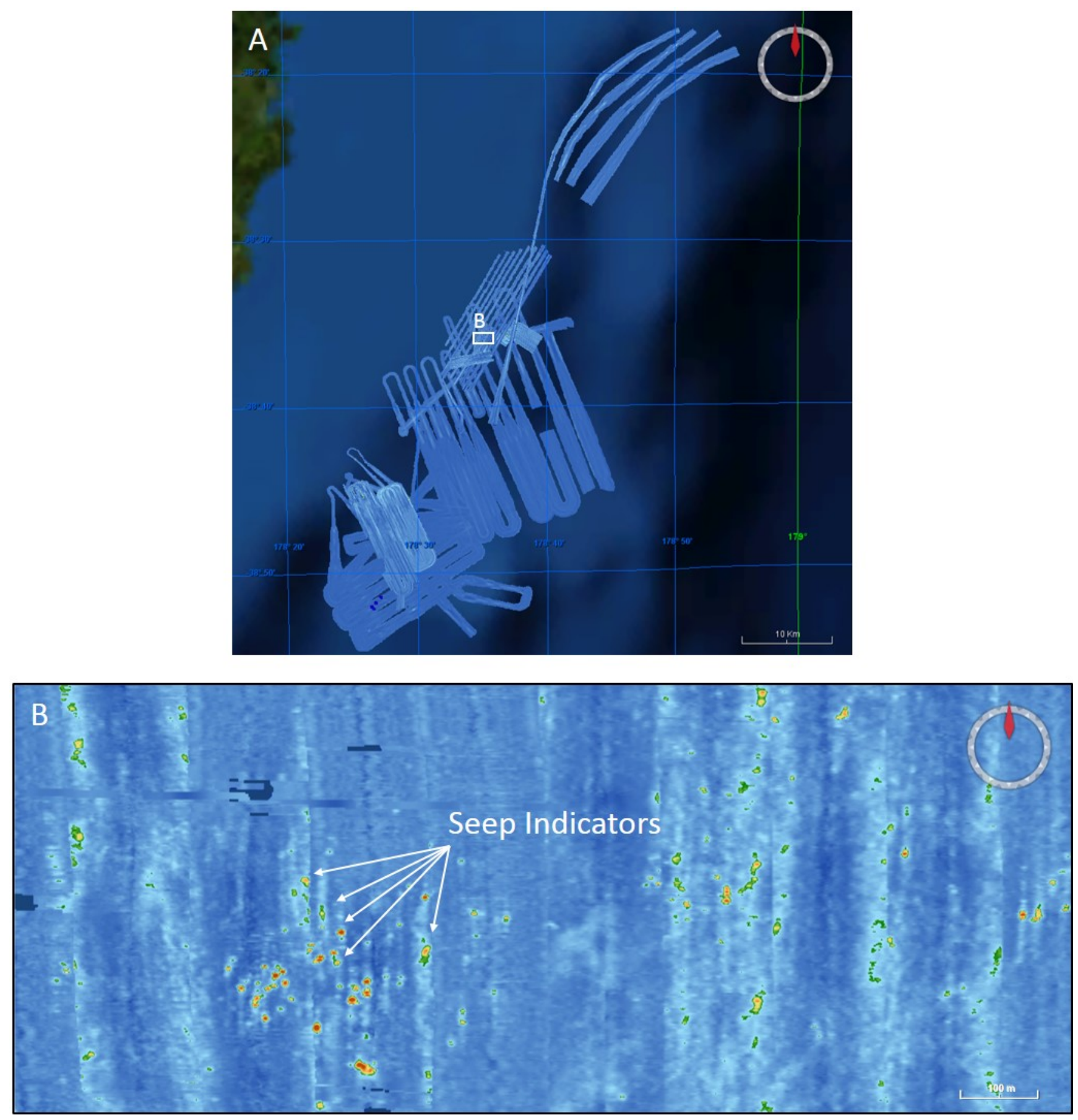

Figure 3.3: [A] Summed intensity maps for the multibeam coverage of TAN1404 and TAN1505. [B] Zoomed section that displays examples of high-amplitude zones indicative of seep-bubble plumes. 


\begin{tabular}{|l|l|l|}
\hline & \multicolumn{2}{|c|}{ Estimated Number of Seeps } \\
\hline Region & Minimum & Maximum \\
\hline TAN1404-Box1 & 20 & 22 \\
TAN1404-Box2 & 338 & 382 \\
TAN1404-Box3 & 178 & 206 \\
TAN1404-Box4 & 8 & 8 \\
TAN1404-Box5 & 39 & 44 \\
\hline TAN1404 Total & 585 & 660 \\
\hline TAN1505-Box1 & 90 & 102 \\
TAN1505-Box2 & 13 & 15 \\
TAN1505-Box3 & 7 & 8 \\
TAN1505-Box4 & 80 & 94 \\
TAN1505-Box5 & 35 & 39 \\
TAN1505-Box6 & 11 & 13 \\
\hline TAN1505 Total & 236 & 271 \\
\hline
\end{tabular}

Table 3.1: Tabulated minimum and maximum estimates for the number of seeps identified in each region from Figure $3.1 \& 3.2$. 


\subsection{Seep-bubble preservation at staggered depths}

We have applied the OpendTect vertical summation method to an exemplary flare on line 0216 (Figure 3.4) from the TAN1404 voyage. This allowed us to observe the preservation of flare intensity with increasing elevation above the seafloor. Summedintensity surfaces (Figure 3.6) were calculated between horizontal surfaces (area shown in Figure 3.5) from the seafloor (at $0 \mathrm{~m}$ elevation) to $170 \mathrm{~m}$ above the seafloor in $10 \mathrm{~m}$ increments to cover the $164 \mathrm{~m}$ watercolumn.

To quantify the change in summed-intensity with increasing elevation, histograms of the summed-intensity values across the area of each surface have been plotted in Figure 3.7. Summed intensity values for each surface in Figure 3.6 have been binned using the histogram algorithm in Matlab. This function separates the data into bins of uniform width, chosen to cover the range of summed intensity and reveal the underlying shape of the distribution. Histograms have been plotted for each $10 \mathrm{~m}$ elevation increment, ordered by decreasing decibel values of summed intensity.

In this case more negative values of summed intensity indicate raised seep activity. Decibels are a measure of the ratio of energy emitted from a source (in our case a multibeam echosounder) to received energy that has been echoed from a body (in our case bubbles). Echoes from bubbles are weaker than the emitted multibeam signal, so all acoustically imaged bubbles are represented in negative decibel values. When these bubbles intensity values are vertically summed, a higher number of bubbles will amount to a more negative total summed intensity value.

The plot shows distinct peaks at approximately the same summed amplitude value $(\sim$ $-200 \mathrm{~dB}$ ). These peaks are likely due to natural amplitude responses of a collection of bubbles in the $10 \mathrm{~m}$ elevation windows. The decibel values of both peaks have remained relatively constant throughout the extent of the water column. The occurrence of these 'bubble-frequency' responses has decreased (apparent in Figure 3.7) at higher elevations above the seafloor, likely due to a decreasing number of bubbles.

Data trends in Figure 3.7 are difficult to interpret due to their complexity. In order to better constrain the trends in the data, a second histogram plot has been generated for a cropped area (displayed in Figure 3.8) so that summed-intensity values exclusive to bubbles from the single, central seep (Flare 1 in Figure 3.6) can be analysed. The histograms displayed in Figure 3.9 are binned using the same Matlab algorithm as in Figure 3.7, revealing similar trends but less contribution of reflections of $\sim-200 \mathrm{~dB}$ 
and a higher percentage around $\sim-1500 \mathrm{~dB}$.

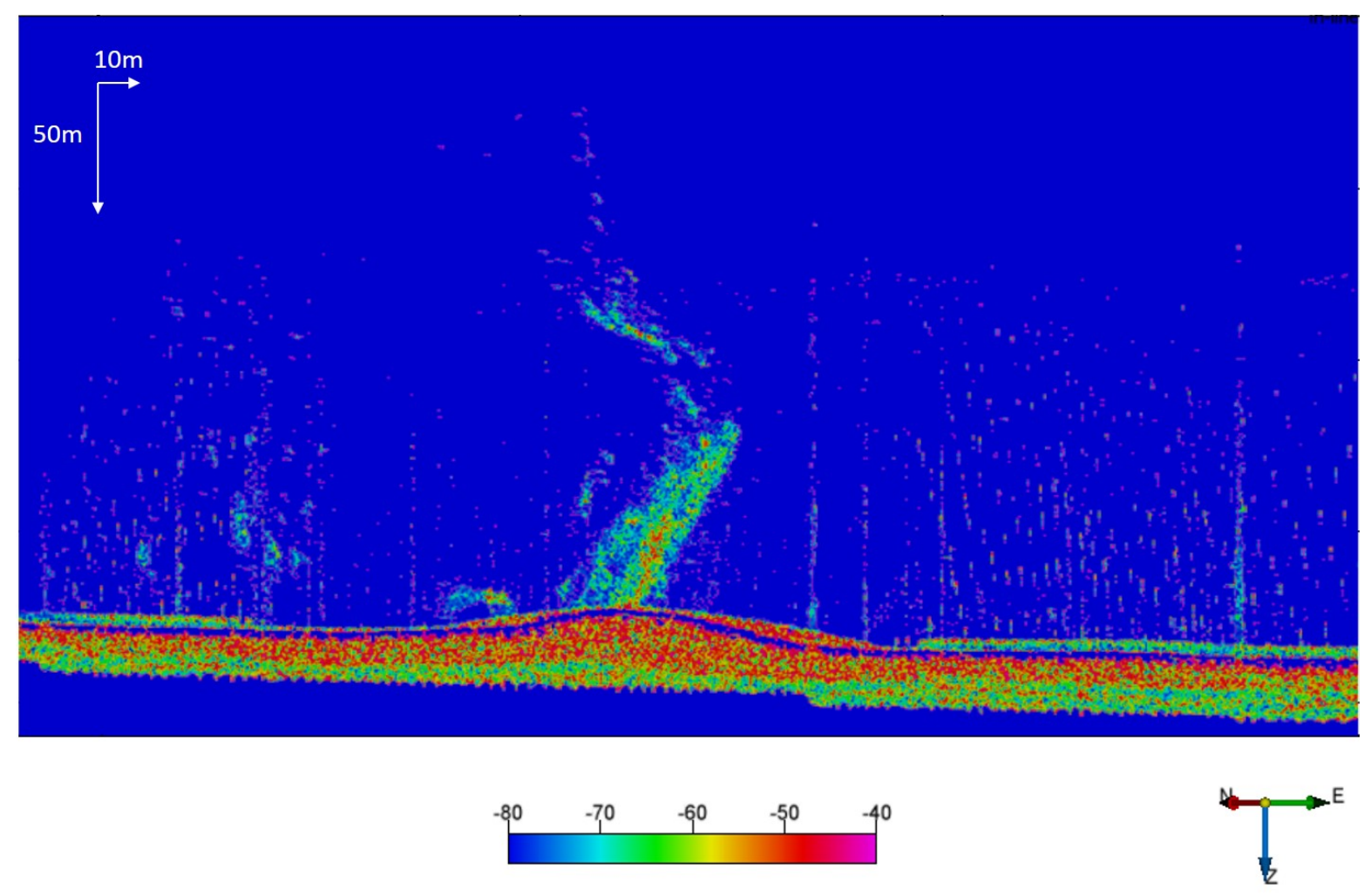

Figure 3.4: Along-track face of a voxet that covers part of line 0216 containing the analysed flare. This flare has particularly good coverage at all parts of the bubble assent with minimal noise disruption from proximal biology and ocean-current effects. 


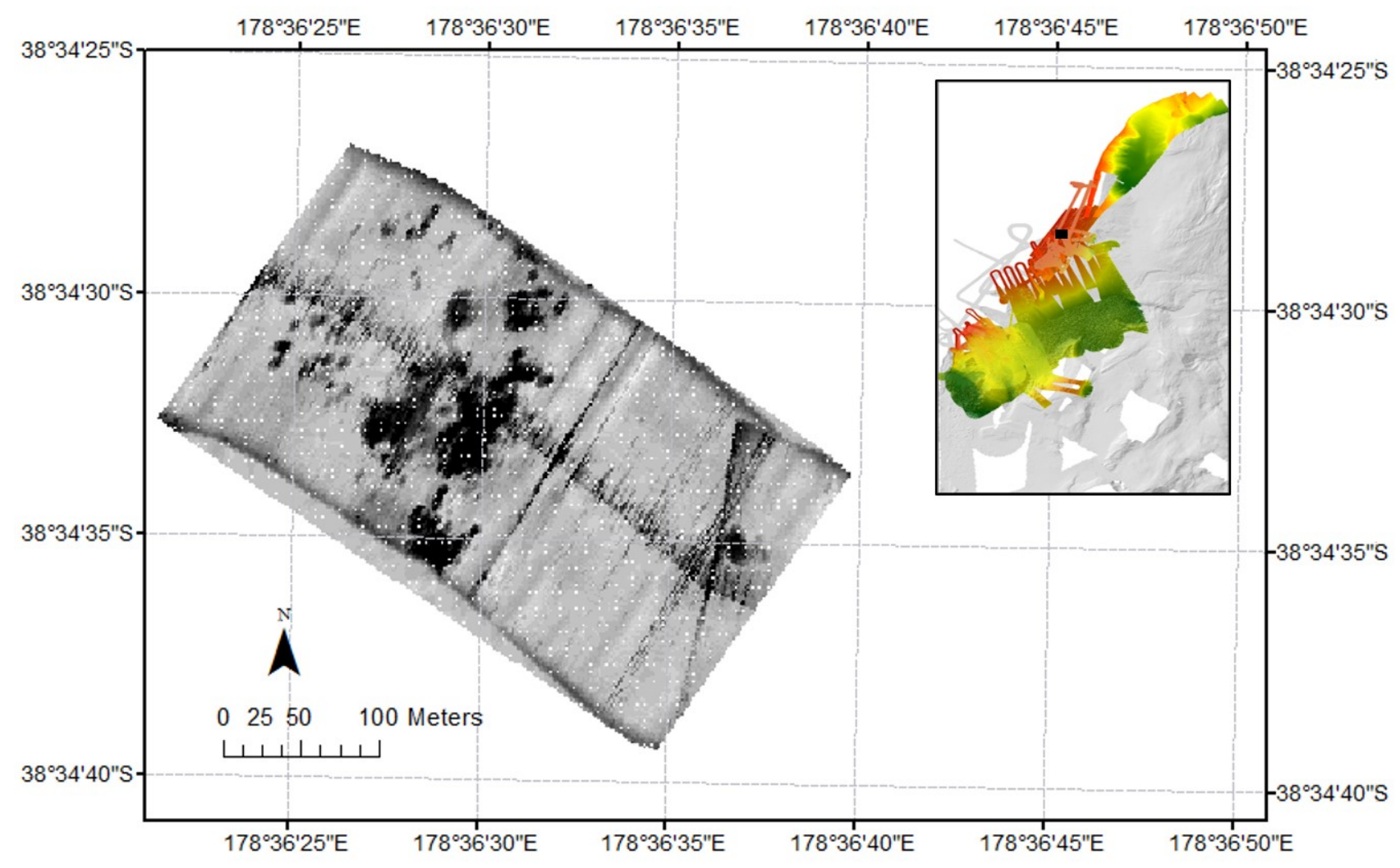

Figure 3.5: Horizontal bounds of the summed intensity surfaces (Figure 3.6) that have been used to study varying seep intensity. The location of the area in the survey region is also displayed (black square) in map-view. 
$0 m$ to $10 m$

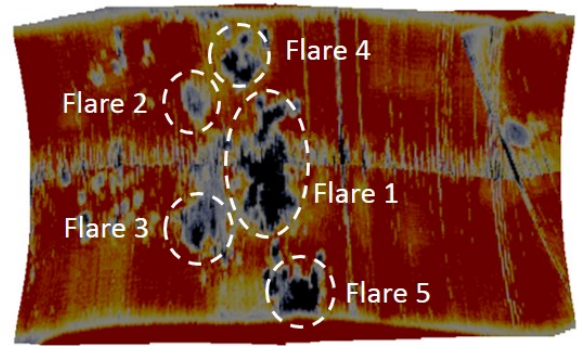

$50 \mathrm{~m}$

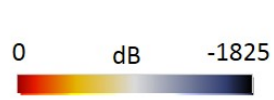

$20 \mathrm{~m}$ to $30 \mathrm{~m}$
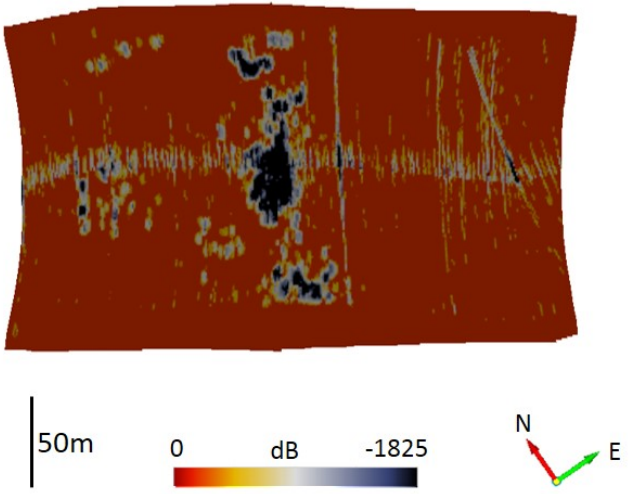

$40 m$ to $50 m$
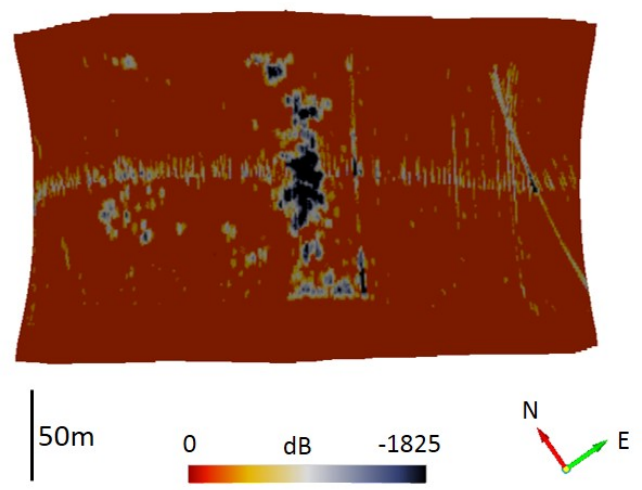

$60 m$ to $70 m$
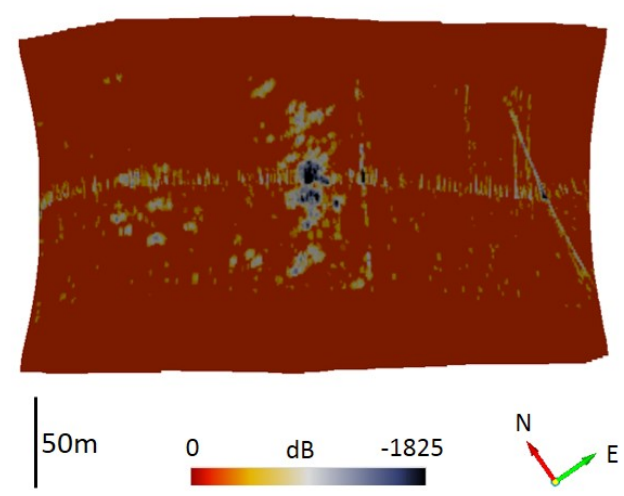

$10 \mathrm{~m}$ to $20 \mathrm{~m}$
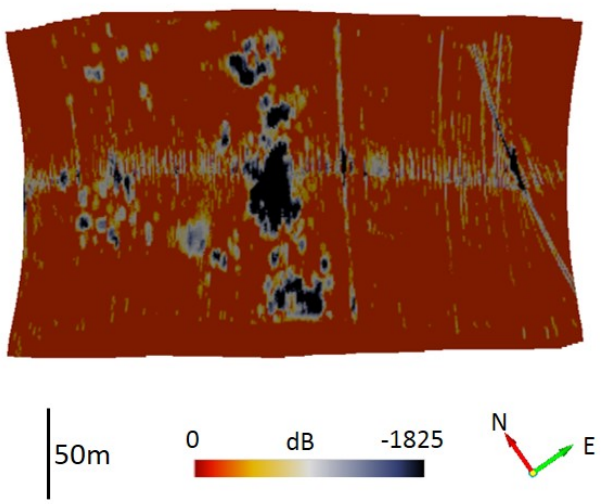

$30 m$ to $40 m$
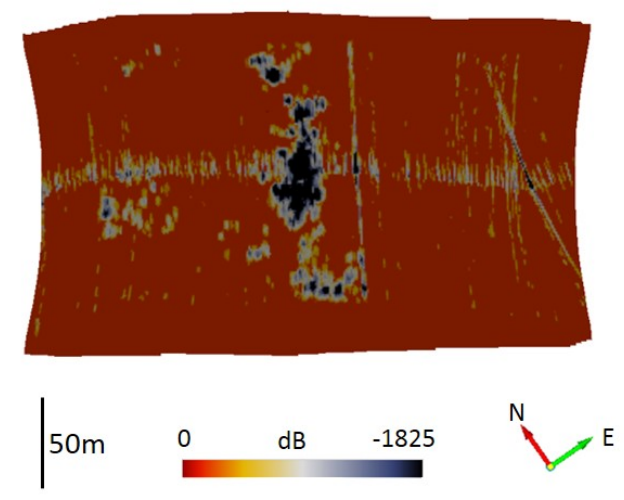

\section{$50 \mathrm{~m}$ to $60 \mathrm{~m}$}
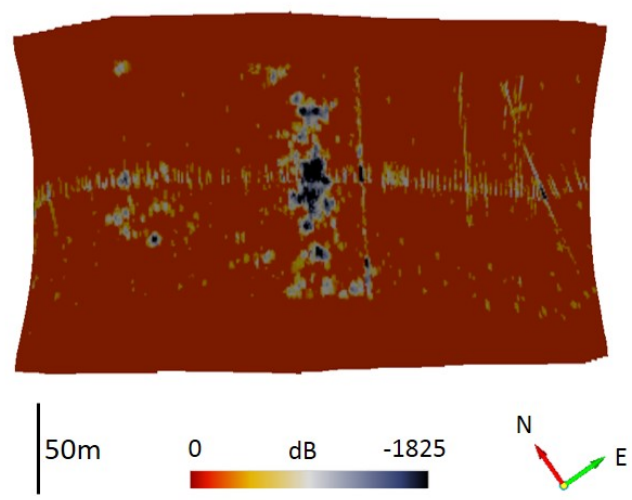

$70 \mathrm{~m}$ to $80 \mathrm{~m}$
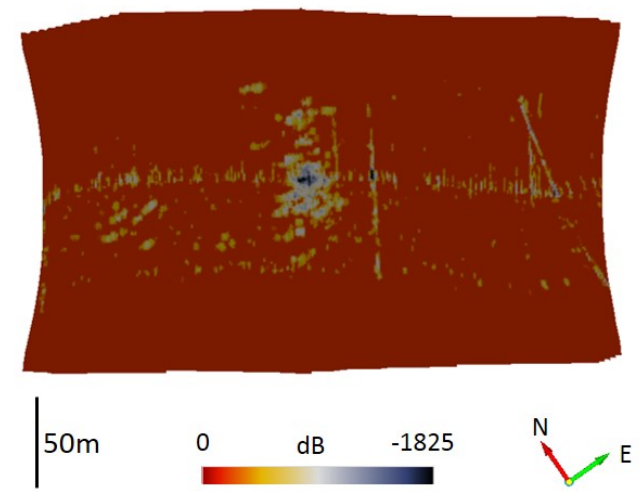
$80 m$ to $90 m$
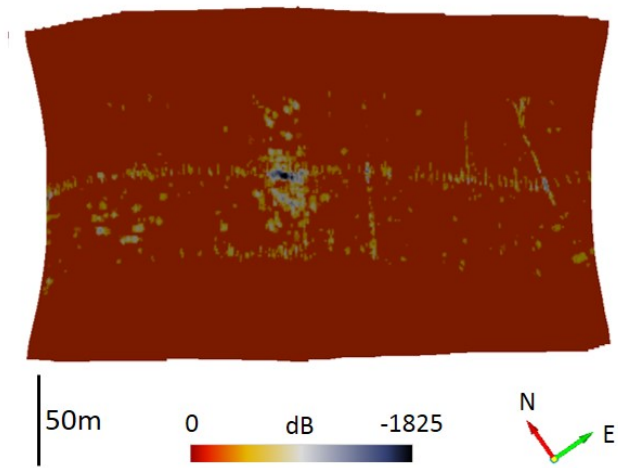

$100 \mathrm{~m}$ to $110 \mathrm{~m}$

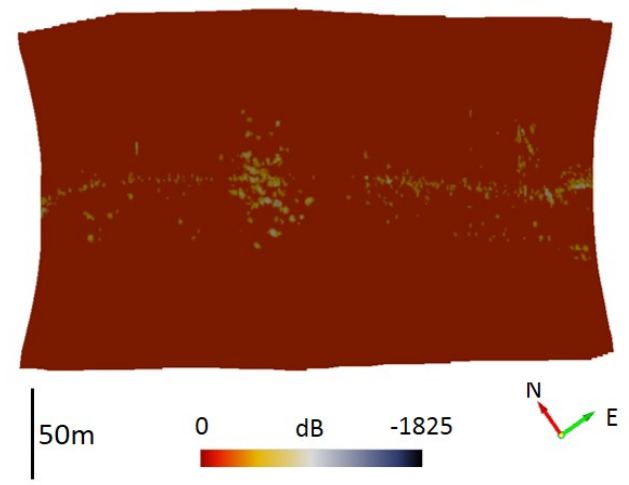

$120 \mathrm{~m}$ to $130 \mathrm{~m}$

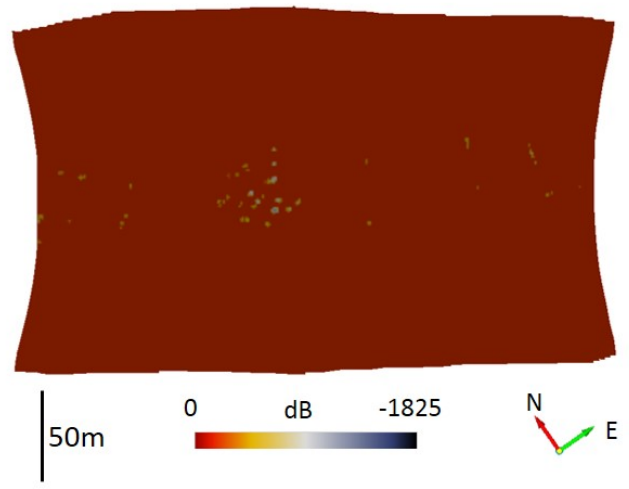

$140 \mathrm{~m}$ to $150 \mathrm{~m}$

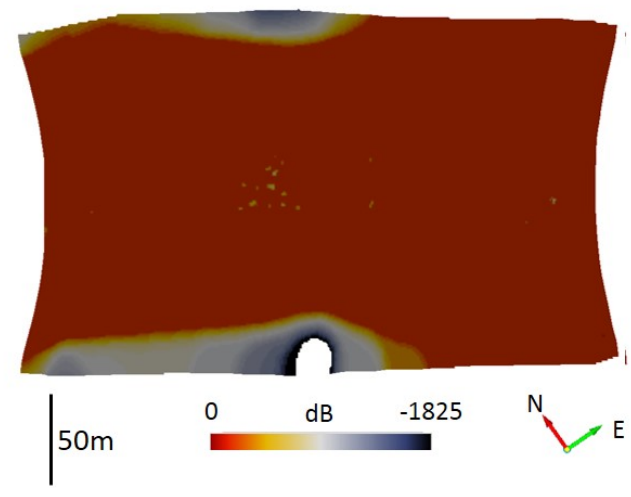

$90 \mathrm{~m}$ to $100 \mathrm{~m}$
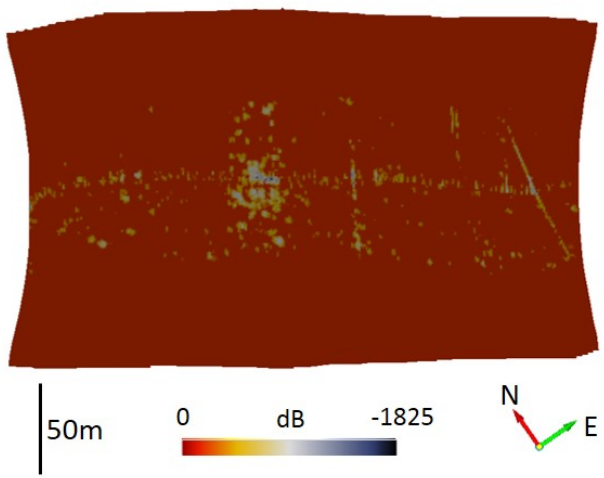

$110 \mathrm{~m}$ to $120 \mathrm{~m}$

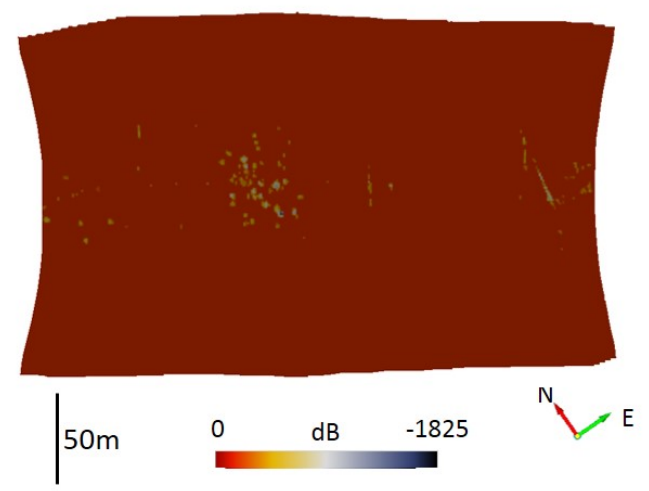

$130 \mathrm{~m}$ to $140 \mathrm{~m}$

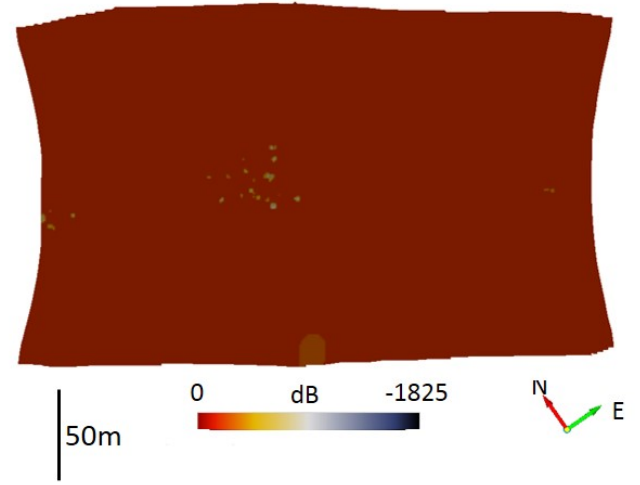

$150 \mathrm{~m}$ to $160 \mathrm{~m}$

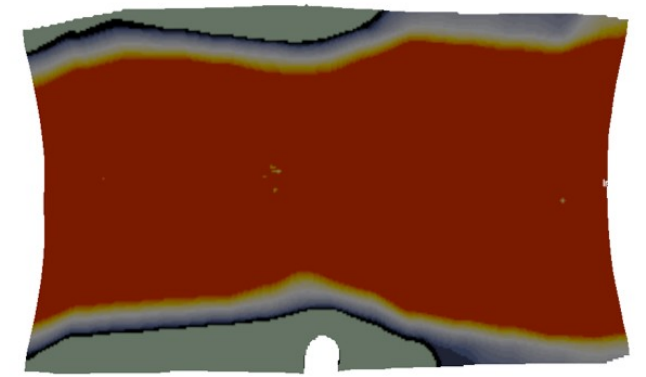

$\mid 50 \mathrm{~m} \quad \mathrm{O} \quad \mathrm{dB} \quad-1825 \quad \mathrm{~N}$ 


\section{$160 m$ to $170 m$}

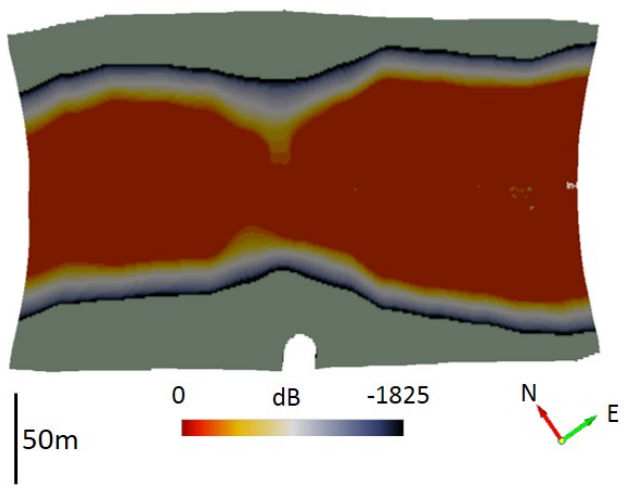

Figure 3.6: Summed surfaces for $10 \mathrm{~m}$ elevation bins for a flare on line 0216 of the TAN1404 voyage displayed in Figure 3.4. The distance labels are measured from the seafloor at the base of the flare. Each surface is coloured by summed echoed intensity $(\mathrm{dB})$. More negative values indicate raised seep activity.

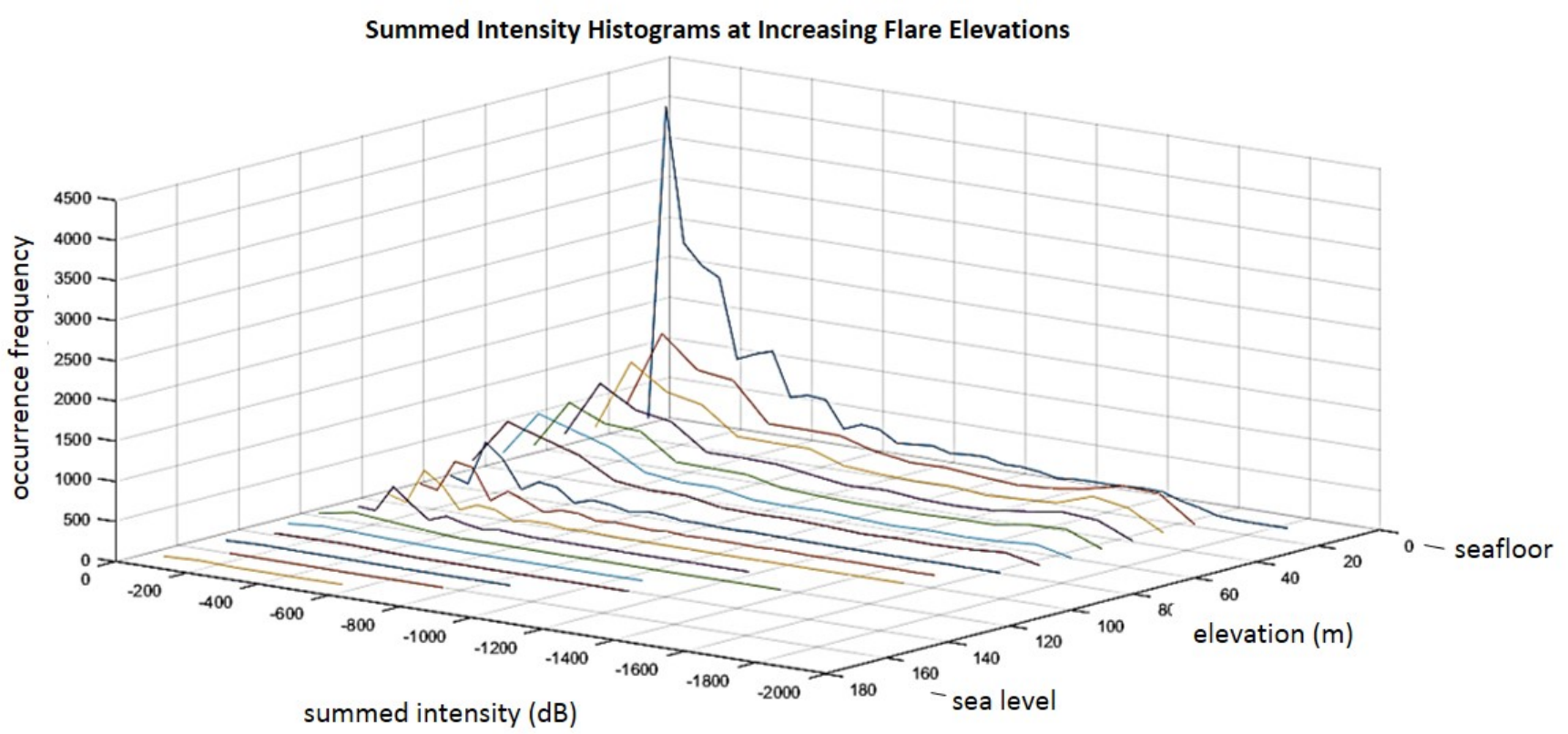

Figure 3.7: Histograms for each surface displayed in Figure 3.4, each plot has been positioned at the upper-most depth of the summed-intensity range for each surface. 


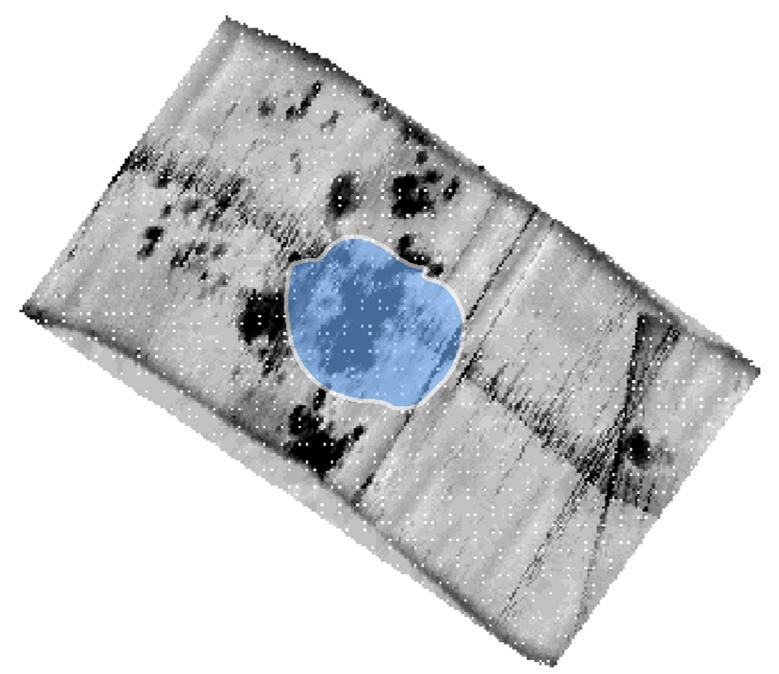

Figure 3.8: Cropped area of the flare under further investigation for summed-intensity changes. The orientation and scale of the surface is the same as for Figure 3.5.

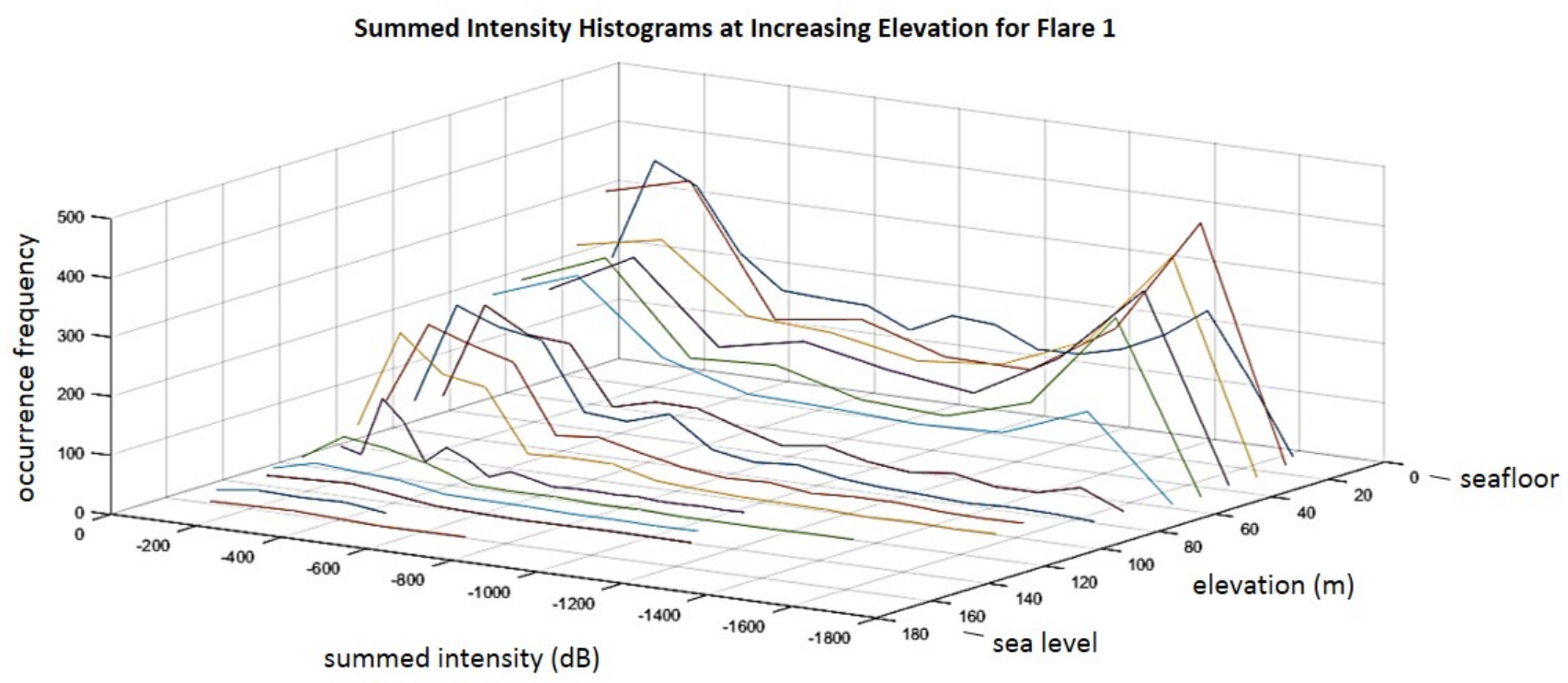

Figure 3.9: Histograms have been generated for summed surfaces in Figure 3.4, cropped to the area shown in Figure 3.8. Each plot has been positioned at the upper-most depth of the summed-intensity range for each surface. 


\subsection{Bubble size and rise-rate measurements}

\subsubsection{Bubble radius distribution}

The equivalent spherical radii of emitted seep bubbles recorded using DTIS on TAN1505 were calculated using the Matlab codes for perspective measurements (provided in Section 6.3 - 6.11). The chosen video recording captures approximately 90 seconds of seep activity which sees the discharge of collections of bubbles in four distinct bursts. For one of these bursts (lasting $\sim 9 \mathrm{~s}$ ) the camera was positioned just above the seafloor, allowing for a detailed view of bubbles at the initial stage of their ascent.

Bubble-size results were collected for frames where bubbles were clearly defined (plotted in Figure 3.10). Uncertainties in bubble-size measurements are directly proportional to the deviation of each bubble from a vertical rise path. This is evident from the degree of uncertainty in bubbles plotted in Figure 3.10, which increases with progressing frame numbers (i.e. increasing bubble elevation).

In order to acquire accurate bubble radius measurements, frames must be used that capture a collection of bubbles at a stage of their ascent that best represents the true bubble-size distribution. As bubbles are being released from the seafloor in bursts, early video frames will not capture a representative number of bubbles to correctly estimate the size distribution. Conversely, higher errors associated with higher rise-distances also make it difficult to constrain an accurate estimation of a bubble-size distribution in later frames. Of the frames that are plotted in Figure 3.10, frame 160 has been chosen as a time increment with a suitable range of bubble sizes with a reasonably low uncertainty.

Further bubble-size measurements have been made within five frames of frame 160 (Figure 3.11). To acquire distributions of bubble radius that are representative of the size of bubbles emitted from the seafloor. A 5 th order polynomial fit has been applied to the collated maximum and minimum size distributions from Figure 3.11 (plotted in Figure 3.12). These fits have been constrained to a value of 1 at the highest point of the collated measurements. 

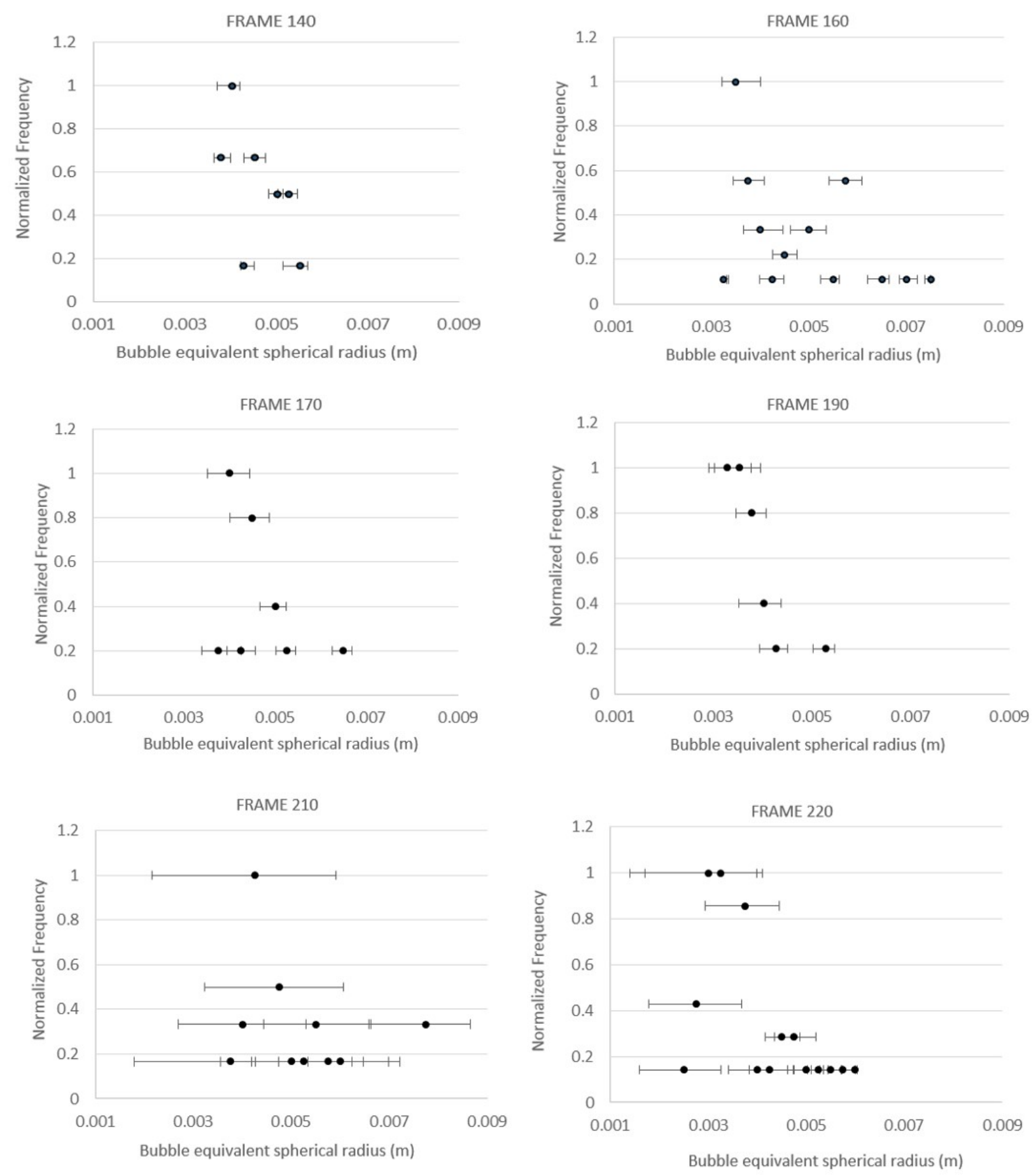

Figure 3.10: Bubble-size measurements from DTIS video frames (black) with minimum (red dotted) and maximum (blue dotted) uncertainty values. These frames cover $2.7 \mathrm{~s}$ for the same burst of ascending bubbles. 

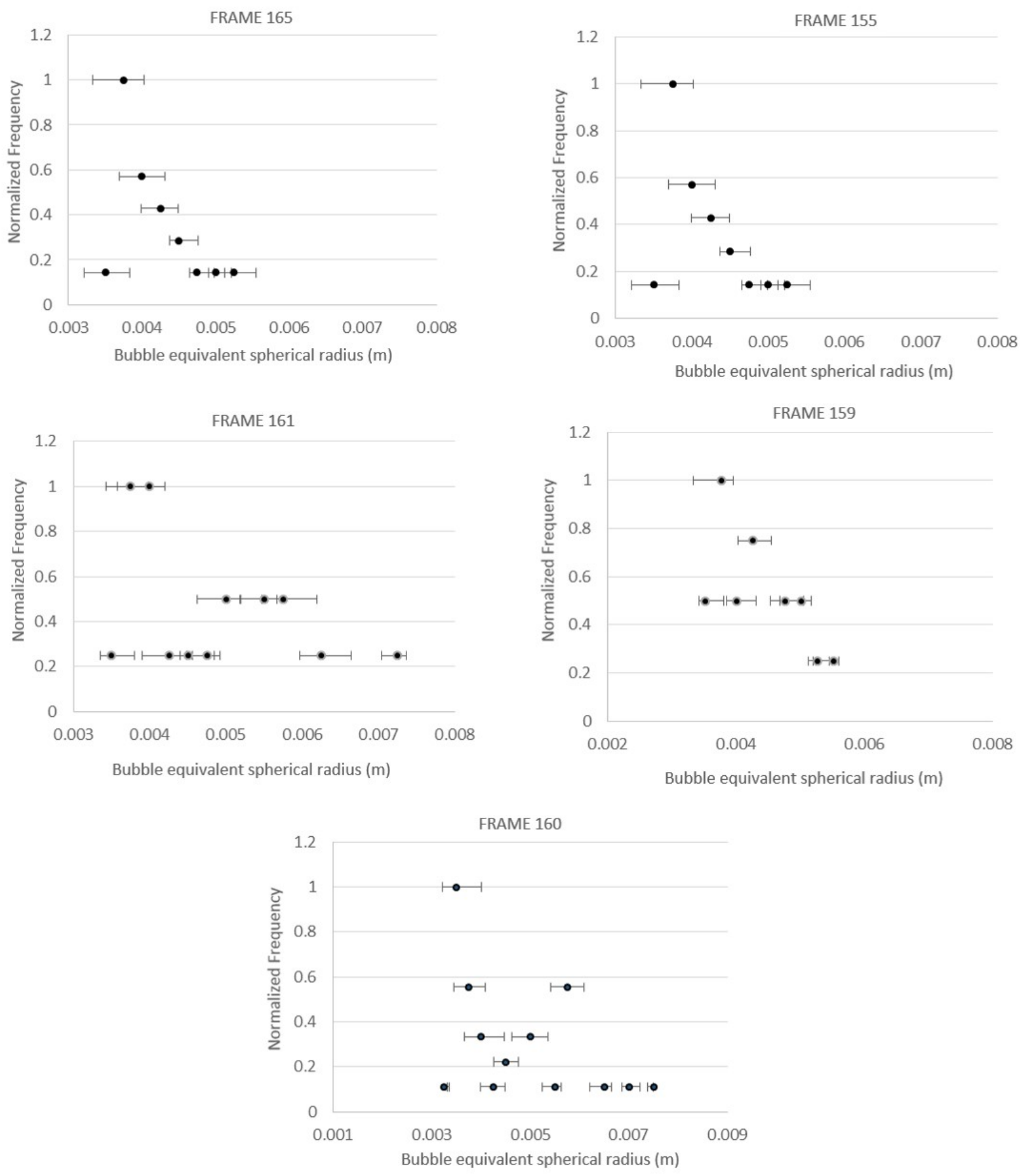

Figure 3.11: Bubbles-size measured over $0.3 \mathrm{~s}$ from DTIS video frames (black) with minimum (red dotted) and maximum (blue dotted) values. 


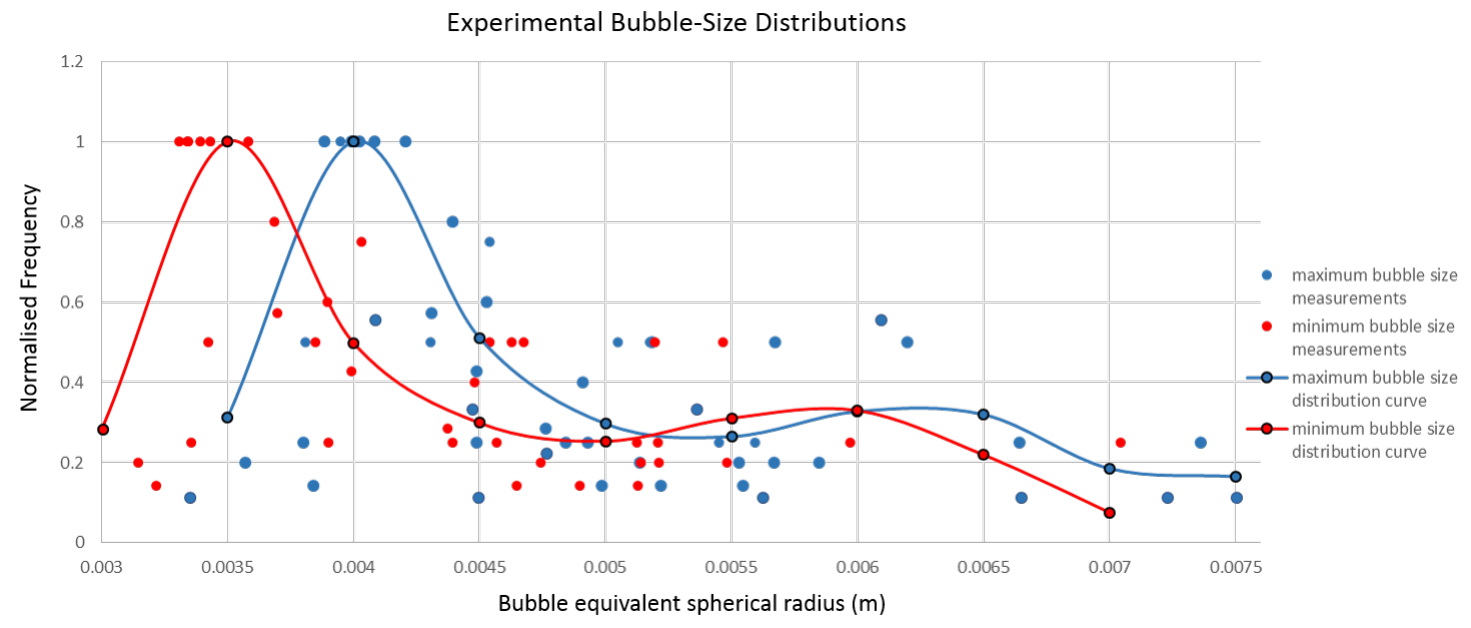

Figure 3.12: Representative minimum (red) and maximum (blue) bubble-size distribution curves. Both curves have been generated using a 5th order polynomial fit to the collated data from Figure 3.11 with a constrained value of 1 at the highest point. 


\section{Tracked Bubble Radius}

The same bubble-size measurement techniques have been applied to three bubbles (Sample bubbles 1, $2 \& 3$ ) that have been tracked through a series of video frames (see Figure 3.13). Here, there are two notable radius trends to observe in the time series of measurements. It can be seen from the distribution of the data that there are small scale fluctuations in bubble size with a period of $\sim 0.5 s$ as well as larger-scale gradients that differ across the entire time series and across each bubble.

Larger-scale radius trends (such as the negative overall trend of Sample Bubble 1 in Figure 3.11) are a result of bubbles drifting away from a vertical rise path. In the case of Sample Bubbles 2 and 3 (Figure 3.11), although there are superimposed small scale radius fluctuations, linear fitted trends reveal there are minimal long-term gradients. Negligible gradients suggest that these bubbles are rising sub-vertically from the seafloor. Conversely, Sample Bubble 1 is getting apparently smaller over time, indicating the bubble is drifting further from the camera as it rises.

To approximate the true size of each bubble, we have taken the y-intercept of each trend. This is likely an accurate approximation of the true size in the case of Bubble 1 and 3. In the case of Bubble 2, this will give the best estimate of the true size over the recorded time, as the bubble would have strayed a minimum amount.

\section{Bubble Rise Rates}

To evaluate the rate at which bubbles of certain radii rise, the elevation above the seafloor of Sample Bubble 1,2 and 3 were also recorded over a series of video frames. The elevations of Sample Bubble 1,2 and 3 are plotted in Figure 3.14 against the radius of the bubbles estimated from the linear trends (Figure 3.13). Linear trends are a suitable fit to all of the elevation time-series measurements, indicating the bubbles have risen at a constant speed over each period of observation. Linear trends have been fitted to the measured values as well as to the associated error calculations. The gradients of each trend have been used to determine rise-rates of $16.36-20.12 \mathrm{~cm} / \mathrm{s}$ for a bubble of radius $0.54 \mathrm{~cm}$ (Sample Bubble 1), $8.77-19.41 \mathrm{~cm} / \mathrm{s}$ for a bubble of radius $0.56 \mathrm{~cm}$ (Sample Bubble 2), 17.63-20.67 cm/s for a bubble of radius $0.39 \mathrm{~cm}$ (Sample Bubbble 3). The radius and velocity measurements of bubble 1, 2 and 3 have been plotted in Figure 3.15. 


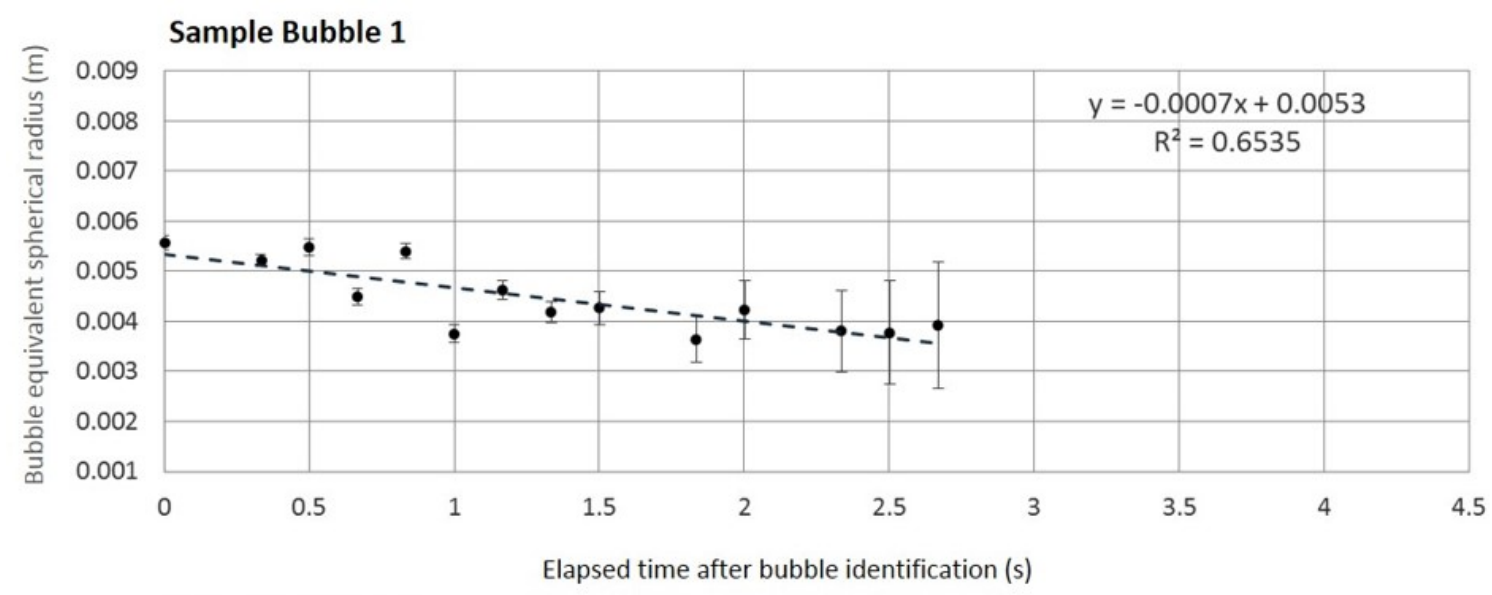

Sample Bubble 2
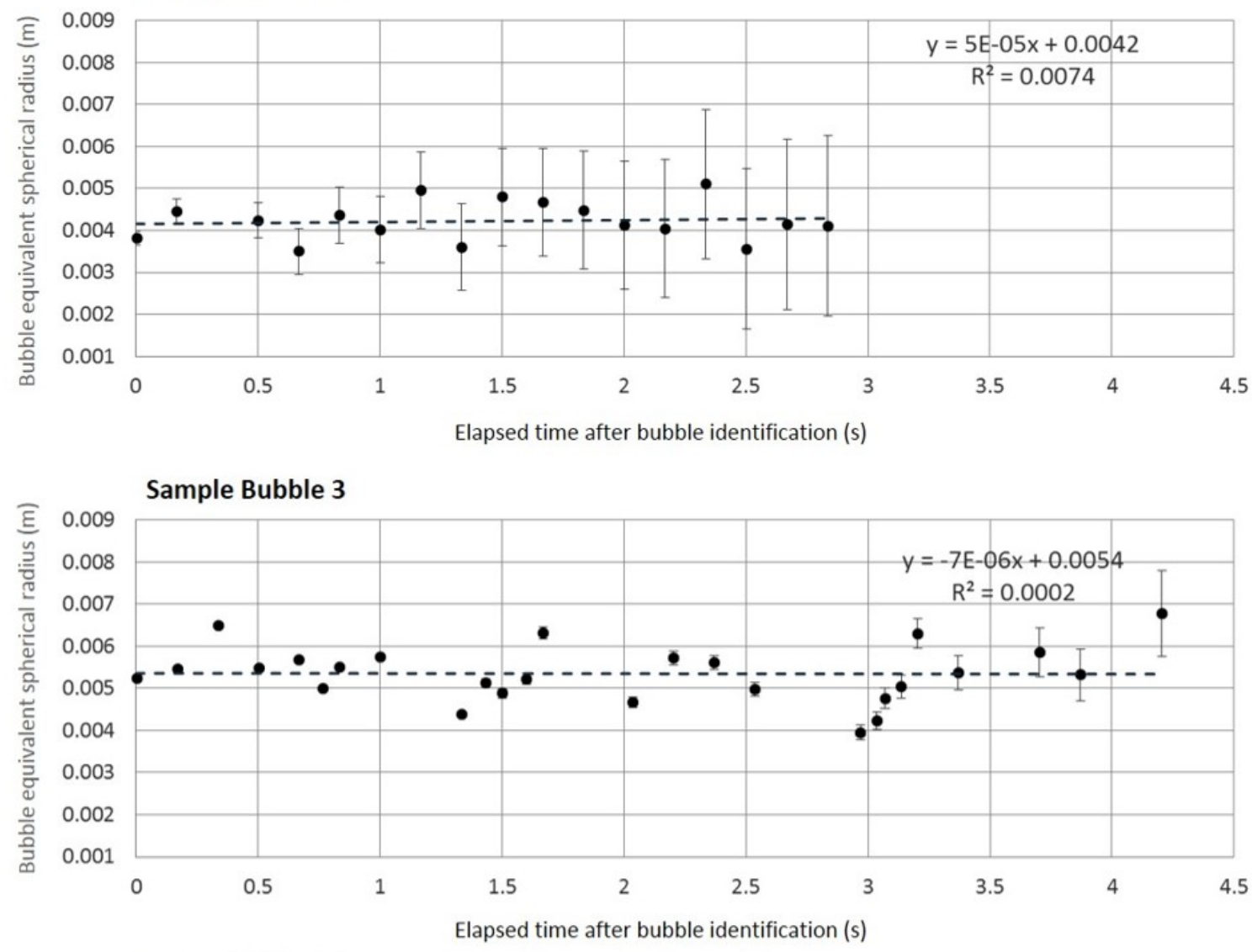

Figure 3.13: Three distinct bubbles have been tracked over multiple frames of the DTIS footage. The observed radius of the each bubble has been plotted with maximum and minimum uncertainties. Linear trends have been fitted through each plot with the corresponding equation and $\mathrm{R}^{2}$ in the top-right of each graph. 


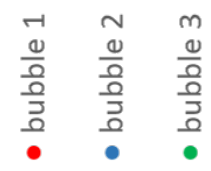

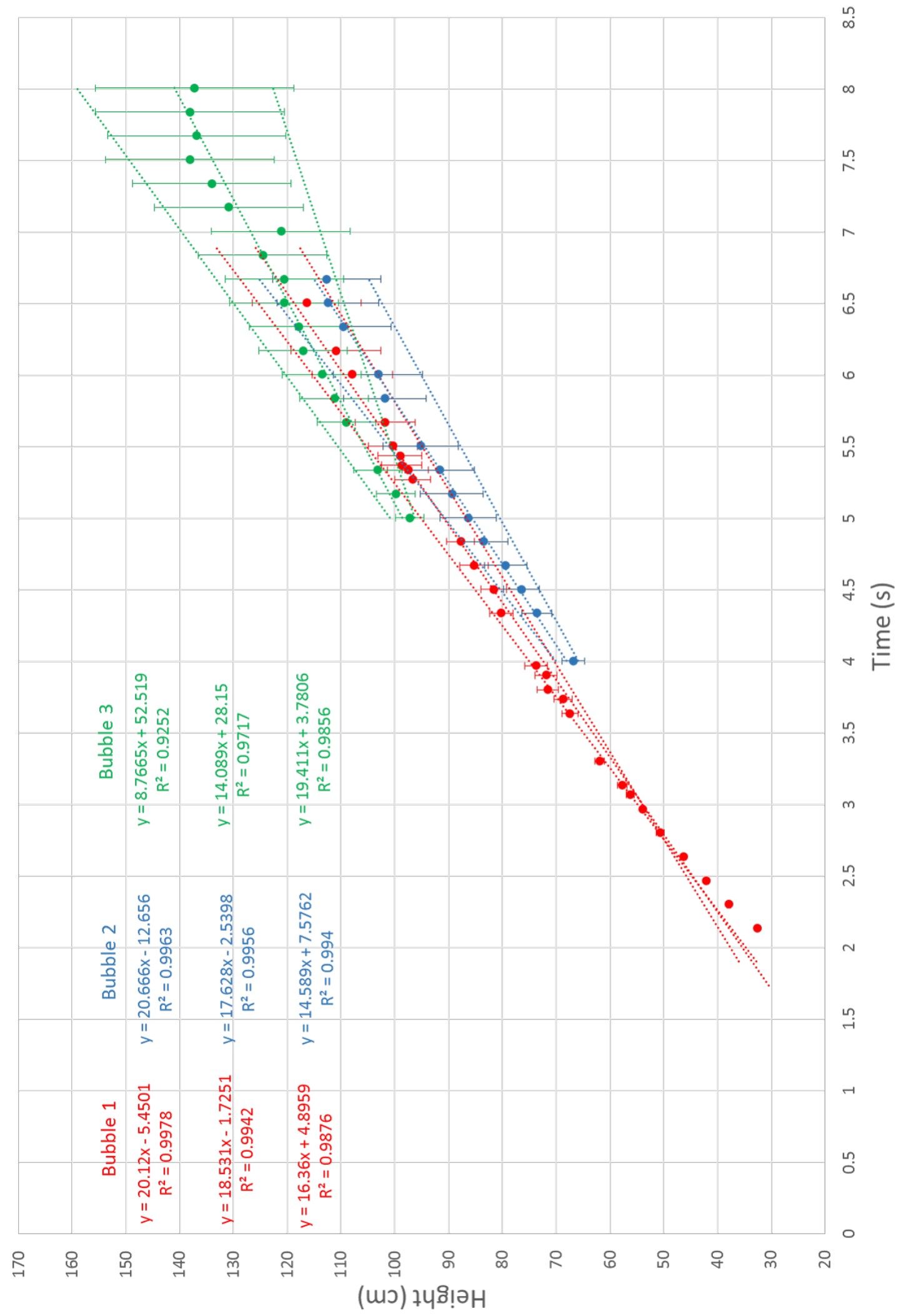

Figure 3.14: Elevation distances above the seafloor have been acquired for the bubbles 1 (red), 2 (blue) and 3 (green) with maximum and minimum uncertainty values. Linear trends have been fitted each series with corresponding equations. 
Experimental Bubble Rise Rates

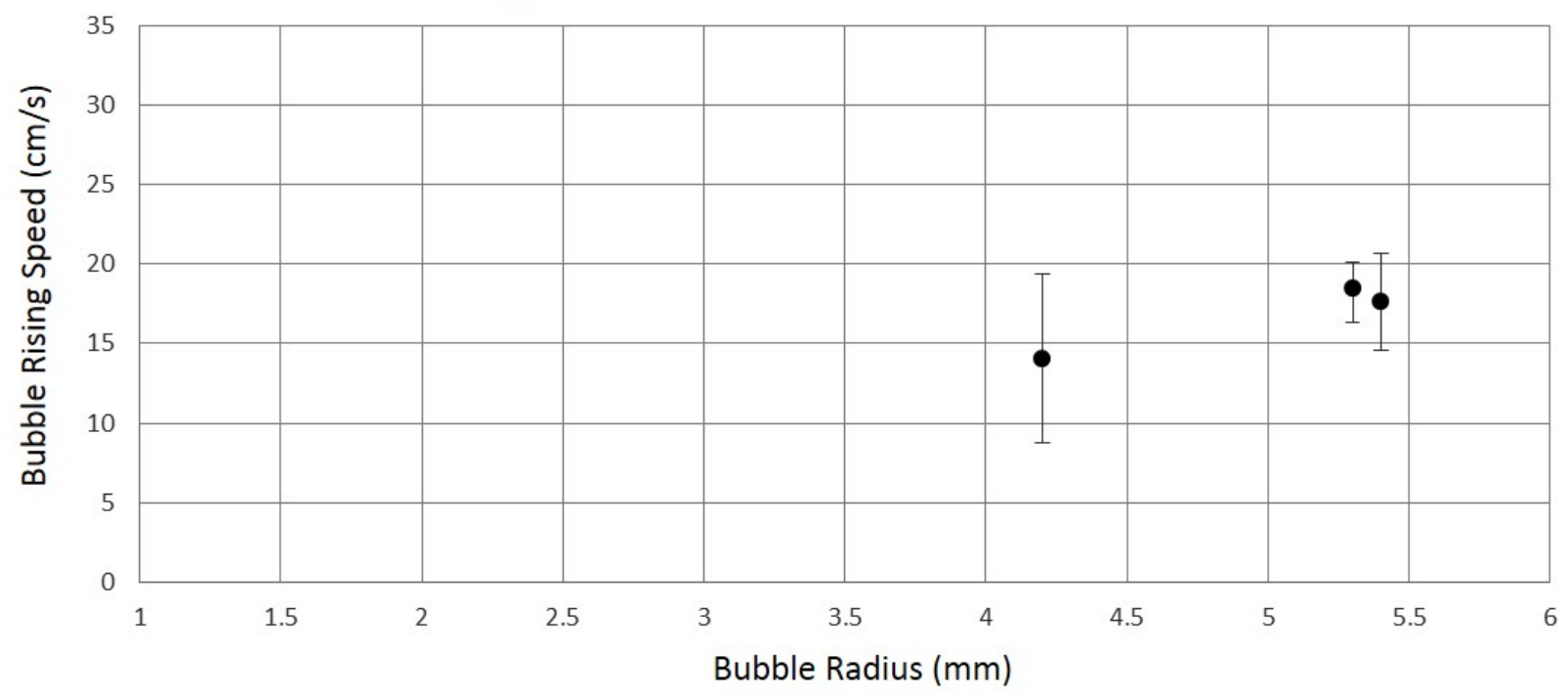

Figure 3.15: Plotted values of bubble size and respective rise rates from Figure 3.13 \& 3.14 


\subsection{Singlebeam flare profiles}

All singlebeam data were analysed for flares using the program 'EchoAnalysis', which was modified by Yoann Ladroit for this purpose. Of the identified flares, six have been selected (Figure 3.16) as examples that were not intruded by fish or plankton and that display a representative range of different flare sizes.

During the data collection stage, Flare F was identified as a much larger flare in comparison to the majority that were surveyed. As flares of this size are more likely to comprise methane that is transported to the atmosphere, the flare was resurveyed multiple times. Three of these repeat surveys produced good coverage of the flares, which are displayed in Figure 3.16 as Flare F1, F2 and F3. 

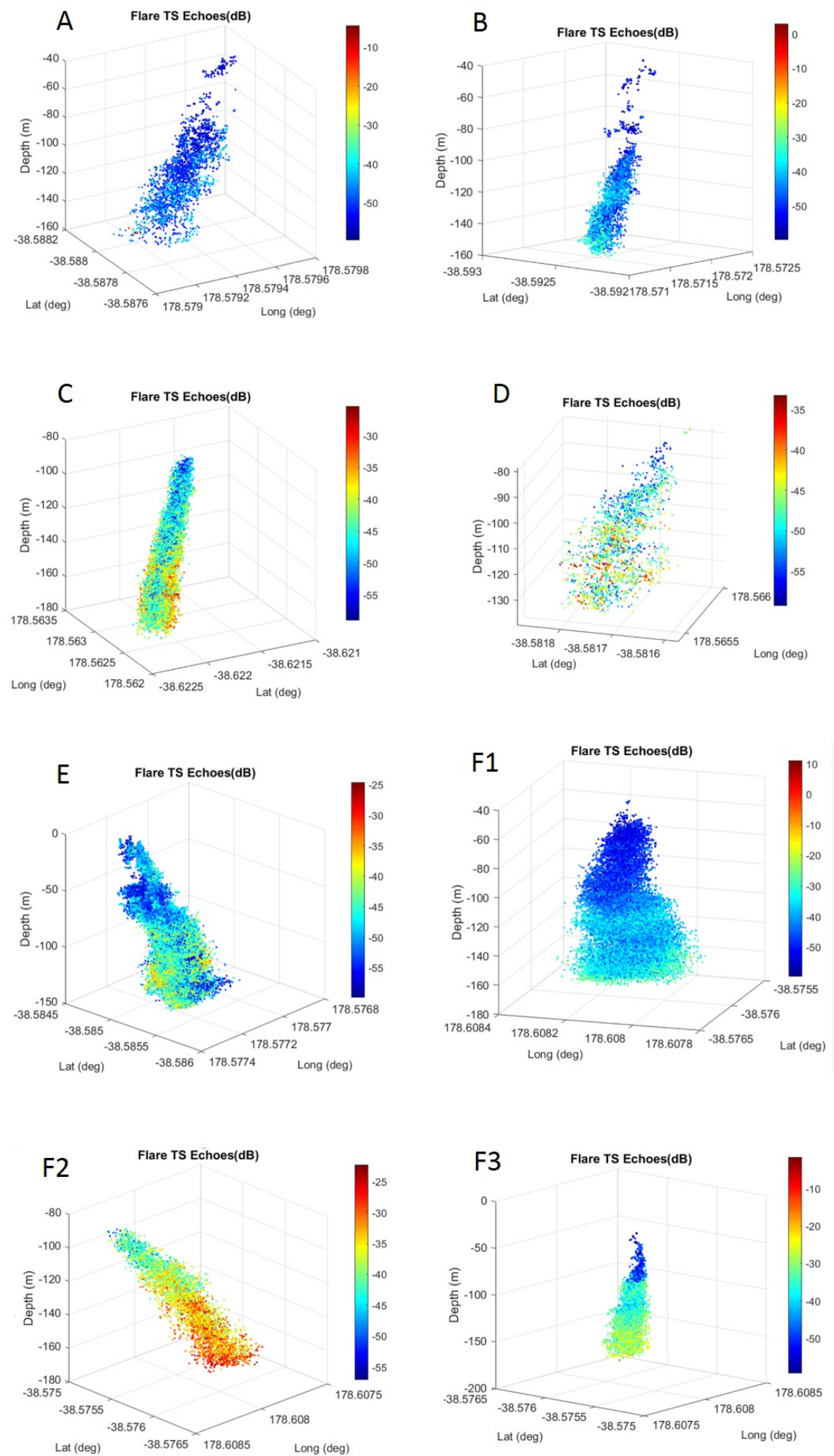

Figure 3.16: Acoustic flare profiles from the singlebeam data collected on TAN1505. Points are coloured by TS. Vertical scales start from the sea level at $0 \mathrm{~m}$ depth. 


\section{Discussion}

This thesis has focused on developing computational techniques for more accurately evaluating the parameters of rising bubbles in submarine-seep plumes. These techniques have been developed to better constrain the rate at which submarine seeps output gaseous methane. In the following discussion, the results of these techniques are compared to previously published results from related studies. In some cases, we have made inferences as to how estimations of seep output from current models could be improved with the use of our new techniques. The experimental results that have been produced for this thesis will be useful for better constraining regional approximations of the rates at which methane is escaping from entire seep fields and globally.

\subsection{Bubble sizes}

Experimental bubble-size distributions estimated in Section 6.3--6.11, are plotted against published experimental bubble-size distributions (Leifer and Patro, 2002; Muyakshin and Sauter, 2010; Greinert and Nützel, 2004; Römer et al., 2011; Sahling et al., 2009; Ostrovsky, 2003) in Figure 4.1. The size range of seep bubbles that we have measured lies between other video analysis bubble measurements made by Greinert and Nützel (2004) of artificially generated bubbles and Sahling et al. (2009) of seeps in the black sea. In comparison to the rest of the published bubble-size distributions, the size range of our bubbles more closely relates to larger bubble-size models.

Other published bubble-size distributions also cover a wider range of bubble sizes than we have observed (Figure 4.1). The absence of smaller bubbles in our distribution is likely due the resolution of the video footage, which limits what can accurately be defined as a bubble. Bubbles smaller than $\sim 6 \mathrm{~mm}$ were not identified in the video footage, but are likely still present in the bubble plume. Bubbles larger than $15 \mathrm{~mm}$ are also absent from our bubble size measurements. The absence of larger bubbles may be due to the limited period that we were able to observe the plume of seep bubbles. Larger bubbles likely occur less frequently (based on the low number of observations in Figure above $\sim 16 \mathrm{~mm}$ ) so it is possible that the $4 \mathrm{~s}$ recording window was not sufficient to observe bubbles of these sizes. Realistically, bubbles emitted from seeps along the Hikruangi Margin survey area likely range between $\sim 1 \mathrm{~mm}$ and $\sim 20 \mathrm{~mm}$ 


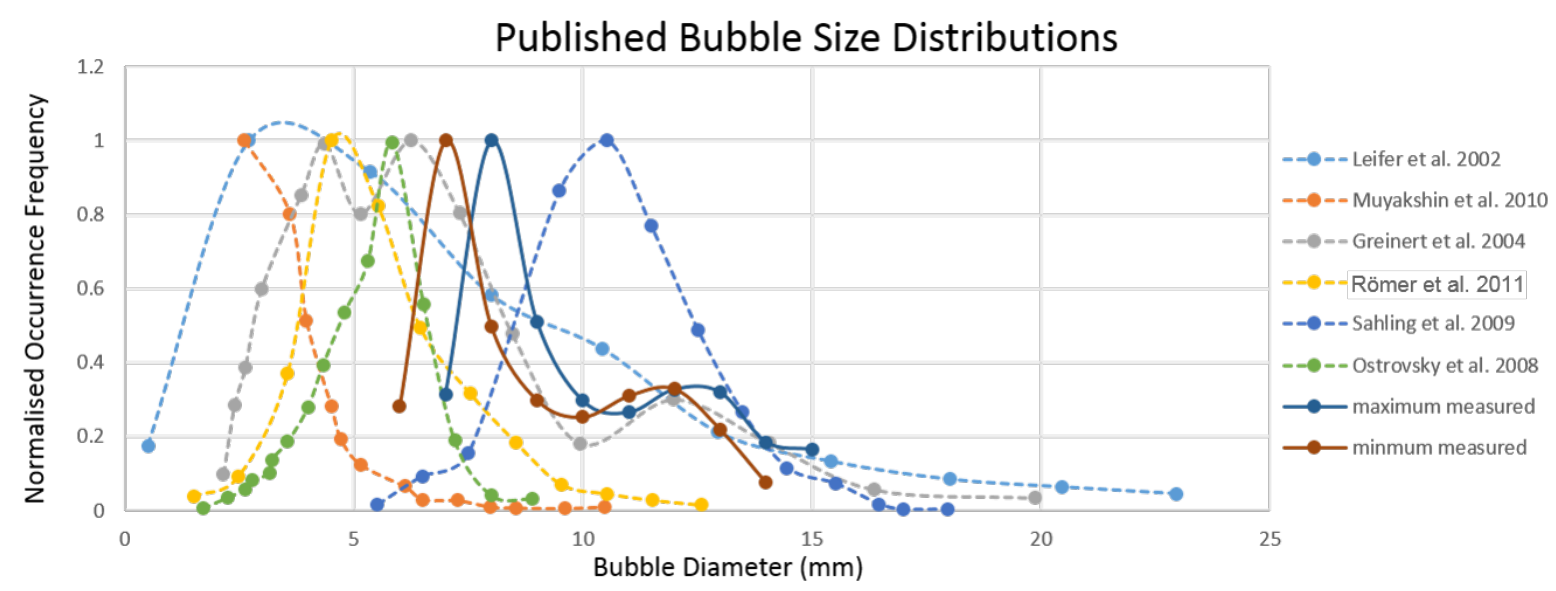

Figure 4.1: Maximum uncertainty (blue filled) and minimum uncertainty (red filled) bubble-size distributions have been plotted against previously published bubble-size distributions (Leifer and Patro, 2002; Muyakshin and Sauter, 2010; Greinert and Nützel, 2004; Römer et al., 2011; Sahling et al., 2009; Ostrovsky, 2003) (dashed).

as the other experimental observations predict.

It is noted that each different technique for calculating bubble-size distributions have used different methods for scaling the observed bubble sizes to the true bubble sizes. The apparent differences in bubble-size measurements across the published results may be a product of either the different scaling methods used or genuine differences in bubble sizes. Genuine differences in bubbles sizes may occur due to differences in physical conditions (e.g. temperature and pressure) at different seep locations.

\subsection{Characteristics of bubble ascent through the water column}

\subsubsection{Irregular rise paths}

Plotted bubble radii for three bubbles that have tracked over a succession of frames (see Figure 3.13) appear to sinusoidally vary in size over the course of their ascent. As it is unexpected for bubbles to noticeably change size over the first few metres of their ascent (McGinnis et al., 2006), it is more likely that these fluctuations are due to horizontal bubble movements rather than real size changes. As bubbles travel further from and closer to the camera during their rise, they appear respectively smaller and larger. These sinusoidal fluctuations are clear in Figure 4.2, where a spline has been fitted to the points in Figure 3.13. It can be seen in Figure 4.2 that fluctuations in bubble size occur for approximately the same radius differences over similar time scales. Consistent deviations around a central rise path support theoretical models claiming 
elliptical bubbles rise in either helical (Miyagi-Kôgakuhakusi, 1927) or zig-zag (Datta et al., 1950) patterns. Unfortunately the resolution of the recorded size fluctuations is not high enough in this case to confirm which of either the Miyagi-Kôgakuhakusi (1927) or Datta et al. (1950) bubble-rise theories is applicable in this case.

\subsubsection{Adjustments to theoretical bubble-rise rates}

Bubble-rise rate measurements obtained from a time series of tracked bubbles have been plotted against other theoretical methane-bubble rise rates (see Figure 4.3). Theoretical methane-bubble rise rates have been devised for both clean and dirty bubbles (where dirty bubbles are covered in a surfactant coating and clean bubbles are completely uncontaminated). The uncertainty limits of the bubble-rise rates we have tracked overlap marginally with the theoretical rise-rate model devised by Leifer et al. (2000). For all bubble-rise rate models (Mendelson, 1967; Woolf and Thorpe, 1991; Woolf, 1993; Leifer et al., 2000; Leifer and Patro, 2002), experimental measurements that we have made are consistently slower than the theoretical predictions.

It has already been established in Section 3.3.1 that Sample Bubbles 1, 2 and 3 (Figure 3.14) are travelling at their terminal rise velocities. As the theortical models plotted in Figure 4.1 are all based on terminal-rise velocities, we are able to make direct comparisons between experimental and theoretical rise rates. It has also already been shown in Figure 4.1 that experimental radius measurements are consistent with other seep bubble measurements, implying there is minimal horizontal error for points plotted in Figure 4.3. As bubble size and acceleration are both in agreement with other models, we have assumed that disparities between experimental and theoretical bubble-rise rates are exclusively due to differences in bubble-rise velocity.

Leifer et al. (2000) have predicted that bubble-rise rates are overestimated in the current theoretical bubble-rise rate models. This overestimation is thought to arise from assumptions in dirty-bubble theoretical rise-rate models that have neglected the slowing effects of bubble oscillations (see Figure 4.4). Oscillations slow a bubble's ascent as energy from their rise is transferred into perpendicular oscillatory motions. The presence of surfactants around bubble walls act to suppress these oscillations. Due the damping effect of surfactants, most bubble rise velocity models neglect oscillations of dirty bubbles completely, assuming they behave as solid spheres. Since dirty bubble oscillations are suppressed, but not completely eliminated, previously predicted dirtybubble rise-rate models may only be accurate for bubbles where $\mathrm{r}<600 \mu \mathrm{m}$ (where oscillations are negligible) (Leifer et al., 2000). Because the slowing effects of oscillatory motions in dirty bubbles have not been taken into account for the models plotted in Figure 4.3 it is possible that the differences in observed and predicted values are a 

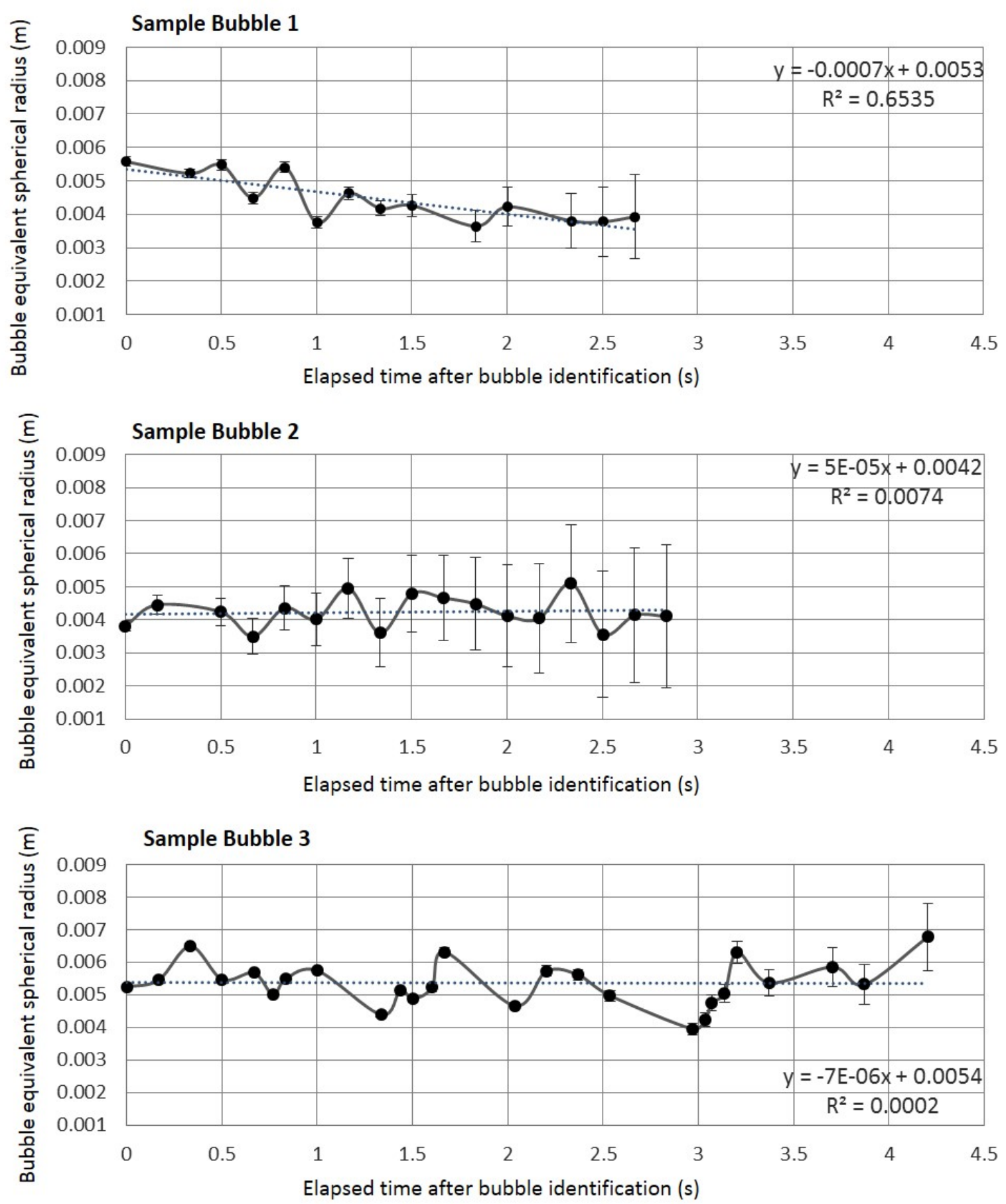

Figure 4.2: Three distinct bubbles have been tracked over multiple frames of the DTIS footage. The observed radius of the each bubble has been plotted with maximum and minimum uncertainties. Linear trends have been fitted through each plot with the corresponding equation and $\mathrm{R}^{2}$. A spline has also been fitted to the measured bubble-size measurements to show sinusoidal variations around the linear trend. 


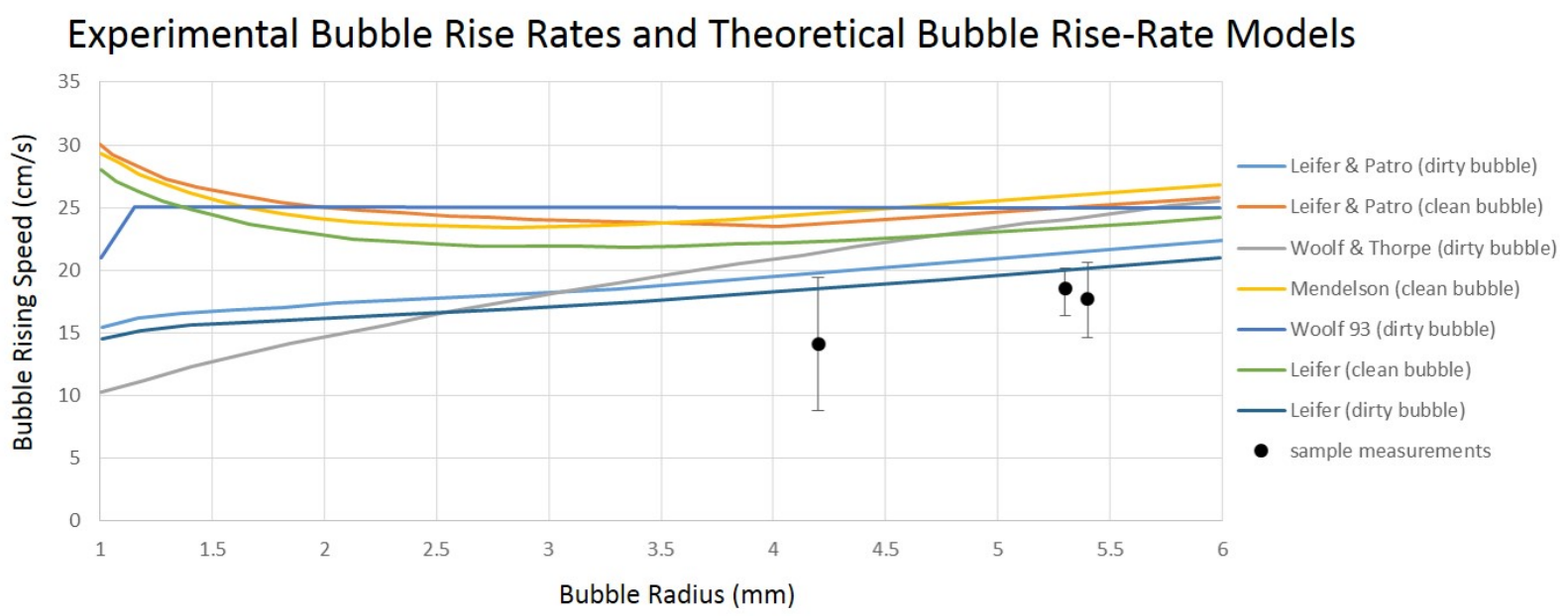

Figure 4.3: Previously published theoretical models for bubble-radius dependant rise speeds (Leifer et al., 2000; Leifer and Patro, 2002; Woolf and Thorpe, 1991; Woolf, 1993). Rise speeds from sample bubbles (Figure 4.5) have been plotted against these models with vertical uncertainties. Bubble-size uncertainties are negligible on the scale of this graph.

product of oscillatory slowing effects.

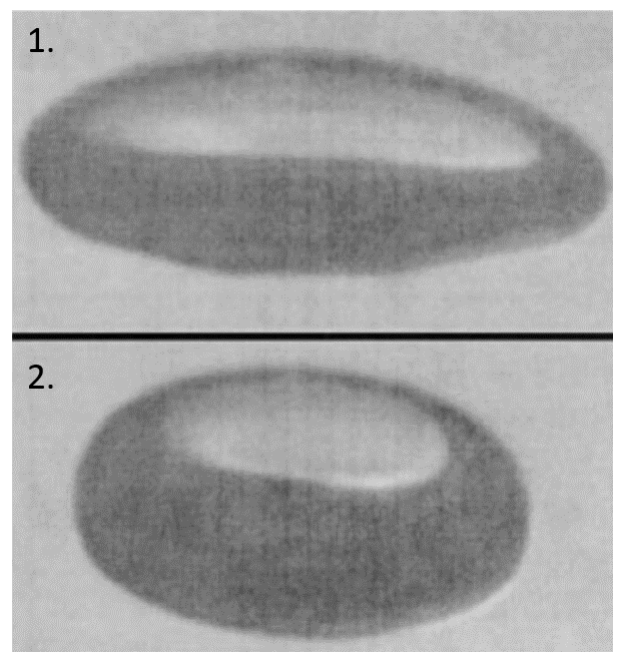

Figure 4.4: A bubble imaged at two different stages of oscillation. In the usual case, the bubble profile (as in 1) will expand along its two major axes and shorten along its minor axis (as in 2), then return to its original state (as in 1). This is characteristic of elliptical bubbles and occurs continuously over a seep bubble's ascent.

In all bubble-rise rate theoretical models, dirty bubbles rise more slowly than clean bubbles (Leifer et al., 2000; Leifer and Patro, 2002; Woolf and Thorpe, 1991; Woolf, 1993). Measured rise rates that we have computed for the sample bubbles are consistently lower than theoretical model predictions. The only apparent reason for these discrepancies are due to solid-sphere assumptions made in dirty bubble rise-rate models. Due to the combined evidence of slower-rising bubbles and recognised overestimations that 
are exclusive to dirty bubble rise-rate models, we have characterised the experimental bubbles as dirty bubbles.

In order to construct a bubble-rise rate model that more accurately represents the measured rise rates of the sample bubbles, we have approximated the amount of overestimation in the Leifer et al. (2000) bubble rise-rate model. By vertically shifting the Leifer et al. (2000) model so that the distribution fits the experimental measurements and their lower uncertainties, we have acquired bubble-rise rate models consistent with our results (Figure 4.5). If the vertical offset represents the slowing effect of impurities then impurities cause a slowing of $3-3.7 \mathrm{~cm} / \mathrm{s}$.

\section{Experimental Bubble Rise-Rate Models}

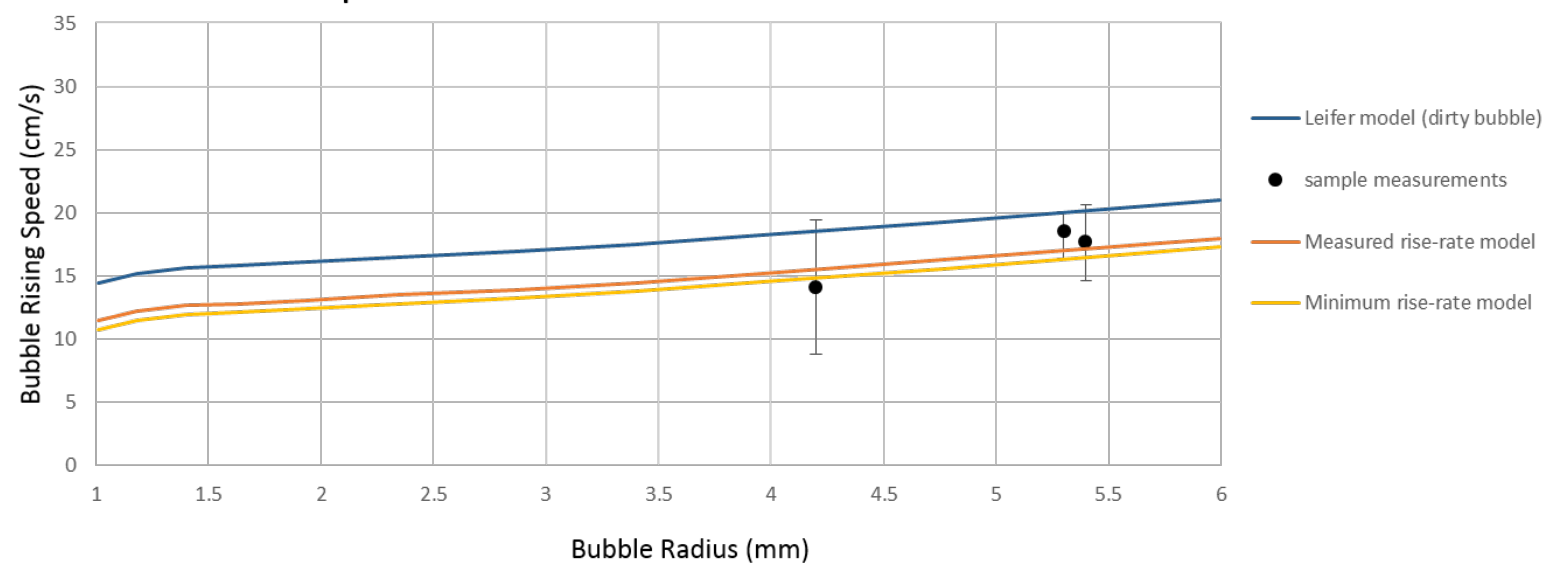

Figure 4.5: The bubble rise-rate model from (Leifer et al., 2000) has been vertically shifted to account for overestimations. The adjusted models better represent our observed experimental bubble-rise rates. 


\subsection{Predictions of methane flux}

We combined bubble rise-rate models (Figure 4.5) with experimental bubble-size distributions (Figure 3.12) and singlebeam flare profiles (Figure 3.16) to estimate the flux of methane emitted from the surveyed submarine seeps. Methane fluxes are calculated using the FlareFlow Matlab module (Veloso et al., 2015). For each flare loaded into the FlareFlow module, methane flux values are acquired for both experimental maximum and minimum dirty bubble rise-rate models applied to both the maximum and minimum bubble-size distributions. Methane flux rates are also evaluated for two depth windows of each flare: (1) from $5 \mathrm{~m}$ above the seafloor to the highest vertical point of the flare (2) between $5 \mathrm{~m}$ and $10 \mathrm{~m}$ above the seafloor. The lowest $5 \mathrm{~m}$ of acoustic data are eliminated in both cases as it likely contains erroneous data (Veloso et al., 2015). Elevated carbonate mounds and other seafloor echoes may be present in these first $5 \mathrm{~m}$, so neglecting this section will eliminate any target strength (TS) values not produced by bubbles.

To estimate the number and sizes of bubbles in a seep plume within a chosen volume, bubble-echoed TS values are correlated to bubble radius. These correlations are based on the percentage of energy that a bubble scatters (proportional to the ratio of $\mathrm{TS}_{\text {received }} / \mathrm{TS}_{\text {emitted }}$ ) when insonified by a singlebeam echosounder ping (detailed in Section 2.7). To obtain mean bubble sizes for all bubbles within a segment of a flare, TS values are averaged for echoes within a chosen volume (where $\overline{\mathrm{TS}}$ is the resultant averaged value). As bubbles of different radii are insonified by specific frequencies of sonar energy, only a particular size range of bubbles is sampled by the chosen $38 \mathrm{kHz}$ frequency beam. The remaining proportion of bubbles with radii outside of the insonfied range are estimated computationally using the maximum and minimum bubble-size distribution curves (Figure 3.12). The selected depth window for calculating $\overline{\mathrm{TS}}$ has a direct influence on the calculated size and number of the imaged seep bubbles. Calculations of $\overline{\mathrm{TS}}$ for flare segments between $5 \mathrm{~m}$ and $10 \mathrm{~m}$ above the seafloor are considered representative of the radii of bubbles that are being released straight from the source of the seep (Veloso et al., 2015). Calculations for flare segments extending from $5 \mathrm{~m}$ above the seafloor to the highest point of the flare will result in a smaller apparent bubble-size distribution, as $\overline{\mathrm{TS}}$ is calculated for the included weaker echoes at higher flare elevations.

Undissolved methane fluxes for different depths windows of the flares displayed in Figure 3.16 are tabulated in Table 4.1 \& ??. It is noted in these results that the majority of analysed seeps discharge gaseous methane at rates of $\sim 37-286 \mathrm{~mL} / \mathrm{min}$. However, there is also evidence that higher-energy seeps are capable of producing 
gaseous methane at rates of $\sim 1115-3371 \mathrm{~mL} / \mathrm{min}$.

\begin{tabular}{|c|c|c|c|c|}
\hline \multicolumn{5}{|c|}{ Methane Flux Measurements Based On Minimum Bubble Rise Rate } \\
\hline \multirow[t]{2}{*}{ Flare } & \multicolumn{2}{|c|}{ Maximum flux (mL/min) } & \multicolumn{2}{|c|}{ Minimum flux (mL/min) } \\
\hline & $\begin{array}{l}5 \mathrm{~m} \text { above the } \\
\text { seafloor - top } \\
\text { of the flare }\end{array}$ & $\begin{array}{l}5-10 \mathrm{~m} \\
\text { above the } \\
\text { seafloor }\end{array}$ & $\begin{array}{l}5 \mathrm{~m} \text { above the } \\
\text { seafloor - top } \\
\text { of the flare }\end{array}$ & $\begin{array}{l}5-10 \mathrm{~m} \\
\text { above the } \\
\text { seafloor }\end{array}$ \\
\hline A & 32.4 & 44.2 & 26.1 & 37.0 \\
\hline $\mathrm{B}$ & 110.0 & 286.1 & 92.1 & 239.4 \\
\hline $\mathrm{C}$ & 172.4 & 281.3 & 135.6 & 226.3 \\
\hline $\mathrm{D}$ & 46.9 & 53.8 & 37.7 & 42.3 \\
\hline $\mathrm{E}$ & 48.9 & 60.0 & 39.4 & 48.3 \\
\hline F1 & 247.5 & 1386.4 & 199.2 & 1115.2 \\
\hline $\mathrm{F} 2$ & 775.2 & 2105.8 & 623.9 & 1693.9 \\
\hline F3 & 751.1 & 3370.9 & 604.5 & 2711.5 \\
\hline
\end{tabular}

Table 4.1: Minimum values for flux calculations for each of the flares displayed in Figure 3.16. Flare F1, F2 and F3 are three acoustic profiles of the same flare.

\subsection{Multiple bubble exits at the seafloor}

Results that have been obtained from vertically summing echo intensity values for acoustic data collected over a prominent flare (see Figure 3.4) are displayed in Figure 3.6. Staggering these summations across $10 \mathrm{~m}$ elevation windows between the seafloor (at $0 \mathrm{~m}$ ) and sealevel (at $164 \mathrm{~m}$ above the seafloor) gives a detailed view of the changing flare intensity at shallowing depths. Based on these summed intensity slices, we have made the following seep-bubble preservation interpretations:

It can be seen in first two surfaces (0 to $10 \mathrm{~m}$ and 10 to $20 \mathrm{~m}$ in Figure 3.6) that there are multiple high-amplitude zones, each indicative of bubble escape from the seafloor. Multiple zones suggest that the seafloor opening at the base of the seep is not a distinct 
aperture, more likely a patchy system of localised seafloor cavities. It can be seen on a cross-sectional view of the flare (see Figure 3.4) that there is an elevated knoll feature at the base of the flare. Based on video underwater video footage over the survey region and previous studies of knoll features along the Hikurangi Margin (Liebetrau et al., 2010), it is expected that this knoll feature is a carbonate mound.

Carbonate mounds form at the base of active seeps through microbially mediated anaerobic oxidation of emitted methane (León et al., 2007). As they begin to cover the area of seep-fluid release, they may influence the output of fluid. This is likely what is observed in Figure 4.6, where intensity indicators for Flares 1-5 (from Figure 3.6) appear to escape from multiple exit points around a central carbonate mound. The predominant central flare (Flare 1) above the central mound is surrounded by smaller flares that have also formed smaller mounds. The comparatively smaller proximal mounds suggest that the associated seeps have been either less active or are younger than the central seep. Both of these observations make it likely that proximal seeps have been formed as a result of fluid redistribution by carbonate capping of the central seep. There is also evidence of smaller-scale gas seepage (labelled in Figure 4.6) escaping from around the central carbonate mound.

Similar evidence of fluid redistribution have been documented along the Gulf of Cadiz (León et al., 2007). Figure 4.7 illustrates the structural fluid-redistribution patterns formed by carbonate precipitations. Seep fluid is able to travel through separated passageways of higher relative permeability, which results in multiple sites of localised fluid expulsion. Seafloor video footage and studies by Liebetrau et al. (2010) of the region has revealed multiple other carbonate mounds around active seeps. These observations suggest that similar fluid redirection is occurring at multiple active seeps across the Hikurangi Margin. The presence of these carbonate mounds is also indicative of long existing seepage. Studies of mound formation across Svalbard (Berndt et al., 2014) and other parts of the Hikurangi Margin (Liebetrau et al., 2010) have deduced that it takes thousands of years for seeps to accumulate carbonate mounds. As there is similar evidence of seep-accumilated carbonate mounds in our study area, we have inferred that seep activity has been occurring over the last few thousand years.

\subsection{Bubble survival during ascent}

\subsubsection{Relative survival rates of an assemblage of seeps}




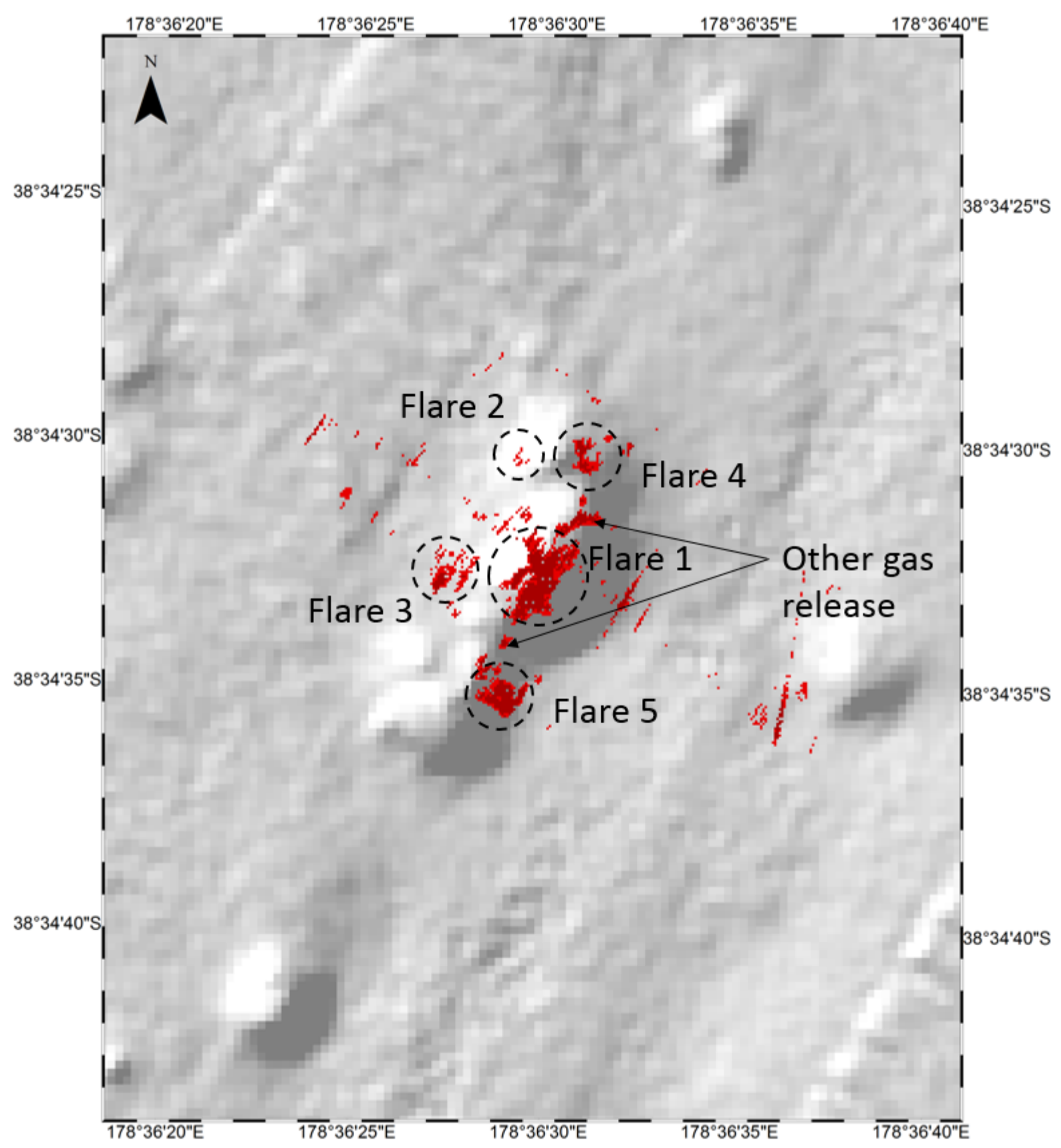

Figure 4.6: Flare indicators from the 0-10 m summed-intensity surface in Figure 3.6 have been superimposed over the bathymetry. The zones of escaping seep-fluid correlate with aggregated knoll features (identified as carbonate mounds). Smaller zones of minor gas release that were not identified as defined seeps in Figure 3.6 are also present around the carbonate mounds.

We have analysed the five flares labelled 1-5 in Figure 3.6 to assess the intensity depletion of flares in close proximity of each other at shallowing depths. The overall decrease in flare diameter with increasing elevation for all labelled flares indicates there is a decrease in flare intensity at shallowing depths.

Two of the peripheral flares (Flares $2 \& 3$ ) terminate within the first $40 \mathrm{~m}$ above the 


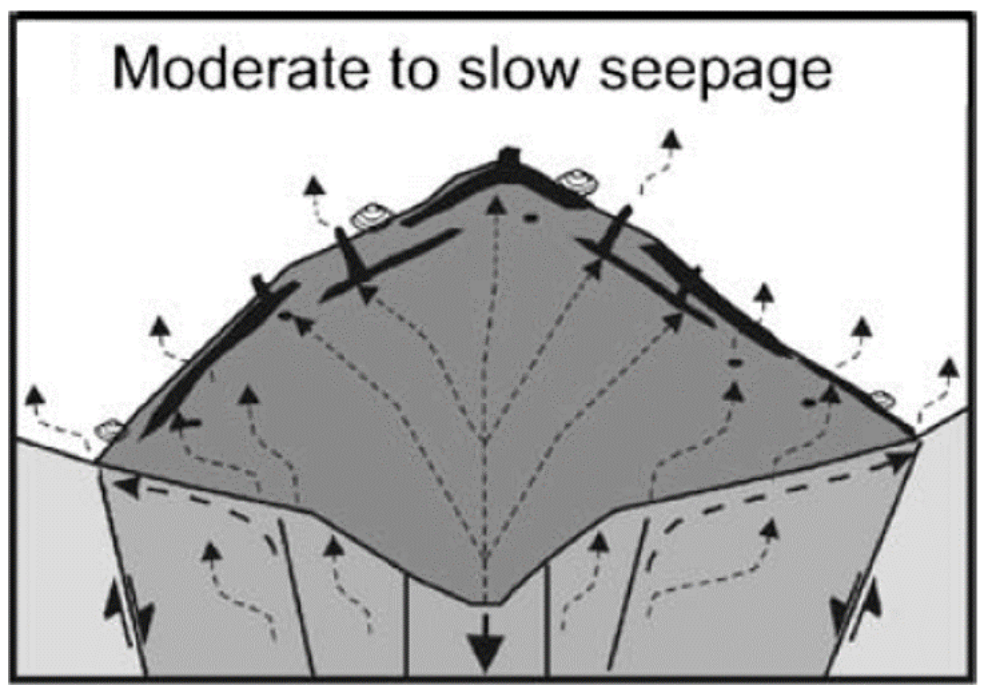

Figure 4.7: Illustration of a carbonate mound on the seafloor from (León et al., 2007). Dotted arrows show the flow paths of rising seep fluid, which is redirected through more permeable parts of the mound.

seafloor $(120 \mathrm{~m} \mathrm{bsl})$. The other two remaining proximal flares (Flares $4 \& 5$ ) are terminated somewhere between $60 \mathrm{~m}$ and $80 \mathrm{~m}$ above the seafloor $(84 \mathrm{~m} \mathrm{bsl})$. There is remaining acoustic evidence of the central flare (Flare 1) for the entire height of the watercolumn. The survival of seep bubbles that have been acoustically imaged in Flare 1 is likely due the preserving effects of the surrounding bubbles of the proximal seeps and those within Flare 1. Section 1.4 details how concentrated bubbles dissolve at a lower rate than sparse bubbles, which increases their likelihood of surfacing.

\subsubsection{Bubble survival in a single seep plume}

We have used the plotted histograms (see Figure 3.7) for the staggered summed intensity surfaces (see Figure 3.6) to make a first approximation of the proportion of undissolved seep fluids that survives the ascent of the water column. Plotted histograms of summed intensity in Figure $3.7 \& 3.9$ show two distinct peaks in the occurrences of two separately binned intensity values. These peaks show that summed-intensity values of $\sim-200 \mathrm{~dB} \&-1500 \mathrm{~dB}$ occur most frequently and there is comparatively little evidence of other summed-intensity values. Both of these peaks gradually decrease in occurrence at shallower depths.

The regions of the flare where these peak summed-intensity values occur can be found from the mapped summed intensity surfaces in Figure 3.6. Analysing the changing summed intensity of flares across surfaces in Figure 3.6 reveals that peak-summed intensity values of $\sim-1500 \mathrm{~dB}$ are situated in the core of flares and peak-summed intensity values of $\sim-200 \mathrm{~dB}$ are situated at the rim and uppermost parts of flares. As both peak occurrence values of $\sim-200 \mathrm{~dB} \&-1500 \mathrm{~dB}$ are consistent with different 
parts of the acoustically imaged seep, we have inferred that these indeed correlate with echoed responses of rising bubbles.

Since bubbles of comparable sizes are expected to echo similar proportions of acoustic energy, it would be intuitive for there to be only one peak summed-intensity value in Figure 3.7 \& Figure 3.9 that represents bubbles from all parts of the seep plume. Instead of a bimodal bubble-size distribution, it is more likely that the second $\sim-1500 \mathrm{~dB}$ peak is from an increased concentration of bubbles of similar size to those echoing $\sim-200 \mathrm{~dB}$. Bubbles in the centre of a seep are more highly concentrated than those around the outside.

The reason that a more highly concentrated volume of bubbles would produce a higher echoed amount of energy is most likely due to multiple backscattering (Clay and Medwin, 1977). Sound energy echoing from bubbles may be additionally echoed off multiple other bubbles if bubbles are more closely spaced than the wavelength of the sonar pulse. As a first approximation (from the $30 \mathrm{kHz}$ sound pulse produced by the EM302 echosounder and assuming the speed of sound in seawater is $\sim 1500 \mathrm{~m} / \mathrm{s}$ ), bubbles less than $5 \mathrm{~cm}$ apart will produce a multiple backscattering effect. The higher number of echoes resulting from multiple backscattering will result in less scattering of energy and more being echoed back to the receiver. This likely produces the heightened acoustic response (of $\simeq-1500 \mathrm{~dB}$ ) at the core of flares where bubbles are most concentrated. The constitutive bubble of these two high and low summed-intensity regions have been termed concentrated and sparse respectively.

Peaks corresponding to concentrated and sparse bubbles in Figure 3.7 have been plotted in Figure 4.8. It is apparent from both the concentrated and sparsely bubble-populated parts of the flares that summed intensity seems to decrease at an steadily-growing rate in the first $70 \mathrm{~m}$ (from $164 \mathrm{~m}$ to $94 \mathrm{~m} \mathrm{bsl}$ ). Above $94 \mathrm{~m} \mathrm{bsl}$, flare depletion becomes more unpredictable and concentrated bubbles are lost completely. A steady decrease in flare intensity continues up until $\sim 10-20 \mathrm{~m} \mathrm{bsl}$ ). Above $\sim 10-20 \mathrm{~m}$ bsl there is a slight increase in summed intensity.

Figure 4.9 displays bubble dissolution models devised by McGinnis et al. (2006) for individual methane bubbles rising through water. Models for changing bubble size indicate that bubbles initially shrink due to the dissolution of methane, but then grow at shallower depths due to decreasing pressure (McGinnis et al., 2006). The subsequent contraction and growth of bubbles in the top $30 \mathrm{~m}$ of the water column is a possible explanation for the observed rise and fall of summed-intensity occurrences in the upper $30 \mathrm{~m}$ of Figure 4.9. Seep bubbles may shrink and expand due to a shift from a dissolution dominant to pressure dominant system. The reduction in bubble size will 
likely result in bubbles being undetectable in acoustic data over a short depth window. Subsequent bubble expansion in the upper-most part of the water column will allow the bubbles to again be acoustically detected.

Models of methane preservation within rising methane bubbles (McGinnis et al., 2006) are also plotted in Figure 4.9. Methane composition trends show that a large proportion of methane is depleted rapidly after bubbles come into contact with water. We have applied the methane dissolution curves to a 4.25 mm-radius bubble rising from $164 \mathrm{~m}$ water depth (depth of the flare in Figure 3.4 and analysed in Figure 3.6 and Figure 4.8) and plotted the results in Figure 4.10. The applied model for methane dissolution predicts that $15 \%$ of methane is preserved in a seep bubble of $4.25 \mathrm{~mm}$ radius rising through $164 \mathrm{~m}$ of water. It important to note that this is a minimum estimate in the case of rising seep bubbles, as the dissolution model is valid for a single rising bubble. Bubbles rising in clusters (as is the case for submarine seeps), will have a lower rate of methane reduction for each bubble (for reasons detailed in Section 1.4).

We have combined bubble methane dissolution models (Figure 4.10 derived from McGinnis et al. (2006) with approximated bubble depletion rates (Figure 4.8) to estimate the proportion of undissolved methane released from the seafloor that reaches the atmosphere. Methane dissolution models have revealed that $\sim 15 \%$ of any initially pure methane bubble is expected to still be present at the surface. Of the initial discharge of bubbles, we have used the acoustic observations in Figure 4.8 to approximate the reduction in bubble quantity. The occurrence of echoes indicative of seep bubbles (for reasons outlined previously in this section) has decreased from 1600 to 25 in Figure 4.8, which suggests that $1.25 \%$ of the initial bubble quantity survives the ascent through the water column. Collating the proportions from the methane dissolution and bubble quantity depletion model predicts that $0.2 \%$ of the methane released by the flare imaged in Figure 3.4 reaches the atmosphere. 


\section{Depletion of undissolved seep fluid}

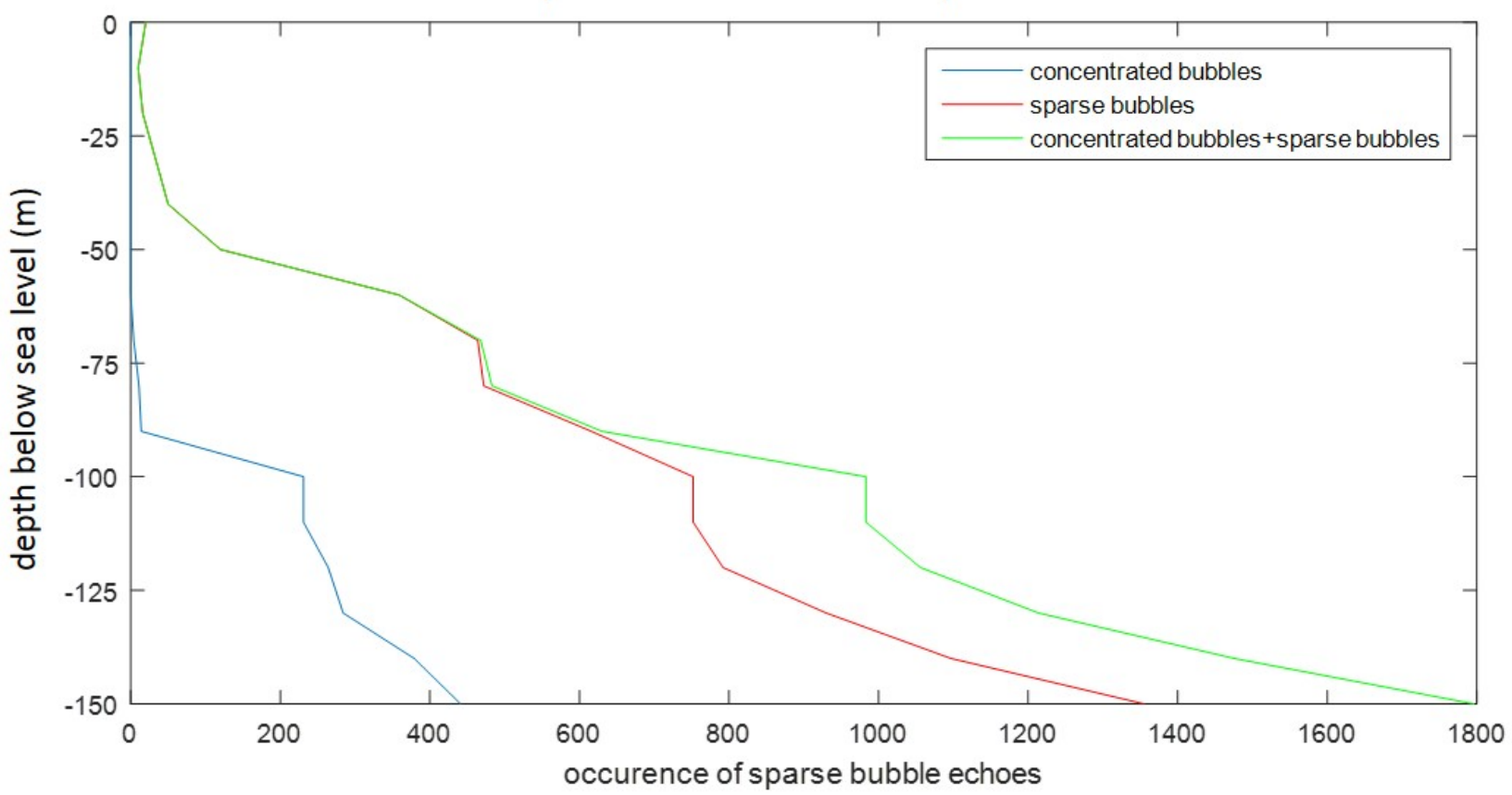

Figure 4.8: Occurrences of bubble-echo peaks from Figure 3.7 have been plotted against depth for sparse bubbles, concentrated bubbles and summed sparse and concentrated bubbles.
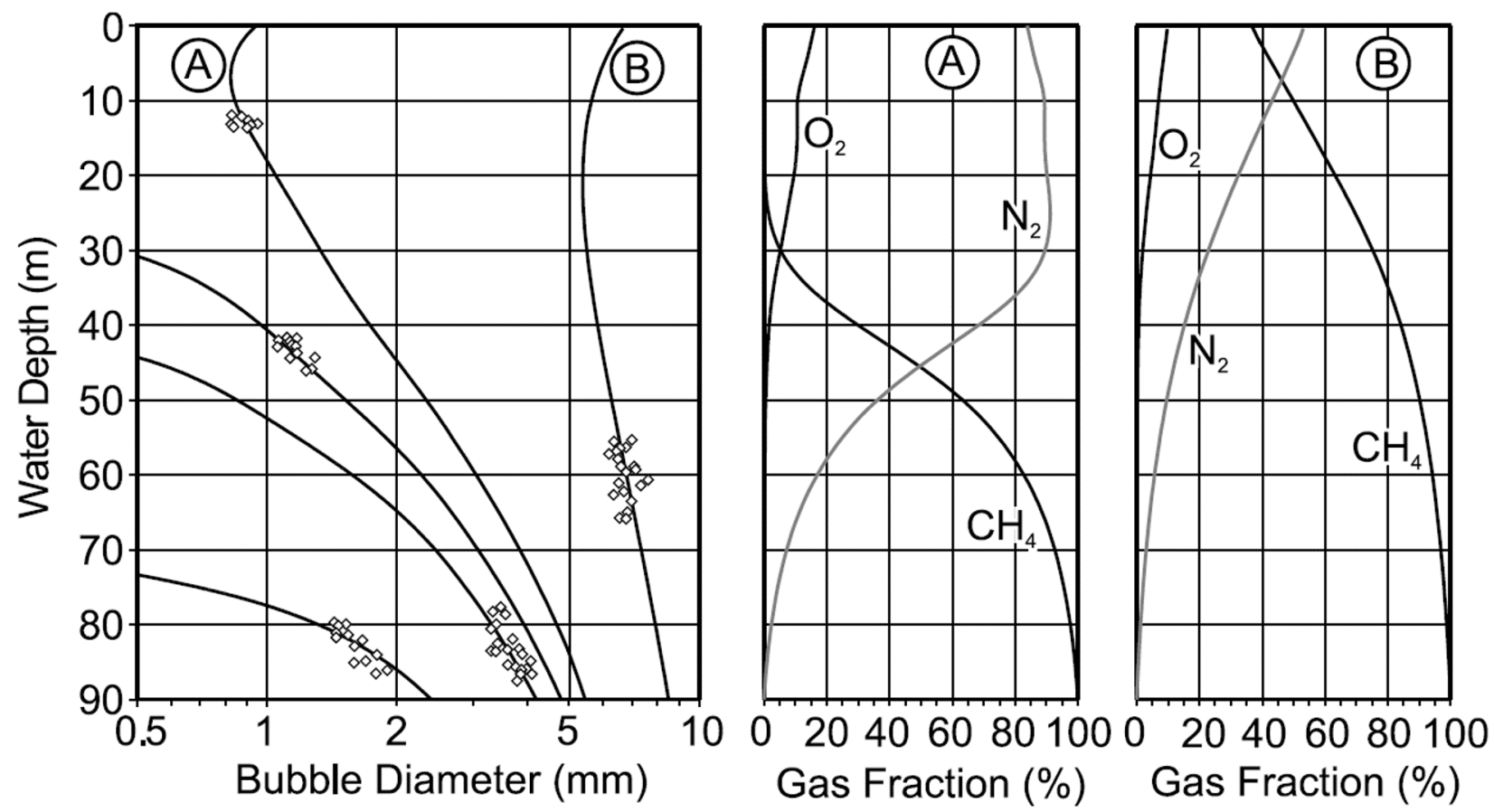

Figure 4.9: The bubble dissolution and pressure response model created by McGinnis et al. (2006) has been applied to bubble A (2.75 mm radius) and B (4.25 mm radius). The results show the changing bubble diameter and gas composition with shallowing water depth. 


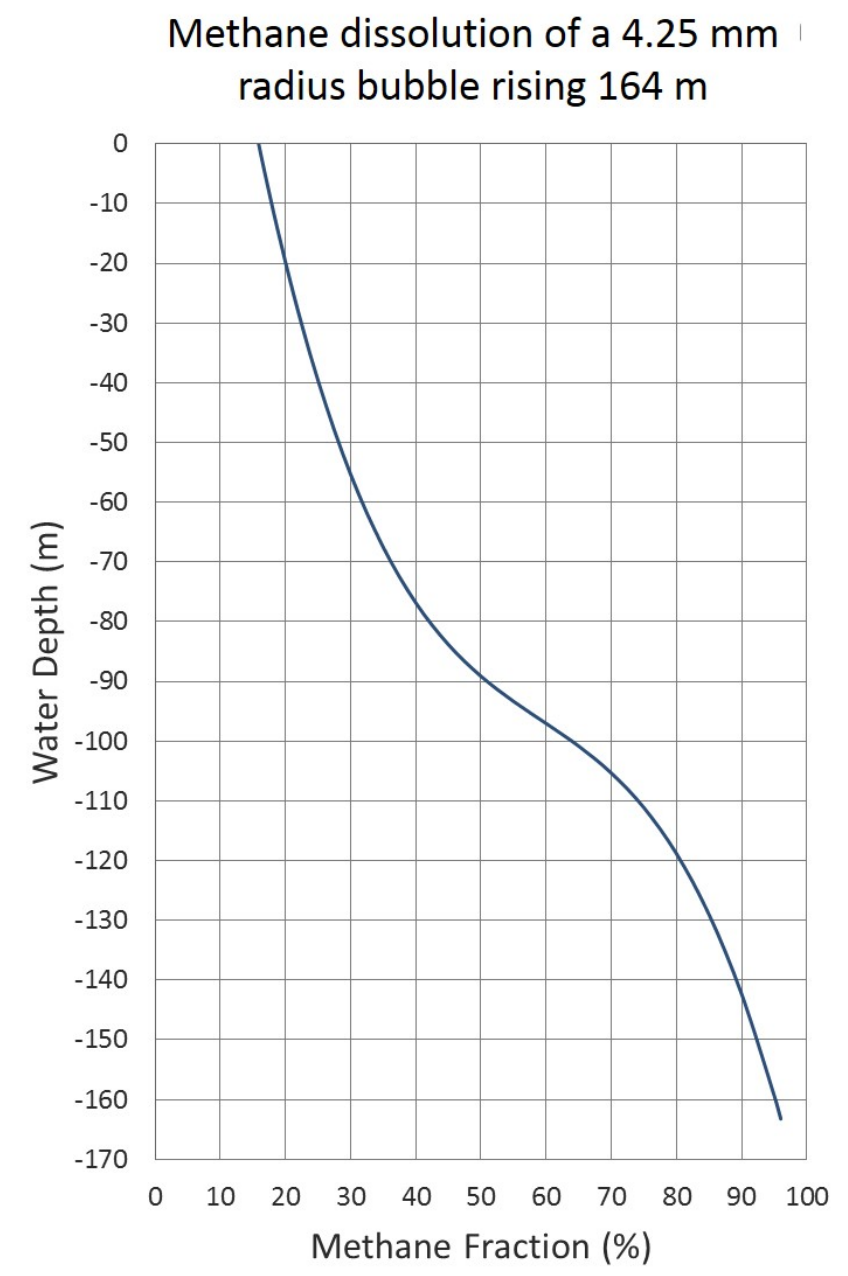

Figure 4.10: Methane depletion rate of a $4.25 \mathrm{~mm}$ radius bubble rising through a $164 \mathrm{~m}$ water column, modelled using results from McGinnis et al. (2006). 


\subsection{Seep distribution along the Hikurangi Margin}

\subsubsection{Interpreting determined seep locations}

We have used regional summed-intensity maps of the survey area to identify 585-660 seeps imaged on the TAN1404 voyage and 236-271 seeps imaged on the TAN1505 voyage (mapped in Figure 4.11). The concentration of these seep is also mapped in Figure 4.12 and Figure 4.13 for seeps imaged on TAN1404 and TAN1505 respectively. There is a general agreement in areas where seeps are most concentrated between the datasets initially collected on TAN1404 and resurveyed on TAN1505. Within these survey areas, there do however seem to be some differences between individual seep locations for the two voyages. These discrepancies in identified seep locations may be partly due to the ephemeral nature of the seeps, where some may have not been active when resurveyed on the TAN1505 voyage. It is also noticeable in Figure 4.11 that lines of imaged flares seem to align with the travel direction of the ship. Correlations between ship direction and identified seep locations imply that discrepancies in imaged seep locations may also be due to partial imaging of the water column (detailed in Section 2.3). Parts of the watercolumn above the swath remain un-imaged due to the triangular shape of echograms, which results in fragmented imaging of flares in the outer part of the swath. Partially imaged flares are displayed as lesser summedamplitude seep indicators and are often overlooked in total seep counts. Fragmented imaging of flares may explain the discrepancies of flare location between voyages and also suggests that total seep counts we have made are minimum estimates.

To compare the distribution of Hikurangi Margin seeps to other published seep fields, we have selected a section of the surveyed area that we have deemed to have a seep concentration representative of the seep field. The area defined as the seep field is based on the distribution of discovered flares in Figure 4.11 and where they are most concentrated (using information from Figure 4.11, Figure 4.12 and Figure 4.13). In comparison to other published seep field surveys (see Table 4.2), the seeps along the Hikurangi Margin are distributed more sparsely over a larger area $\left(\sim 89.57 \mathrm{~km}^{2}\right)$. Figure 4.12 and Figure 4.13 show that maximum seep concentrations reach $\sim 21$ seeps per $\mathrm{km}^{2}$. The total number of flares in the Hikurangi Margin seep field is much larger than all other published seep field results. The comparatively high number of flares that are distributed over a large area of the Hikurangi Margin suggests that underlying gas reservoirs and migration pathways are more widely spread in comparison to those below other seep fields recorded in Table 3.1. 


\begin{tabular}{|c|c|c|c|c|}
\hline Location & $\begin{array}{l}\text { Number of } \\
\text { Inidividual } \\
\text { Seeps }\end{array}$ & $\begin{array}{l}\text { Size of Seep } \\
\text { Area }\left(\mathrm{km}^{2}\right)\end{array}$ & $\begin{array}{l}\text { Number of } \\
\text { seeps per } \mathrm{km}^{2}\end{array}$ & Source(s) \\
\hline Kattegat & $\sim 100$ & $3 \times 10^{4}$ & $\sim 4 \times 10^{-2}$ & $\begin{array}{l}\text { Dando and } \\
\text { others } 1994\end{array}$ \\
\hline $\begin{array}{l}\text { Tommeliten, } \\
\text { Norwegian } \\
\text { North Sea }\end{array}$ & 120 & $6.5 \times 10^{-3}$ & $1.85 \times 10^{5}$ & $\begin{array}{l}\text { Recalculated } \\
\text { from Hovland } \\
\text { and Judd } 1988\end{array}$ \\
\hline $\begin{array}{l}\text { Torry Bay, } \\
\text { Scotland }\end{array}$ & $70-100$ & $2.4 \times 10^{-3}$ & $4.17 \times 10^{5}$ & $\begin{array}{l}\text { Judd and others } \\
2002\end{array}$ \\
\hline $\begin{array}{l}\text { Golden Sands, } \\
\text { Bulgarian Black } \\
\text { Sea }\end{array}$ & 1200 & 0.3 & $4 \times 10^{4}$ & Dimitrov 2002 \\
\hline $\begin{array}{l}\text { Golden Sands } \\
\text { NE, } \\
\text { Bulgarian Black } \\
\text { Sea }\end{array}$ & 200 & 0.0125 & $1.6 \times 10^{5}$ & Dimitrov 2002 \\
\hline $\begin{array}{l}\text { Zelenka, } \\
\text { Bulgarian Black } \\
\text { Sea }\end{array}$ & 800 & 0.33 & $2.42 \times 10^{4}$ & Dimitrov 2002 \\
\hline $\begin{array}{l}\text { Santa Barbara } \\
\text { Channel, } \\
\text { California, USA }\end{array}$ & $>500$ & $3 \times 10^{-3}$ & $>1.67 \times 10^{6}$ & $\begin{array}{l}\text { Hornifus and } \\
\text { others } 1999\end{array}$ \\
\hline $\begin{array}{l}\text { Northern } \\
\text { Hikurangi } \\
\text { Margin Seep } \\
\text { Field, } \\
\text { New Zealand }\end{array}$ & $495-567$ & 89.57 & $5.5-6.1$ & This thesis \\
\hline
\end{tabular}

Table 4.2: Seep field evaluations from other globally documented studies (Dando et al., 1994; Judd and Hovland, 1992; Hornafius et al., 1999; Dimitrov, 2002), compiled by Judd (2004), compared to seep field evaluations from this thesis. 
At the time of discovery of the seeps analysed in this thesis, there were only 36 documented flares along the Hikurangi Margin (Greinert et al., 2010) distributed throughout seep fields mapped in Figure 4.11. These seep fields are also well spaced, which supports the premise that gas is escaping from widespread underlying methane sources. It is expected that the margin accommodated large, widespread reservoirs of methane, with first-order estimates of $24 \mathrm{~m}^{3}$ of fluid per meter of strike per year injected into the margin (Townend, 1997). Widespread gas release across the Hikurangi Margin suggests there are likely many other undiscovered seeps across the continental slope. These uncharted seeps are most likely well distributed as had been observed by (Greinert et al., 2010) and this thesis. As there is a plentiful supply of methane and an accommodating tectonic system for methane transportation, it is possible that the undiscovered seeps are of a considerable quantity. 


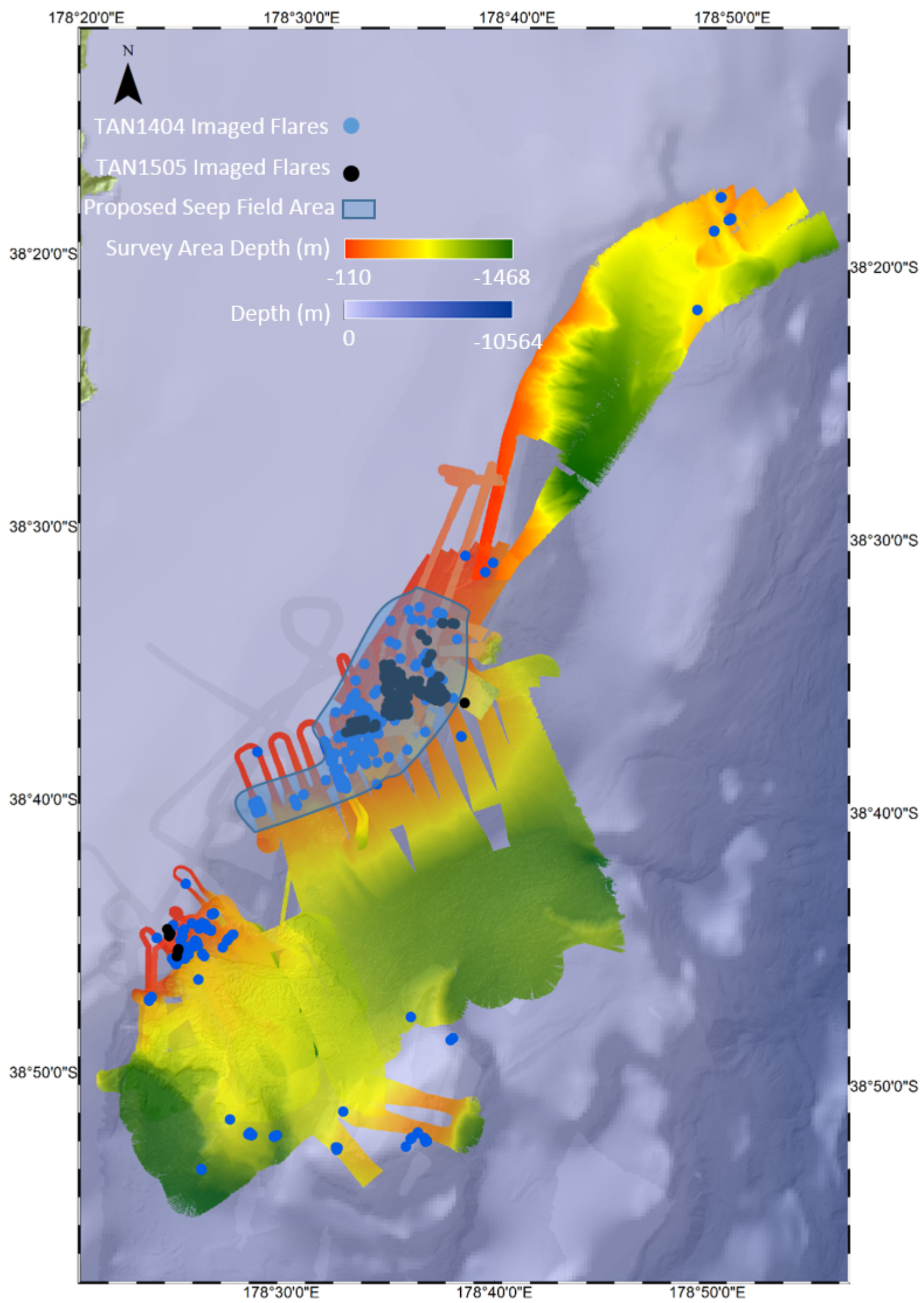

Figure 4.11: Seep locations identified using summed-intensity surfaces as in Figure 3.3. Flares imaged during TAN1404 and TAN1505 are plotted as red and black circles respectively. We have also selected an area from the survey region that is densely populated with seeps, assumed to be a seep field. 

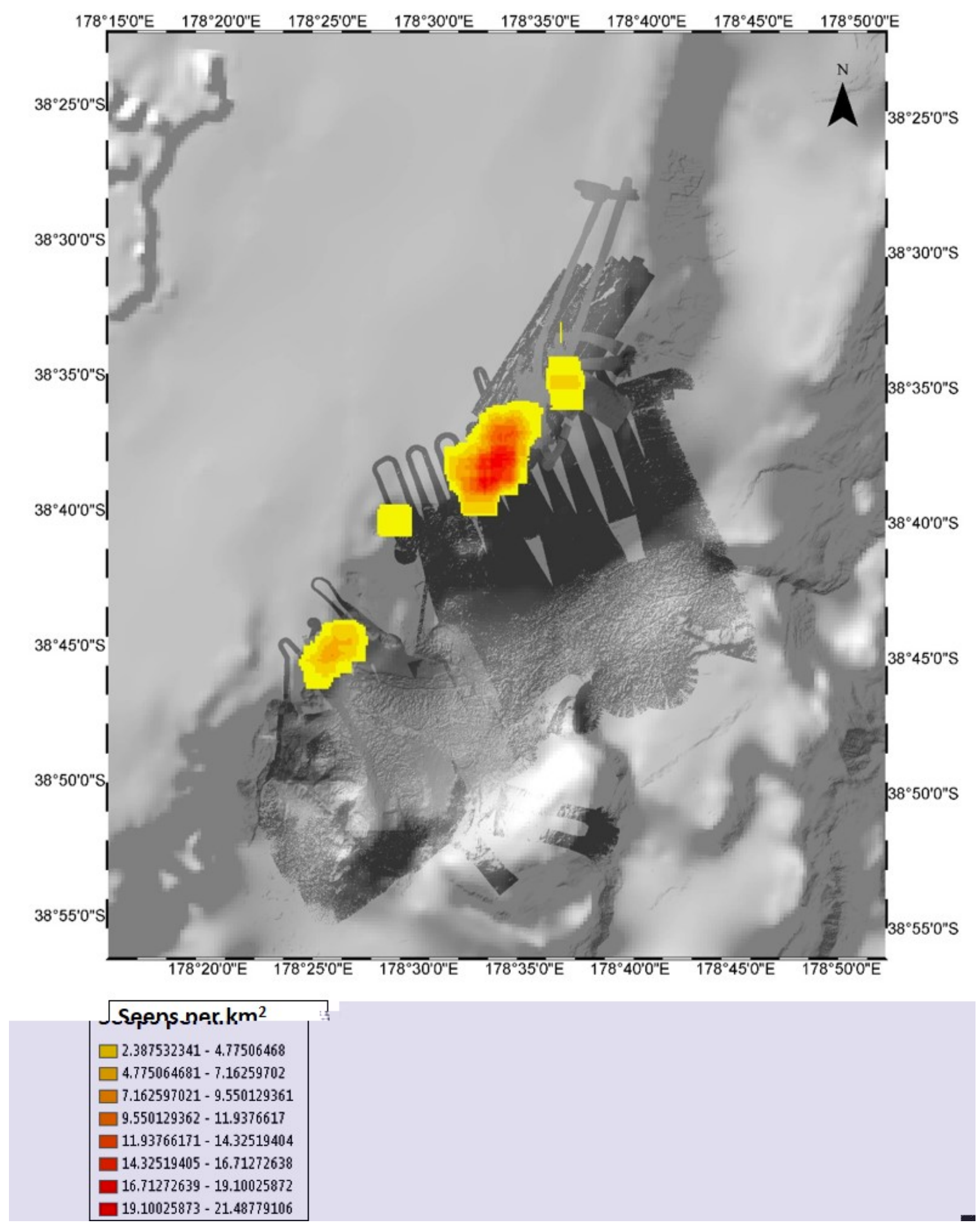

Figure 4.12: Concentration of imaged seeps per square kilometer surveyed during TAN1404. 


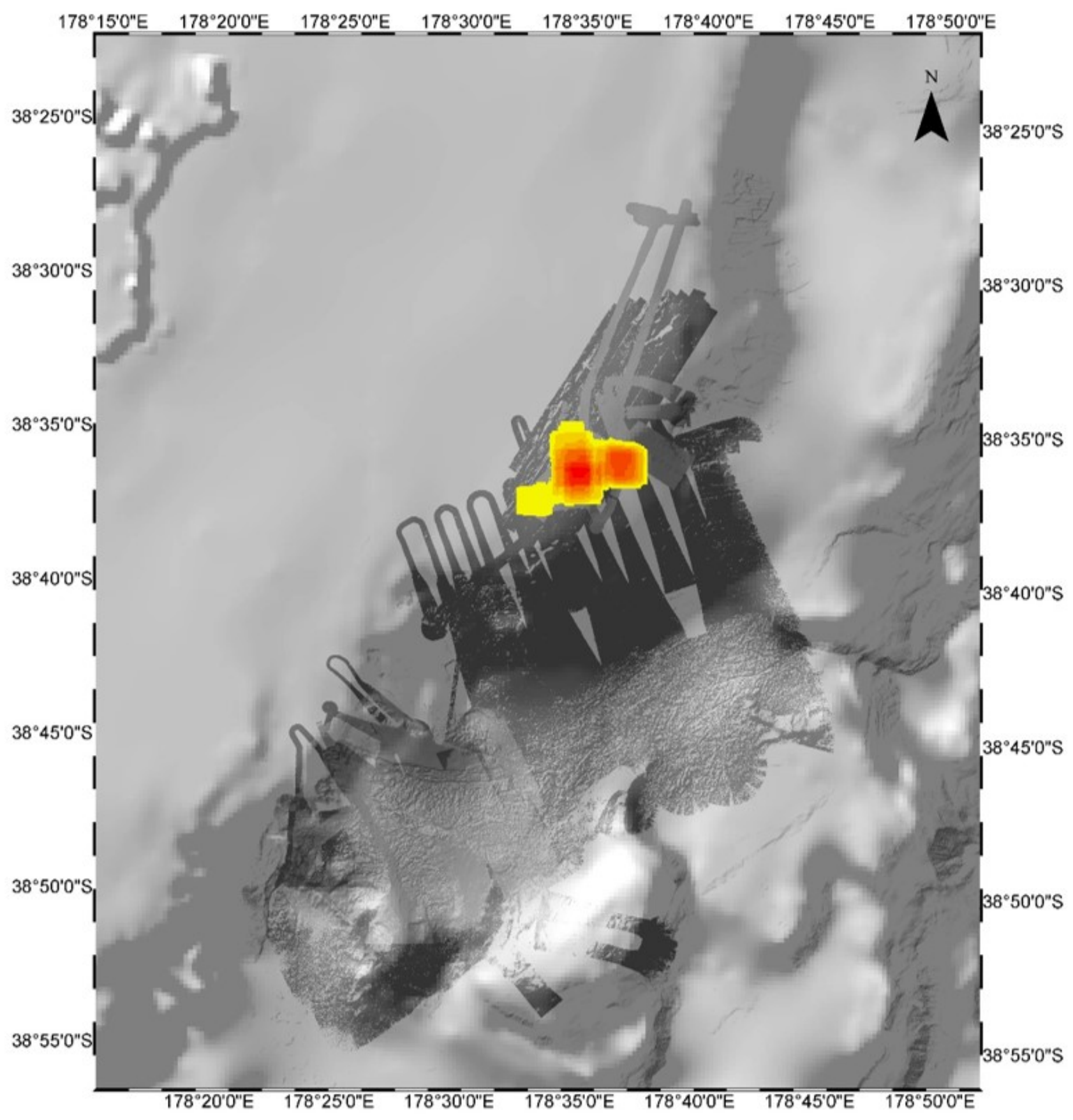

\begin{tabular}{|l|}
\hline Seeps per $\mathbf{k m}^{2}$ \\
$\square 2.414308973-4.828617944$ \\
$\square 4.828617945-7.242926915$ \\
$\square 7.242926916-9.657235887$ \\
$\square .657235888-12.07154486$ \\
$\square 12.07154487-14.48585383$ \\
$14.48585384-16.9001628$ \\
$16.90016281-19.31447177$ \\
$19.31447178-21.72878075$ \\
\hline
\end{tabular}

Figure 4.13: Concentration of imaged seeps per square kilometer surveyed during TAN1505. 


\subsubsection{Estimations of regional seafloor methane flux}

As noted in Section 4.3, the majority of analysed seeps emit fluids in the range of $\sim$ $37-286 \mathrm{~mL} / \mathrm{min}$. Conversely, there is evidence that higher-energy seeps are capable of producing fluids at rates of $\sim 1115-3371 \mathrm{~mL} / \mathrm{min}$. To extrapolate methane fluxes over the area of the Hikurangi Margin, we have approximated the proportion of other highenergy flares within the survey region that haven't been recorded in the singlebeam. Using the regional summed intensity maps (Figure 3.3) we have recognised that flare signatures larger than $25 \mathrm{~m}$ diameter seem to correspond with more prominent flares in stacked curtain sections (as in Figure 1.1).

Based on these assumptions, 5-7\% of the seeps surveyed on TAN1404 and TAN1505 are of high-energy (where the range is due to the uncertainty in the total flare count). The total number of seeps have been taken from those imaged on TAN1404 (Table 3.1), which fully covers the area surveyed for TAN1505. From this, we have estimated, there are $\sim 40$ high-energy seeps and 545-621 seeps of typically observed methane release.

Using the seafloor estimates of seep fluids emissions from Table 4.1, we have extrapolated the proportions of typical and high-energy seep occurrence to calculate an approximation of the flux of methane released from the seafloor over the survey area. Calculations approximate $0.99 \times 10^{5} \pm 0.64 \times 10^{5} \mathrm{~m}^{3} /$ yr of methane $\left(6.43 \times 10^{7} \pm 4.21 \times\right.$ $10^{7} \mathrm{TgCH}_{4} / \mathrm{yr}$ ) is escaping from the seafloor over the surveyed region. Evidence of other seeps is currently sparse across the margin (Figure 1.5), with the recently discovery of this seep field increasing the total discovered seeps across the Hikurangi Margin by $\sim 16-18$ fold. Due to the majority of discovered seeps being situated in the recently discovered seeps field, these methane flux estimates are a close approximation for the currently discovered seeps across the Hikurangi Margin.

\subsection{Implication for global seep models}

The quantity of seep-produced methane that is escaping from the seafloor over the scale of entire seep fields and globally is still poorly constrained. This is primarily due to the limited coverage of seep activity over these large scales (e.g. entire subduction margins). Seep locations are both difficult to predict and subject to change over ephemeral timescales (Sibuet et al., 1988). Mapping large seep fields has also only become realistic in the last decade with the added capabilities of watercolum-imaging for multibeam echosounders (Clarke, 2006). 
Due to limited knowledge the global distribution of active seeps, atmospheric greenhouse emissions attributed to submarine seeps are even more poorly understood. No accurate means of quantifying seep-produced atmospheric methane emissions has been established. As a results, methane emissions from marine geological sources have not been included in previous Intergovernmental Panel on Climate Change's assessment reports.

Development of comparatively high accuracy seep analysis tools in this thesis will hopefully progress seep emission research further towards a stage where accurate global seep emissions can be made. Making reasonable estimations of global seep-produced atmospheric methane emissions is becoming increasing important, as research has shown that the globally summed emissions could be considerable.

\subsection{Correlations between seep emission flux and summed-acoustic intensity}

Although singlebeam data have proven to be effective in quantifying individual seepfluid fluxes, singlebeam echosounders are not commonly used for purposes other than fish stock analysis. As fish-stock assessment usually repeats surveys over similar areas, global singlebeam coverage is sparse but temporally repetitive. In global seep-fluid output models, large gaps in singlebeam coverage are accounted for by extraploting data from surveyed seep fields. These extrapolations are the cause for such high uncertainties and variations in previously published global estimates (Trotsyuk and Avilov, 1988; Lacroix, 1993; Hornafius et al., 1999; Ehhalt and Schmidt, 1978; Hovland et al., 1993).

Much of the extrapolation-caused error would be drastically reduced if the use of singlebeam-sampled flare profiles could be replaced by multibeam-sampled flare profiles. There is extensive global coverage of multibeam surveys, because of its application of mapping bathymetry. Within the last decade, most multibeam systems are capable of acoustically-imaging the water column as well. Globally widespread multibeam coverage implies that a large number of seeps have been acoustically imaged with multibeam systems; however there is presently no means to approximate seep fluid flow from these data alone. We note that these methods still under sample the watercolumn,

Currently, water column imaging systems are not correctly calibrated for quantified analysis of seep-bubble echoes. When an object is imaged using a singlebeam 
echosounder, the acoustic energy reflected back from that object is inherent from only one incident sonar beam. The ratio of the measured signal strength of the received pulse to the emitted pulse is directly related to the amount of scattering caused by the object. The amount of scattering can then be correlated to the size of the object. In the case of a multibeam imaged object, an object echoes energy from multiple incident sonar beams. These beams arrive at the object at different angles and times. The complex echoing of energy from multibeam imaged object makes it difficult to reconstruct the exact scattering signature of each imaged object. Due to these limitations, measuring the sizes of a collection of bubbles is not yet possible with multibeam echosounders.

In order to test whether a relationship exist between mutibeam acoustic returns and calculated seep-fluid flux, we have tested correlations between these data sets, we have first tested the dependence of mulitibeam seep-bubble returns on the speed that the ship is travelling. For this, a single seep (imaged as Flare F in Figure 3.16) was surveyed using the multibeam over four transects (Figure 4.14) within the space of an hour at different ship speeds. Figure 4.15 displays histograms of summed intensity (produced using Summed_Intensity_Maps.m) derived from multibeam data collected on each pass. Each histogram has been plotted against the speed of the boat as it passed over the seep. It is difficult to derive a relationship between summed intensity and ship speed from Figure 4.15 as the strength of seep activity may also be changing between each survey line. It seems from Figure 4.15 that instantaneous changes in seep fluid release have more of an impact on summed intensity than changes in boat speed. Due to this, we have chosen not to include the influence of boat speed on the relationship between summed intensity and seep-fluid flux in further relationship tests.

Summed-intensity histograms Figure 4.16 have been plotted again for flares F1, F2 and F3 (surveyed on lines 10, 14 and 22 respectively) that were uninhibited by sonar noise (from either fish or plankton) in Figure 4.16. These summed-intensity histograms have been plotted against seep-fluid flux measurements, calculated for each pass over the seep. It is apparent from Figure 4.16 that the area under the histograms increases with increasing flux measurements. A larger area under the plot implies that a higher number of echoes were recorded and hence a higher number of bubbles were identified in the seep. The observed relationship between summed-intensity values and seep-fluid flux measurements suggest that a correlation does exist.

Presently, any correlations between multibeam imaged seeps and seep-fluid flux are relative and not quantifiable. It is likely that ship speed does influence the summedintensity values of flares; however this relationship has not been quantified in this thesis due to limited data. It is also noted that the summed-intensity values plotted 


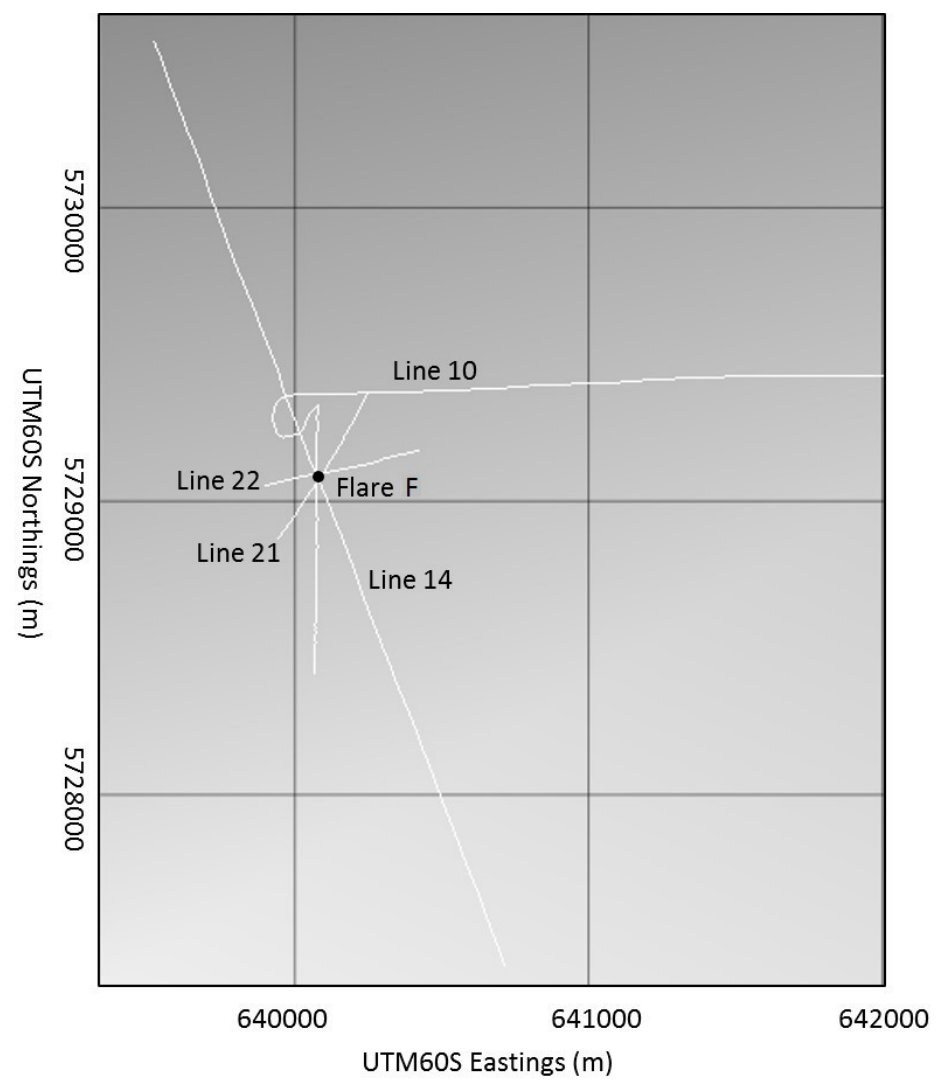

Figure 4.14: Sonar lines 10, 14, 21 and 22 from TAN1505 that acoustically surveyed Flare F (Figure 3.16)

as histograms in Figure 4.16 are considerably smaller than those plotted in Figure 3.7. The contrast in summed intensity values between flares are a result of differences in acoustic data resolution. Summed-intensity surfaces in Figure 3.6 were created from a much higher resolution volume of acoustic returns than those plotted in Figure 4.16. As the higher resolution volume is gridded more densely, more data will be vertically summed when calculating summed-intensity surfaces. Therefore, the final summedintensity values are directly related to the initial data-resolution of the water-column imaged volume.

Based on this information, we suggest that summed-intensity values of flares are dependent on ship speed, data resolution and the flux of ensuing seep fluid. This relationship indicates that estimating seep-fluid flux from multibeam imaged seeps may become possible once these other dependencies have been quantified. 

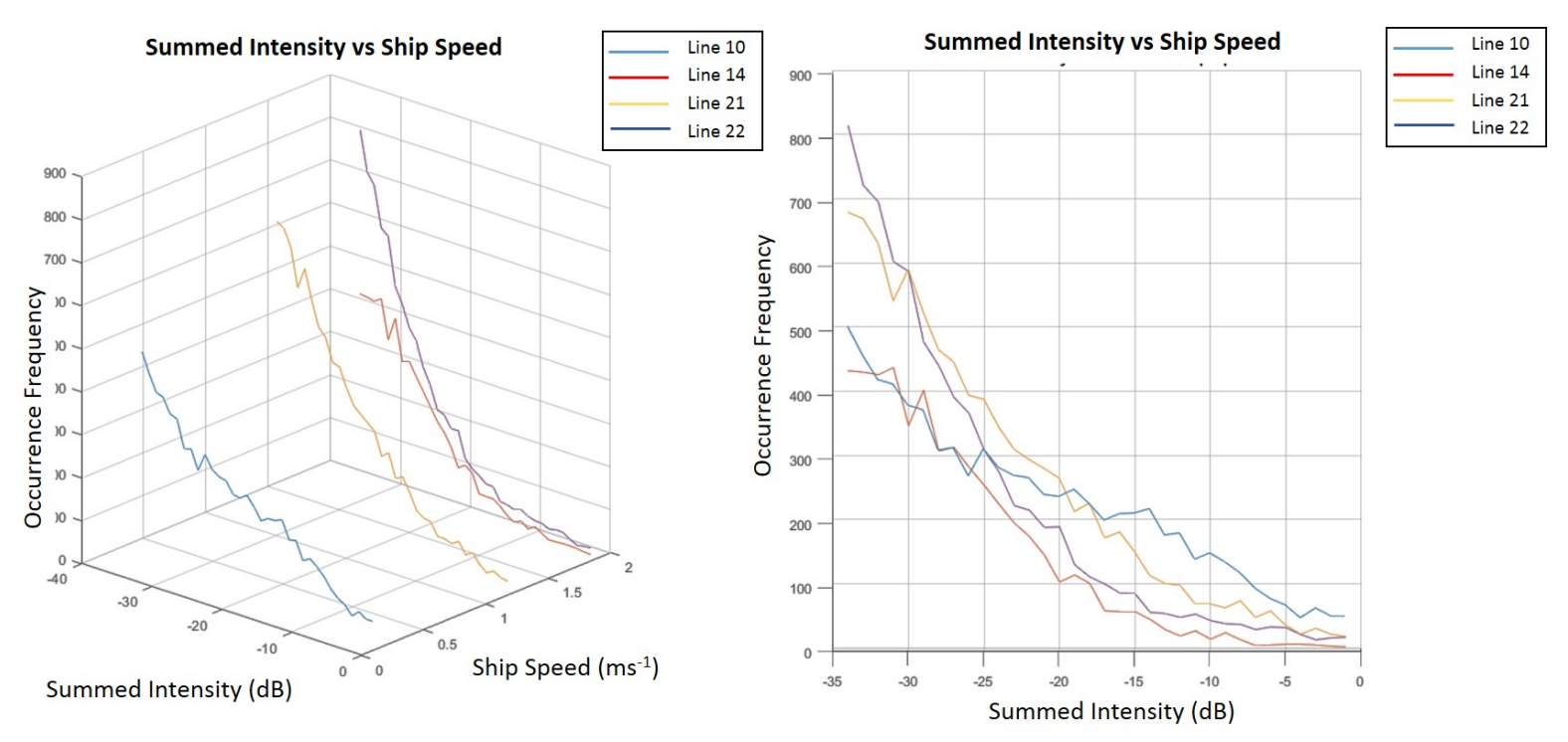

Figure 4.15: Plot of summed-intensity histograms for Flare F against the speed of the ship for each pass.
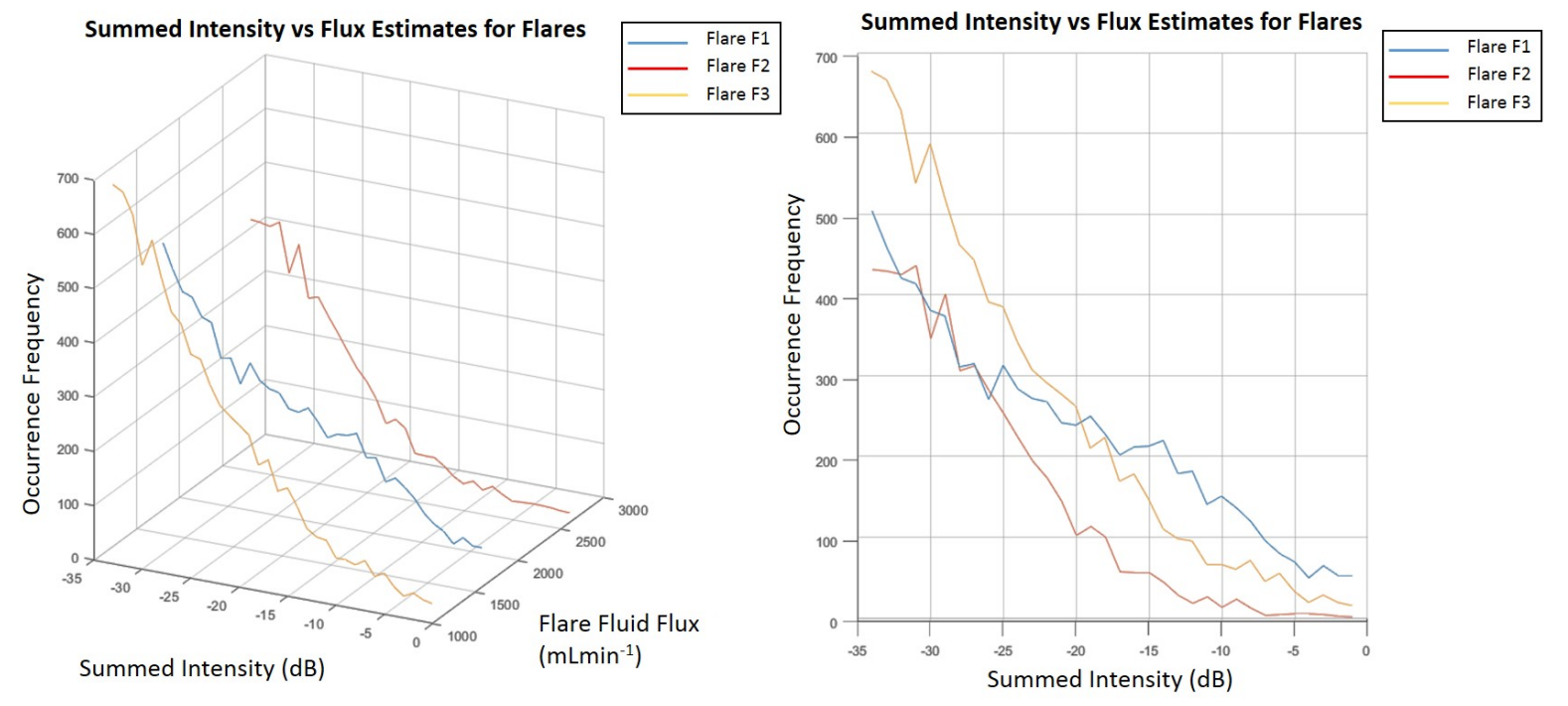

Figure 4.16: Plot of summed-intensity histograms for Flare F against the seep-fluid flux at the seafloor for each pass. 


\subsection{Future work}

Bubble-size distributions measured in this thesis are limited to a narrower range of sizes $(\sim 6-15 \mathrm{~mm})$ than other previously published measurements $(\sim 1-20 \mathrm{~mm})$. We have inferred that the absence of smaller and larger bubbles is due to video quality and an inadequate recording time, respectively. To better constrain bubble sizes outside of our observed range, it would be useful to revise our measurements with higher-resolution camera footage over a longer observation period.

We have made approximations of the degree to which theoretical dirty-bubble models overestimate bubble-rise rates that has arisen from the slowing effect of bubble oscillations (see Section 4.2.2) that has not been previously accounted for. If more bubbles were tracked using the Matlab codes (Section 6.3 - 6.11) it would be possible to construct a more accurate trend for measured rise rates of different bubbles sizes. With bubble-rise rate measurements in the order of $\sim 100$ it would be possible to make a closer estimate of the true degree of slowed bubble ascent caused by bubble oscillations.

Throughout this thesis there have been multiple references to how seep bubble preservation increases when larger numbers of bubbles rise in close proximity. This information is very useful in inferring seeps that are potentially capable of transporting undissolved methane to the atmosphere. However, a quantified increase in bubble preservation as a function of proximal bubbles has not been established, as rising seep bubbles rise chaotically and are difficult to constrain. If first-order relationships where developed between bubble concentration at a part of a flare and methane preservation, then the outwards transfer-rate of methane from bubbles could be better constrained for a segment of a flare. This information could then be used to more-accurately predict the proportion of methane that remained in seep bubbles at shallower water depths. It would then be possible to make more accurate estimations of atmospheric methane emissions simply based on acoustic imaging.

Due to the ephemeral nature of seeps and partial imaging of the water column, the total number of flares within our survey area are only minimum estimates. To construct a more accurate impression of the seep distribution in and around the Northern Hikurangi Margin, the region would have to be repeatedly acoustically imaged with dense coverage.

As mentioned, new advancements in seep-fluid output quantifications will come with the calibration of multibeam watercolumn imaging. There is already motivation for 
this to be calculated for other watercolumn studies and development is currently ongoing (A. Pallentin \& J.M. Augustin, personal communication). If both the watercolumn calibration and methane dissolution rate quantifications were achieved, seep-related atmospheric emission rates could be calculated essentially from multibeam watercolumn imaging. Knowledge of bubble-size and rise-rate distributions would also be required (obtainable using the techniques developed in this thesis), but these are expected to be similar across an entire seep field. As a result, assessments of changes in seep-fluid output could be made of altering seep fields. This would also drastically decrease the uncertainty in current extrapolated seep-outputs over large regions. 


\section{Conclusions}

The numerous approaches used to estimate the rate at which global seep systems transfer methane into the water column are varied and involve large uncertainties. Despite these variabilities, all estimations are in agreement that the flux of methane into global oceans is substantial (estimates by Judd (2000) are in the order of $0.4-48 \mathrm{Tg} / \mathrm{yr}$ ). The amount of methane that is transported through the water column via rising seep bubbles and released into the atmosphere is even more poorly constrained, where only crude approximations have been made. In order to better constrain uncertainties in methane budgets transported to the water column and atmosphere, better tools and techniques for modelling parameters of rising seep bubbles are required.

The techniques that we have developed in this thesis are used to calculate a series of seep parameters to better constrain seafloor methane flux estimates. These techniques comprise of (1) methods of acquiring bubble size and rise rate distributions from underwater camera footage of rising seep bubbles, (2) mapping the distribution of seep locations within multibeam surveyed regions, and (3) producing profiles of acoustically imaged flare intensity at staggered depth intervals for assessing the preservation of rising seep bubbles in the water column.

These methods have been applied to a recently discovered abundant seep field across the raised continental slope of the Hikurangi Margin, New Zealand. Seeps discovered along the upper Margin are situated at uncharacteristically shallow depths $(\sim 150-$ $300 \mathrm{~m} \mathrm{bsl}$ ), which implies the methane released at the seafloor via seeps may be capable of transferring to the atmosphere. Evidence of carbonate mounds at the base of active seeps has also been recorded in the bathymetry and underwater video footage for multiple seeps in the area. The presence of these mounds indicate that seeps have been active for timescales of the order of a few thousand years.

Mapped seep distributions have revealed that these recently discovered seeps are numerous and widespread in comparison to previously documented seep fields. In the total expanse of the survey area $\left(840 \mathrm{~km}^{2}\right)$ between 585 and 660 seeps were identified. A segment of the surveyed area that we defined as a seep field (due to its high concentration of seeps) contained between 495 and 567 seeps over an area of $89.6 \mathrm{~km}^{2}$ 
Techniques developed for calculating sizes and rise rates of seep bubbles were developed using videogrammetric methods. These techniques are automated in Matlab codes and can be applied to any underwater video footage that has parallel scaling lasers. Implementing these bubble-measurement codes on seep-bubble footage from the Hikurangi Margin has produced distributions of bubble sizes and rise rates that were compared to previously published seep bubble distributions. Experimental bubble-size measurements were in general agreement with previously published bubble-size results. Conversely, we were unable to obtain experimental results for bubble-rise rates from literature inquiries. Experimental bubble-rise rate distributions we have obtained are compared to theoretical bubble-rise rate models, which predict consistently faster rising seep bubbles than were observed. Overestimations in theoretical bubble-rise velocity predictions are likely due to assumptions that rising dirty bubbles (bubbles with a coating of surfactants) behave as solid spheres. Elliptically shaped bubbles oscillate when rising, and whilst the presence of surfactants acts to dampen bubble oscillations, they are not eliminated completely. Completely neglecting bubble oscillations in dirtybubble rise rate models fails to account for the extra energy losses to the rise of the bubble, hence overestimates rise velocity. By shifting the theoretical models, we were able to approximate the degree of unaccounted slowing that bubble oscillations might cause. In the case of Leifer et al. (2000), overestimations ranged from $3-3.7 \mathrm{~cm} / \mathrm{s}$, although more bubble rise-rate measurements could make this estimation more precise. Shifted theoretical bubble-rise rates were used as distributions representative of the experimentally measured bubble-rise rates.

Improved methods for calculating bubble-size and rise-rate distributions were then combined with the FlareFlow module developed by Mario Veloso to obtain methane fluxes of seeps. Flux calculations for seeps across the newly discovered Hikurangi Margin area, revealed that standard seeps release between 37 and $286 \mathrm{~mL} / \mathrm{min}$ of undissolved methane. There is also evidence for more prominent seeps within the survey area that has the capacity to release between of undissolved methane in the range of 1115 to $3371 \mathrm{~mL} / \mathrm{min}$.

We have applied models of methane preservation in rising seep bubbles to a clearly imaged flare that appeared to extend the full height of the water column. Combining chemical dissolution models with staggered-depth intensity models has shown that the majority of methane in bubbles is lost within the first $60 \mathrm{~m}(\sim 100 \mathrm{~m} \mathrm{bsl})$. We have approximated the proportion of the initial seafloor-released methane that is preserved to the atmosphere to be in the order of $0.2 \%$.

Development of these techniques will hopefully contribute in progressing seep research to a standard where global emissions can be more accurately estimated. Before these 
estimations are plausible, we suggest that multibeam water-column imaging must first be calibrated, so that assessing outputs of individual seeps across extensive seep fields becomes realistic. 


\section{Appendix}

\subsection{Summed_Intensity_Maps}

$\%$ produces an integrated intensity sufrace for point data exported $\%$ from FMMidwater

$\%$ clear the workspace

close all

clear all

$\%$ enter collumn height in metres

for column_height $=10000$;

\%enter the thickness of the soundfront error to be ignored floor_height $=20$;

$\%$ choose the numer of grid squares in the latitudinal direction of the final $\%$ mosaic

num_squares $=200$;

$\%$ run the module 'ascii_columns' to load in the data ascii_columns

$\%$ variables that will be used to store the binned data

$\mathrm{x}_{-} \mathrm{bin}=0$;

$\mathrm{y}_{-} \mathrm{bin}=0$;

$z_{-}$bin $=0$;

a_bin=0;

$\%$ compile a matrix made up of $\mathrm{x}, \mathrm{y}, \mathrm{z}$, amp

data_comp $=$ [samp_pos_x, samp_pos_y, samp_pos_z, samp_val_amp] ;

$\%$ make an horizontal grid spacing

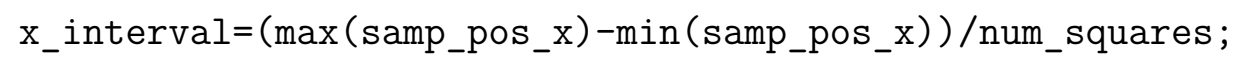

$y_{\text {_interval }}=(\max ($ samp_pos_y $)-\min ($ samp_pos_y $)) /$ num_squares ; 
$\%$ the number of grid squares in the longitudinal direction is evaluated so $\%$ that all grid spaces are square

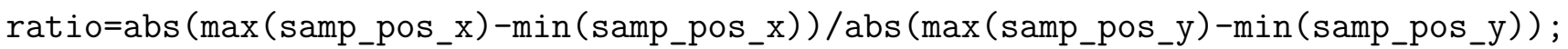

num_y=round (num_squares/ratio);

$\%$ for all grid spaces in the latitunidal direction

for $s q=1$ :num_squares

$\%$ display sq as a progress counter

$\operatorname{disp}(\mathrm{sq})$

\%takes data values for all the columns that are within the latitude range $\%$ of the current grid square

for rows $1=1:$ length (data_comp $(1,:)$ )

between_x $(:, r o w s 1)=$ data_comp $\left(\right.$ data_comp $(:, 1)>\left(\min \left(\operatorname{samp} \_p o s_{-} \mathrm{x}\right)+(\right.$ sq-1) $* \ldots$

$x_{-}$interval) \& data_comp $(:, 1)<\left(\min \left(\operatorname{samp}_{-}\right.\right.$pos_x $)+$sq*x_interval), rows 1$)$;

end

$\%$ if no values are present in the $\mathrm{x}$ range, the column values are 0,0,0,0 for $\%$ that grid square

if isempty(between_x);

between_x $=[0,0,0,0]$;

end

$\%$ for all grid spaces in the longitunidal direction

for sq2=1:num_y

$\%$ takes data values for all the columns that are within the longitude range

$\%$ of the current grid square

for rows $2=1:$ length (data_comp $(1,:)$ )

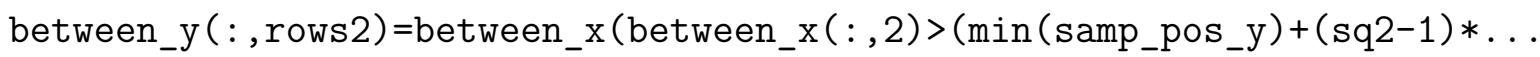

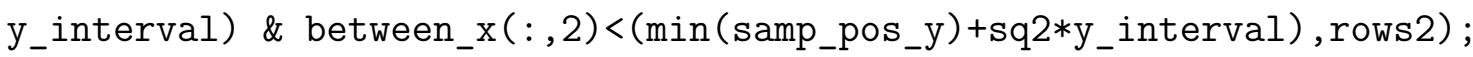

end

$\%$ if no values are present in the y range, the column values are 0,0,0,0 for $\%$ that grid square

if isempty(between_y);

between_y=[0,0,0,0] ;

end 
$\%$ take data values between the sepcific vertical integration range between_col=between_y (between_y $(:, 3)>\left(\min \left(\operatorname{between} y(:, 3)+f l o o r \_h e i g h t\right)\right)$ \& between_y $(:, 3)<\ldots$

(min (between_y $(:, 3)+$ column_height)) , : ) ;

$\%$ if no values are present in the vertical range, the column values are 0,0,0,0 $\%$ for that grid square

if isempty(between_col);

between_col=[0,0,0,0];

end

$\%$ records the binned values in seperate arrays

$\mathrm{x}_{-}$bin (length (x_bin $\left.(:, 1)\right)+1$ : length $\left(\mathrm{x}_{-} \mathrm{bin}(:, 1)\right)+\ldots$

length (between_col $(:, 1)), 1)=\operatorname{between} \operatorname{col}(:, 1)$;

y_bin (length (y_bin $(:, 1))+1:$ length (y_bin $(:, 1))+\ldots$

length (between_col $(:, 1)), 1)=\operatorname{between} \operatorname{col}(:, 2)$;

$z_{-}$bin (length (z_bin $\left.(:, 1)\right)+1:$ length $\left(z_{-}\right.$bin $\left.(:, 1)\right)+\ldots$

length (between_col $(:, 1)), 1)=$ between_col $(:, 3)$;

a_bin (length (a_bin $(:, 1))+1:$ length (a_bin $(:, 1))+\ldots$

length (between_col $(:, 1)), 1)=\operatorname{between} \operatorname{col}(:, 4)$;

$\%$ clear the values for the next iteration

between_y=[];

end

between_x=[];

end

end

$\%$ combine seperate arrays into a matrix

flare $=\left[y_{-}\right.$bin, x_bin, z_bin, a_bin] ;

$\%$ cut out rows that only contain zeros

flare $(f \operatorname{lare}(:, 4)==0,:)=[]$;

\%writes data to a file containing [lat, lon, depth, amplitude] that can be $\%$ loaded as a surface back into Fledermaus

dlmwrite('newflare.txt', flare, 'delimiter' , '\t', . .

'precision', , \%.6f'); 


\section{2 ascii_columns}

\%imports water column point data from FMMidwater

$\%$ choose the text file

filename = uigetfile('*.txt');

data $=$ dlmread $($ filename, ' ', 14,0);

\%assign values to each column

samp_file_id=data $(:, 1)$;

samp_pos_x=data $(:, 4)$;

samp_pos_y=data $(:, 6)$;

samp_pos_z=data $(:, 8)+\operatorname{data}(:, 9)$;

$\%$ select the required range of values values

dataedit=data $(:,[11: 16])$;

dataedit (dataedit $>0)=0$;

samp_val_amp=sum (dataedit, 2);

\subsection{DTIS Frame Dimensions}

\%Core program for calculating running all modules and writting BSD .txt files

$\%$ clear the existing variables and figures

close all

clear all

$\%$ Allow the user to choose a video file for analysis

bubbleVideo $=$ uigetfile('*', 'Select the video for analysis');

workingDir = tempname;

mkdir (workingDir)

mkdir (workingDir, 'images')

hVideoSrc = vision.VideoFileReader(bubbleVideo, 'ImageColorSpace', 'RGB'); vid=VideoReader (bubbleVideo);

$\%$ choose how many frames to skip in each iteration frame_count $=5$; 
$\%$ create an empty matrix to enter bubble-centre coordinatese

$\%$ (coordinates $(\mathrm{x}, \mathrm{y})$, bubble number, iteration_number)

bubble_centre=zeros $(2,100,100)$;

$\%$ (iteration number, bubble number)

bubble_height=zeros $(100,100)$;

$\%$ (iteration number, bubble number)

bubble_radius $=$ zeros $(100,100)$;

$\%$ run the module 'staring_point' that allows the user to specify the frame $\%$ of the loaded video

starting_point

$\%$ count of what frame iteration the program is on

iteration=1;

$\%$ value that is subject to change depending on whether the user wishes to

$\%$ repeat a step

repeat $=1$;

$\%$ loop where each cycle is a frame iteration

while isDone(hVideoSrc) \&\& repeat == 1;

$\%$ clear repeat for each loop

clear repeat

$\%$ clear all other variables except those that are needed for further iterations clearvars -except bubbleVideo filename hVideoSrc workingDir...

bubble_centre frame frame_count iteration bubble_height frame_number...

bubble_radius imgA

$\%$ close all figures from the previous iteration

close all

$\%$ run the module 'pick_image' that allows the user pick points on the chosen image

pick_image

$\%$ run the module 'canadian_grid_lasers' that allows the user to specify the $\%$ frame of the loaded video

canadian_grid_lasers

$\%$ run the module 'canadian_grid_measure' constructs a Canadian Grid for the $\%$ frame 
canadian_grid_measure

$\%$ run the module 'bubble_size_and_pos' that measures the size and elevation $\%$ of bubbles

bubble_size_and_pos

\%ask the user if they have finished working on this and would like to move $\%$ on to the next slide

disp('would you like to go to the next slide?');

$\%$ the user must enter ' 1 ' to move on, or ' 0 ' to remain on this slide

repeat=input('enter 1 for yes or 0 for no: ');

$\%$ after moving on, the iteration increases by +1

iteration=iteration +1 ;

\%The video time of each frame is calculated using:

run_time=frame_number/bubbleVideo. FrameRate;

$\%$ take BSD values above 0, convert from $\mathrm{cm}$ to $\mathrm{m}$, then round to the nearest $\% 0.00125$ (as is a standard binnig size for BSD (FlareFLow User Manuel) bsd=round ( (bubble_radius (iteration- . .

$1,1:$ length (bubble_radius (bubble_radius (iteration-1, : ) > . .

0)))/100)/0.00025)*0.00025;

$\%$ create an empty column for normalized frequency

$\operatorname{bsd}(2,:)=$ zeros ;

$\%$ add rows for maximum and minimum bubble sizes

$\%$ convert from $\mathrm{cm}$ to $\mathrm{m}$

$\operatorname{bsd}(3,:)=$ bubble_radius_max_unc (iteration- ...

1,1:length(bubble_radius_max_unc(bubble_radius_max_unc(iteration-1,: )>0))) /100;

$\%$ create a column for minimum uncertainty values

$\operatorname{bsd}(4,:)=$ bubble_radius_min_unc (iteration- ...

$1,1:$ length (bubble_radius_min_unc(bubble_radius_min_unc (iteration-1,: )>0))) $/ 100$;

\%make empty arrays for incorporated max and min distribution errors $\operatorname{bsd}(5,:)=\operatorname{zeros}(1$, length $(\operatorname{bsd}(1,:))) ; \operatorname{bsd}(6,:)=\operatorname{zeros}(1, \operatorname{length}(\operatorname{bsd}(1,:)))$;

$\%$ sort the bsd matrix in order or bubble size

[sort1, $\operatorname{sort} 2]=\operatorname{sort}(\operatorname{bsd}(1,:))$;

$\mathrm{bsd}=\mathrm{bsd}(\mathrm{:}$, sort 2$)$; 
\%normalise the occurence frequcy of each bubble-size bin and populate bsd(2,:) for $n=1:$ length (bsd $(1,:))$

if $\operatorname{sum}(\operatorname{bsd}(1, n)==\operatorname{bsd}(1,1: n-1))==0$;

$\%$ give each binned bubble size an occurrence frequency.

$\%$ repeated numbers are given a 0

$\operatorname{bsd}(2, \mathrm{n})=\operatorname{sum}((\operatorname{bsd}(1, \mathrm{n})==\operatorname{bsd}(1,:)>0))$;

end

end

$\%$ inital numbers are rounded for the puroposes of binning, uncertainty \%errors are kept unrounded

for $\mathrm{n}=1:$ length (bsd $(1,:))$

$\%$ if there are repeated values

if $\operatorname{bsd}(2, n)>1$

$\%$ find the largest possible uncertainty

$\operatorname{bsd}(5, n)=\max (\operatorname{bsd}(3, \operatorname{bsd}(1,:)==\operatorname{bsd}(1, n)))$;

$\operatorname{bsd}(6, n)=\min (\operatorname{bsd}(4, \operatorname{bsd}(1,:)==\operatorname{bsd}(1, n)))$;

$\%$ if that bubble size is unique, its uncertainty stays the same

elseif $\operatorname{bsd}(2, n)==1$

$\operatorname{bsd}(5, n)=\operatorname{bsd}(3, n)$;

$\operatorname{bsd}(6, n)=\operatorname{bsd}(4, n)$;

end

end

$\%$ delete the repeats labelled with $0 \operatorname{bsd}=\operatorname{bsd}(:, \operatorname{bsd}(2,:)=0)$;

$\operatorname{bsd}(2,:)=\operatorname{bsd}(2,:) . / \max (\operatorname{bsd}(2,:))$;

$\%$ remove the orignal uncertainty rows

$\operatorname{bsd}(3: 4,:)=[]$;

$\%$ add the uncertainty factor for helicle/zigzag rise path

$\%$ this is chosen from experimental results

$\%$ results from this thesis are +/-

$\operatorname{bsd}(3,:)=\operatorname{bsd}(3,:)+0$;

$\operatorname{bsd}(4,:)=\operatorname{bsd}(4,:)-0$;

$\%$ Write a text file containing a column for - radius, normalised frequency \%Name this file by - 'movie name' _frame number' _'run time' filenm=fopen (sprintf ('bsd_\%s_FrameNum_\%d_RunTime_\%g.txt', filename,... frame_number, run_time), 'w'); 
fprintf(filenm,'

\%move forward the specified number of frames for the following itertion imgC=img ;

for frame $=1$ : frame_count

imgA=step (hVideoSrc);

frame_number=frame_number+1;

end

end

\subsection{Starting_Point}

\%progresses the video to the specific frame chosen by the user

$\%$ start at the first frame

frame_number $=1$;

$\%$ define imgA as a 'step' of the chosen video

imgA=step (hVideoSrc);

\%show a dialogue box that requests eiter a time or frame number to jump to prompt='Starting frame:', 'OR Starting time (s):';

dlg_title='Start';

num_lines=1;

answer $=$ inputdlg (prompt,dlg_title,num_lines $)$;

$\%$ if they try and enter numbers in both field, request that they only enter into one

if isempty (str2num(cell2mat (answer $(2,1))))<1$ \&\&...

isempty $(\operatorname{str} 2$ num $(\operatorname{cell} 2 \operatorname{mat}(\operatorname{answer}(1,1))))<1$

$\mathrm{h}=\operatorname{msgbox}($ 'ERROR: please only enter values in one box');

elseif str2num(cell2mat (answer $(1,1)))>1$

for frame=1: str2num (cell2mat (answer $(1,1)))-1$

imgA=step (hVideoSrc) ;

frame_number=frame_number+1;

end

$\%$ in the case of a starting time 
$\%$ calculate the number of frames to skip

elseif round(str2num(cell2mat (answer $(2,1))) *$ bubbleVideo. FrameRate) $>1$

for frame $=1$ : round (str2num (cell2mat (answer $(2,1))) *$ bubbleVideo. FrameRate) -1

img $A=\operatorname{step}(\mathrm{hVideoSrc})$;

frame_number=frame_number+1;

end

end

\section{5 pick_image}

$\%$ displays the frame so that points can be specified by the user to make $\%$ measurements

$\%$ creat a figure for displaying the frame $\mathrm{h}=\mathrm{f}$ igure;

$\%$ create a greyscale version of the frame imgB=rgb2gray (imgA);

$\%$ show the greyscale image

imshow (imgB)

$\%$ allow the user to adjust the contrast of the image when picking points disp('adjust the image contrast to highlight image features'); disp('press ENTER to continue');

imcontrast

$\%$ create a new figure for showing the RGB image $\%$ this can be used to check features of the greyscale image figure

imshow (imgA)

$\%$ make greyscale image the active window

figure (h)

$\%$ pause the sequece to allow the user to operate on the image pause (); 
$\%$ request that the user 'picks' the point on the image where the bubbles are $\%$ ensuing

disp('locate the vent origin')

$\%$ create empty arrays for the coordinates of picked image points

bubble_pos_x=[];

bubble_pos_y=[];

$\%$ plot the bubbles centres for 'picked' bubbles from the previus iteration

\%this will make it easier to follow bubbles and differentiate them from other $\%$ features

figure (h)

if iteration $>1$

hold on;

scatter (bubble_centre(1, bubble_centre $(1,:, 1)>\ldots$

0 ,iteration-1), bubble_centre $(2$, bubble_centre $(1, \ldots$

$:, 1)>0$, iteration-1), 'm');

end

$\%$ loop that allows the user to pick image points in a GUI interface

$\%$ this at the end of each loop this 'picking interface will end to

$\% a l l o w$ the user to zoom to other parts of the image

rep $=1$;

while $r e p==1$

clear rep

clear prompt

clear input

$\%$ on the grayscale figure

figure(h)

$\%$ start the GUI interface for picking the ends of each long and short axis

$\%$ for each chosen bubble

[pos_x, pos_y]=getpts;

bubble_pos_x (length (bubble_pos_x) $+1:$ length (bubble_pos_x $)+$ length $($ pos_x $)$ ) =pos_x ; bubble_pos_y (length (bubble_pos_y) $+1:$ length (bubble_pos_y) + length (pos_y)) =pos_y ;

$\%$ plot the points that have been picked

hold on 
scatter(bubble_pos_x, bubble_pos_y, 'y');

$\%$ ask if the user would like to continue picking bubbles

$\%$ choose the answer only after any new bubbles in the image have been zoomed $\%$ to

disp('would you like pick bubbles?');

rep=input ('enter 1 for yes or 0 for no: ');

end

\%extract the coordinates of the flare origin

flare_origin_x=bubble_pos_x $(1,1)$;

flare_origin_y=bubble_pos_y $(1,1)$;

\%extact the coordinates of the bubble long and short axes

bubble_pos_x=transpose (bubble_pos_x $(2:$ length (bubble_pos_x $))$ );

bubble_pos_y=transpose (bubble_pos_y $(2:$ length (bubble_pos_y $))$ );

\subsection{Canadian_Grid}

\%constructs a Canadian Grid for the chosen frame

$\%$ run the moduel 'laser_detect' to locate the image coordinates of the left $\%$ and right laser

laser_detect3

$\%$ choose the spacing distance of the Canadian Grid grid_spacing $=20$;

$\%$ specify the spacing of the lasers for the camera that was used laser_spacing_cm $=20$;

\%horizontal and vertical camera angles are corrected for the the diffracton \%of light through water instead of the original values that were calculated $\%$ for air

\%horizontal angle of view beta $=\operatorname{asin}(((68 * \mathrm{pi} / 180) * 1.00028) / 1.334) ; \%$ angle in radians 
$\%$ vertical angle of view

alph=asin (( (55*pi/180)*1.00028)/1.334); \%angle in radians

$\%$ dip angle of camera below horizontal

theta $=45 * \mathrm{pi} / 180 ; \%$ angle in radians

$\%$ calculate the image distance between the lasers

laser_spacing_pix=pt2av(1,1)-pt1av $(1,1)$; \%image length corresponding to $20 \mathrm{~cm}$

$\%$ conversion scale between image and scene for the horizontal line through

$\%$ the laser points

scale_pix_cm=laser_spacing_cm/laser_spacing_pix;

$\%$ calculate image distances

$\operatorname{gp}=\operatorname{length}(\operatorname{imgA}(1,:, 1)) / 2$;

$\mathrm{bp}=\operatorname{length}(\operatorname{imgA}(:, 1,1)) / 2$;

$\%$ calculate the distance from the origin to $\mathrm{p}$

$O p=\tan (a l p h / 2) * b p$;

$\%$ find the vetical average of the laser points (image distance)

vert_las_av $=(\operatorname{pt} 1 \mathrm{av}(1,2)+\operatorname{pt} 2 \mathrm{av}(1,2)) / 2$;

$\%$ vertical distance of lasers from the centre

$\%$ if the lasers are above the centre line the answer will be positive \%adpated from McGovern (2015)

laser_angle=atan $(($ length $(\operatorname{imgA}(:, 1,1)) / 2$-vert_las_av $) / 0 p)$;

$\%$ scene distance along the trace of the lasers

LR=length (imgA $(1,:, 1)) *$ scale_pix_cm;

$\%$ determine the height of the camera

\%adapted from Wakefield (1987)

$\mathrm{ON}=\mathrm{LR} * \sin ($ theta-laser_angle $) /(2 * \tan ($ beta $/ 2))$;

$\%$ distance from camera to the base line

$\mathrm{OB}=\mathrm{ON} / \sin ($ theta $+\mathrm{al} \mathrm{ph} / 2)$;

$\%$ scene distance along the baseline

$\mathrm{EF}=2 * \mathrm{OB} * \tan ($ beta $/ 2)$; 
$\%$ scale along the base line

$\operatorname{Shb}=$ length $(\operatorname{imgA}(1,:, 1)) / E F$;

$\%$ scale along the laser line

Shp=length $(\operatorname{imgA}(1,:, 1)) /$ LR;

$\%$ create points at grid spacing intervals for the vertical scale for BA=grid_spacing:grid_spacing: 1000 ;

$\mathrm{xba}(\mathrm{BA})=\operatorname{bp} *(1+(\tan (\operatorname{theta}-\operatorname{atan}(\mathrm{ON} /(\mathrm{BA}+\mathrm{ON} * \cot (\operatorname{theta}+\mathrm{alph} / 2))))) \ldots$

$/ \tan (\mathrm{alph} / 2))$;

if $\mathrm{xba}(\mathrm{BA})>2 * \mathrm{bp}$

break

end

end

$\%$ take on only the positive values

$\mathrm{xba}=\mathrm{xba}(\mathrm{xba}>0)$;

$\%$ start from bottom (image coordinates are reversed from converntional matlab $\%$ coordinates)

xba=length (imgA $(:, 1,1))-x b a$;

$\%$ calculate y coordinates for horizontal lines

for $i=-2000: 3000$

for $a=1:$ length (xba);

$y b a(i+2001, a)=x b a(1, a)$;

end

end

$i=-2000: 3000$;

$\%$ calculate the intercept along the baselines

for $n=1: 20$

base_distances $(n, 1)=$ Shb*grid_spacing*n;

end

$\%$ plot points outwards from the middle

for $n=1:$ length (base_distances)

base_points_l $(n, 1)=$ length $(\operatorname{imgA}(1,:, 1)) / 2$-base_distances $(n, 1)$;

base_points_r $(n, 1)=$ length $(\operatorname{imgA}(1,:, 1)) / 2+$ base_distances $(n, 1)$;

end 
base_points_l=flipud (base_points_l);

base_points=zeros (length (base_points_l) +length (base_points_r) $+1,2$ ) ;

base_points $(:, 1)=[$ base_points_l; $\operatorname{length}(\operatorname{imgA}(1,:, 1)) / 2$; base_points_r] ;

base_points $(:, 2)=$ length $(\operatorname{imgA}(:, 1,1))$;

\%calculate the intercept along the horizontal line through the laser points for $n=1: 20$

mid_distances $(n, 1)=$ Shp*grid_spacing*n;

end

$\%$ plot the points outwards from the centre

for $\mathrm{n}=1$ : length (mid_distances)

mid_points_l $(\mathrm{n}, 1)=$ length $(\operatorname{imgA}(1,:, 1)) / 2$-mid_distances $(\mathrm{n}, 1)$;

mid_points_r $(\mathrm{n}, 1)=$ length $(\operatorname{imgA}(1,:, 1)) / 2+$ mid_distances $(\mathrm{n}, 1)$;

end

mid_points_l=flipud(mid_points_l);

mid_points=zeros $($ length (mid_points_l) +length (mid_points_r $)+1,2)$;

mid_points $(:, 1)=[$ mid_points_1; length $(i m g A(1,:, 1)) / 2$; mid_points_r] ;

$\% y$ coordinates of all points are the vertical distance to the averaged laser

$\%$ points

mid_points $(:, 2)=$ vert_las_av;

$\%$ plot the lines over the frame image

figure

imshow (imgA);

hold on

plot (i,yba, 'r')

$\operatorname{grads}(:, 1)=($ mid_points $(:, 2)-$ base_points $(:, 2)) . /\left(\operatorname{mid} \_p o i n t s(:, 1)-\right.$ base_points $\left.(:, 1)\right)$;

for $x=-5000: 5000$

for $a=1:$ length (grads)

$y(x+5001, a)=\operatorname{grads}(a, 1) *(x$-base_points $(a, 1))+$ base_points $(a, 2)$;

end

end

$x=-5000: 5000$;

hold on

$\operatorname{plot}\left(\mathrm{x}, \mathrm{y}, \mathrm{\prime}^{\mathrm{r}}\right.$ ')

hold on

line $([\operatorname{length}(\operatorname{imgA}(1,:, 1)) / 2, \operatorname{length}(\operatorname{imgA}(1,:, 1)) / \ldots$ 
2], [0, length (imgA $(:, 1,1))]$, 'color', 'r');

\subsection{Laser_Detect}

$\%$ locates and differentiates the left and right lasers

$\%$ find pixels with a red values higher thatn 0.98 (where each pixel values $\%$ ranges $0-1$ )

$[\mathrm{col}, \mathrm{row}]=\mathrm{f}$ ind $(\operatorname{imgA}(:,:, 1)>=0.98)$;

$\%$ calculate the row differences between each red point and every other red $\%$ point in the frame

for rownum $=1:$ length (row)

distances $(:$, rownum $)=$ row $($ rownum, 1$)-$ row $(:, 1)$;

end

$\%$ set up an array of zeros for inputting the points contained in a cluster differences=zeros $($ length $($ distances $(:, 1)), 1)$;

$\%$ find clusters of red points

for rownum 1 : length (row)

$\%$ take each single column out

diff $=$ distances $(:$, rownum);

$\%$ find the points within 6 pixels of surrounding points

$\%$ if there are less than 12 points in the proximity

if length(diffi) $<5$

$\%$ record the number of that row

differences (rownum, 1) =rownum;

end

$\%$ reset

$\operatorname{diff}=[]$;

$\operatorname{diffi}=[]$;

end

$\%$ take only the located points

differences $=$ differences $($ differences $=0)$; 
$\%$ reject coordinates outside of clusters

for diffnum $1:$ length (differences)

$\operatorname{col}($ differences $($ diffnum $))=$ nan;

row $(\operatorname{differences}(\operatorname{diffnum}))=$ nan;

end

$\operatorname{col}=\operatorname{col}(\operatorname{isnan}(\operatorname{col}))$;

row $=$ row $(i \operatorname{snan}($ row $))$;

\%differentiate points belonging to the left laser (pt1) and right laser (pt2) $\operatorname{pt} 1=[\operatorname{row}(1,1) \operatorname{col}(1,1)]$;

rowdif $=$ row-pt $1(1,1)$;

length (rowdif (rowdif<10))

$\operatorname{pt} 1=[\operatorname{row}(1: \operatorname{length}(\operatorname{rowdif}(\operatorname{rowdif}<10))), \operatorname{col}(1: \operatorname{length}(\operatorname{rowdif}(\operatorname{rowdif}<10)))]$;

pt2 $=[$ row $($ length $($ rowdif $($ rowdif $<10))+\ldots$

$1:$ length (row)), col (length (rowdif (rowdif<10) $+1:$ length $(\operatorname{col}))]$;

\%average the coordinates for each side to obtain 2 distinct points $\operatorname{pt1av}=[\operatorname{mean}(\operatorname{pt} 1(:, 1))$, mean $(\operatorname{pt} 1(:, 2))]$;

pt2av $=[$ mean $(\operatorname{pt} 2(:, 1))$, mean $(\operatorname{pt} 2(:, 2))]$;

$\%$ display the laser points on the image to check that no other red (possily $\%$ from coloured fish) have disrupted the calculations

figure; imshow(imgA); hold on;

scatter (pt1av $(:, 1)$, pt1av (:,2), 'filled');

scatter (pt2av $(:, 1)$, pt2av (:,2), 'filled');

\subsection{Canadian_Grid_Measure}

$\%$ measures the maximum possible size of each bubble using the Canadian Grid

$\%$ create a new array of bubble_pos_x where the 1st,2nd and 3rd,4th multiple $\%$ enties (those belonging to each axis end)

$\%$ are ordered left, right (smallest $[\mathrm{x}]$, largest $[\mathrm{x}]$ )

bubble_pos_ver=[bubble_pos_x, bubble_pos_y] ;

for $n=1:$ length (bubble_pos_x) $/ 4$

$\%$ number of bubbles selected

bubble_pos_ver $(1+4 *(n-1): 2+\ldots$

$4 *(n-1),:)=$ sort (bubble_pos_ver $(1+4 *(n-1): 2+4 *(n-1),:$ ) ) ; 
bubble_pos_ver $(3+4 *(n-1): 4+4 *(n-1),:)=\operatorname{sort}($ bubble_pos_ver $(3+4 *(n-1): 4+4 *(n-1),:))$; end

$\% \mathrm{bk}$ is pixel distance between interscetion points on the baseline bk=abs (base_points $(2,1)$-base_points $(1,1)$ );

$\%$ measure each bubble based on the constucted grid

for $n=1:$ length(bubble_pos_x) $/ 4 \%$ number of bubbles selected

\section{\%MATRIX CONVENTION}

$\%$ dir represents each of the four ordered picks for each bubble

$\% \operatorname{dir}=1:$ axis 1 , left end

$\% \operatorname{dir}=2:$ axis 1 , right end

$\% \operatorname{dir}=3:$ axis 2 , left end

$\% \operatorname{dir}=4:$ axis 2 , right end

for $\operatorname{dir}=1: 4$

$\%$ plot each of the horizontal picks

hold on

scatter $\left(\left[b u b b l e_{-} p o s_{-} x(1+4 *(n-1), 1)\right.\right.$, bubble_pos_x $(2+4 *(n-\ldots$

1), 1), bubble_pos_ver $(3+4 *(n-1), 1)$, bubble_pos_ver $(4+4 *(n-\ldots$

$1), 1)], \quad\left[b u b b l e \_p o s \_y(1+4 *(n-1), 1)\right.$, bubble_pos_y $(2+\ldots$

$4 *(n-1), 1)$, bubble_pos_ver $(3+4 *(n-1), 2)$, bubble_pos_ver $(4+4 *(n-1), 2)])$;

$\%$ calculate the horizontal image distance to each axis edge ac_ax $(n, \operatorname{dir})=a b s($ bubble_pos_ver $(\operatorname{dir}+4 *(n-1), 1)-\operatorname{length}(\operatorname{imgA}(1,:, 1)) / 2)$;

$\%$ ba is the vertical image distance to the $y$ coordinate of each axis point

ba_ax $(n, \operatorname{dir})=\operatorname{abs}($ length $(\operatorname{imgA}(:, 1,1))-$ bubble_pos_ver $(\operatorname{dir}+4 *(n-1), 2))$;

$\%$ find the $\mathrm{x}$ coordinates of all interecepts to the grid, along the line of $\%$ each bubble

c_ax_ints $(:, n, \operatorname{dir})=($ bubble_pos_ver $(\operatorname{dir}+4 *(n-1), 2)-($ base_points $(:, 2)-\ldots$

$\operatorname{grads}(:, 1) . *$ base_points $(:, 1))) . / \operatorname{grads}(:, 1)$;

end

end

for $n=1:$ length (bubble_pos_x) $/ 4$

for $\operatorname{dir}=1: 4$

$\%$ find the intercept that is closest to the $\mathrm{x}$ coordinate of the 
$\%$ centre line

al_ax $(\mathrm{n}, \operatorname{dir})=\min \left(\operatorname{abs}\left(\right.\right.$ length $(\operatorname{imgA}(1,:, 1)) / 2-c_{-}$ax_ints $\left.\left.(:, \mathrm{n}, \mathrm{dir})\right)\right)$;

end

end

$\%$ calculate true distances of horizontal and vertical axis end points

for $\operatorname{dir}=1: 4$

\%eq 13 from wakefield -BK is the laser spacing $(\mathrm{cm})$

$A C_{-} a x(:, \operatorname{dir})=\left(a c_{-} a x(:, \operatorname{dir}) . / a l_{-} a x(:, \operatorname{dir})\right) * l_{a s e r}$ spacing_cm;

BA_ax $(:, \operatorname{dir})=0$ N. / (tan (theta-atan $\left(\left(b_{-} a x(:, \operatorname{dir}) . / b p-1\right) * \ldots\right.$

$\tan (\mathrm{alph} / 2))))-\mathrm{ON} * \cot ($ theta $+\mathrm{al} \mathrm{ph} / 2)$;

end

$\%$ find the differences for the vertical (BA) axes and horizontal (AC) axes

$\% n=1:$ axis 1 differences

$\% \mathrm{n}=2$ : axis 2 differences

for $\mathrm{n}=1: 2$

$\mathrm{AC}_{-} \operatorname{diff}(:, \mathrm{n})=\operatorname{abs}\left(\mathrm{AC}_{-} \mathrm{ax}(\mathrm{n}, 1+(\mathrm{n}-1) * 2)-\mathrm{AC}_{-} \mathrm{ax}(\mathrm{n}, 2+(\mathrm{n}-1) * 2)\right)$;

$B_{-} \operatorname{diff}(:, n)=a b s\left(B A_{-} a x(n, 1+(n-1) * 2)-B_{-} a x(n, 2+(n-1) * 2)\right)$;

end

\% use pythagoras to calculate the lengths of axes 1 \& 2

for $n=1:$ length (bubble_pos_x) $/ 4$

tot_dist_hor $(\mathrm{n}, 1)=\operatorname{sqrt}\left(\left(\mathrm{BA}_{-} \operatorname{diff}(:, 1)\right)^{\wedge} 2+\left(\operatorname{AC} \_\operatorname{diff}(:, 1)\right)^{\wedge} 2\right)$;

tot_dist_ver $(\mathrm{n}, 1)=\operatorname{sqrt}\left(\left(\mathrm{BA}_{-} \operatorname{diff}(:, 2)\right)^{\wedge} 2+\left(\operatorname{AC} \_\operatorname{diff}(:, 2)\right)^{\wedge} 2\right)$;

end

\section{9 bubble_size_and_pos}

$\%$ calculates the true size of bubble radius and elevation above the sea floor

$\%$ calculate the centre points of each bubble by taking the mean of all axes $\%$ end points

for $\mathrm{n}=1:$ length(bubble_pos_x) $/ 4$

$\%$ centred $\mathrm{x}$ and $\mathrm{y}$ coordinate

$\begin{array}{llll}\% & \mathrm{x} 1 & \mathrm{x} 2 & \ldots\end{array} \mathrm{xn}$ 
$\%$ y1 $\mathrm{y} 2 \ldots \mathrm{yn}$

bubble_centre $(:, n$, iteration $)=[($ bubble_pos_x $(1+4 *(n-1), 1)+\ldots$

bubble_pos_x $(2+4 *(n-1), 1)+$ bubble_pos_ver $(3+4 *(n-1), 1)+$ bubble_pos_ver $(4+\ldots$ $4 *(n-1), 1)) / 4$, (bubble_pos_y $(1+4 *(n-1), 1)+$ bubble_pos_y $(2+\ldots$

$4 *(n-1), 1)+$ bubble_pos_ver $(3+4 *(n-1), 2)+$ bubble_pos_ver $(4+4 *(n-1), 2)) / 4]$;

end

$\%$ calculate the image distance from the bottom of the image to each bubble

bw=length (imgA $(:, 1,1))-\ldots$

bubble_centre $(2,1:$ length (bubble_centre $(2,1:$ length (bubble_pos_x) $/ \ldots$

4 ,iteration)), iteration);

$\%$ calculate the image distance from the bottom of the image to the

$\%$ flare origin

bv=length (imgA $(:, 1,1))$-flare_origin_y;

$\%$ calcualte the angle bewteen the line from 'origin' to 'vent'

$\%$ and the vertical

$\mathrm{v}=\mathrm{pi} / 2-($ theta $+\mathrm{alph} / 2)+(\mathrm{bv} /$ length $(\operatorname{imgA}(:, 1,1))) * \mathrm{alph}$;

$\%$ calcualte the angle bewteen the line from 'origin' to 'bubble centre'

$\%$ and the vertical

phi=pi/2-(theta + alph/2) $+($ bw . /length $(i m g A(:, 1,1))) * a l p h$;

\%calculate the elevation of each bubble above the seafloor

$\mathrm{NV}=\mathrm{ON} * \tan (\mathrm{v})$;

$\mathrm{NW}=\mathrm{ON} . * \tan (\mathrm{phi})$;

bubble_height (iteration, $1:$ length (bubble_pos_x $) / 4)=($ NW-NV).$/ \tan ($ phi) ;

$\%$ calulate the distance from the origin to the end of the line through the $\%$ bubble

$\mathrm{OW}=\mathrm{ON} . / \mathrm{cos}(\mathrm{phi})$;

$\%$ calculate the distance from the origin to the centre of the image $\mathrm{Op}=(\mathrm{bp} / 10) / \tan (\mathrm{al} \mathrm{ph} / 2)$;

$\%$ calculate the distance from the origin to the intersection of the along $\% \mathrm{OW}$

$\mathrm{Ow}_{\mathrm{w}}=0 \mathrm{p} \cdot / \cos (\mathrm{phi}-(\mathrm{pi} / 2-$ theta $)) ;$ 
$\%$ run the module 'off_centre' that accounts for bubbles drifting from vertical $\%$ advection paths

off_centre

$\%$ horizontal angle of the bubble

ce_beta=atan $\left(\left(a c_{-} c e / 10\right) / 0 p\right)$;

\%calculate the distance along OW between the bubble and the seafloor WPhi=(bubble_height (iteration, $1:$ length (bubble_pos_x $) / 4) . / \cos ($ phi $)) . / \ldots$ transpose (cos (ce_beta));

$\%$ calculate the length of the line along OW between the image and the seafloor $\mathrm{wW}=(\mathrm{OW}-\mathrm{OW}) . /$ transpose $(\cos ($ ce_beta $))$;

for $n=1:$ length (bubble_pos_x) $/ 4$

$\%$ convert diameters to radii

$\max _{-} a 1(\mathrm{n},:)=$ tot_dist_hor $(\mathrm{n}, 1) / 2$;

$\max \_a 2(n,:)=$ tot_dist_ver $(n, 1) / 2$;

$\%$ find the minimum length by finding the pixel distance and scaling it to $\%$ the laser spacing min_a1 $(n,:)=\left(\left(\operatorname{sqrt}\left(\left(\text { bubble_pos_ver }(2+4 *(n-1), 1)-\operatorname{bubble} \_p o s_{-} \operatorname{ver}(1+4 *(n-1), 1)\right)^{\wedge} 2+\ldots\right.\right.\right.$ (bubble_pos_ver $(2+4 *(n-1), 2)-$ bubble_pos_ver $\left.\left.\left.(1+4 *(n-1), 2))^{\wedge} 2\right)\right) / 2\right) / 10$; min_a2 $(n,:)=((\operatorname{sqrt}(($ bubble_pos_ver $(3+4 *(n-1), 1)-$ bubble_pos_ver $(4+4 *(n-1), 1)) \wedge 2+\ldots$ (bubble_pos_ver $(3+4 *(n-1), 1)-$ bubble_pos_ver $\left.\left.\left.(4+4 *(n-1), 1))^{\wedge} 2\right)\right) / 2\right) / 10$; end

\%work out how far the bubble has strayed $\%$ run the module 'uncertainty' that calculates uncertainties uncertainty

$\%$ calculate the true length of each bubble axis using the equation adapted \% from (McGovern 2012)

for $n=1:$ length (bubble_pos_x) $/ 4$

a1 $(:, \mathrm{n})=\min _{-} \mathrm{a} 1(\mathrm{n},:)+\left(\max _{-} \mathrm{a} 1(\mathrm{n},:)-\min _{-} \mathrm{a} 1(\mathrm{n},:)\right) *(\mathrm{wW}(:, \mathrm{n})-\operatorname{WPhi}(:, \mathrm{n})) / \mathrm{wW}(:, \mathrm{n})$; a2 $(:, \mathrm{n})=\min _{-} a 2(\mathrm{n},:)+\left(\max _{-} a 2(\mathrm{n},:)-\min _{-} \mathrm{a} 2(\mathrm{n},:)\right) *(\mathrm{wW}(:, \mathrm{n})-\mathrm{WPhi}(:, \mathrm{n})) / \mathrm{wW}(:, \mathrm{n})$; end

$\%$ calculate the equivalent sqherical radius for each of the bubbles for $\mathrm{n}=1$ : length (bubble_pos_x) $/ 4$ bubble_radius $($ iteration,$n)=n \operatorname{throot}(a 1(:, n) \wedge 2 * a 2(:, n), 3)$; 
end

$\%$ display the radius

disp('bubble radius ( $\mathrm{cm}$ )')

disp(bubble_radius(iteration, 1 : length(bubble_pos_x)/4));

$\%$ display the bubble height

disp ('bubble height ( $\mathrm{cm}$ )')

disp(bubble_height(iteration, 1 : length(bubble_pos_x)/4));

\subsection{0 off_centre}

\%accounts for how much each bubble has strayed in a horizontal direction

for $\mathrm{n}=1:$ length(bubble_pos_x) $/ 4 \%$ number of bubbles selected

\%horizontal distance to each bubble centre from the image centre

ac_ce $(\mathrm{n},:)=\operatorname{abs}($ bubble_centre $(1, \mathrm{n}$, iteration) $-\operatorname{length}(\operatorname{imgA}(1,:, 1)) / 2)$;

end

\%horizontal distance to the vent

ac_ve=abs (flare_origin_x-length $(\operatorname{imgA}(1,:, 1)) / 2)$;

$\%$ find the $\mathrm{x}$ coordinates of all interecepts along the line of the vent

c_ve_ints $=(f$ lare_origin_x-(base_points $(:, 2)-\operatorname{grads}(:, 1) . * \ldots$

base_points $(:, 1)))$. /grads $(:, 1)$;

$\%$ find the intercept that is closest to the $\mathrm{x}$ coordinate of the centre line

al_ve=min $\left(\operatorname{abs}\left(\right.\right.$ length $(\operatorname{imgA}(1,:, 1)) / 2-c_{-}$ve_ints $\left.)\right)$;

$\%$ calculate the true horizontal distance to the vent

AC_ve $=($ ac_ve/al_ve $) *$ laser_spacing_cm;

$\%$ calculate the true horizontal distance to the bubble centre

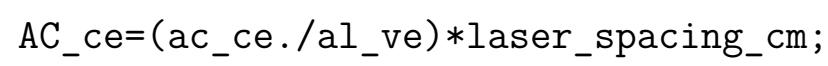

$\%$ calculate the true horizontal differnce between the vent and each bubble $\%$ centre

offvent_true_dist=abs (AC_ve-AC_ce);

$\%$ calculate the horizontal image differnce between the vent and each bubble $\%$ centre

orig_ce_diff_pix=abs (ac_ve-ac_ce); 


\subsection{1 uncertainty}

$\%$ calculates errors based on how far the bubble is likely to have strayed \%away from vertical advection

$\%$ horizontal angle of the vent

ve_beta=atan ( (ac_ve/10)/Op);

\%horizontal distance alonh OW that the bubble is likely to have strayed towards $\%$ and away-from the camera

$S(1,:)=o f f v e n t \_t r u e \_d i s t . / \cos \left(c_{-}\right.$beta $)$;

$\%$ calculate the +/- uncertainty off the height values

$\%$ (delta_phi) in Figure 2.12

vert_unc $=\mathrm{S} . * \tan (\mathrm{pi} / 2-\mathrm{psi})$;

$\%$ calculate the +/- that the bubble could be displaced along the line wW $\%$ either side of the zenith to the vent

hypoff=S./cos (pi/2-psi);

$\%$ calculate maximum and minumum uncertainty bubble sizes

a1_min_unc=min_a1+(max_a1-min_a1). *transpose $((w W-(W P S I+h y p o f f)) . / w W)$;

a2_min_unc=min_a2+(max_a2-min_a2) . *transpose ( (wW- (WPSI+hypoff $))$. /wW) ;

a1_max_unc=min_a1+(max_a1-min_a1). *transpose $((w W-(W P S I-h y p o f f)) . / w W)$;

a2_max_unc=min_a2+(max_a2-min_a2) . *transpose $(($ wW- $($ WPSI-hypoff $))$./wW) ;

$\%$ set up empty arrays for maximum and minimum uncertainties

bubble_radius_max_unc $=$ zeros $(100,100)$;

bubble_radius_min_unc $=$ zeros $(0,100)$;

$\%$ calculate max and min bubble axes sizes and populate the empty arrays

for $\mathrm{n}=1$ : length(bubble_pos_x) $/ 4$

bubble_radius_max_unc (iteration, $\mathrm{n})=\operatorname{nthroot}(a 1$ max_unc $(\mathrm{n},:) . \wedge 2 . * a 2$ max_unc $(\mathrm{n},:), 3)$;

bubble_radius_min_unc (iteration, $\mathrm{n})=\operatorname{nthroot}\left(\mathrm{a} 1\right.$ _min_unc $(\mathrm{n},:) .{ }^{\wedge} 2 . * a 2$ min_unc $\left.(\mathrm{n},:), 3\right)$; end 
Appendix

\%add error for sinusoidal variations

bubble_radius_max_unc=bubble_radius_max_unc+0.0001;

bubble_radius_min_unc=bubble_radius_min_unc +0.0001 ; 


\section{Bibliography}

Allison, P. A., Hesselbo, S. P., and Brett, C. E. (2008). Methane seeps on an early Jurassic dysoxic seafloor. Palaeogeography, Palaeoclimatology, Palaeoecology, 270(3):230238.

Anis, A. and Moum, J. (1992). The superadiabatic surface layer of the ocean during convection. Journal of Physical Oceanography, 22(10):1221-1227.

Bangs, N. L., Westbrook, G. K., Ladd, J. W., and Buhl, P. (1990). Seismic velocities from the Barbados Ridge Complex: Indicators of high pore fluid pressures in an accretionary complex. Journal of Geophysical Research: Solid Earth (1978-2012), 95(B6):8767-8782.

Barker, D. H., Sutherland, R., Henrys, S., and Bannister, S. (2009). Geometry of the Hikurangi subduction thrust and upper plate, North Island, New Zealand. Geochemistry, Geophysics, Geosystems, 10(2).

Barnes, P. M., Lamarche, G., Bialas, J., Henrys, S., Pecher, I., Netzeband, G. L., Greinert, J., Mountjoy, J. J., Pedley, K., and Crutchley, G. (2010). Tectonic and geological framework for gas hydrates and cold seeps on the Hikurangi Subduction Margin, New Zealand. Marine Geology, 272(1):26-48.

Barnes, P. M., Nicol, A., and Harrison, T. (2002). Late Cenozoic evolution and earthquake potential of an active listric thrust complex above the Hikurangi Subduction Zone, New Zealand. Geological Society of America Bulletin, 114(11):1379-1405.

Barr, R. and Coombs, R. F. (2005). The significance of high-order resonances of spherical bubbles to the acoustic response of fish with swimbladders. The Journal of the Acoustical Society of America, 117(6):3589-3599.

Bashkatov, A. N. and Genina, E. A. (2003). Water refractive index in dependence on temperature and wavelength: a simple approximation. In Saratov Fall Meeting 2002: Optical Technologies in Biophysics and Medicine IV, pages 393-395. International Society for Optics and Photonics.

Berndt, C., Feseker, T., Treude, T., Krastel, S., Liebetrau, V., Niemann, H., Bertics, V. J., Dumke, I., Dünnbier, K., Ferré, B., et al. (2014). Temporal constraints on hydrate-controlled methane seepage off Svalbard. Science, 343(6168):284-287. 
Bhaga, D. and Weber, M. (1981). Bubbles in viscous liquids: shapes, wakes and velocities. Journal of Fluid Mechanics, 105:61-85.

Bohrmann, G., Greinert, J., Suess, E., and Torres, M. (1998). Authigenic carbonates from the Cascadia subduction zone and their relation to gas hydrate stability. Geology, 26(7):647-650.

Buffett, B. and Archer, D. (2004). Global inventory of methane clathrate: sensitivity to changes in the deep ocean. Earth and Planetary Science Letters, 227(3):185-199.

Campbell, K. A., Francis, D. A., Collins, M., Gregory, M. R., Nelson, C. S., Greinert, J., and Aharon, P. (2008). Hydrocarbon seep-carbonates of a miocene forearc (east coast basin), north island, new zealand. Sedimentary Geology, 204(3):83-105.

Cathles, L., Su, Z., and Chen, D. (2010). The physics of gas chimney and pockmark formation, with implications for assessment of seafloor hazards and gas sequestration. Marine and Petroleum Geology, 27(1):82-91.

Chadwick, W. W., Merle, S. G., Buck, N. J., Lavelle, J. W., Resing, J. A., and Ferrini, V. (2014). Imaging of $\mathrm{CO}_{2}$ bubble plumes above an erupting submarine volcano, NW Rota-1, Mariana Arc. Geochemistry, Geophysics, Geosystems, 15(11):4325-4342.

Clark, J. F., Washburn, L., Hornafius, J. S., and Luyendyk, B. P. (2000). Dissolved hydrocarbon flux from natural marine seeps to the southern California Bight. Journal of Geophysical Research: Oceans (1978-2012), 105(C5):11509-11522.

Clarke, J. H. (2006). Applications of multibeam water column imaging for hydrographic survey. Hydrographic Journal, 120:3.

Clay, C. S. and Medwin, H. (1977). Acoustical oceanography: principles and applications.

Collot, J.-Y., Delteil, J., Lewis, K. B., Davy, B., Lamarche, G., Audru, J.-C., Barnes, P., Chanier, F., Chaumillon, E., Lallemand, S., et al. (1996). From oblique subduction to intra-continental transpression: structures of the southern KermadecHikurangi margin from multibeam bathymetry, side-scan sonar and seismic reflection. Marine Geophysical Researches, 18(2-4):357-381.

Craig, P. D. and Banner, M. L. (1994). Modeling wave-enhanced turbulence in the ocean surface layer. Journal of Physical Oceanography, 24(12):2546-2559.

Crutchley, G. J., Pecher, I. A., Gorman, A. R., Henrys, S. A., and Greinert, J. (2010). Seismic imaging of gas conduits beneath seafloor seep sites in a shallow marine gas hydrate province, Hikurangi Margin, New Zealand. Marine Geology, 272(1):114-126. 
Dando, P. R., O'Hara, S. C., Schuster, U., Taylor, L. J., Clayton, C. J., Baylis, S., and Laier, T. (1994). Gas seepage from a carbonate-cemented sandstone reef on the Kattegat coast of Denmark. Marine and Petroleum Geology, 11(2):182-189.

Datta, R., Napier, D., and Newitt, D. (1950). The properties and behaviour of gas bubbles formed at circular orifices. In CHEMISTRY \& INDUSTRY, number 9, pages 168-168. SOC CHEMICAL INDUSTRY 14 BELGRAVE SQUARE, LONDON SW1X 8PS, ENGLAND.

Davey, F., Hampton, M., Childs, J., Fisher, M., Lewis, K., and Pettinga, J. (1986a). Structure of a growing accretionary prism, Hikurangi Margin, New Zealand. Geology, 14(8):663-666.

Davey, F., Lewis, K., Childs, J., and Hampton, M. (1986b). Section 3: Displays of seismic sections: Convergent Margin off East Coast of North Island, New Zealand, parts i and ii.

Davis, D., Suppe, J., and Dahlen, F. (1983). Mechanics of fold-and-thrust belts and accretionary wedges. J. geophys. Res, 88(B2):1153-1172.

Dimitrov, L. I. (2002). Mud volcanoes - the most important pathway for degassing deeply buried sediments. Earth-Science Reviews, 59(1):49-76.

Din, F. (1961). Thermodynamic Functions of Gases: Methane, nitrogen, ethane, volume 3. Butterworths Scientific Publications.

Drennan, W., Kahma, K., Terray, E., Donelan, M., and Kitaigorodskii, S. (1992). Observations of the enhancement of kinetic energy dissipation beneath breaking wind waves. In Breaking waves, pages 95-101. Springer.

Duda, J. and Vrentas, J. (1969). Mathematical analysis of bubble dissolution. AIChE Journal, 15(3):351-356.

Dupré, S., Scalabrin, C., Grall, C., Augustin, J.-M., Henry, P., Şengör, A., Görür, N., Çă̆atay, M. N., and Géli, L. (2015). Tectonic and sedimentary controls on widespread gas emissions in the Sea of Marmara: Results from systematic, shipborne multibeam echo sounder water column imaging. Journal of Geophysical Research: Solid Earth.

Ehhalt, D. and Schmidt, U. (1978). Sources and sinks of atmospheric methane. Pure and Applied Geophysics, 116(2-3):452-464.

Ellingsen, K. and Risso, F. (2001). On the rise of an ellipsoidal bubble in water: oscillatory paths and liquid-induced velocity. Journal of Fluid Mechanics, 440:235268. 
Faure, K., Greinert, J., von Deimling, J. S., McGinnis, D. F., Kipfer, R., and Linke, P. (2010). Methane seepage along the Hikurangi Margin of New Zealand: Geochemical and physical data from the water column, sea surface and atmosphere. Marine Geology, 272(1):170-188.

Fdhila, R. B. and Duineveld, P. (1996). The effect of surfactant on the rise of a spherical bubble at high Reynolds and Peclet numbers. Physics of Fluids (1994present), 8(2):310-321.

Field, B. D. and Uruski, C. (1997). Cretaceous-Cenozoic geology and petroleum systems of the East Coast region, New Zealand, volume 1. Institute of Geological \& Nuclear Sciences.

Forsberg, F., Shi, W., and Goldberg, B. (2000). Subharmonic imaging of contrast agents. Ultrasonics, 38(1):93-98.

Gargett, A. E. (1989). Ocean turbulence. Annual Review of Fluid Mechanics, 21(1):419-451.

Gibson, R., Atkinson, R., and Gordon, J. (2005). Ecology of cold seep sediments: interactions of fauna with flow, chemistry and microbes. Oceanography and Marine Biology: an annual review, 43:1-46.

Greinert, J., Bohrmann, G., and Suess, E. (2001). Methane-venting and gas hydraterelated carbonates at the Hydrate Ridge: their classification, distribution and origin.

Greinert, J., Lewis, K., Bialas, J., Pecher, I. A., Rowden, A., Bowden, D., De Batist, M., and Linke, P. (2010). Methane seepage along the Hikurangi Margin, New Zealand: Overview of studies in 2006 and 2007 and new evidence from visual, bathymetric and hydroacoustic investigations. Marine Geology, 272(1):6-25.

Greinert, J. and Nützel, B. (2004). Hydroacoustic experiments to establish a method for the determination of methane bubble fluxes at cold seeps. Geo-Marine Letters, $24(2): 75-85$.

Guliyev, I., Feizullayev, A., Jevanshir, R. D., and Akademiiias (1996). All about mud volcanoes. Geology Institute, Azerbaijan Academy of Sciences.

Hanwright, J., Zhou, J., Evans, G. M., and Galvin, K. P. (2005). Influence of surfactant on gas bubble stability. Langmuir, 21(11):4912-4920.

Hasiotis, T., Papatheodorou, G., Kastanos, N., and Ferentinos, G. (1996). A pockmark field in the Patras Gulf (Greece) and its activation during the 14/7/93 seismic event. Marine Geology, 130(3):333-344. 
Henrys, S., Reyners, M., Pecher, I., Bannister, S., Nishimura, Y., and Maslen, G. (2006). Kinking of the subducting slab by escalator normal faulting beneath the North Island of New Zealand. Geology, 34(9):777-780.

Hornafius, J. S., Quigley, D., and Luyendyk, B. P. (1999). The world's most spectacular marine hydrocarbon seeps (Coal Oil Point, Santa Barbara Channel, California): Quantification of emissions. Journal of Geophysical Research: Oceans (1978-2012), 104(C9):20703-20711.

Hovland, M., Judd, A. G., and Burke, R. (1993). The global flux of methane from shallow submarine sediments. Chemosphere, 26(1):559-578.

Judd, A. (2000). Geological sources of methane. In Atmospheric Methane, pages 280-303. Springer.

Judd, A., Davies, G., Wilson, J., Holmes, R., Baron, G., and Bryden, I. (1997). Contributions to atmospheric methane by natural seepages on the UK continental shelf:[mar. geol. 137 (1997) 165-189] 2pii of original article: S0025-3227 (96) 00087-4.2. Marine Geology, 140(3):427-455.

Judd, A. and Hovland, M. (1992). The evidence of shallow gas in marine sediments. Continental Shelf Research, 12(10):1081-1095.

Judd, A. and Hovland, M. (2007). Seabed fluid flow: the impact on geology, biology and the marine environment. Cambridge University Press.

Judd, A., Hovland, M., Dimitrov, L., Garcia Gil, S., and Jukes, V. (2002). The geological methane budget at continental margins and its influence on climate change. Geofluids, 2(2):109-126.

Judd, A. G. (2004). Natural seabed gas seeps as sources of atmospheric methane. Environmental Geology, 46(8):988-996.

King, L. H. and MacLean, B. (1970). Pockmarks on the Scotian shelf. Geological Society of America Bulletin, 81(10):3141-3148.

Klaucke, I., Weinrebe, W., Petersen, C. J., and Bowden, D. (2010). Temporal variability of gas seeps offshore New Zealand: Multi-frequency geoacoustic imaging of the Wairarapa area, Hikurangi Margin. Marine Geology, 272(1):49-58.

Kvenvolden, K. A., Lorenson, T. D., and Reeburgh, W. S. (2001). Attention turns to naturally occurring methane seepage. Eos, Transactions American Geophysical Union, 82(40):457-457. 
Kvenvolden, K. A. and Pettinga, J. R. (1989). Hydrocarbon gas seeps of the convergent Hikurangi Margin, North Island, New Zealand. Marine and petroleum geology, $6(1): 2-8$.

Lacroix, A. V. (1993). Unaccounted-for sources of fossil and isotopically-enriched methane and their contribution to the emissions inventory: A review and synthesis. Chemosphere, 26(1):507-557.

Lashof, D. A. and Ahuja, D. R. (1990). Relative contributions of greenhouse gas emissions to global warming.

Lee, M. W. and Collett, T. S. (2001). Elastic properties of gas hydrate-bearing sediments. Geophysics, 66(3):763-771.

Leifer, I. and Culling, D. (2010). Formation of seep bubble plumes in the Coal Oil Point seep field. Geo-Marine Letters, 30(3-4):339-353.

Leifer, I. and MacDonald, I. (2003). Dynamics of the gas flux from shallow gas hydrate deposits: interaction between oily hydrate bubbles and the oceanic environment. Earth and Planetary Science Letters, 210(3):411-424.

Leifer, I. and Patro, R. K. (2002). The bubble mechanism for methane transport from the shallow sea bed to the surface: A review and sensitivity study. Continental Shelf Research, 22(16):2409-2428.

Leifer, I., Patro, R. K., and Bowyer, P. (2000). A study on the temperature variation of rise velocity for large clean bubbles. Journal of Atmospheric and Oceanic Technology, 17(10):1392-1402.

León, R., Somoza, L., Medialdea, T., González, F., Díaz-del Río, V., Fernández-Puga, M., Maestro, A., and Mata, M. (2007). Sea-floor features related to hydrocarbon seeps in deepwater carbonate-mud mounds of the Gulf of Cádiz: from mud flows to carbonate precipitates. Geo-Marine Letters, 27(2-4):237-247.

Lewis, K. and Pettinga, J. (1993). The emerging, imbricate frontal wedge of the Hikurangi Margin. Sedimentary basins of the world, 2:225-250.

Lewis, K. B. and Marshall, B. A. (1996). Seep faunas and other indicators of methanerich dewatering on New Zealand convergent margins. New Zealand Journal of Geology and Geophysics, 39(2):181-200.

Liebetrau, V., Eisenhauer, A., and Linke, P. (2010). Cold seep carbonates and associated cold-water corals at the Hikurangi Margin, New Zealand: new insights into fluid pathways, growth structures and geochronology. Marine Geology, 272(1):307-318. 
MacDonald (2002). Transfer of hydrocarbons from natural seeps to the water column and atmosphere. Geofluids, 2(2):95-107.

Mau, S., Valentine, D. L., Clark, J. F., Reed, J., Camilli, R., and Washburn, L. (2007). Dissolved methane distributions and air-sea flux in the plume of a massive seep field, Coal Oil Point, California. Geophysical Research Letters, 34(22).

McGinnis, D. F., Greinert, J., Artemov, Y., Beaubien, S., and Wüest, A. (2006). Fate of rising methane bubbles in stratified waters: How much methane reaches the atmosphere? Journal of Geophysical Research: Oceans (1978-2012), 111(C9).

Medwin, H. and Clay, C. (1998). Fundamentals of Acoustical Oceanography (Academic, San Diego).

Mendelson, H. D. (1967). The prediction of bubble terminal velocities from wave theory. AIChE Journal, 13(2):250-253.

Merlivat, L. and Memery, L. (1983). Gas exchange across an air-water interface: Experimental results and modeling of bubble contribution to transfer. Journal of Geophysical Research: Oceans (1978-2012), 88(C1):707-724.

Miyagi-Kôgakuhakusi, O. (1927). The motion of an air bubble in rising water. Tohoku Imp. Univ. Tech. Reports, 6:135-171.

Miyake, Y. and Koizumi, M. (1948). The measurement of the viscosity coefficient of sea water. J. mar. Res, $7(2): 63$.

Moore, J. C. and Vrolijk, P. (1992). Fluids in accretionary prisms. Reviews of Geophysics, 30(2):113-135.

Mountjoy, J. (2015a). Tan1505 voyage proposal.

Mountjoy, J. (2015b). Tan1508 voyage report.

Muyakshin, S. and Sauter, E. (2010). The hydroacoustic method for the quantification of the gas flux from a submersed bubble plume. Oceanology, 50(6):995-1001.

Nakamura, K., Kawagucci, S., Kitada, K., Kumagai, H., Takai, K., and Okino, K. (2015). Water column imaging with multibeam echo-sounding in the mid-Okinawa Trough: Implications for distribution of deep-sea hydrothermal vent sites and the cause of acoustic water column anomaly. Geochemical Journal, 49(6):579-596.

Newhouse, V. and Shankar, P. M. (1984). Bubble size measurements using the nonlinear mixing of two frequencies. The Journal of the Acoustical Society of America, 75(5):1473-1477. 
Ostrovsky, I. (2003). Methane bubbles in lake kinneret: Quantification and temporal and spatial heterogeneity. Limnology and oceanography, 48(3):1030-1036.

Pecher, I., Henrys, S., Ellis, S., Chiswell, S., and Kukowski, N. (2005). Erosion of the seafloor at the top of the gas hydrate stability zone on the Hikurangi Margin, New Zealand. Geophysical Research Letters, 32(24).

Prasad, R., Mani, N., and Venart, J. (1984). Thermal conductivity of methane. International journal of thermophysics, 5(3):265-279.

Ramaswamy, V., Boucher, O., Haigh, J., Hauglustine, D., Haywood, J., Myhre, G., Nakajima, T., Shi, G., and Solomon, S. (2001). Radiative forcing of climate. Climate change, pages 349-416.

Rehder, G., Leifer, I., Brewer, P. G., Friederich, G., and Peltzer, E. T. (2009). Controls on methane bubble dissolution inside and outside the hydrate stability field from open ocean field experiments and numerical modeling. Marine Chemistry, 114(1):19-30.

Römer, M., Sahling, H., Spieß, V., and Bohrmann, G. (2011). The role of gas bubble emissions at deep-water cold seep systems: An example from the makran continental margin, offshore pakistan. In Proceedings of the 7th International Conference on Gas Hydrates (ICGH 2011), Edinburgh, Scotland, United Kingdom.

Rosenlof, K., Oltmans, S., Kley, D., Russell, J., Chiou, E.-W., Chu, W., Johnson, D., Kelly, K., Michelsen, H., Nedoluha, G., et al. (2001). Stratospheric water vapor increases over the past half-century. Geophysical research letters, 28(7):1195-1198.

Rueff, R. M., Dendy Sloan, E., and Yesavage, V. F. (1988). Heat capacity and heat of dissociation of methane hydrates. AIChE journal, 34(9):1468-1476.

Sahling, H., Bohrmann, G., Artemov, Y. G., Bahr, A., Brüning, M., Klapp, S. A., Klaucke, I., Kozlova, E., Nikolovska, A., Pape, T., et al. (2009). Vodyanitskii mud volcano, Sorokin trough, Black Sea: Geological characterization and quantification of gas bubble streams. Marine and Petroleum Geology, 26(9):1799-1811.

Schmale, O., Greinert, J., and Rehder, G. (2005). Methane emission from high-intensity marine gas seeps in the Black Sea into the atmosphere. Geophysical Research Letters, $32(7)$.

Schneider von Deimling, J., Brockhoff, J., and Greinert, J. (2007). Flare imaging with multibeam systems: data processing for bubble detection at seeps. Geochemistry, Geophysics, Geosystems, 8(6). 
Shakhova, N., Semiletov, I., Leifer, I., Salyuk, A., Rekant, P., and Kosmach, D. (2010). Geochemical and geophysical evidence of methane release over the East Siberian Arctic Shelf. Journal of Geophysical Research: Oceans (1978-2012), 115(C8).

Shankar, P., Chapelon, J., and Newhouse, V. (1986). Fluid pressure measurement using bubbles insonified by two frequencies. Ultrasonics, 24(6):333-336.

Sibuet, M., Juniper, K. S., and Pautot, G. (1988). Cold-seep benthic communities in the Japan subduction zones: geological control of community development. Journal of Marine Research, 46(2):333-348.

Steudler, P., Bowden, R., Melillo, J., and Aber, J. (1989). Influence of nitrogen fertilization on methane uptake in temperate forest soils.

Suess, E. (2010). Marine cold seeps. In Handbook of hydrocarbon and lipid microbiology, pages 185-203. Springer.

Takagi, S. and Matsumoto, Y. (2011). Surfactant effects on bubble motion and bubbly flows. Annual Review of Fluid Mechanics, 43:615-636.

Thuraisingham, R. (1997). New expressions of acoustic cross-sections of a single bubble in the monopole bubble theory. Ultrasonics, 35(5):407-409.

Townend, J. (1997). Subducting a sponge: minimum estimates of the fluid budget of the Hikurangi Margin accretionary prism. Geol. Soc. NZ Newsl, 112(14-16).

Trotsyuk, V. Y. and Avilov, V. (1988). Disseminated flux of hydrocarbon gases from the sea bottom and a method of measuring it. In Doklady Earth Sci, volume 291, pages 218-220.

Tryon, M., Brown, K., and Torres, M. (2002). Fluid and chemical flux in and out of sediments hosting methane hydrate deposits on Hydrate Ridge, or, ii: Hydrological processes. Earth and Planetary Science Letters, 201(3):541-557.

Urick, R. J. (1967). Principles of underwater sound for engineers. Tata McGraw-Hill Education.

Veloso, M., Greinert, J., Mienert, J., and De Batist, M. (2015). A new methodology for quantifying bubble flow rates in deep water using splitbeam echosounders: Examples from the Arctic offshore NW-Svalbard. Limnology and Oceanography: Methods.

Vrolijk, P. (1990). On the mechanical role of smectite in subduction zones. Geology, 18(8):703-707. 
Wakefield, W. W. and Genin, A. (1987). The use of a Canadian (perspective) grid in deep-sea photography. Deep Sea Research Part A. Oceanographic Research Papers, 34(3):469-478.

Wallace, L. M., Reyners, M., Cochran, U., Bannister, S., Barnes, P. M., Berryman, K., Downes, G., Eberhart-Phillips, D., Fagereng, A., Ellis, S., et al. (2009). Characterizing the seismogenic zone of a major plate boundary subduction thrust: Hikurangi Margin, New Zealand. Geochemistry, Geophysics, Geosystems, 10(10).

Woolf, D. K. (1993). Bubbles and the air-sea transfer velocity of gases. AtmosphereOcean, 31(4):517-540.

Woolf, D. K. and Thorpe, S. (1991). Bubbles and the air-sea exchange of gases in near-saturation conditions. Journal of Marine Research, 49(3):435-466. 